\title{
NSW
}

\section{New South Wales Mothers and Babies 2000}


Copyright $@$ NSW Department of Health, November 2001

This work is copyright. It may be reproduced in whole or in part, subject to the inclusion of an acknowledgement of the source and no commercial usage or sale.

State Health Publication No: (PH) 010201

ISBN: 0734733666

Suggested citation:

Public Health Division. New South Wales Mothers and Babies 2000. Sydney: NSW Department of Health, 2001.

Produced by:

Epidemiology and Surveillance Branch

Public Health Division

NSW Department of Health

Locked Bag 961

North Sydney 2059

PH (02) 93919676

FX: (02) 93919232

Further copies of this publication are available from the Public Health Division Web site at: www.health.nsw.gov.au/public-health/mdc/mdcrep00.html. 


\section{TABLE OF CONTENTS}

\section{New South Wales Mothers and Babies 2000}

Introduction

Summary

Data sources

Definitions

Explanatory notes

Method for calculation of risk-adjusted caesarean section rates

Acknowledgements

Further information

Part 1: Trends in New South Wales

1.1 Confinements and births by plurality

Table 1: $\quad$ Births and confinements by plurality, NSW 1996-2000 18

1.2 Health area of residence

Table 2: $\quad$ Confinements by health area of residence, NSW 1996-2000 18

1.3 Maternal age

Figure 1: Confinements among mothers aged less than 20 years and 35 years and over, NSW 1996-2000

Table 3: Confinements by maternal age, NSW 1996-2000

1.4 Maternal country of birth

Table 4: Confinements by maternal country of birth, NSW 1996-2000

1.5 Maternal Aboriginality

Table 5: Confinements by maternal Aboriginality, NSW 1996-2000

1.6 Number of previous pregnancies

Table 6: Confinements by number of previous pregnancies, NSW 1996-2000

1.7 Duration of pregnancy at first antenatal visit

Table 7: Confinements by duration of pregnancy at first antenatal visit, NSW 1996-2000

1.8 Smoking in pregnancy

Table 8: $\quad$ Mothers who smoked at all during pregnancy by number of cigarettes smoked in the second half of pregnancy, NSW 1996-2000 22

1.9 Place of birth

Table 9: $\quad$ Confinements by place of birth, NSW 1996-2000

Figure 2: Confinements by planned place of birth, NSW 1996-2000

1.10 Labour and delivery

Table 10: Confinements by onset and augmentation of labour, NSW 1996-2000 24

Table 11: Confinements by type of delivery, NSW 1996-2000

Table 12: Confinements by health insurance status and type of delivery, NSW 1995-1999

1.11 Pain relief

Table 13: Confinements by type of pain relief, NSW 1998-2000 25

1.12 Baby sex

1.13 Gestational age

Table 14: Births by gestational age, NSW 1996-2000 26

1.14 Birthweight

Table 15: Births by birthweight, NSW 1996-2000

1.15 Apgar score

Table 16: Births by apgar score at five minutes, NSW 1996-2000

1.16 Perinatal outcomes

Table 17: Births by perinatal outcome, NSW 1996-2000 27

1.17 Maternal deaths

Table 18: Maternal deaths by year, NSW 1990-2000

Table 19: Maternal deaths by cause, NSW 1998 


\section{Part 2: Area Health Services}

2.1 Confinements

2.2 Maternal age

2.3 Maternal country of birth

2.4 Maternal Aboriginality

2.5 Place of birth

2.6 Labour and delivery

Table 20: Confinements by maternal age and health area of residence, NSW 2000

Table 21: Confinements by maternal country of birth and health area of residence, NSW 2000

Table 22: Confinements by maternal Aboriginality and health area of residence, NSW 2000

Table 23: Confinements by place of birth and health area of residence, NSW 2000

Table 24: Confinements by onset and augmentation of labour and health area of residence, NSW 2000

Table 25: Confinements by type of delivery and health area of residence, NSW 2000

2.7 Birthweight

Table 26: Births by birthweight and health area of residence, NSW 2000

2.8 Gestational age

Table 27: Births by gestational age and health area of residence, NSW 2000

2.9 Perinatal outcomes

Table 28: Perinatal outcomes by health area of residence, NSW 2000

\section{Part 3: Aboriginal and Torres Strait Islander Mothers and Babies}

3.1 Reporting of Aboriginality

Table 29: Births to Aboriginal and Torres Strait Islander mothers by source

of birth report, year of birth and urban-rural health area of hospital, NSW 1994-1999

Figure 3: Births to Aboriginal and Torres Strait Islander mothers by year of birth and urban-rural health area of hospital, NSW 1994-1999

3.2 Trends in births

Table 30: Aboriginal and Torres Strait Islander mothers and babies by plurality, NSW 1996-2000

3.3 Previous pregnancies

Table 31: Number of previous pregnancies among Aboriginal and Torres Strait Islander mothers, NSW 1996-2000

3.4 Maternal age

Table 32: Age of Aboriginal and Torres Strait Islander mothers, NSW 1996-2000

3.5 Health area of residence

Table 33: Health area of residence of Aboriginal and Torres Strait Islander mothers, NSW 1996-2000

Table 34: Health area of residence of Aboriginal and Torres Strait Islander mothers by age, NSW 2000

3.6 Booking status

3.7 Duration of pregnancy at first antenatal visit

Table 35: Duration of pregnancy at first antenatal visit among Aboriginal and Torres Strait Islander mothers by health area of residence, NSW 2000

3.8 Smoking in pregnancy

Figure 4: Smoking in the second half of pregnancy among Aboriginal and Torres Strait Islander mothers by amount smoked and health area of residence, NSW 2000 
3.9 Medical conditions and obstetric complications

Table 36: Maternal medical conditions and obstetric complications by Aboriginality, NSW 2000

3.10 Labour and delivery

Table 37: Labour onset for Aboriginal and Torres Strait Islander mothers, NSW 1996-2000

Table 38: Type of delivery among Aboriginal and Torres Strait Islander mothers, NSW 1996-2000

3.11 Birthweight

Table 39: Weight of Aboriginal and Torres Strait Islander babies, NSW 1996-2000

Table 40: Weight of Aboriginal and Torres Strait Islander babies by health area of residence, NSW 2000

3.12 Gestational age

Table 41: Gestational age of Aboriginal and Torres Strait Islander babies, NSW 1996-2000

Table 42: Gestational age of Aboriginal and Torres Strait Islander babies by health area of residence, NSW 2000

\subsection{Apgar score}

Table 43: Apgar score of Aboriginal and Torres Strait Islander babies, NSW 1996-2000 44

3.14 Perinatal mortality

Table 44: Perinatal deaths among Aboriginal and Torres Strait Islander babies, NSW 1996-2000

\section{Part 4: Maternal Country of Birth}

4.1 Trends in confinements

Table 45: Confinements and births by country of birth group, NSW 1996-2000

4.2 Maternal age

Table 46: Age of mother by country of birth group, NSW 2000

Figure 5: Age of mother by country of birth group, NSW 2000

4.3 Health Area of residence

4.4 Booking status

4.5 Duration of pregnancy at first antenatal visit

Table 48: Confinements by country of birth group and duration of pregnancy at first antenatal visit, NSW 2000

4.6 Smoking in pregnancy

Table 49: Confinements by country of birth group and smoking in pregnancy, NSW 200048

Table 50: Mothers who smoked at all during pregnancy by number of cigarettes smoked in the second half of pregnancy and country of birth group, NSW 2000

4.7 Medical conditions and obstetric complications

Table 51: Maternal medical conditions and obstetric complications by country of birth group, NSW 2000

4.8 Labour and delivery

Table 52: Labour onset by country of birth group, NSW 200050

Table 53: Type of delivery by country of birth group, NSW 200050

4.9 Birthweight

Table 54: Birthweight by maternal country of birth group, NSW 2000

4.10 Gestational age

Table 55: Gestational age by maternal country of birth group, NSW 2000

4.11 Apgar score $\quad 52$

Table 56: Births by country of birth group and Apgar score at five minutes, NSW 200052

4.12 Perinatal outcomes

Table 57: Perinatal outcomes by country of birth group, NSW 2000 
5.1 Registration rate 53

Table 58: NICUS registrations by health area of residence, NSW \& ACT 2000

Table 59: Confinements by health area of residence and Aborginality, NSW \& ACT 2000

5.2 Maternal characteristics

Table 60: Confinements by health area of residence and maternal age, NSW \& ACT 2000

Figure 6: Confinements by antenatal corticosteroid administration and gestational age, NSW \& ACT 1996-2000

Table 61: Confinements by antenatal corticosteroid administration and gestational age, NSW \& ACT 1996-2000

5.3 Transfer status, labour and delivery

Figure 7: NICUS registrants by place of birth (level of obstetric hospital) and gestational age, NSW and ACT 2000

Table 62: Births by booking status, transfer status and gestational age, NSW \& ACT 2000

Table 63: Births by place of birth (level of obstetric hospital) and gestational age, NSW \& ACT 2000

Table 64: Births by booking status, transfer status and birthweight, NSW \& ACT 2000

Table 65: Births by place of birth (level of obstetric hospital) and birthweight,

NSW \& ACT 2000

Table 66: Confinements by onset of labour and gestational age, NSW \& ACT 2000

Table 67: Confinements by onset of labour and birthweight, NSW \& ACT 2000

Table 68: Births by duration of rupture of membranes and gestational age, NSW \& ACT 2000

Table 69: Births by type of delivery and gestational age, NSW \& ACT 2000

Table 70: Births by type of delivery and birthweight, NSW \& ACT 2000

5.4 Infant characteristics

Table 71: Births by gestational age, NSW \& ACT 1996-2000

Figure 8: Births by gestational age, NSW \& ACT 2000

Table 72: Births by NICUS registration and gestational age, NSW \& ACT 2000

Table 73: Births by birthweight, NSW \& ACT 2000

Table 74: Births by NICUS registration and birthweight, NSW \& ACT 2000

Table 75: Births by gender and gestational age, NSW \& ACT 2000

Table 76: Births by congenital anomalies and gestational age, NSW \& ACT 2000

Table 77: Births by plurality and gestational age, NSW \& ACT 2000

Table 78: Births by Apgar score and gestational age, NSW \& ACT 2000

Table 79: Births by Apgar score at one and five minutes, NSW \& ACT 1996-2000

Table 80: Assisted ventilation and gestational age, NSW \& ACT 1996-2000

Figure 9: Births by main indication for assisted ventilation, NSW \& ACT 2000

Table 81: Main indication for assisted ventilation of babies by gestational age, NSW \& ACT 2000

Table 82: Proven systemic infection by gestational age, NSW \& ACT 2000

Table 83: Surfactant administration by gestational age, NSW \& ACT 1996-2000

Table 84: Treated patent ductus arteriosus (PDA) by gestational age, NSW \& ACT 2000

Table 85: Necrotising enterocolitis (NEC) by gestational age, NSW \& ACT 2000

Table 86: Major surgery by gestational age, NSW \& ACT 2000

Table 87: Intraventricular haemorrhage (IVH) by gestational age, NSW \& ACT 2000

Table 88: Retinopathy of prematurity by gestational age, NSW \& ACT 2000

5.5 Service utilisation

Figure 10: Median number of days in hospital, oxygen therapy and assisted ventilation by gestational age, NSW \& ACT 2000 
Figure 11: Total number of days in hospital, oxygen therapy and assisted ventilation by gestational age, NSW \& ACT 2000

Table 89: Service utilisation indicators by gestational age, NSW \& ACT 2000

Table 90: Home oxygen administration by gestational age, NSW \& ACT 1996-2000 69

5.6 Survival

Table 91: Duration of survival of babies by gestational age, NSW \& ACT 2000

Table 92: Duration of survival by birthweight, NSW \& ACT 2000

Table 93: Duration of survival by place of birth and gestational age, NSW \& ACT 2000

Table 94: Duration of survival by major congenital anomaly and gestational age, NSW \& ACT 2000

Figure 12: Deaths by post-mortem examination and gestational age, NSW \& ACT 1996-2000

Table 95: Post-mortem examination by gestational age, NSW \& ACT 2000

\section{Part 6: Birth Defects}

6.1 Birth defects among stillborn and liveborn infants $\quad 73$

$\begin{array}{ll}\text { 6.1.1 Trends in reported birth defects } & 73\end{array}$

6.1.2 Birth defects by diagnostic category 73

Table 96: $\quad$ Birth defect cases, NSW 1994-2000 73

Table 97: Birth defects among stillbirths and live births by diagnostic category, NSW 1994-2000

6.1.3 Infant characteristics

Table 98: Birth defect cases by gestational age, NSW 1994-2000

Table 99: Birth defect cases by pregnancy outcome, NSW 1994-2000

6.1.4 Maternal characteristics

6.2 Birth defects among terminations of pregnancy, spontaneous abortions and unknown outcomes of pregnancy

Table 100: Birth defect cases by maternal age, NSW 1994-2000

Table 101: Pregnancies with fetuses affected by birth defects and resulting in spontaneous abortion, termination of pregnancy or unknown outcome, NSW 1994-2000

Table 102: Birth defects among spontaneous abortions, terminations of pregnancy and unknown outcome of pregnancy by diagnostic category, NSW 1994-2000

Table 103: Trends in reported terminations of pregnancy associated with birth7 defects by maternal age, 1994-2000

6.3 Trends in selected birth defects

Figure 13: Neural tube defects: cases by year and pregnancy outcome, NSW 1994-2000

Figure 14: Cleft palate: cases by year and pregnancy outcome, NSW 1994-2000

Figure 15: Total cleft lip: cases by year and pregnancy outcome, NSW 1994-2000

Figure 16: Hypospadias: cases by year and pregnancy outcome, NSW 1994-2000

Figure 17: Limb reduction defects: cases by year and pregnancy outcome, NSW 1994-2000

Figure 18: Chromosomal abnormalities cases by year and pregnancy outcome, NSW 1994-2000

Figure 19: Down syndrome: cases by year and pregnancy outcome, NSW 1994-2000

Figure 20: Renal agenesis and dysgenesis: cases by year and pregnancy outcome, NSW 1994-2000 
7.1 Onset and augmentation of labour in selected hospitals 84

Table 105: Confinements by onset and augmentation of labour and hospital, NSW 200084

7.2 Type of delivery in selected hospitals 86

Table 106: Confinements by type of delivery and hospital, NSW $2000 \quad 86$

7.3 Pain relief in selected hospitals 88

Table 107: Confinements by type of pain relief and hospital, NSW 200088

7.4 Perineal status in selected hospitals 90

Table 108: Confinements with vaginal deliveries by perineal status and hospital, NSW 200090

$\begin{array}{lll}7.5 & \text { Birthweight in selected hospitals } & 92\end{array}$

Table 109: Births by birthweight and hospital, NSW 2000

7.6 Gestational age in selected hospitals 94

Table 110: Births by gestational age and hospital, NSW $2000 \quad 94$

7.7 Baby discharge status in selected hospitals 96

Table 111: Births by baby discharge status and hospital, NSW $2000 \quad 96$

7.8 Postnatal length of stay in selected hospitals 98

Table 112: Average maternal postnatal length of stay in hospital of birth, NSW 1995-1999 98

7.9 Indicators of obstetric care 99

Table 113: Clinical indicators for obstetrics, NSW and Australia $2000 \quad 99$

\section{Part 8: Review of Perinatal Deaths $2000 \quad 100$}

$\begin{array}{lll}8.1 & \text { Introduction } & 100\end{array}$

8.2 Perinatal death reviews, $2000 \quad 100$

8.3 Obstetric causes of perinatal death 100

Table 114: Perinatal deaths by obstetric cause and gestational age, NSW 2000

8.4 Obstetric causes of perinatal death by hospital size 103

Table 115: Perinatal deaths by obstetric cause and hospital size 103

8.5 Neonatal cause of death 103

Table 116: Neonatal deaths by cause and gestational age, NSW $2000 \quad 104$

8.6 Perinatal deaths associated with maternal drug dependency/abuse 103

8.7 Postmortem examination 103

8.8 Survey of perinatal death review procedures in NSW hospitals 105

Table 117: Type of perinatal death review by hospital size, NSW $2000 \quad 106$

$\begin{array}{ll}\text { Table 118: Coverage of births and perinatal deaths by type of } & \\ \text { perinatal death review, NSW } 2000 & 106\end{array}$

\section{Part 9: Risk-adjusted caesarean section rates in NSW hospitals 107}

$\begin{array}{lll}9.1 & \text { Introduction } & 107\end{array}$

$\begin{array}{ll}9.2 \text { Methods } & 107\end{array}$

$\begin{array}{llr}9.3 & \text { Results } & 107\end{array}$

9.4 Discussion 107

Table 119: Crude and adjusted odds ratios for caesarean section by clinical
risk factors found to be significant on logistic modelling

Table 120: Crude and adjusted caesarean section rates by hospital,

\section{Appendices}

Appendix 1: Description of selected birth defects 111

Appendix 2: Birth defect exclusion list 111

Appendix 3: Maternal countries of birth and country of birth groups 112

Appendix 4: Map of NSW health areas 113

Appendix 5: NSW Midwives Data Collection form $\quad 114$ 


\section{NEW SOUTH WALES MOTHERS AND BABIES 2000}

Prepared by: Lee Taylor, Margaret Pym, Barbara Bajuk, Lee Sutton, Susan Travis, Clare Banks and Kim Lim.

\section{INTRODUCTION}

This is the fourth report on mothers and babies in NSW to combine the annual reports of the NSW Midwives Data Collection (MDC), the Neonatal Intensive Care Units' Data Collection and the NSW Birth Defects Register. Information on causes of maternal deaths in NSW was obtained through the work of the NSW Maternal and Perinatal Committee and is also included.

From 1 January 1998, the MDC includes data elements necessary for most of the Australian Council on Healthcare Standards-Royal Australian and New Zealand College of Obstetricians and Gynaecologists (ACHS-RANZCOG) clinical indicators for obstetrics. A summary of the indicators for all NSW hospitals combined, and comparative information for participating Australian hospitals, is included in Part 7 of this report.

From 1 Janaury 2000, confidential reviews of perinatal deaths among babies of at least 22 weeks gestation or 500 grams birthweight are carried out by the NSW Maternal and Perinatal Committee. Part 8 describes the results of the review for deaths occurring in 2000, and the results of a survey of hospital perinatal death review practices in NSW.

In response to continuing concerns about rising caesarean section rates in NSW, a study was carried out to produce caesarean section rates for NSW hospitals adjusted for clinical risk factors. The results are described in Part 9.

\section{SUMMARY}

\section{Trends in NSW}

There were 87,922 babies born to 86,460 mothers in 2000 . The number of births in NSW remained stable at about 86,000 to 88,000 between 1996 and 2000. The number of teenage mothers decreased from 4,295 (5.0 per cent of all mothers) in 1996 to 3.853 (4.5 per cent) in 2000; while the number of mothers aged 35 years and over increased from 12,712 in 1996 to 15,334 in 2000 , an increase from 14.9 to 17.7 per cent of all confinements.

The reported number of Aboriginal and Torres Strait Islander mothers giving birth increased from 1,712 in 1996 (2.0 per cent of all mothers) to 2,105 in 2000 (2.4 per cent of all mothers). Part of this increase is likely to be due to an increased willingness of mothers to be identified as Aboriginal or Torres Strait Islander.

Patterns of maternal country of birth have remained fairly stable over the five year period with about one in four mothers born overseas in 2000, most commonly in the United Kingdom ( 3.0 per cent), China ( 2.5 per cent), Vietnam (2.4 per cent) and New Zealand (2.3 per cent).

The proportion of mothers planning to give birth in a birth centre reached a peak in 1996 (4.2 per cent) and subsequently fell slightly to 3.7 per cent in 2000 , while the reported number of mothers planning a home birth decreased from 247 to 146 over the five year period.
The rate of normal vaginal birth fell slightly from 70.7 per cent in 1996 to 67.1 per cent in 2000 . Over the five years, the caesarean section rate increased from 17.6 to 21.3 per cent and the rate of instrumental delivery remained steady at about 10.5 per cent.

Since 1995, the rate of low birthweight (less than 2,500 grams) was steady at about 6 per cent. The rate was 6.4 per cent in 2000 .

There was a slight increase in the percentage of babies born prematurely at 32-36 weeks gestation, and a decrease in the percentage born at 42 weeks or more. The overall rate of premature births (less than 37 weeks gestation) rose from 6.7 per cent in 1996 to 7.3 per cent in 2000 . There was no substantial change in the proportion of babies who were very premature (less than 32 weeks) or extremely premature (less than 28 weeks).

The perinatal mortality rate varied from 8.9 to 9.7 per 1,000 . About two-thirds of all perinatal deaths were stillbirths and one third were neonatal deaths.

In the period 1990-1998, 106 deaths were reported among pregnant women or women who gave birth less than six weeks previously. Seventy of these were classified as directly or indirectly associated with the pregnant state.

\section{Area Health Services}

In 2000, the largest numbers of births occurred among mothers resident in the Western Sydney and South Western Sydney Health Areas. These two areas contributed over one quarter of the state's births.

In 2000, as in previous years, there were large variations between health areas in the age distribution of women giving birth. The proportion of women giving birth at less than 20 years of age varied from 0.8 per cent in the Northern Sydney Health Area to 12.0 per cent in the Far West Health Area, while the proportion of mothers giving birth at 35 years of age or more ranged from 10.7 per cent in the Far West Health Area to 29.1 per cent in the Northern Sydney Health Area.

The proportion of Aboriginal or Torres Strait Islander mothers varied from 0.1 per cent in the Northern Sydney Health Area to 26.8 per cent in the Far West Health Area.

The highest proportions of mothers born in non-English speaking countries were in the Central Sydney and South Western Sydney Health Areas. In Central Sydney, the majority of mothers born in non-English speaking countries were born in North East Asia and South East Asia (12.2 and 10.7 per cent of all confinements respectively). In South Western Sydney, the majority of mothers born in non-English speaking countries were born in South East Asia (17.0 per cent of all confinements).

The highest rate of normal vaginal birth was among residents of Far West Health Area (76.4 per cent), while the highest rate of instrumental delivery was in South Eastern Sydney Health Area (15.0 per cent). 
The caesarean section rate varied from 16.0 per cent among mothers resident in the South Western Sydney Health Area to 26.5 per cent in the Northern Sydney and Mid Western Health Areas.

The highest rates of low birthweight occurred in the Macquarie Health Area (8.1 per cent) and the lowest rate occurred in the Southern Health Area (4.4 per cent). The highest rate of preterm birth was in the Mid Western Health Area (9.0 per cent). The lowest rate of preterm birth was 4.5 per cent in the Southern Health Area, which may result from referral of high risk pregnancies to the ACT.

The perinatal mortality rate in NSW in 2000 was 9.7 per 1,000 births. The rate varied from 5.6 per 1,000 in the Southern Health Area to 13.2 per 1,000 in the Hunter Health Area.

\section{Aboriginal and Torres Strait Islander mothers and babies}

In 2000, there were 2,122 babies reported to be born to Aboriginal and Torres Strait Islander mothers, 2.4 per cent of all babies born in NSW. About two thirds of Aboriginal and Torres Strait Islander mothers who gave birth in 2000 lived in rural areas. Almost one quarter lived in the New England or Macquarie Health Areas. About one in five Aboriginal and Torres Strait Islander mothers were teenagers. In 2000, 87.5 per cent of Aboriginal and Torres Strait Islander mothers were booked into the hospital of birth. This is lower than the 98.1 per cent of non-Aboriginal or Torres Strait Islander mothers who were booked into the hospital of birth in 2000.

In 2000, 67.6 per cent of Aboriginal and Torres Strait Islander mothers commenced antenatal care before 20 weeks gestation compared with 87.0 per cent of nonAboriginal and Torres Strait Islander mothers.

Following statewide trends, the rate of induction of labour among Aboriginal or Torres Strait Islander mothers increased from 16.6 to 17.7 per cent between 1996 and 2000 , while the rate of spontaneous onset of labour decreased from 76.8 to 72.5 per cent. However, the rate of induction of labour among Aboriginal and Torres Strait Islander mothers (17.7 per cent) continued to be lower than the statewide rate (23.6 per cent) in 1999. Also following statewide trends, the caesarean section rate among Aboriginal and Torres Strait Islander mothers rose from 16.0 to 18.2 per cent between 1996 and 2000 .

Since 1996, the rates of low birthweight (less than 2,500 grams) and prematurity (less than 37 weeks gestation) in Aboriginal and Torres Strait Islander babies have been over 10 per cent. These rates are one and a half times to two times higher than the rates for NSW overall. The perinatal mortality rate in babies born to Aboriginal and Torres Strait Islander mothers was 17.9 per 1,000 in 2000, over one and half times the rate of 9.7 per 1,000 for NSW overall.

\section{Maternal country of birth}

Between 1996 and 2000, about 20 per cent of mothers were born in non-English speaking countries. The proportion of mothers from Asian countries increased slightly from 10.5 to 11.6 per cent, while the proportion of mothers from Southern European countries decreased slightly from 1.8 to 1.4 per cent.

In 2000, the proportion of mothers born in non-English speaking countries was highest in the Central Sydney Health Area (44.7 per cent), followed by the South Western Sydney and Western Sydney Areas (40.3 and 36.0 per cent respectively).

Births to teenage mothers were less common among mothers born in non-English speaking countries than among mothers born in English speaking countries, as was smoking in pregnancy.

In 2000, 86.5 per cent of all mothers commenced antenatal care before 20 weeks gestation. There was some variation between country of birth groups, with 89.0 per cent of mothers born in English speaking countries commencing antenatal care before 20 weeks gestation, compared with 55.9 per cent of mothers born in Melanesia, Micronesia and Polynesia and 67.8 per cent of mothers born in the Middle East and Africa.

Mothers born in non-English speaking countries were more likely to have a spontaneous onset of labour than mothers born in English speaking countries and less likely to be induced.

Mothers born in Melanesia, Micronesia and Polynesia, and the Middle East and Africa were more likely to have a normal vaginal delivery than mothers in other country of birth groups. The highest caesarean section rates were among mothers born in Southern Asia (25.6 per cent) and Central and South America (25.1 per cent).

The highest rate of low birthweight was in babies of mothers born in Southern Asian countries (9.9 per cent). Babies of mothers born in North East Asia and Central and South America were least likely to be low birthweight.

The highest rate of prematurity was in babies of mothers born in Western and Northern Europe (8.9 per cent). Babies of mothers born in North East Asia or Central and South America were least likely to be premature.

Perinatal mortality rates varied substantially between country of birth groups, from 3.7 per 1,000 among babies of mothers born in North East Asia to 20.8 per 1,000 in babies of mothers born in Melanesia, Micronesia and Polynesia.

\section{Neonatal Intensive Care}

There were 2,003 infants registered in the Neonatal Intensive Care Units' Data Collection in 2000 representing a registration rate of 21.8 per 1,000 live births. Seventy nine (3.9 per cent) infants registered in 2000 were born to Aboriginal and Torres Strait Islander mothers. 
The 2,003 infants were born to 1,841 mothers, nearly 80 per cent of whom were residents of the Sydney, Central Coast, Hunter and Illawarra Health Areas. The age of mothers ranged from 15 to 53 years with a mean age of 28.9 years. Antenatal complications were reported for 87.3 per cent of mothers. The proportion of women receiving antenatal corticosteroids for lung maturation has increased each year since 1992 , with 70.0 per cent of mothers receiving steroids in 2000.

Thirty six per cent of infants registered in 2000 were born following a booked tertiary centre birth and 34.1 per cent were born following maternal transfer. Thirty per cent were transferred to a tertiary centre following birth and 4.7 per cent were transferred from one tertiary centre to another immediately after birth.

Nearly three quarters ( 74.7 per cent) of the infants registered in 2000 were born in a tertiary centre. There is an inverse relationship between gestational age and birth in a tertiary centre.

Boys comprised 58.6 per cent of the 2000 cohort and girls 41.4 per cent. Most infants (79.1 per cent) were from a singleton pregnancy, 18.8 per cent were from a twin pregnancy, 2.0 per cent were from a triplet pregnancy.

Seventy four per cent of infants registered during 2000 were preterm (less than 37 weeks gestation), 43.9 per cent were very preterm (less than 32 weeks gestation) and 13.7 per cent were extremely preterm (less than 28 weeks gestation). Nearly one in five (19.6 per cent) infants had a major or minor congenital anomaly.

Infants with major congenital anomalies were excluded from the analysis of mortality and morbidity. The majority of infants registered in 2000 (88.7 per cent) received assisted ventilation (intermittent mandatory ventilation or continuous positive airways pressure ventilation). The main indication for assisted ventilation varied with gestational age: respiratory distress syndrome, immature lung and transient tachypnoea were more common among preterm groups, whereas meconium aspiration and perinatal asphyxia were more common in term infants.

Proven systemic infection was present in 14.4 per cent of infants, necrotising enterocolitis in 2.2 per cent, intraventricular haemorrhage in 15.2 per cent, treated patent ductus arteriosus in 15.4 per cent, and major surgery in 3.0 per cent. Severe grades (Grade 3 or 4 ) of retinopathy of prematurity were present in 4.8 per cent of infants less than 32 weeks gestation, of whom 75.0 per cent had either cryo or laser therapy to prevent retinal detachment. Surfactant was given to 42.6 per cent of infants; the majority (66.7 per cent) of ventilated infants with a diagnosis of Respiratory Distress Syndrome received surfactant.

Overall, 92.5 per cent of infants without a major congenital anomaly survived to six-months of age. Survival improved with gestational age up to 32 weeks after which it decreased slightly. Of the infants who died, most (67.5per cent) died at less than one week of age and a further 17.5 per cent died at less than 29 days of age. The six-month survival rate for infants born at 23 to 27 weeks gestation was higher for those born in a non-tertiary centre $(90.5$ per cent) compared with those born in a tertiary centre (73.1 per cent). This result should be interpreted with extreme caution. Among infants born at higher gestational ages the proportion surviving to six-months of age was similar for those born in a tertiary centre and those born in a non-tertiary centre.

\section{Birth defects}

About 2,000 infants are born with birth defects each year in NSW, and for about half of these infants the malformation is detected after birth. In 1994-2000, defects of the cardiovascular system were most commonly reported, followed by defects of the musculoskeletal system and defects of the genito-urinary system. This is a similar pattern to previous years. In 1999, the overall rate of defects was slightly lower than the previous five years combined (39.1 versus 42.9 per 1,000), due to a decrease in the number of babies with multiple malformations. The proportion of reported cases with three or more malformations fell from 21.4 to 18.90 per cent between 1994 and 1999.

In the period 1994-1998, about 150 terminations of pregnancy per year were reported to the NSW Birth Defects Register. Following the introduction of a requirement to notify birth defects under the NSW Public Health Act 1991 from 1 January 1998, the number of terminations reported rose to 250 in 1998 and 308 for 1999.

Of the total 1,222 terminations of pregnancy reported in 1994-2000, 706 (57.8 per cent) were associated with a chromosomal abnormality, the most common of which was Down syndrome, and 276 (22.6 per cent) were associated with a neural tube defect.

Birth defects were more common among premature infants compared to full term infants, and among male infants compared to female infants. The rate of birth defects increases with increasing maternal age, especially after age 35 . However, as most babies are born to mothers aged less than 35 years, the majority of babies with birth defects were born to younger mothers. 


\section{DATA SOURCES}

\section{The New South Wales Midwives Data Collection}

The New South Wales Midwives Data Collection (MDC) is a population-based surveillance system covering all births in NSW public and private hospitals, as well as home births. It encompasses all livebirths and stillbirths of at least 20 weeks gestation or at least 400 grams birth weight.

The MDC relies on the attending midwife or doctor to complete a notification form when a birth occurs. The form, a copy of which is shown at Appendix 4, includes demographic items and items on maternal health, the pregnancy, labour, delivery and perinatal outcomes. Completed forms are sent to the Patient Data Management Unit of the Information Management and Clinical Systems Branch of the NSW Health Department, where they are compiled into the MDC database.

The MDC receives notifications of women whose usual place of residence is outside NSW but who give birth in NSW. However, the MDC does not receive notifications of births outside NSW to women usually resident in NSW.

\section{The Neonatal Intensive Care Units' Data Collection}

The Neonatal Intensive Care Units' (NICUS) Data Collection is a statewide audit of infants admitted to neonatal intensive care units in New South Wales (NSW) and the Australian Capital Territory (ACT) during the neonatal period for one of the following reasons:

- gestational age less than 32 weeks;

- birth weight less than or equal to 1,500 grams;

- mechanical ventilation for four hours or more;

- continuous positive airways pressure (CPAP) for four hours or more;

- major surgery (opening of a body cavity).

In 2000 the 10 neonatal intensive care units in NSW and ACT were situated at the following perinatal centres: John Hunter Children's Hospital-Newcastle, King George V Hospital, Liverpool Health Service (joined 12/10/94), Nepean Hospital, Royal Hospital for Women, Royal North Shore Hospital, The Canberra Hospital-Woden Valley (joined 1/1/95), Westmead Hospital, and at the two paediatric hospitals: Sydney Children's Hospital and Royal Alexandra Hospital for Children (The Children's Hospital at Westmead).

The neonatal, maternal and perinatal data which comprise the NICUS Data Collection are collected and collated within each neonatal intensive care unit by a designated Clinical Audit Officer. The data are compiled into a central database located at the NSW Centre for Perinatal Health Services Research.

\section{The New South Wales Birth Defects Register}

The NSW Birth Defects Register (BDR) is a populationbased surveillance system established to monitor birth defects detected during pregnancy or at birth, or diagnosed in infants up to one year of age. The BDR was established in 1990 and under NSW Public Health Act 1991 from 1 January 1998 doctors, hospitals and laboratories have been required to notify birth defects detected during pregnancy, at birth, or up to one year of life . The BDR is administered by the Epidemiology and Surveillance Branch of the NSW Health Department.

The activities of the BDR include: annual publication of information on birth defects in NSW; provision of information to area health services to assist in service planning and monitoring of child health, and investigation of specific issues; provision of information in response to specific requests from the public, health professionals, and other government departments; and provision of data to the AIHW National Perinatal Statistics Unit (NPSU) for monitoring of birth defects at a national level. The NPSU is also responsible for providing Australian information on birth defects to the International Clearinghouse for Birth Defects Monitoring Systems, a non-governmental organisation of the World Health Organization.

Sources of notifications to the BDR include: the NSW Midwives Data Collection (MDC), specialist paediatric hospitals, cytogenetic laboratories, and individual health care providers. The BDR is supported by an advisory committee, comprising a panel of clinical experts representing the following specialities: genetics, dysmorphology, neonatology, obstetrics and gynaecology, midwifery, bioethics and epidemiology; and a community representative from the Association of Genetic Support of Australasia.

Data for research purposes may be provided in two formats: aggregate information similar to that contained in this report, and data concerning individuals with identifying information removed. All requests for data should be submitted in writing to the Director, Epidemiology and Surveillance Branch. Requests for data concerning individuals for sufficiently important research purposes will be referred to the Statewide Health Confidentiality and Ethics Committee. Procedures for release of personal information are described in the Department's Information Privacy Code of Practice, copies of which are available through the NSW Health Department's World Wide Web site at www.health.nsw.gov.au.

\section{The NSW Inpatient Statistics Collection}

For this report data from the NSW Inpatient Statistics Collection (ISC) was linked to MDC data to produce information on postnatal length of stay in NSW hospitals, and, from 1998 health insurance status.

The ISC covers demographic and episode related data for every inpatient who is separated from any public, private, and repatriation hospital, private day procedure centre or public nursing home in NSW. Separation can result from discharge, transfer, death, or change in service category. The ISC is maintained by the NSW Health Department's Information Management and Clinical Systems Branch. 


\section{NSW Maternal and Perinatal Committee}

The NSW Maternal and Perinatal Committee is a quality assurance committee established under the Health Administration Act 1982, and is privileged under the Act to carry out confidential reviews of both maternal and perinatal deaths. Members are appointed by the Minister for Health. The committee reviews each maternal death to identify any possible avoidable factors and to determine whether the death was related to pregnancy (or its management) or whether it was incidental. The committee also reviews perinatal deaths of at least 22 weeks gestation or at least 500 grams birthweight. The information obtained from these reviews assists in the development of policies aimed at improving the health of mothers and newborns in NSW. Information considered by the Committee is confidential.

\section{DEFINITIONS}

\section{Aboriginal and Torres Strait Islander}

Women who identify themselves to be of Australian Aboriginal and Torres Strait Islander heritage.

\section{Apgar score}

A numerical scoring system routinely administered one and five minutes after birth to evaluate the condition of the baby. The score ranges from 0-10 (10 being perfect). It takes account of five physical signs, each of which is assigned a component score of 0,1 or 2 : heart rate, respiration, muscle tone, reflexes and colour.

\section{Augmentation}

Artificial rupture of the membranes or use of oxytocic drugs after spontaneous onset of labour.

\section{Birth defect}

Any structural defect or chromosomal abnormality detected during pregnancy, at birth, or in the first year of life, excluding birth injuries and minor anomalies such as skin tags, talipes, birthmarks or clicky hips. Descriptions of some of the birth defects included in this report are shown in Appendix 1, and a list of common exclusions used by the NSW Birth Defects Register is shown in Appendix 2. From 1994, the following conditions were included in the NSW Birth Defects Register: congenital hypothyroidism, cystic fibrosis, phenylketonuria and thalassaemia major.

\section{Birthweight}

The newborn infant's first bare weight in grams.

Low birthweight: birth weight less than 2,500 grams.

Very low birthweight: birth weight less than 1,500 grams.

Extremely low birthweight: birth weight less than 1,000 grams.

\section{Caesarean section}

Delivery of the fetus through an abdominal incision.
Elective caesarean section: a caesarean section (planned or unplanned) performed before the onset of labour.

Emergency caesarean section: a caesarean section performed after the onset of labour, whether or not the onset of labour was spontaneous.

\section{Confinement}

Refers to a woman having given birth. In a multiple pregnancy, one confinement will result in more than one birth.

\section{Country of birth}

The mother's country of birth.

\section{Epidural}

Injection of analgesic agent outside the dura mater which covers the spinal canal; includes lumbar, spinal and epidural anaesthetics.

\section{Episiotomy}

An incision of the perineum and vagina to enlarge the vulval orifice.

\section{Gestational age}

The duration of pregnancy in completed weeks from the first day of the last normal menstrual period. Where accurate information on the date of the last menstrual period is not available, a clinical estimate of gestational age may be obtained from ultrasound during the first half of pregnancy or by examination of the newborn infant. The 'best estimate' is used here.

\section{Induction of labour}

Oxytocics-prostaglandins: the initiation of labour by the use of oxytocic agents, prostaglandins, or their derivatives (oral, intravaginal or intravenous).

ARM only: the initiation of labour by artificial rupture of membranes.

Oxytocics-prostaglandins and ARM: both medical and surgical induction as defined above (combined medical and surgical induction).

\section{Intraventricular haemorrhage (IVH)}

Worst level of intraventricular haemorrhage (IVH) seen on either right or left side by either ultrasound or postmortem examination.

None: ultrasound-post-mortem shows no

Grade 1: haemorrhage

Grade 2: $\quad$ intraventricular haemorrhage with no

Grade 3: $\quad$ intraventricular haemorrhage with

Grade 4: ventricle distended with blood

Not examined: No ultrasound or post-mortem examination. 


\section{Live birth}

The complete expulsion or extraction from its mother of a baby of at least 400 grams or 20 weeks gestation who, after being born, breathes or shows any evidence of life such as a heartbeat.

\section{Major surgery}

Any surgery that requires opening of a body cavity.

\section{Mechanical ventilation}

Use of a mechanical ventilator to provide intermittent positive pressure respiration for a baby for four hours or more.

\section{Necrotising enterocolitis (NEC)}

Clinically diagnosed: received treatment for NEC (includes suspending feeds, blood cultures and treatment with antibiotics such as clindamycin-gentamycin).

Proven radiologically or at operation: radiological signs include intra-mural or intra-hepatic air, perforation or a 'fixed loop'.

\section{Neonatal death}

The death of a live born infant within 28 days of birth.

\section{Neonatal period}

The first 28 completed days of life.

\section{Neonatal mortality rate}

The number of neonatal deaths per 1,000 live births.

\section{Patent ductus arteriosus (PDA)}

Clinical signs of PDA such as typical murmur, active precordium, bounding pulses, cardiomegaly, or pulmonary vascular congestion on X-ray. May be confirmed on ultrasound examination.

\section{Parity}

The total number of live births and stillbirths of the mother before the pregnancy or birth under consideration.

\section{Perinatal death}

A stillbirth or neonatal death.

\section{Perinatal mortality rate}

The number of perinatal deaths (stillbirths and neonatal deaths) per 1,000 total births in a year (livebirths and stillbirths combined).

\section{Perineal status}

1st degree tear: a perineal graze-laceration-tear involving: the fourchette, hymen, labia, skin, vagina or vulva.

2nd degree tear: a perineal laceration or tear involving the pelvic floor or perineal muscles or vaginal muscles. 3rd degree tear:

a perineal laceration-tear involving the anal sphincter or rectovaginal septum.

4th degree tear: $\quad$ a third degree perineal laceration or tear which also involves the anal mucosa or rectal mucosa.

\section{Plurality}

The number of fetuses or babies from the pregnancy. On this basis pregnancy may be classified as single or multiple.

\section{Premature infant}

An infant born before 37 completed weeks gestation.

\section{Premature labour}

The spontaneous onset of labour (regular painful contractions with progressive cervical changes) before 37 completed weeks of gestation.

\section{Retinopathy of prematurity}

Worst stage of retinopathy of prematurity (ROP) in either eye during the initial hospital admission.

None seen: no changes seen

Stage I: demarcation line present

Stage II: ridge present

Stage III: ridge with extra-retinal fibrovascular proliferation

Stage IV: retinal detachment

\section{Systemic infection in the infant}

Clinical or radiological signs of infection together with growth of a known pathogen from a systemic site-does not include tracheal aspirate.

\section{Transfer (NICUS only)}

Maternal transfer before birth (prenatal): the transfer of a pregnant woman to a tertiary obstetric hospital.

Neonatal transfer after birth (postnatal): the transfer of an infant from the hospital of birth to a tertiary NICU.

\section{Spontaneous abortion}

The spontaneous expulsion of a fetus less than 20 weeks gestation and less than 400 grams birthweight.

\section{Stillbirth}

The complete expulsion or extraction from its mother of a product of conception of at least 20 weeks gestation or 400 grams birthweight who did not, at any time after delivery, breathe or show any evidence of life such as a heartbeat.

\section{Termination of pregnancy}

A procedure intentionally performed to terminate a pregnancy before 20 completed weeks gestation. 


\section{EXPLANATORY NOTES}

\section{Antenatal complications (NICUS)}

These specifically include antepartum haemorrhage, placenta praevia, placenta abruptio, prolonged rupture of membranes, gestational diabetes, threatened preterm labour, hypertensive disease of pregnancy and rhesus isoimmunisation. There is also an open-ended 'other antenatal complications' option. The most common problems specified in this option are cervical incompetence, polyhydramnios, oligohydramnios, chorioamnionitis, threatened miscarriage and problems secondary to multiple pregnancy.

\section{Rates of birth defects}

The BDR collects data pertaining to birth defects regardless of the outcome of pregnancy. This includes notifications of livebirths, stillbirths, terminations of pregnancy and spontaneous abortions. Birth defect rates are calculated using births (that is, livebirths and stillbirths) as the denominator, because denominator populations for pregnancies less than 20 weeks gestation are unknown. The numerators are described in the relevant sections.

The source of denominator population data on births is the MDC. The MDC was selected because its definitions are consistent with those applied by the BDR.

Denominator populations compatible with the BDR were derived from the MDC by including only those births that occurred to NSW residents.

Caution should be exercised when comparing the birth defect rates tabled in this document with those reported within the NPSU's Congenital Malformations Australia Report. This report covers birth defects detected during pregnancy and up to one year of age while the Congenital Malformations Australia Report covers birth defects detected during pregnancy and up to 28 days of life.

Variations in data published by the BDR and interstate birth defects registers may be due to differences in coding practices, in categories of birth defects included in each Register and differences in the upper age limit for notification of cases.

\section{Place of residence of mother}

The mother's usual residence was the basis for coding to statistical local areas and NSW health areas.

\section{Labour}

The category 'labour-spontaneous with oxytocicsprostaglandins' was used where labour was augmented with artificial rupture of membranes as well as oxytocics or prostaglandins.

\section{Levels of neonatal care Tertiary}

Level 3: Neonatal Intensive Care Unit (NICU) — a unit that provides high-dependency specialist nursing and medical care for all newborn infants including sustained 'life support' such as mechanical ventilation and has staff neonatologists and neonatal registrars.

\section{Non-tertiary}

Level 2a: Neonatal Care-a unit which can give highlevel oxygen, can start mechanical ventilation if necessary and has paediatric house staff.

Level $2 b$ : Neonatal Care-a unit which can give low-level oxygen and has a paediatrician on call.

\section{Level of obstetric hospitals}

Level 1: local hospitals (no births), postnatal only.

Level 2: small isolated hospitals, low-risk births only. Staffed by general practitioners and midwives.

Level 3: country district and smaller metropolitan hospitals, care for mothers and infants at low-moderate risk. Full resuscitation and theatre facilities available. Rostered obstetricians, resident medical staff and midwives. Accredited general practitioners-specialist anaesthetist on call. Has Level $2 \mathrm{~b}$ neonatal care.

Level 4: country base-metropolitan district hospitals. Delivery and care for mothers and/or babies with moderate risk factors. Obstetricians and paediatrician available 24 hours a day, seven days a week. Rostered resident medical staff, specialist anaesthetist on call. Has Level $2 b$ neonatal care.

Level 5: country base-metropolitan district hospitals, care for mothers and infants known to be at high risk. Able to cope with complications arising from these risk factors. Has Level 2a neonatal care.

Level 6: (tertiary)—specialist obstetric hospitals (supra regional). All functions-low, moderate and high-risk births. Has Level 3 neonatal intensive care.

\section{Type of delivery}

The 'vaginal breech' category covers all forms of vaginal breech delivery, including forceps to the after-coming head.

\section{Perinatal mortality rate}

Perinatal deaths include deaths reported to the MDC only. As the MDC form is completed at discharge or transfer of the baby, deaths occurring after this time may not be reported to the MDC. Birth and perinatal death registration data held by the Australian Bureau of Statistics (ABS) give the most complete ascertainment of perinatal deaths for calculation of rates. 


\section{METHOD FOR ESTIMATING LEVEL OF REPORTING OF MATERNAL ABORIGINALITY (PART 3)}

The Aboriginality of the mother, rather than the baby, is reported to the MDC, although mother's Aboriginality is frequently used as a proxy measure for the baby's Aboriginality. Consequently, maternal Aboriginality was used for this analysis.

Aboriginal or Torres Straight Islander mothers were counted as one group in the MDC up to 1997 and as two separate groups thereafter. We were therefore unable to examine trends in the quality of reporting for both these groups.

Records of births reported to the MDC were linked to birth registration records of the NSW Registry of Births, Deaths and Marriages for births occurring in the six-year period 1994-1999. Records from the two files were matched using a probabilistic linkage software (Automatch). Prior to matching, residential address and mothers' name were standardised using a standardisation software (Autostan). The overall linkage rate was 96.6 per cent of MDC records (97.8 per cent of birth registration records).

Capture-recapture methods are used to adjust estimates of counts to reflect ascertainment level or undercounting. Capture-recapture was carried out using the method described by McCarty et al. ${ }^{1}$ Analysis was carried out using $\mathrm{SAS}$ version 8.02. Analyses concerning geographic location were based on health area of hospital of birth as reported to the MDC. Home births and births for which the hospital of birth was not stated were excluded from the analysis.

\section{References}

1. McCarty DJ, Tull ES, Moy CS, Kwoh CK, LaPorte RE. Ascertainment corrected rates: Applications of CaptureRecapture Methods. Int J Epidemiol 1993; 22(3): 559-565.

\section{METHOD FOR CALCULATION OF RISK- ADJUSTED CAESAREAN SECTION RATES (PART 9)}

Data were obtained from the MDC for the year 2000. The data set was divided into two parts based on baby's date of birth. Logistic regression models were developed on records of babies born between 1 January and 30 June. The model was then validated using the data on births occurring in the second half of the year.

Originally logistic models were developed using data for 1999. We found some differences in caesarean rates by gestational age in 1999 compared to 2000. Caesarean rates were higher at 28 weeks gestation and much lower after 40 weeks gestation in 2000 compared to 1999 . As there was sufficient power in half a year of data to both develop and validate the logistic model, we decided to use only the 2000 data for the study.

Cases selected were mothers who had confinements in NSW hospitals. Records with values recorded as not stated in variables that were risk factors for caesarean section were excluded from the analysis, as were records of mothers who delivered at less than 20 weeks gestation.

Crude odds ratios were calculated for clinical risk factors. Multiple logistic regression models were developed. In the logistic regression models, the outcome was the probability of having a caesarean section and risk factors were maternal age, presentation (categorised as breech, other malpresentation and vertex), parity (primiparous mothers or multiparous mothers), multiple birth, diabetes mellitus, essential hypertension, gestational diabetes, pregnancy induced hypertension, previous caesarean section and gestational age (categorised as 20-28 weeks, 29-39 weeks and 40+ weeks). Age was included in the model as a proxy measure for medical conditions and obstetric complications for which specific information was not available. The choice of variables to include in the models was based on a $p$-value of 0.05 for main effects and $p$-value of 0.001 for interaction terms. The referent levels were vertex presentation, multiparous mothers, singleton births, absence of diabetes mellitus or essential hypertension or gestational diabetes or pregnancy induced hypertension, mothers who did not have a previous caesarean section and 40+ weeks gestational age.

Departures from linearity for age and gestational age were tested using the Box-Tidwell Transformation. ${ }^{1}$ Adequacy of the logistic regression models was assessed using likelihood ratio tests, $c$ statistic (model discriminatory performance) and Hosmer-Lemeshow goodness of fit test. ${ }^{1}$ The $c$ statistic is equivalent to the area under the Receiving Operating Characteristic (ROC) curve.

The Box-Tidwell transformation on age indicated that the relationship between age and the logit of caesarean section was linear. Box-Tidwell transformation on gestational age showed that the logit of caesarean section is a non-linear function of gestational age. Age was entered as a continuous variable and gestational age was entered as a categorical variable in the logistic regression models.

Variables found to have an independent significant association with the probability of having a caesarean section (as opposed to other type of delivery) were age, presentation, multiple births, diabetes mellitus, essential hypertension, pregnancy induced hypertension, previous caesarean section and gestational age. The choice between competing statistical models gives preference to models that are simple to interpret and which adequately describe the data. In this situation the model with significant interactions gave a better fit (Hosmer-Lemeshow goodness of fit statistic with $8 d f=3.65, p=0.8872$ ). The $c$ statistic (area under the ROC curve) for the model was 0.852 .

Significant interaction terms in the final model were primiparous mothers by age, presentation by gestational age, primiparous mothers by gestational age, essential hypertension by gestational age, previous caesarean section by gestational age, and previous caesarean section by presentation. 
The logistic regression model was validated on the second half of MDC 2000 data. We constructed ROC curves by calculating model sensitivity and specificity for predicted probabilities across the range 0 to 1 and calculating the Hosmer-Lemeshow goodness of fit statistic. Area under the ROC curve is a measure of refinement, that is, how well the model can discriminate between positive and negative outcomes. ${ }^{2}$ We used the Hosmer-Lemeshow statistic as a measure of calibration. Calibration or reliability shows how well the predicted probabilities agree with responses in the validation data.

The generated ROC curve indicated that the model shows good predictive power and a good measure of refinement in the validation data (area under the ROC curve $=0.853$ ). Results of the Hosmer-Lemeshow goodness-of-fit test indicated a poor fit to the validation data (HosmerLemeshow statistic $=48.77$ with $10 d f, p=0.000$ ). This is most likely due to incomplete calibration because of a lower prevalence of caesarean section in the development data compared to the validation data. We then applied Cox's approach to assessing measures of calibration and refinement. ${ }^{2}$ This approach uses a logistic model on the logit of predicted probabilities from the development data set. The overall test for reliability of prediction is $\mathrm{H}_{\mathrm{o}}: \alpha=0$ and $\beta=1$. The estimates for the model we developed were $\alpha=0.098$ and $\beta=1.013$ (95\% confidence interval 0.99 1.03). Based on these results the model is adequate for predicting caesarean section rates in NSW hospitals for the period July-December 2000.

Predicted probabilities from the final logistic model were applied to individual records for hospitals with 100 or more births during July-December 2000, resulting in each confinement being allocated a probability of caesarean section. The probabilities were summed for each hospital to give the expected number of caesarean sections by hospital, adjusted for the risk factors in the model. Standardised caesarean ratios were calculated for hospitals by calculating the ratio of observed to expected number of caesarean sections. The risk-adjusted caesarean section rate for each hospital was calculated by multiplying its standardised caesarean ratio with the caesarean section rate in the standard population of births (20.8 per cent for the first six months of 2000).

\section{References}

1. Hosmer DW, Lemeshow S. Applied logistic regression. New York: Wiley, 1989.

2. Miller ME, Hui SL, Tierney WM. Validation techniques for logistic regression models. Statistics in Medicine 1991;10:1213-1226.

\section{ACKNOWLEDGEMENTS}

The NSW Midwives Data Collection, depends entirely on the state's midwives who provide information on each birth. We gratefully acknowledge their contribution.We would like to thank Perinatal Death Review Committees and clinical staff who completed Confidential Report on
Perinatal Death forms, and staff who completed the survey questionnaire on hospital perinatal death review practices.

The production of the NICUS chapter of this report was accomplished by the teamwork of a vast number of individuals and institutions. We thank the directors and clinical audit officers of the 10 neonatal intensive care units and the liaison officers representing all of the obstetric hospitals in NSW and ACT. Finally, and not least, the families who participated in the audit are acknowledged and thanked for their continued patience and support.

The NSW Birth Defects Register is dependent on families, health care institutions, and individual health care providers, for the supply of information about birth defects. We thank health information managers in hospitals throughout NSW for their cooperation and assistance. The staff of the medical record departments at The Children's Hospital at Westmead, The Sydney Children's Hospital and The John Hunter Hospital have also provided invaluable assistance.

We would also like to express our appreciation to members of the NSW Maternal and Perinatal Committee and the NSW Birth Defects Register Advisory Committee for their advice and support.

\section{FURTHER INFORMATION}

Director

Epidemiology and Surveillance Branch

NSW Department of Health

Locked Bag 961

North Sydney NSW 2059

Telephone: 0293919676

Facsimile: 0293919232

Manager

NSW Midwives Data Collection

Patient Data Management Unit

Information Management and Support Branch

NSW Department of Health

Locked Bag 961

North Sydney NSW 2059

Telephone: 0293919199

Facsimile: 0293919070

Director

Neonatal Intensive Care Units' Data Collection

NSW Centre for Perinatal Health Services Research

Queen Elizabeth II Institute for Mothers and Babies, D02

University of Sydney NSW 2006

Telephone: 0293517751

Facsimile: 0293517742

Manager

NSW Birth Defects Register

Epidemiology and Surveillance Branch

NSW Department of Health

Locked Bag 961

North Sydney NSW 2059

Telephone: 0294245829

Facsimile: 0293919232 


\section{PART 1: TRENDS IN NEW SOUTH WALES}

\subsection{CONFINEMENTS AND BIRTHS BY PLURALITY}

The number of births per year has remained fairly stable over the past five years (Table 1). There were 87,922 births to 86,460 women reported to the MDC for 2000 . Of the 86,460 confinements reported in 2000, 1,404 (1.6 per cent) were for twins and 29 for triplets.

\section{TABLE 1}

BIRTHS AND CONFINEMENTS BY PLURALITY, NSW 1996-2000

\begin{tabular}{|c|c|c|c|c|c|c|c|c|c|c|}
\hline \multirow[t]{2}{*}{ Plurality } & \multicolumn{4}{|c|}{1996} & \multicolumn{2}{|c|}{$\begin{array}{l}\text { Year } \\
1998\end{array}$} & \multicolumn{2}{|c|}{1999} & \multicolumn{2}{|c|}{2000} \\
\hline & No. & $\%$ & No. & $\%$ & No. & $\%$ & No. & $\%$ & No. & $\%$ \\
\hline \multicolumn{11}{|l|}{ Confinements } \\
\hline Singleton & 84201 & 98.7 & 85740 & 98.6 & 83869 & 98.6 & 84676 & 98.5 & 85027 & 98.3 \\
\hline Twins & 1076 & 1.3 & 1147 & 1.3 & 1174 & 1.4 & 1261 & 1.5 & 1404 & 1.6 \\
\hline Triplets & 24 & 0.0 & 32 & 0.0 & 28 & 0.0 & 30 & 0.0 & 29 & 0.0 \\
\hline Quadruplets & 1 & 0.0 & 1 & 0.0 & 1 & 0.0 & 0.0 & 0.0 & 0.0 & 0.0 \\
\hline Total & 85302 & 100.0 & 86920 & 100.0 & 85072 & 100.0 & 85967 & 100.0 & 86460 & 100.0 \\
\hline \multicolumn{11}{|l|}{ Births } \\
\hline Singleton & 84201 & 97.4 & 85740 & 97.3 & 83869 & 97.2 & 84676 & 97.0 & 85027 & 96.7 \\
\hline Twins & 2152 & 2.5 & 2293 & 2.6 & 2348 & 2.7 & 2523 & 2.9 & 2808 & 3.2 \\
\hline Triplets & 72 & 0.1 & 96 & 0.1 & 84 & 0.1 & 90 & 0.1 & 87 & 0.1 \\
\hline Quadruplets & 4 & 0.0 & 4 & 0.0 & 4 & 0.0 & 0.0 & 0.0 & 0.0 & 0.0 \\
\hline Total & 86429 & 100.0 & 88133 & 100.0 & 86305 & 100.0 & 87289 & 100.0 & 87922 & 100.0 \\
\hline
\end{tabular}

Source: NSW Midwives Data Collection (HOIST). Epidemiology and Surveillance Branch, NSW Department of Health.

\subsection{HEALTH AREA OF RESIDENCE}

Over the last five years, there was in increase in mothers giving birth in the Northern Sydney and South Eastern Sydney health areas, accompanied by a slight decline in most rural heath areas (Table 2). Births reported in the Greater Murray Health Area decreased from 1998 due to the closure of the obstetric unit at the Mercy Care Centre Albury in June 1998, and referral of women to Wodonga Hospital in Victoria.

\section{TABLE 2}

CONFINEMENTS BY HEALTH AREA OF RESIDENCE, NSW 1996-2000

\begin{tabular}{|c|c|c|c|c|c|c|c|c|c|c|}
\hline \multirow[t]{2}{*}{ HealthArea } & \multicolumn{2}{|c|}{1996} & \multicolumn{2}{|c|}{1997} & \multicolumn{2}{|c|}{$\begin{array}{l}\text { Year } \\
1998\end{array}$} & \multicolumn{2}{|c|}{1999} & \multicolumn{2}{|c|}{2000} \\
\hline & No. & $\%$ & No. & $\%$ & No. & $\%$ & No. & $\%$ & No. & $\%$ \\
\hline Central Sydney & 6839 & 8.0 & 6657 & 7.7 & 6574 & 7.7 & 6625 & 7.7 & 6775 & 7.8 \\
\hline Northern Sydney & 8552 & 10.0 & 8985 & 10.3 & 8824 & 10.4 & 9242 & 10.8 & 9432 & 10.9 \\
\hline Western Sydney & 10250 & 12.0 & 10559 & 12.1 & 10541 & 12.4 & 10712 & 12.5 & 10794 & 12.5 \\
\hline Wentworth & 4836 & 5.7 & 4827 & 5.6 & 4825 & 5.7 & 4851 & 5.6 & 4921 & 5.7 \\
\hline South Western Sydney & 12034 & 14.1 & 12511 & 14.4 & 12050 & 14.2 & 12219 & 14.2 & 12541 & 14.5 \\
\hline Central Coast & 3677 & 4.3 & 3792 & 4.4 & 3736 & 4.4 & 3665 & 4.3 & 3772 & 4.4 \\
\hline Hunter & 6997 & 8.2 & 7034 & 8.1 & 6875 & 8.1 & 6965 & 8.1 & 6981 & 8.1 \\
\hline Illawarra & 4320 & 5.1 & 4434 & 5.1 & 4350 & 5.1 & 4413 & 5.1 & 4407 & 5.1 \\
\hline South Eastern Sydney & 8706 & 10.2 & 9148 & 10.5 & 9135 & 10.7 & 9428 & 11.0 & 9697 & 11.2 \\
\hline Northern Rivers & 2956 & 3.5 & 2963 & 3.4 & 2941 & 3.5 & 2903 & 3.4 & 2766 & 3.2 \\
\hline Mid North Coast & 3038 & 3.6 & 3073 & 3.5 & 2954 & 3.5 & 2906 & 3.4 & 2802 & 3.2 \\
\hline New England & 2472 & 2.9 & 2463 & 2.8 & 2381 & 2.8 & 2348 & 2.7 & 2265 & 2.6 \\
\hline Macquarie & 1734 & 2.0 & 1595 & 1.8 & 1589 & 1.9 & 1596 & 1.9 & 1590 & 1.8 \\
\hline Mid Western & 2355 & 2.8 & 2437 & 2.8 & 2339 & 2.7 & 2297 & 2.7 & 2264 & 2.6 \\
\hline Far West & 568 & 0.7 & 600 & 0.7 & 556 & 0.7 & 533 & 0.6 & 533 & 0.6 \\
\hline Greater Murray & 3440 & 4.0 & 3357 & 3.9 & 2946 & 3.5 & 2603 & 3.0 & 2517 & 2.9 \\
\hline Southern & 1812 & 2.1 & 1776 & 2.0 & 1782 & 2.1 & 1845 & 2.1 & 1766 & 2.0 \\
\hline Other-Not stated & 716 & 0.8 & 709 & 0.8 & 674 & 0.8 & 816 & 0.9 & 637 & 0.7 \\
\hline TOTAL & 85302 & 100.0 & 86920 & 100.0 & 85072 & 100.0 & 85967 & 100.0 & 86460 & 100.0 \\
\hline
\end{tabular}

Source: NSW Midwives Data Collection (HOIST). Epidemiology and Surveillance Branch, NSW Department of Health. 


\subsection{MATERNAL AGE}

The number of teenage mothers decreased from 4,295 in 1996 to 3,853 in 2000 (Figure 1, Table 3). The mean maternal age rose slightly from 29.2 to 29.8 years over the same period.

The number of mothers 35 years of age or over giving birth increased from 12,712 in 1996 to 15,334 in 2000, an increase from 14.9 to 17.7 per cent of all confinements.
The trend towards later childbirth is evident among both primiparous and multiparous mothers: the proportion of mothers aged 35 years or more who gave birth for the first time increased from 8.1 to 11.1 per cent over the five year period, and the proportion of multiparous mothers increased from 19.5 to 22.5 per cent.

\section{FIGURE1}

CONFINEMENTS AMONG MOTHERS AGED LESS THAN 20 YEARS AND 35 YEARS AND OVER, NSW 1996-2000

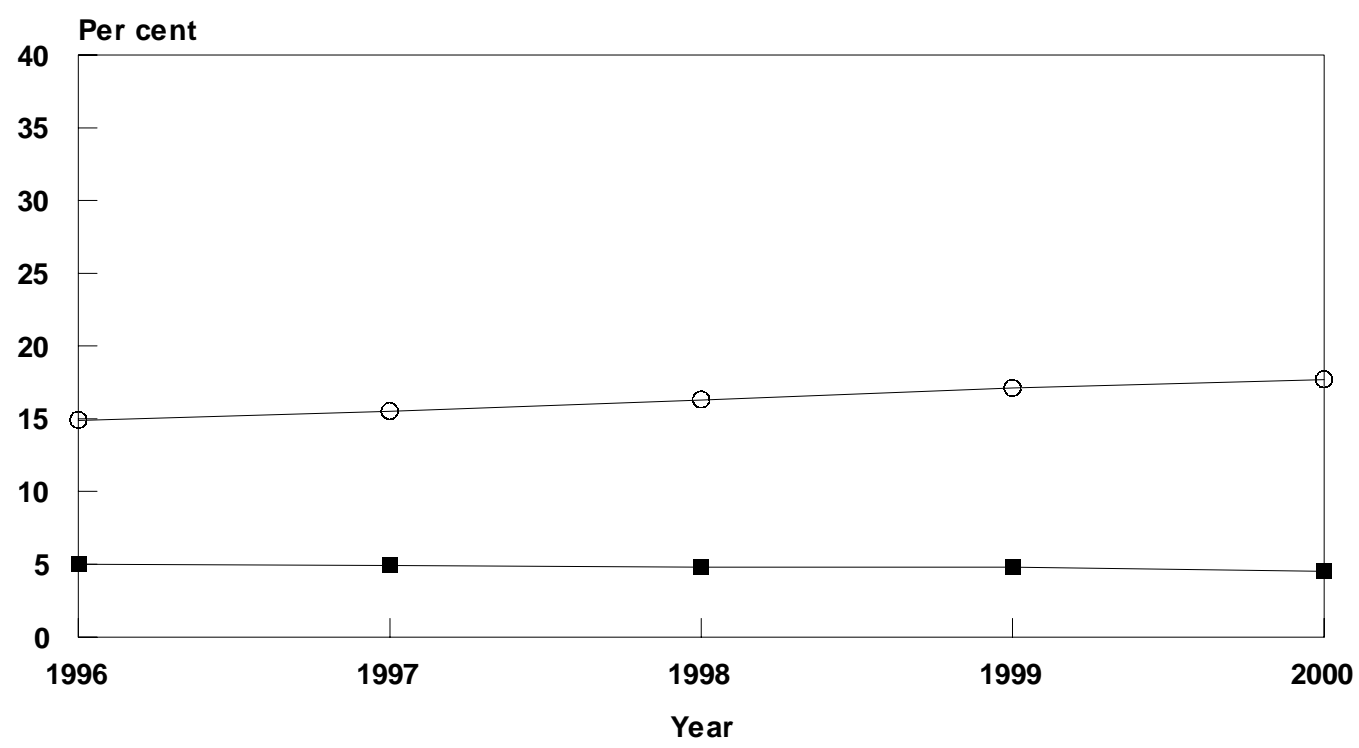

- Less than 20 years $\theta 35$ years and over

Source: NSW Midwives Data Collection Epidemiology and Surveillance Branch, NSW Department of Health.

\section{TABLE 3}

CONFINEMENTS BY MATERNAL AGE, NSW 1996-2000

\begin{tabular}{|c|c|c|c|c|c|c|c|c|c|c|}
\hline \multirow[t]{2}{*}{$\begin{array}{l}\text { Maternal age } \\
\text { (years) }\end{array}$} & \multicolumn{2}{|c|}{1996} & \multicolumn{4}{|c|}{$\begin{array}{c}\text { Year } \\
1998\end{array}$} & \multicolumn{2}{|c|}{1999} & \multicolumn{2}{|c|}{2000} \\
\hline & No. & $\%$ & No. & $\%$ & No. & $\%$ & No. & $\%$ & No. & $\%$ \\
\hline $12-19$ & 4295 & 5.0 & 4291 & 4.9 & 4118 & 4.8 & 4099 & 4.8 & 3853 & 4.5 \\
\hline 20-34 & 68239 & 80.0 & 69114 & 79.5 & 67034 & 78.8 & 67171 & 78.1 & 67249 & 77.8 \\
\hline $35+$ & 12712 & 14.9 & 13465 & 15.5 & 13839 & 16.3 & 14668 & 17.1 & 15334 & 17.7 \\
\hline Not stated & 56 & 0.1 & 50 & 0.1 & 81 & 0.1 & 29 & 0.0 & 24 & 0.0 \\
\hline TOTAL & 85302 & 100.0 & 86920 & 100.0 & 85072 & 100.0 & 85967 & 100.0 & 86460 & 100.0 \\
\hline
\end{tabular}

Source: NSW Midwives Data Collection (HOIST). Epidemiology and Surveillance Branch, NSW Department of Health. 


\subsection{MATERNAL COUNTRY OF BIRTH}

In the period 1996-2000, almost three-quarters of confinements were to mothers who were born in Australia. Numbers of confinements to mothers born in Southern European countries continued to decline while confinements to mothers born in Pacific Island countries increased over the five year period (Table 4). For Asian countries, the number of confinements increased primarily among mothers born in China, Vietnam, North Korea and South Korea and decreased for mothers born in Hong Kong. Further information on maternal country of birth is shown in Part 4.

\section{TABLE 4}

CONFINEMENTS BY MATERNAL COUNTRY OF BIRTH, NSW 1996-2000\#

\begin{tabular}{|c|c|c|c|c|c|c|c|c|c|c|}
\hline \multirow[t]{2}{*}{ Country of birth } & \multicolumn{4}{|c|}{1996} & \multicolumn{2}{|c|}{ Year } & \multicolumn{2}{|c|}{1999} & \multicolumn{2}{|c|}{2000} \\
\hline & No. & $\%$ & No. & $\%$ & No. & $\%$ & No. & $\%$ & No. & $\%$ \\
\hline Australia & 62309 & 73.0 & 63292 & 72.8 & 62606 & 73.6 & 62555 & 72.8 & 62368 & 72.1 \\
\hline United Kingdom & 2707 & 3.2 & 2593 & 3.0 & 2471 & 2.9 & 2627 & 3.1 & 2557 & 3.0 \\
\hline China & 1950 & 2.3 & 2111 & 2.4 & 1892 & 2.2 & 2015 & 2.3 & 2163 & 2.5 \\
\hline Vietnam & 1865 & 2.2 & 1853 & 2.1 & 1462 & 1.7 & 1804 & 2.1 & 2053 & 2.4 \\
\hline New Zealand & 1790 & 2.1 & 1826 & 2.1 & 1762 & 2.1 & 1966 & 2.3 & 1962 & 2.3 \\
\hline Lebanon & 2042 & 2.4 & 1983 & 2.3 & 1942 & 2.3 & 1788 & 2.1 & 1766 & 2.0 \\
\hline Philippines & 1266 & 1.5 & 1275 & 1.5 & 1308 & 1.5 & 1319 & 1.5 & 1315 & 1.5 \\
\hline Fiji & 602 & 0.7 & 603 & 0.7 & 640 & 0.8 & 604 & 0.7 & 688 & 0.8 \\
\hline India & 576 & 0.7 & 673 & 0.8 & 634 & 0.7 & 635 & 0.7 & 643 & 0.7 \\
\hline Former Yugoslavia & 689 & 0.8 & 714 & 0.8 & 659 & 0.8 & 662 & 0.8 & 627 & 0.7 \\
\hline Indonesia & 381 & 0.4 & 398 & 0.5 & 424 & 0.5 & 460 & 0.5 & 566 & 0.7 \\
\hline Iraq & 234 & 0.3 & 326 & 0.4 & 360 & 0.4 & 414 & 0.5 & 455 & 0.5 \\
\hline South Korea & 261 & 0.3 & 308 & 0.4 & 370 & 0.4 & 370 & 0.4 & 426 & 0.5 \\
\hline South Africa & 310 & 0.4 & 349 & 0.4 & 329 & 0.4 & 386 & 0.4 & 387 & 0.4 \\
\hline United States of America & 322 & 0.4 & 330 & 0.4 & 340 & 0.4 & 372 & 0.4 & 377 & 0.4 \\
\hline Hong Kong & 659 & 0.8 & 531 & 0.6 & 433 & 0.5 & 409 & 0.5 & 357 & 0.4 \\
\hline Turkey & 376 & 0.4 & 364 & 0.4 & 340 & 0.4 & 314 & 0.4 & 335 & 0.4 \\
\hline Cambodia & 311 & 0.4 & 305 & 0.4 & 238 & 0.3 & 303 & 0.4 & 326 & 0.4 \\
\hline Western Samoa & 280 & 0.3 & 312 & 0.4 & 349 & 0.4 & 318 & 0.4 & 320 & 0.4 \\
\hline Malaysia & 308 & 0.4 & 307 & 0.4 & 259 & 0.3 & 286 & 0.3 & 319 & 0.4 \\
\hline Sri Lanka & 274 & 0.3 & 279 & 0.3 & 276 & 0.3 & 295 & 0.3 & 304 & 0.4 \\
\hline Tonga & 263 & 0.3 & 292 & 0.3 & 312 & 0.4 & 308 & 0.4 & 296 & 0.3 \\
\hline Ireland & 276 & 0.3 & 275 & 0.3 & 280 & 0.3 & 287 & 0.3 & 273 & 0.3 \\
\hline Japan & 215 & 0.3 & 226 & 0.3 & 239 & 0.3 & 264 & 0.3 & 252 & 0.3 \\
\hline Pakistan & 137 & 0.2 & 202 & 0.2 & 200 & 0.2 & 192 & 0.2 & 224 & 0.3 \\
\hline Germany & 208 & 0.2 & 213 & 0.2 & 187 & 0.2 & 226 & 0.3 & 204 & 0.2 \\
\hline Chile & 220 & 0.3 & 209 & 0.2 & 214 & 0.3 & 224 & 0.3 & 202 & 0.2 \\
\hline Thailand & 166 & 0.2 & 186 & 0.2 & 194 & 0.2 & 207 & 0.2 & 199 & 0.2 \\
\hline Egypt & 254 & 0.3 & 253 & 0.3 & 202 & 0.2 & 218 & 0.3 & 196 & 0.2 \\
\hline Italy & 285 & 0.3 & 272 & 0.3 & 230 & 0.3 & 221 & 0.3 & 191 & 0.2 \\
\hline Bangladesh & 94 & 0.1 & 125 & 0.1 & 140 & 0.2 & 134 & 0.2 & 179 & 0.2 \\
\hline Canada & 170 & 0.2 & 155 & 0.2 & 177 & 0.2 & 185 & 0.2 & 177 & 0.2 \\
\hline Iran & 134 & 0.2 & 147 & 0.2 & 139 & 0.2 & 140 & 0.2 & 153 & 0.2 \\
\hline North Korea & 59 & 0.1 & 53 & 0.1 & 71 & 0.1 & 90 & 0.1 & 140 & 0.2 \\
\hline Syria & 154 & 0.2 & 141 & 0.2 & 143 & 0.2 & 145 & 0.2 & 138 & 0.2 \\
\hline Laos & 140 & 0.2 & 133 & 0.2 & 126 & 0.1 & 118 & 0.1 & 136 & 0.2 \\
\hline Papua New Guinea & 130 & 0.2 & 150 & 0.2 & 131 & 0.2 & 136 & 0.2 & 132 & 0.2 \\
\hline Greece & 174 & 0.2 & 178 & 0.2 & 140 & 0.2 & 109 & 0.1 & 119 & 0.1 \\
\hline France & 93 & 0.1 & 85 & 0.1 & 114 & 0.1 & 108 & 0.1 & 113 & 0.1 \\
\hline Uruguay & 105 & 0.1 & 102 & 0.1 & 123 & 0.1 & 91 & 0.1 & 111 & 0.1 \\
\hline Netherlands & 84 & 0.1 & 94 & 0.1 & 92 & 0.1 & 102 & 0.1 & 109 & 0.1 \\
\hline Poland & 125 & 0.1 & 120 & 0.1 & 100 & 0.1 & 123 & 0.1 & 104 & 0.1 \\
\hline Singapore & 109 & 0.1 & 89 & 0.1 & 102 & 0.1 & 101 & 0.1 & 104 & 0.1 \\
\hline Portugal & 145 & 0.2 & 121 & 0.1 & 136 & 0.2 & 120 & 0.1 & 101 & 0.1 \\
\hline Taiwan & 75 & 0.1 & 89 & 0.1 & 85 & 0.1 & 73 & 0.1 & 101 & 0.1 \\
\hline Cook Islands & 59 & 0.1 & 107 & 0.1 & 86 & 0.1 & 92 & 0.1 & 100 & 0.1 \\
\hline Other-Not stated & 1916 & 2.2 & 2171 & 2.5 & 2015 & 2.4 & 2051 & 2.4 & 2092 & 2.4 \\
\hline TOTAL & 85302 & 100.0 & 86920 & 100.0 & 85072 & 100.0 & 85967 & 100.0 & 86460 & 100.0 \\
\hline
\end{tabular}

Source: NSW Midwives Data Collection (HOIST). Epidemiology and Surveillance Branch, NSW Department of Health.

\# $\quad$ Countries of birth for which there were 100 or more confinements in 2000. 


\subsection{MATERNAL ABORIGINALITY}

The reported number of Aboriginal or Torres Strait Islander mothers giving birth increased from 1,712 in 1996 (2.0 per cent of all mothers) to 2,105 in 2000 (2.4 per cent of all mothers) (Table 5). Part of this increase is likely to be due to an increased willingness of mothers to be identified as Aboriginal or Torres Strait Islander. Further information on maternal Aboriginality and reporting of Aborginality is shown in Part 3.

\section{TABLE 5}

CONFINEMENTS BY MATERNAL ABORIGINALITY, NSW 1996-2000

\begin{tabular}{|c|c|c|c|c|c|c|c|c|c|c|}
\hline \multirow[t]{2}{*}{ Aboriginality } & \multicolumn{2}{|c|}{1996} & \multicolumn{2}{|c|}{1997} & \multicolumn{2}{|c|}{$\begin{array}{c}\text { Year } \\
1998\end{array}$} & \multicolumn{2}{|c|}{1999} & \multicolumn{2}{|c|}{2000} \\
\hline & No. & $\%$ & No. & $\%$ & No. & $\%$ & No. & $\%$ & No. & $\%$ \\
\hline \multicolumn{11}{|l|}{ Aboriginal or Torres } \\
\hline Strait Islander & 1712 & 2.0 & 1842 & 2.1 & 2043 & 2.4 & 2059 & 2.4 & 2105 & 2.4 \\
\hline Non-Aboriginal or & & & & & & & & & & \\
\hline Torres Strait Islander & 83486 & 97.9 & 84854 & 97.6 & 82787 & 97.3 & 83899 & 97.6 & 84306 & 97.5 \\
\hline Not stated & 104 & 0.1 & 224 & 0.3 & 242 & 0.3 & 9 & 0.0 & 49 & 0.1 \\
\hline TOTAL & 85302 & 100.0 & 86920 & 100.0 & 85072 & 100.0 & 85967 & 100.0 & 86460 & 100.0 \\
\hline
\end{tabular}

Source: NSW Midwives Data Collection (HOIST). Epidemiology and Surveillance Branch, NSW Department of Health.

\subsection{NUMBER OF PREVIOUS PREGNANCIES}

In recent years there were no substantial changes in the reported number of previous pregnancies greater than 20 weeks gestation (Table 6). About 41 per cent of mothers gave birth for the first time, about 57 per cent gave birth to a second to fourth baby and less than 2 per cent reported more than five previous births.

\section{TABLE 6}

CONFINEMENTS BY NUMBER OF PREVIOUS PREGNANCIES, NSW 1996-2000

\begin{tabular}{|c|c|c|c|c|c|c|c|c|c|c|}
\hline \multirow{2}{*}{$\begin{array}{l}\text { Number of previous } \\
\text { pregnancies } \\
\text { ( }>20 \text { weeks gestation) }\end{array}$} & \multicolumn{4}{|c|}{1996} & \multicolumn{2}{|c|}{$\begin{array}{l}\text { Year } \\
1998\end{array}$} & \multicolumn{2}{|c|}{1999} & \multicolumn{2}{|c|}{2000} \\
\hline & No. & $\%$ & No. & $\%$ & No. & $\%$ & No. & $\%$ & No. & $\%$ \\
\hline 0 & 34267 & 40.2 & 34984 & 40.2 & 34376 & 40.4 & 35311 & 41.1 & 35953 & 41.6 \\
\hline $1-4$ & 49692 & 58.3 & 50451 & 58.0 & 49462 & 58.1 & 49432 & 57.5 & 49146 & 56.8 \\
\hline $5+$ & 1237 & 1.5 & 1267 & 1.5 & 1184 & 1.4 & 1206 & 1.4 & 1331 & 1.5 \\
\hline Not stated & 106 & 0.1 & 218 & 0.3 & 50 & 0.1 & 18 & 0.0 & 30 & 0.0 \\
\hline TOTAL & 85302 & 100.0 & 86920 & 100.0 & 85072 & 100.0 & 85967 & 100.0 & 86460 & 100.0 \\
\hline
\end{tabular}

Source: NSW Midwives Data Collection (HOIST). Epidemiology and Surveillance Branch, NSW Department of Health. 


\subsection{DURATION OF PREGNANCY AT FIRST ANTENATAL VISIT}

Since 1996, the proportion of mothers starting antenatal care at 20-plus weeks gestation has been stable at 12-13 per cent (Table 7).

\begin{tabular}{|c|c|c|c|c|c|c|c|c|c|c|}
\hline \multirow[t]{2}{*}{$\begin{array}{l}\text { Duration of } \\
\text { pregnancy (weeks) }\end{array}$} & \multicolumn{2}{|c|}{1996} & \multicolumn{2}{|c|}{1997} & \multicolumn{2}{|c|}{$\begin{array}{l}\text { Year } \\
1998\end{array}$} & \multicolumn{2}{|c|}{1999} & \multicolumn{2}{|c|}{2000} \\
\hline & No. & $\%$ & No. & $\%$ & No. & $\%$ & No. & $\%$ & No. & $\%$ \\
\hline $0-19$ & 72726 & 85.3 & 73666 & 84.8 & 72257 & 84.9 & 74077 & 86.2 & 74803 & 86.5 \\
\hline 20-plus & 10972 & 12.9 & 11549 & 13.3 & 11410 & 13.4 & 10979 & 12.8 & 10748 & 12.4 \\
\hline Not stated & 1604 & 1.9 & 1705 & 2.0 & 1405 & 1.7 & 911 & 1.1 & 909 & 1.1 \\
\hline TOTAL & 85302 & 100.0 & 86920 & 100.0 & 85072 & 100.0 & 85967 & 100.0 & 86460 & 100.0 \\
\hline
\end{tabular}

Source: NSW Midwives Data Collection (HOIST). Epidemiology and Surveillance Branch, NSW Department of Health.

\subsection{SMOKING IN PREGNANCY}

The proportion of mothers reporting any smoking during pregnancy declined between 1996 and 2000: in 1996, 17,957 (21.1 per cent) mothers reported smoking in pregnancy, compared to, 17,871 (20.6 per cent) in 1997, 16,859 (19.8 per cent) in 1998, 16,302 (19.0 per cent) in 1999, and 15,001 (17.4 per cent) in 2000 .
Of mothers who smoked during pregnancy in 2000, about four per cent stopped smoking before the second half of pregnancy.

Over the five year period, among those who smoked in the second half of pregnancy, there was a trend towards smoking fewer cigarettes per day (Table 8).

\section{TABLE 8}

MOTHERS WHO SMOKED AT ALL DURING PREGNANCY BY NUMBER OF CIGARETTES SMOKED IN THE SECOND HALF OF PREGNANCY, NSW 1996-2000

\begin{tabular}{|c|c|c|c|c|c|c|c|c|c|c|}
\hline \multirow[t]{2}{*}{$\begin{array}{l}\text { Cigarettes smoked in the } \\
\text { second half of pregnancy }\end{array}$} & \multicolumn{2}{|c|}{1996} & \multicolumn{2}{|c|}{1997} & \multicolumn{2}{|c|}{$\begin{array}{l}\text { Year } \\
1998\end{array}$} & \multicolumn{2}{|c|}{1999} & \multicolumn{2}{|c|}{2000} \\
\hline & No. & $\%$ & No. & $\%$ & No. & $\%$ & No. & $\%$ & No. & $\%$ \\
\hline None & 556 & 3.1 & 570 & 3.2 & 690 & 4.1 & 739 & 4.5 & 622 & 4.1 \\
\hline More than ten per day & 8842 & 49.2 & 8574 & 48.0 & 8171 & 48.5 & 7966 & 48.9 & 7005 & 46.7 \\
\hline $1-10$ per day & 7925 & 44.1 & 7872 & 44.0 & 7634 & 45.3 & 7303 & 44.8 & 7092 & 47.3 \\
\hline Smoked, amount not stated & 623 & 3.5 & 833 & 4.7 & 358 & 2.1 & 294 & 1.8 & 282 & 1.9 \\
\hline Not stated & 11 & 0.1 & 22 & 0.1 & 6 & 0.0 & 0 & 0.0 & 0 & 0.0 \\
\hline TOTAL & 17957 & 100.0 & 17871 & 100.0 & 16859 & 100.0 & 16302 & 100.0 & 15001 & 100.0 \\
\hline
\end{tabular}

Source: NSW Midwives Data Collection (HOIST). Epidemiology and Surveillance Branch, NSW Department of Health. 


\subsection{PLACE OF BIRTH}

In 2000, the majority of mothers planned to give birth in a hospital labour ward, and about four per cent of mothers planned to give birth in a birth centre (Table 9, Figure 2). About one third of mothers who planned to give birth in a birth centre actually did so.
Over the five year period 1996-2000, there was a slight increase in the numbers of births in hospital and a decline in the numbers of births reported in birth centres and at home.

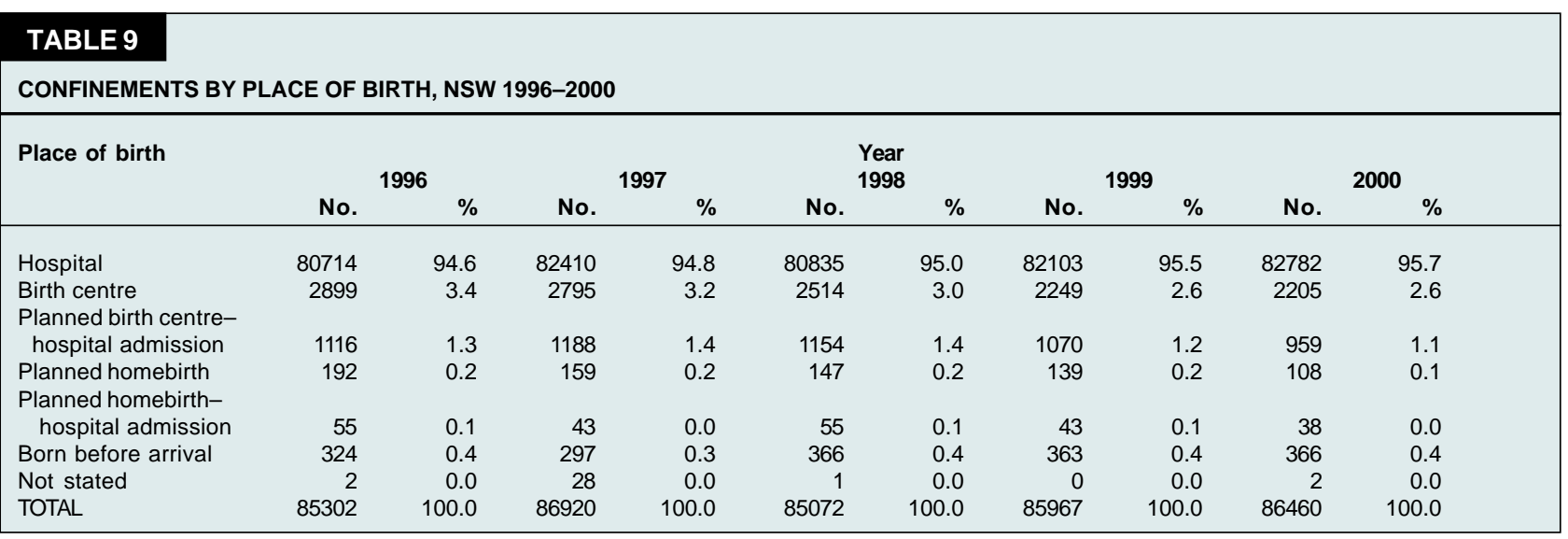

Source: NSW Midwives Data Collection (HOIST). Epidemiology and Surveillance Branch, NSW Department of Health.

\section{FIGURE2}

CONFINEMENTS BY PLANNED PLACE OF BIRTH, NSW 1996-2000

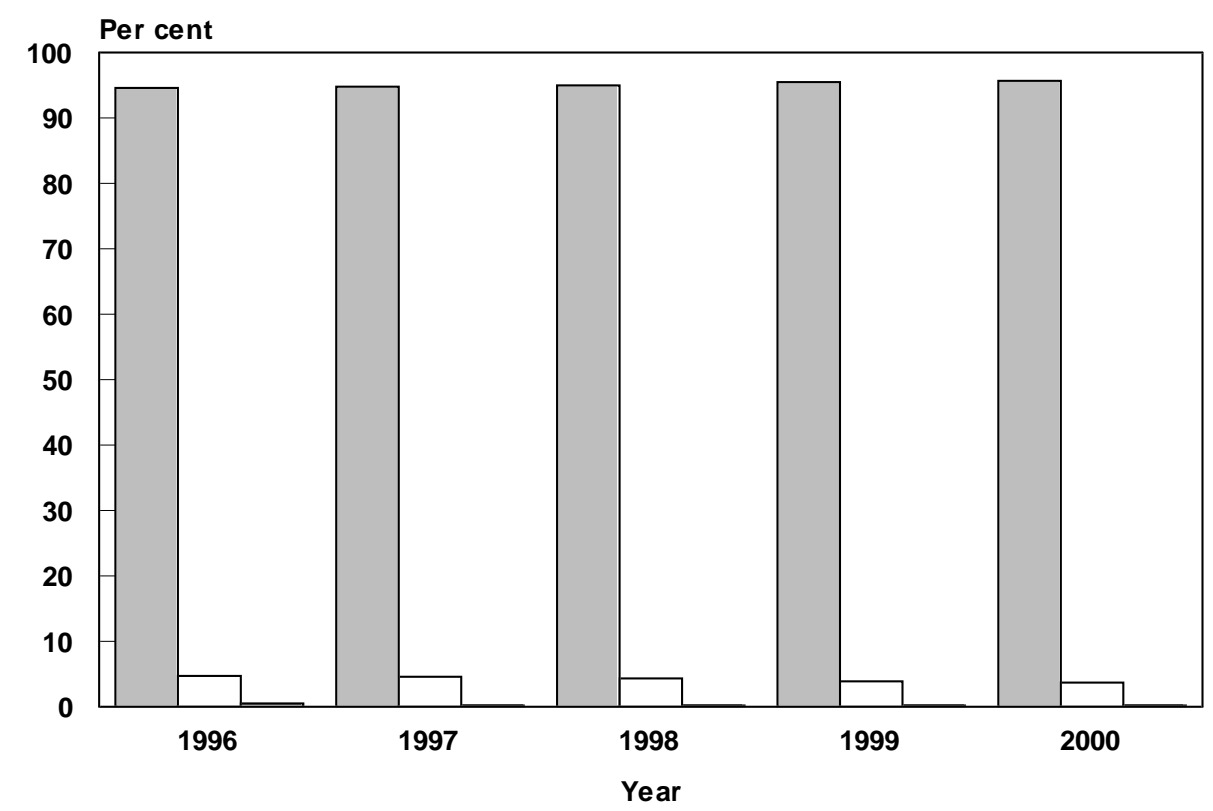

$\square$ Hospital $\square$ Birth centre $\square$ Home

Source: NSW Midwives Data Collection (HOIST). Epidemiology and Surveillance Branch, NSW Department of Health. 


\subsection{LABOUR AND DELIVERY}

The rate of induction of labour rose slightly from 20.5 per cent in 1996 to 23.6 per cent in 2000. About one in 10 labours were augmented with oxytocics or prostaglandins in 2000. The rate of spontaneous onset of labour fell from 70.0 to 64.9 per cent (Table 10 ).

The rate of normal vaginal birth decreased from 70.7 per cent in 1996 to 67.1 per cent in 2000 (Table 11). The caesarean section rate increased from 17.6 to 21.3 per cent.
The rate of instrumental delivery remained steady at about 10 to 11 per cent, accompanied by a change in the pattern of instrumental delivery: the rate of vacuum extraction rose from 3.9 to 6.2 per cent and the rate of forceps delivery declined from 6.7 to 4.5 per cent.

While operative and instrumental deliveries continue to be more common among privately than publicly insured mothers, the changing pattern in type of delivery is evident in both groups (Table 12).

\section{TABLE 10}

CONFINEMENTS BY ONSET AND AUGMENTATION OF LABOUR, NSW 1996-2000

\begin{tabular}{|c|c|c|c|c|c|c|c|c|c|c|}
\hline \multirow[t]{2}{*}{ Onset of labour } & \multicolumn{2}{|c|}{1996} & \multicolumn{2}{|c|}{1997} & \multicolumn{2}{|c|}{ Year } & \multicolumn{2}{|c|}{1999} & \multicolumn{2}{|c|}{2000} \\
\hline & No. & $\%$ & No. & $\%$ & No. & $\%$ & No. & $\%$ & No. & $\%$ \\
\hline Spontaneous & 39891 & 46.8 & 39839 & 45.8 & 39281 & 46.2 & 39706 & 46.2 & 40042 & 46.3 \\
\hline Spontaneous & & & & & & & & & & \\
\hline augmented with ARM & 10564 & 12.4 & 9764 & 11.2 & 7997 & 9.4 & 7844 & 9.1 & 7014 & 8.1 \\
\hline $\begin{array}{l}\text { Spontaneous } \\
\text { augmented with } \\
\text { oxytocics- }\end{array}$ & & & & & & & & & & \\
\hline prostaglandins & 9222 & 10.8 & 9622 & 11.1 & 8411 & 9.9 & 8657 & 10.1 & 9050 & 10.5 \\
\hline No labour & 8052 & 9.4 & 8616 & 9.9 & 8800 & 10.3 & 9147 & 10.6 & 9926 & 11.5 \\
\hline $\begin{array}{l}\text { Induced- } \\
\text { oxytocics- }\end{array}$ & & & & & & & & & & \\
\hline prostaglandins & 5644 & 6.6 & 5934 & 6.8 & 7893 & 9.3 & 7626 & 8.9 & 7493 & 8.7 \\
\hline Induced- ARM only & 1211 & 1.4 & 1238 & 1.4 & 1462 & 1.7 & 1305 & 1.5 & 1196 & 1.4 \\
\hline Induced- & & & & & & & & & & \\
\hline $\begin{array}{l}\text { ARM+oxytocics- } \\
\text { prostaglandins }\end{array}$ & 10601 & 12.4 & 11722 & 13.5 & 11069 & 13.0 & 11527 & 13.4 & 11516 & 13.3 \\
\hline Induced- othe $\mathrm{r}^{\mathrm{r \#}}$ & 67 & 0.1 & 87 & 0.1 & 138 & 0.2 & 154 & 0.2 & 215 & 0.2 \\
\hline Not stated & 50 & 0.1 & 98 & 0.1 & 21 & 0.0 & 1 & 0.0 & 8 & 0.0 \\
\hline TOTAL & 85302 & 100.0 & 86920 & 100.0 & 85072 & 100.0 & 85967 & 100.0 & 86460 & 100.0 \\
\hline
\end{tabular}

Source: NSW Midwives Data Collection (HOIST). Epidemiology and Surveillance Branch, NSW Department of Health

This category includes other forms of induction such at Foley's catheter.

\section{TABLE 11}

CONFINEMENTS BY TYPE OF DELIVERY, NSW 1996-2000

\begin{tabular}{|c|c|c|c|c|c|c|c|c|c|c|}
\hline \multirow[t]{2}{*}{ Type of delivery } & \multicolumn{2}{|c|}{1996} & \multicolumn{2}{|c|}{1997} & \multicolumn{2}{|c|}{$\begin{array}{l}\text { Year } \\
1998\end{array}$} & \multicolumn{2}{|c|}{1999} & \multicolumn{2}{|c|}{2000} \\
\hline & No. & $\%$ & No. & $\%$ & No. & $\%$ & No. & $\%$ & No. & $\%$ \\
\hline Normal vaginal & 60339 & 70.7 & 61175 & 70.4 & 59097 & 69.5 & 58951 & 68.6 & 58049 & 67.1 \\
\hline Forceps & 5724 & 6.7 & 5014 & 5.8 & 4478 & 5.3 & 4190 & 4.9 & 3904 & 4.5 \\
\hline Vacuum extraction & 3286 & 3.9 & 3919 & 4.5 & 4453 & 5.2 & 5152 & 6.0 & 5367 & 6.2 \\
\hline Vaginal breech & 874 & 1.0 & 921 & 1.1 & 805 & 0.9 & 762 & 0.9 & 669 & 0.8 \\
\hline $\begin{array}{l}\text { Elective caesarean } \\
\text { section } \\
\text { Emergency }\end{array}$ & 8052 & 9.4 & 8616 & 9.9 & 8800 & 10.3 & 9147 & 10.6 & 9926 & 11.5 \\
\hline caesarean section\# & 6955 & 8.2 & 7195 & 8.3 & 7416 & 8.7 & 7765 & 9.0 & 8530 & 9.9 \\
\hline Not stated & 72 & 0.1 & 80 & 0.1 & 23 & 0.0 & 0 & 0.0 & 15 & 0.0 \\
\hline TOTAL & 85302 & 100.0 & 86920 & 100.0 & 85072 & 100.0 & 85967 & 100.0 & 86460 & 100.0 \\
\hline
\end{tabular}

Source: NSW Midwives Data Collection (HOIST). Epidemiology and Surveillance Branch, NSW Department of Health.

\# Emergency caesarean section includes caesarean sections where the onset of labour was not stated. 


\section{TABLE 12}

CONFINEMENTS BY HEALTH INSURANCE STATUS AND TYPE OF DELIVERY, NSW 1995-1999

\begin{tabular}{|c|c|c|c|c|c|c|c|c|c|c|}
\hline \multirow[t]{2}{*}{$\begin{array}{l}\text { Insurance status- } \\
\text { type of delivery }\end{array}$} & \multicolumn{8}{|c|}{ Year } & \multicolumn{2}{|c|}{1999} \\
\hline & No. & $\%$ & No. & $\%$ & No. & $\%$ & No. & $\%$ & No. & $\%$ \\
\hline \multicolumn{11}{|l|}{ Public } \\
\hline Normal vaginal & 42004 & 75.7 & 43495 & 75.4 & 45183 & 75.1 & 42967 & 73.8 & 44151 & 72.8 \\
\hline Forceps & 2970 & 5.4 & 3019 & 5.2 & 2643 & 4.4 & 2268 & 3.9 & 2385 & 3.9 \\
\hline Vacuum extraction & 1528 & 2.8 & 1587 & 2.8 & 2295 & 3.8 & 2593 & 4.5 & 3157 & 5.2 \\
\hline Vaginal breech & 630 & 1.1 & 680 & 1.2 & 733 & 1.2 & 611 & 1.0 & 596 & 1.0 \\
\hline Elective caesarean section & 4184 & 7.5 & 4408 & 7.6 & 4684 & 7.8 & 4890 & 8.4 & 5193 & 8.6 \\
\hline Emergency caesarean section" & 4133 & 7.4 & 4472 & 7.7 & 4610 & 7.7 & 4870 & 8.4 & 5198 & 8.6 \\
\hline Not stated & 36 & 0.1 & 47 & 0.1 & 45 & 0.1 & 13 & 0.0 & 0 & 0.0 \\
\hline TOTAL & 55485 & 100.0 & 57708 & 100.0 & 60193 & 100.0 & 58212 & 100.0 & 60680 & 100.0 \\
\hline \multicolumn{11}{|l|}{ Private } \\
\hline Normal vaginal & 19194 & 62.4 & 16834 & 61.0 & 15929 & 59.8 & 15325 & 59.4 & 14206 & 58.1 \\
\hline Forceps & 3112 & 10.1 & 2705 & 9.8 & 2368 & 8.9 & 2167 & 8.4 & 1780 & 7.3 \\
\hline Vacuum extraction & 1415 & 4.6 & 1697 & 6.2 & 1621 & 6.1 & 1818 & 7.0 & 1969 & 8.0 \\
\hline Vaginal breech & 288 & 0.9 & 194 & 0.7 & 185 & 0.7 & 162 & 0.6 & 139 & 0.6 \\
\hline Elective caesarean section & 4046 & 13.2 & 3642 & 13.2 & 3927 & 14.7 & 3835 & 14.9 & 3859 & 15.8 \\
\hline Emergency caesarean section\# & 2685 & 8.7 & 2482 & 9.0 & 2581 & 9.7 & 2482 & 9.6 & 2508 & 10.3 \\
\hline Not stated & 17 & 0.1 & 25 & 0.1 & 34 & 0.1 & 10 & 0.0 & 0 & 0.0 \\
\hline TOTAL & 30757 & 100.0 & 27579 & 100.0 & 26645 & 100.0 & 25799 & 100.0 & 24461 & 100.0 \\
\hline \multicolumn{11}{|l|}{ TOTAL\# } \\
\hline Normal vaginal & 61215 & 71.0 & 60339 & 70.7 & 61175 & 70.4 & 59097 & 69.5 & 58951 & 68.6 \\
\hline Forceps & 6083 & 7.1 & 5724 & 6.7 & 5014 & 5.8 & 4478 & 5.3 & 4190 & 4.9 \\
\hline Vacuum extraction & 2943 & 3.4 & 3286 & 3.9 & 3919 & 4.5 & 4453 & 5.2 & 5152 & 6.0 \\
\hline Vaginal breech & 918 & 1.1 & 874 & 1.0 & 921 & 1.1 & 805 & 0.9 & 762 & 0.9 \\
\hline Elective caesarean section8231 & 9.5 & 8052 & 9.4 & 8616 & 9.9 & 8800 & 10.3 & 9147 & 10.6 & \\
\hline Emergency caesarean section\# & 6818 & 7.9 & 6955 & 8.2 & 7195 & 8.3 & 7416 & 8.7 & 7765 & 9.0 \\
\hline Not stated & 55 & 0.1 & 72 & 0.1 & 80 & 0.1 & 23 & 0.0 & 0 & 0.0 \\
\hline TOTAL & 86263 & 100.0 & 85302 & 100.0 & 86920 & 100.0 & 85072 & 100.0 & 85967 & 100.0 \\
\hline
\end{tabular}

Source: 1995-1997: NSW Midwives Data Collection (HOIST). 1998-1999: Linked data of the NSW Midwives Data Collection and NSW Inpatient Statistics Collection. Epidemiology and Surveillance Branch, NSW Department of Health

\# Emergency caesarean section includes caesarean sections where the onset of labour was not stated.

\#\# Total includes confinements where type of health insurance was not stated.

\subsection{PAIN RELIEF}

Information on pain relief was collected by the MDC from 1998. Over the three years 1998 to 2000 there was a trend towards increased use of epidural and spinal anaesthetics.

In 2000, almost one half (48.9 per cent) of all mothers used nitrous oxide for pain relief, 29.8 per cent had an epidural anaesthetic, 26.2 per cent received intra-muscular narcotics, and no pain relief was reported for 12.2 per cent of mothers (Table 13).

\section{TABLE 13}

CONFINEMENTS BY TYPE OF PAIN RELIEF, NSW 1998-2000

\begin{tabular}{|c|c|c|c|c|c|c|}
\hline \multirow[t]{2}{*}{ Type of pain relief\# } & \multicolumn{6}{|c|}{ Year } \\
\hline & No. & $\%$ & No. & $\%$ & No. & $\%$ \\
\hline Epidural & 22917 & 26.9 & 24289 & 28.3 & 25728 & 29.8 \\
\hline General anaesthetic & 5004 & 5.9 & 4735 & 5.5 & 4753 & 5.5 \\
\hline IM Narcotics & 22274 & 26.2 & 22800 & 26.5 & 22654 & 26.2 \\
\hline Nitrous Oxide & 41273 & 48.5 & 42361 & 49.3 & 42303 & 48.9 \\
\hline Spinal & 3314 & 3.9 & 4179 & 4.9 & 5248 & 6.1 \\
\hline Nil & 12656 & 14.9 & 11468 & 13.3 & 10518 & 12.2 \\
\hline TOTAL CONFINEMENTS & 85072 & 100.0 & 85967 & 100.0 & 86460 & 100.0 \\
\hline
\end{tabular}

Source: NSW Midwives Data Collection (HOIST). Epidemiology and Surveillance Branch, NSW Department of Health.

\# $\quad$ More than one type of pain relief may be used. 


\subsection{BABY SEX}

There were no signification changes in the pattern of baby sex since 1996, with slightly more male babies born than females in each year. In 2000, 45,346 (51.6 per cent) of babies were male, 42,539 (48.4 per cent) were female, 15 were of indeterminate sex, and the sex was not reported for 22 babies. This compares with babies born in 1996, when 44,520 (51.5 per cent) were male, 41,841 (48.4 per cent) were female, 18 were of indeterminate sex, and the sex was not reported for 50 babies.

\subsection{GESTATIONAL AGE}

Since 1996 there has been a slight increase in the percentage of babies born at 32-36 weeks gestation, and a decrease in the percentage born at 42 weeks or more (Table 14). The overall rate of premature births (less than 37 weeks gestation) rose from 6.7 per cent in 1996 to 7.3 per cent in 2000. There was no substantial change in the proportion of babies who were very premature (less than 32 weeks) or extremely premature (less than 28 weeks).

\section{TABLE 14}

BIRTHS BY GESTATIONAL AGE, NSW 1996-2000

\begin{tabular}{|c|c|c|c|c|c|c|c|c|c|c|}
\hline \multirow[t]{2}{*}{$\begin{array}{l}\text { Gestational age } \\
\text { (weeks) }\end{array}$} & \multicolumn{4}{|c|}{1996} & \multicolumn{2}{|c|}{$\begin{array}{c}\text { Year } \\
1998\end{array}$} & \multicolumn{2}{|c|}{1999} & \multicolumn{2}{|c|}{2000} \\
\hline & No. & $\%$ & No. & $\%$ & No. & $\%$ & No. & $\%$ & No. & $\%$ \\
\hline $20-27$ & 531 & 0.6 & 562 & 0.6 & 588 & 0.7 & 585 & 0.7 & 623 & 0.7 \\
\hline $28-31$ & 574 & 0.7 & 596 & 0.7 & 607 & 0.7 & 625 & 0.7 & 663 & 0.8 \\
\hline $32-36$ & 4689 & 5.4 & 4852 & 5.5 & 4758 & 5.5 & 5026 & 5.8 & 5114 & 5.8 \\
\hline $37-41$ & 78406 & 90.7 & 79987 & 90.8 & 78463 & 90.9 & 79114 & 90.6 & 79368 & 90.3 \\
\hline $42+$ & 2187 & 2.5 & 2091 & 2.4 & 1871 & 2.2 & 1932 & 2.2 & 2148 & 2.4 \\
\hline Not stated & 42 & 0.0 & 45 & 0.1 & 18 & 0.0 & 7 & 0.0 & 6 & 0.0 \\
\hline TOTAL & 86429 & 100.0 & 88133 & 100.0 & 86305 & 100.0 & 87289 & 100.0 & 87922 & 100.0 \\
\hline
\end{tabular}

Source: NSW Midwives Data Collection (HOIST). Epidemiology and Surveillance Branch, NSW Department of Health.

\subsection{BIRTHWEIGHT}

Since 1996, the rate of low birthweight (less than 2,500 grams) was constant at about six per cent (Table 15). The rate was 6.4 per cent in 2000 .

\section{TABLE 15}

BIRTHS BY BIRTHWEIGHT, NSW 1996-2000

\begin{tabular}{|c|c|c|c|c|c|c|c|c|c|c|}
\hline \multirow[t]{2}{*}{$\begin{array}{l}\text { Birthweight } \\
\text { (grams) }\end{array}$} & \multicolumn{2}{|c|}{1996} & & & \multicolumn{2}{|c|}{$\begin{array}{l}\text { Year } \\
1998\end{array}$} & \multicolumn{2}{|c|}{1999} & \multicolumn{2}{|c|}{2000} \\
\hline & No. & $\%$ & No. & $\%$ & No. & $\%$ & No. & $\%$ & No. & $\%$ \\
\hline Less than 500 & 158 & 0.2 & 182 & 0.2 & 190 & 0.2 & 212 & 0.2 & 228 & 0.3 \\
\hline 500-999 & 362 & 0.4 & 414 & 0.5 & 398 & 0.5 & 391 & 0.4 & 425 & 0.5 \\
\hline $1000-1499$ & 454 & 0.5 & 467 & 0.5 & 481 & 0.6 & 509 & 0.6 & 546 & 0.6 \\
\hline 1500-1999 & 906 & 1.0 & 1033 & 1.2 & 1017 & 1.2 & 1076 & 1.2 & 1079 & 1.2 \\
\hline $2000-2499$ & 3158 & 3.7 & 3318 & 3.8 & 3147 & 3.6 & 3353 & 3.8 & 3383 & 3.8 \\
\hline 2500-2999 & 13098 & 15.2 & 13487 & 15.3 & 12810 & 14.8 & 12942 & 14.8 & 12819 & 14.6 \\
\hline 3000-3499 & 31378 & 36.3 & 31863 & 36.2 & 30974 & 35.9 & 30978 & 35.5 & 30647 & 34.9 \\
\hline 3500-3999 & 26793 & 31.0 & 26957 & 30.6 & 26818 & 31.1 & 27173 & 31.1 & 27483 & 31.3 \\
\hline $4000-4499$ & 8593 & 9.9 & 8816 & 10.0 & 8807 & 10.2 & 9002 & 10.3 & 9454 & 10.8 \\
\hline $4500+$ & 1477 & 1.7 & 1535 & 1.7 & 1597 & 1.9 & 1629 & 1.9 & 1811 & 2.1 \\
\hline Not stated & 52 & 0.1 & 61 & 0.1 & 66 & 0.1 & 24 & 0.0 & 47 & 0.1 \\
\hline TOTAL & 86429 & 100.0 & 88133 & 100.0 & 86305 & 100.0 & 87289 & 100.0 & 87922 & 100.0 \\
\hline
\end{tabular}

Source: NSW Midwives Data Collection (HOIST). Epidemiology and Surveillance Branch, NSW Department of Health. 


\subsection{APGAR SCORE}

In 2000, 2.3 per cent of babies were born with an Apgar score of less than seven at five minutes and 1.2 per cent were born with a score less than four (Table 16). These rates are similar to those of previous years.

\section{TABLE 16}

BIRTHS BY APGAR SCORE AT FIVE MINUTES, NSW 1996-2000\#

\begin{tabular}{|c|c|c|c|c|c|c|c|c|c|c|}
\hline \multirow[t]{2}{*}{ Apgar score } & \multicolumn{4}{|c|}{1996} & \multicolumn{2}{|c|}{$\begin{array}{c}\text { Year } \\
1998\end{array}$} & \multicolumn{2}{|c|}{1999} & \multicolumn{2}{|c|}{2000} \\
\hline & No. & $\%$ & No. & $\%$ & No. & $\%$ & No. & $\%$ & No. & $\%$ \\
\hline $0-4$ & 989 & 1.1 & 1065 & 1.2 & 1001 & 1.2 & 996 & 1.1 & 1043 & 1.2 \\
\hline $5-6$ & 1127 & 1.3 & 1116 & 1.3 & 990 & 1.1 & 1098 & 1.3 & 956 & 1.1 \\
\hline $7+$ & 84153 & 97.4 & 85788 & 97.3 & 84114 & 97.5 & 85028 & 97.4 & 85756 & 97.5 \\
\hline Not stated & 160 & 0.2 & 164 & 0.2 & 200 & 0.2 & 167 & 0.2 & 167 & 0.2 \\
\hline TOTAL & 86429 & 100.0 & 88133 & 100.0 & 86305 & 100.0 & 87289 & 100.0 & 87922 & 100.0 \\
\hline
\end{tabular}

Source: NSW Midwives Data Collection (HOIST). Epidemiology and Surveillance Branch, NSW Department of Health.

\# Includes stillbirths and live births.

\subsection{PERINATAL OUTCOMES}

In the period 1996-2000 the perinatal mortality rate varied from 8.8 to 9.4 per 1,000 (Table 17). In 2000, about twothirds of all perinatal deaths were stillbirths and one quarter were neonatal deaths.
In 2000, of the 852 perinatal deaths in NSW, 825 (96.8 per cent) were reported among planned hospital births, 9 (1.1 per cent) among planned birth centre births, none among planned home births, and 18 were among babies born before arrival at hospital.

\section{TABLE 17}

BIRTHS BY PERINATAL OUTCOME, NSW 1996-2000\#

\begin{tabular}{|c|c|c|c|c|c|c|c|c|c|c|c|}
\hline \multirow[t]{2}{*}{ Year } & \multirow{2}{*}{$\begin{array}{l}\text { Liveborn } \\
\text { surviving } \\
\text { No. }\end{array}$} & \multirow[b]{2}{*}{$\%$} & \multicolumn{2}{|c|}{ Stillborn } & \multicolumn{2}{|c|}{$\begin{array}{c}\text { Perinatal Outcome } \\
\text { Neonatal } \\
\text { death }\end{array}$} & \multicolumn{2}{|c|}{ Not stated } & \multicolumn{2}{|c|}{$\begin{array}{l}\text { Total } \\
\text { births }\end{array}$} & \multirow[t]{2}{*}{$\begin{array}{l}\text { Perinatal mortality } \\
\text { rate } / 1,000 \text { births }\end{array}$} \\
\hline & & & No. & $\%$ & No. & $\%$ & No. & $\%$ & No. & $\%$ & \\
\hline 1996 & 85627 & 99.1 & 545 & 0.6 & 227 & 0.3 & 30 & 0.0 & 86429 & 100.0 & 8.9 \\
\hline 1997 & 87200 & 98.9 & 587 & 0.7 & 262 & 0.3 & 84 & 0.1 & 88133 & 100.0 & 9.6 \\
\hline 1998 & 85376 & 98.9 & 595 & 0.7 & 216 & 0.3 & 118 & 0.1 & 86305 & 100.0 & 9.4 \\
\hline 1999 & 86468 & 99.1 & 533 & 0.6 & 271 & 0.3 & 17 & 0.0 & 87289 & 100.0 & 9.2 \\
\hline 2000 & 87066 & 99.0 & 595 & 0.7 & 257 & 0.3 & 4 & 0.0 & 87922 & 100.0 & 9.7 \\
\hline
\end{tabular}

Source: NSW Midwives Data Collection (HOIST). Epidemiology and Surveillance Branch, NSW Department of Health.

\# Perinatal deaths include deaths reported to the MDC only. As the MDC form is completed at discharge or transfer of the baby, deaths occurring after this time may not be reported to the MDC. 


\subsection{MATERNAL DEATHS}

In the period 1990-1998, 106 deaths were reported among pregnant women or women who gave birth less than six weeks previously. Of these, 36 (34.0 per cent) died of incidental causes not related to the pregnancy or its management; 51 (48.1 per cent) deaths were found to be directly due to pregnancy or its management; and 19 (17.9 per cent) deaths were found to result from pre-existing disease or disease which developed during pregnancy (not due to direct obstetric causes), but which may have been aggravated by the physiologic effects of pregnancy (Table 18).

\section{TABLE 18}

MATERNAL DEATHS BY YEAR, NSW 1990-2000\#

\begin{tabular}{|c|c|c|c|c|c|c|c|c|c|c|}
\hline \multirow[t]{2}{*}{ Year } & \multicolumn{2}{|c|}{ Direct } & \multicolumn{2}{|c|}{ Indirect } & \multicolumn{2}{|c|}{$\begin{array}{c}\text { Classification } \\
\text { Total } \\
\text { Direct \& Indirect }\end{array}$} & \multicolumn{2}{|c|}{ Incidental } & \multicolumn{2}{|c|}{ TOTAL } \\
\hline & No. & $\begin{array}{r}\text { Rate/ } \\
100,000\end{array}$ & No. & $\begin{array}{r}\text { Rate/ } \\
100,000\end{array}$ & No. & $\begin{array}{r}\text { Rate/ } \\
100,000\end{array}$ & No. & $\begin{array}{r}\text { Rate/ } \\
100,000\end{array}$ & No. & $\begin{array}{r}\text { Rate/ } \\
100,000\end{array}$ \\
\hline 1990 & 4 & 4.6 & 6 & 6.9 & 10 & 11.6 & 2 & 2.3 & 12 & 13.9 \\
\hline 1991 & 4 & 4.7 & 1 & 1.2 & 5 & 5.8 & 1 & 1.2 & 6 & 7.0 \\
\hline 1992 & 5 & 5.7 & 1 & 1.1 & 6 & 6.8 & 5 & 5.7 & 11 & 12.5 \\
\hline 1993 & 6 & 6.9 & 1 & 1.2 & 7 & 8.1 & 6 & 6.9 & 13 & 15.0 \\
\hline 1994 & 8 & 9.2 & 1 & 1.2 & 9 & 10.4 & 3 & 3.5 & 12 & 13.8 \\
\hline 1995 & 7 & 8.1 & 2 & 2.3 & 9 & 10.4 & 6 & 7.0 & 15 & 17.4 \\
\hline 1996 & 6 & 7.0 & 1 & 1.2 & 7 & 8.2 & 5 & 5.9 & 12 & 14.1 \\
\hline 1997 & 7 & 8.1 & 2 & 2.3 & 9 & 10.5 & 5 & 5.8 & 14 & 16.1 \\
\hline 1998 & 4 & 4.7 & 4 & 4.7 & 8 & 9.4 & 3 & 3.5 & 11 & 12.9 \\
\hline $1999^{\# \#}$ & & & & & & & & & 12 & 14.0 \\
\hline $2000^{\# \#}$ & & & & & & & & & 10 & 11.6 \\
\hline
\end{tabular}

Source: NSW Maternal and Perinatal Committee.

\# Includes all deaths of women who were pregnant at the time of death, or who died within 42 days of childbirth.

Direct deaths include those resulting from obstetric complications of the pregnant state, including its management.

Indirect deaths include those resulting from preexisting disease or disease which developed during pregnancy and was not due to direct obstetric causes but which may have been aggravated by the physiological effects of pregnancy.

Incidental deaths are those where the pregnancy is unlikely to have contributed significantly to the death. ${ }^{1}$

\#\#

Classification incomplete for 1999 and 2000.

\section{TABLE 19}

MATERNAL DEATHS BY CAUSE, NSW 1998

\begin{tabular}{|ll|}
\hline Classification & Cause \\
\hline Direct & Amniotic fluid embolism \\
Direct & Brainstem haemorrhage following pre-eclampsia \\
Direct & Ruptured ectopic pregnancy \\
Direct & Haemorrhage associated with disseminated intravascular coagulopathy following \\
& delivery of stillborn fetus \\
Indirect & Pulmonary hypertension and thrombotic thrombocytopenic purpura \\
Indirect & Cardiac and renal failure following sub-acute bacterial endocarditis \\
Indirect & Aortic valve disease \\
Indirect & Suicide following acute reactive depression \\
Incidental & Complications of Crohn disease \\
Incidental & Intracerebral haemorrhage \\
Incidental & Acute hepatitis B infection \\
TOTAL & \\
\hline
\end{tabular}

Source: NSW Maternal and Perinatal Committee.

\# Includes all deaths of women who were pregnant at the time of death, or who died within 42 days of childbirth.

Direct deaths include those resulting from obstetric complications of the pregnant state, including its management.

Indirect deaths include those resulting from preexisting disease or disease which developed during pregnancy and was not due to direct obstetric causes but which may have been aggravated by the physiological effects of pregnancy. ${ }^{1}$

Incidental deaths are those where the pregnancy is unlikely to have contributed significantly to the death.

\section{Reference}

1. National Health and Medical Research Council. Report on Maternal Deaths in Australia 1994-96. Canberra: NHMRC and AIHW National Perinatal Statistics Unit, 2001. 
PART 2: AREA HEALTH SERVICES

Information on the health of Aboriginal and Torres Strait Islander mothers, and mothers born in non-English speaking countries is shown in Parts 3 and 4 respectively.

\subsection{CONFINEMENTS}

Continuing the pattern of recent years, the largest numbers of confinements in 2000 were among mothers resident in the South Western Sydney (12,541, 14.5 per cent) and Western Sydney Health Areas (10,794, 12.5 per cent). These two health areas contributed over one quarter of the State's births. Eighty per cent of confinements were to mothers resident in the metropolitan Health Areas (including the Central Coast, Hunter and Illawarra Health Areas), and 19.1 per cent were to mothers resident in rural areas (Table 20).

\subsection{MATERNAL AGE}

The proportion of women giving birth at less than 20 years of age varied from 0.8 per cent in the Northern Sydney Health Area to 12.0 per cent in the Far West Health Area, while the proportion of mothers giving birth at 35 years of age or more ranged from 10.7 per cent in the Far West Health Area to 29.1 per cent in the Northern Sydney Health Area.

\subsection{MATERNAL COUNTRY OF BIRTH}

Seventy-nine per cent of women who gave birth in NSW in 2000 were born in English speaking countries, 11.6 per cent were born in Asian countries and 4.3 per cent were born in the Middle East or Africa (Table 21).

The highest proportions of mothers born in non-English speaking countries were in the Central Sydney and South Western Sydney Health Areas. In Central Sydney, the majority of mothers born in non-English speaking countries were born in North East Asia (12.2 per cent) and South East Asia (10.7 per cent). In South Western Sydney, the majority of mothers born in non-English speaking countries were born in South East Asia (17.0 per cent).

\subsection{MATERNAL ABORIGINALITY}

In 2000, 2.4 per cent of mothers were reported to be Aboriginal or Torres Strait Islander (Table 22). The proportion of Aboriginal or Torres Strait Islander mothers varied from 0.1 per cent in the Northern Sydney Area to 26.8 per cent in the Far West Area.

\subsection{PLACE OF BIRTH}

Ninety-six per cent of mothers chose to deliver in a hospital labour ward in 2000, compared to 3.7 per cent who planned a birth centre birth and 0.2 per cent who planned a homebirth (Table 23). Planned birth centre births were most common in the Hunter and Central Sydney Health Areas, and planned home births were most common in the Northern Rivers Health Area.

\subsection{LABOUR AND DELIVERY}

In 2000, the onset of labour was spontaneous in 64.9 per cent of confinements (Table 24). Labour was induced in 23.6 per cent of confinements and no labour (elective caesarean section) was reported in 11.5 per cent of confinements.

The rate of spontaneous onset of labour was highest among residents of the Southern Health Area (71.5 per cent). The highest rate of induction of labour was among residents of the Greater Murray Health Area (28.4 per cent).

About two-thirds of confinements were by normal vaginal birth, 10.7 per cent were instrumental and 21.3 per cent were by caesarean section (Table 25). The highest rate of normal vaginal birth was among residents of Far West Health Area (76.4 per cent), while the highest rate of instrumental delivery was among residents of South Eastern Sydney Health Area (15.0 per cent). The caesarean section rate varied from 16.0 per cent among mothers resident in the South Western Sydney Health Area to 26.5 per cent in the Northern Sydney and Mid Western Health Areas. 


\section{TABLE 20}

CONFINEMENTS BY MATERNAL AGE AND HEALTH AREA OF RESIDENCE, NSW 2000

\begin{tabular}{|c|c|c|c|c|c|c|c|c|c|c|c|c|c|c|c|c|c|c|}
\hline \multirow[t]{3}{*}{ Health Area } & \multicolumn{18}{|c|}{ Maternal age (years) } \\
\hline & \multicolumn{2}{|c|}{$12-19$} & \multicolumn{2}{|c|}{$20-24$} & \multicolumn{2}{|c|}{$25-29$} & \multicolumn{2}{|c|}{$30-34$} & \multicolumn{2}{|c|}{$35-39$} & \multicolumn{2}{|c|}{$40-44$} & \multicolumn{2}{|c|}{$45+$} & \multicolumn{2}{|c|}{ Not stated } & \multicolumn{2}{|c|}{ TOTAL } \\
\hline & No. & $\%$ & No. & $\%$ & No. & $\%$ & No. & $\%$ & No. & $\%$ & No. & $\%$ & No. & $\%$ & No. & $\%$ & No. & $\%$ \\
\hline Central Sydney & 157 & 2.3 & 740 & 10.9 & 1807 & 26.7 & 2331 & 34.4 & 1422 & 21.0 & 295 & 4.4 & 17 & 0.3 & 6 & 0.1 & 6775 & 100.0 \\
\hline Northern Sydney & 80 & 0.8 & 506 & 5.4 & 2176 & 23.1 & 3922 & 41.6 & 2308 & 24.5 & 425 & 4.5 & 14 & 0.1 & 1 & 0.0 & 9432 & 100.0 \\
\hline Western Sydney & 431 & 4.0 & 1767 & 16.4 & 3561 & 33.0 & 3316 & 30.7 & 1435 & 13.3 & 272 & 2.5 & 9 & 0.1 & 3 & 0.0 & 10794 & 100.0 \\
\hline Wentworth & 275 & 5.6 & 921 & 18.7 & 1750 & 35.6 & 1301 & 26.4 & 577 & 11.7 & 92 & 1.9 & 4 & 0.1 & 1 & 0.0 & 4921 & 100.0 \\
\hline South Western & & & & & & & & & & & & & & & & & & \\
\hline Sydney & 548 & 4.4 & 2363 & 18.8 & 4408 & 35.1 & 3450 & 27.5 & 1492 & 11.9 & 267 & 2.1 & 13 & 0.1 & 0 & 0.0 & 12541 & 100.0 \\
\hline Central Coast & 182 & 4.8 & 634 & 16.8 & 1243 & 33.0 & 1154 & 30.6 & 467 & 12.4 & 88 & 2.3 & 4 & 0.1 & 0 & 0.0 & 3772 & 100.0 \\
\hline Hunter & 409 & 5.9 & 1284 & 18.4 & 2440 & 35.0 & 1930 & 27.6 & 777 & 11.1 & 133 & 1.9 & 7 & 0.1 & 1 & 0.0 & 6981 & 100.0 \\
\hline Illawarra & 222 & 5.0 & 761 & 17.3 & 1525 & 34.6 & 1238 & 28.1 & 558 & 12.7 & 98 & 2.2 & 4 & 0.1 & 1 & 0.0 & 4407 & 100.0 \\
\hline South Eastern & & & & & & & & & & & & & & & & & & \\
\hline Sydney & 151 & 1.6 & 890 & 9.2 & 2755 & 28.4 & 3654 & 37.7 & 1906 & 19.7 & 331 & 3.4 & 10 & 0.1 & 0 & 0.0 & 9697 & 100.0 \\
\hline Northern Rivers & 210 & 7.6 & 527 & 19.1 & 879 & 31.8 & 703 & 25.4 & 374 & 13.5 & 71 & 2.6 & 2 & 0.1 & 0 & 0.0 & 2766 & 100.0 \\
\hline Mid North Coast & 246 & 8.8 & 594 & 21.2 & 886 & 31.6 & 699 & 24.9 & 309 & 11.0 & 65 & 2.3 & 3 & 0.1 & 0 & 0.0 & 2802 & 100.0 \\
\hline New England & 205 & 9.1 & 466 & 20.6 & 786 & 34.7 & 538 & 23.8 & 233 & 10.3 & 30 & 1.3 & 2 & 0.1 & 5 & 0.2 & 2265 & 100.0 \\
\hline Macquarie & 162 & 10.2 & 352 & 22.1 & 513 & 32.3 & 380 & 23.9 & 159 & 10.0 & 22 & 1.4 & $\overline{1}$ & 0.1 & 1 & 0.1 & 1590 & 100.0 \\
\hline Mid Western & 179 & 7.9 & 468 & 20.7 & 753 & 33.3 & 572 & 25.3 & 251 & 11.1 & 39 & 1.7 & 2 & 0.1 & 0 & 0.0 & 2264 & 100.0 \\
\hline Far West & 64 & 12.0 & 128 & 24.0 & 158 & 29.6 & 126 & 23.6 & 50 & 9.4 & 6 & 1.1 & 1 & 0.2 & 0 & 0.0 & 533 & 100.0 \\
\hline Greater Murray & 156 & 6.2 & 476 & 18.9 & 837 & 33.3 & 705 & 28.0 & 286 & 11.4 & 52 & 2.1 & 3 & 0.1 & 2 & 0.1 & 2517 & 100.0 \\
\hline Southern & 133 & 7.5 & 332 & 18.8 & 614 & 34.8 & 440 & 24.9 & 209 & 11.8 & 37 & 2.1 & 1 & 0.1 & 0 & 0.0 & 1766 & 100.0 \\
\hline Other-Not stated & 43 & 6.8 & 107 & 16.8 & 202 & 31.7 & 181 & 28.4 & 81 & 12.7 & 19 & 3.0 & 1 & 0.2 & 3 & 0.5 & 637 & 100.0 \\
\hline TOTAL & 3853 & 4.5 & 13316 & 15.4 & 27293 & 31.6 & 26640 & 30.8 & 12894 & 14.9 & 2342 & 2.7 & 98 & 0.1 & 24 & 0.0 & 86460 & 100.0 \\
\hline
\end{tabular}

Source: NSW Midwives Data Collection (HOIST). Epidemiology and Surveillance Branch, NSW Department of Health.

\section{TABLE 21}

CONFINEMENTS BY MATERNAL COUNTRY OF BIRTH AND HEALTH AREA OF RESIDENCE, NSW 2000"

Health Area

Country of birth group

English Central Melanesia, Southern Western Eastern Middle East South North Southern TOTAL

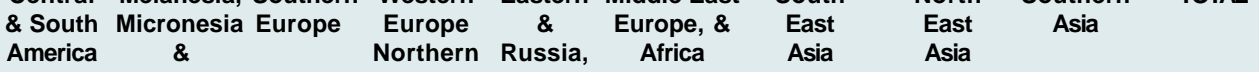

Polynesia

Europe Central

Asian \&

Baltic

No. $\%$ No. $\%$ No. $\%$ No. $\%$ No. $\%$ No. $\%$ No. $\%$ No. $\%$ No. $\%$ No. $\%$ No. $\%$

$\begin{array}{llllllllllllllllllllllll}\text { Central Sydney } & 3745 & 55.3 & 74 & 1.1 & 245 & 3.6 & 204 & 3.0 & 71 & 1.0 & 57 & 0.8 & 592 & 8.7 & 724 & 10.7 & 824 & 12.2 & 237 & 3.5 & 6773 & 100.0\end{array}$ $\begin{array}{lllllllllllllllllllllllll}\text { Northern Sydney } & 7367 & 78.1 & 89 & 0.9 & 111 & 1.2 & 102 & 1.1 & 189 & 2.0 & 71 & 0.8 & 227 & 2.4 & 381 & 4.0 & 685 & 7.3 & 209 & 2.2 & 9431 & 100.0\end{array}$ $\begin{array}{lllllllllllllllllllllll}\text { Western Sydney } & 6892 & 64.0 & 105 & 1.0 & 445 & 4.1 & 169 & 1.6 & 50 & 0.5 & 50 & 0.5 & 1039 & 9.6 & 815 & 7.6 & 683 & 6.3 & 527 & 4.9 & 10775 & 100.0\end{array}$ $\begin{array}{lllllllllllllllllllllllll} & 4471 & 90.9 & 20 & 0.4 & 67 & 1.4 & 54 & 1.1 & 36 & 0.7 & 14 & 0.3 & 65 & 1.3 & 110 & 2.2 & 28 & 0.6 & 55 & 1.1 & 4920 & 100.0\end{array}$ $\begin{array}{lllllllllllllllllllllll}\text { South Western } & 7489 & 59.7 & 237 & 1.9 & 449 & 3.6 & 320 & 2.6 & 56 & 0.4 & 75 & 0.6 & 1220 & 9.7 & 2129 & 17.0 & 370 & 3.0 & 194 & 1.5 & 12539 & 100.0\end{array}$

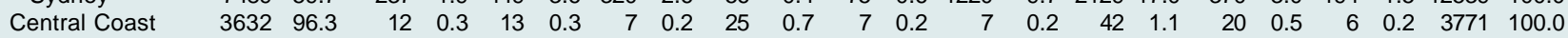
$\begin{array}{llllllllllllllllllllllll}\text { Hunter } & 6741 & 96.6 & 12 & 0.2 & 33 & 0.5 & 23 & 0.3 & 16 & 0.2 & 7 & 0.1 & 16 & 0.2 & 84 & 1.2 & 38 & 0.5 & 11 & 0.2 & 6981 & 100.0\end{array}$

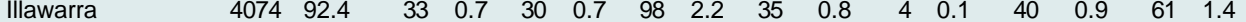

South Eastern Sydney Northern Rivers 708973.1

$\begin{array}{llllll}35 & 0.6 & 7 & 0.2 & 4407 & 100.0\end{array}$

$\begin{array}{llllllllllllllllllllll}2661 & 96.2 & 3 & 0.1 & 9 & 0.3 & 3 & 0.1 & 24 & 0.9 & 4 & 0.1 & 1 & 0.0 & 35 & 1.3 & 20 & 0.7 & 6 & 0.2 & 2766 & 100.0\end{array}$

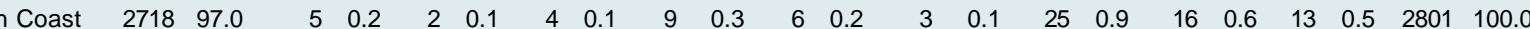
$\begin{array}{lllllllllllllllllllllll}\text { New England } & 2211 & 97.7 & 1 & 0.0 & 8 & 0.4 & 3 & 0.1 & 7 & 0.3 & 0 & 0.0 & 6 & 0.3 & 17 & 0.8 & 9 & 0.4 & 2 & 0.1 & 2264 & 100.0\end{array}$ $\begin{array}{llllllllllllllllllllllll}\text { Macquarie } & 1562 & 98.2 & 1 & 0.1 & 2 & 0.1 & 2 & 0.1 & 3 & 0.2 & 4 & 0.3 & 4 & 0.3 & 5 & 0.3 & 6 & 0.4 & 1 & 0.1 & 1590 & 100.0\end{array}$ $\begin{array}{llllllllllllllllllllllll}\text { Mid Western } & 2219 & 98.0 & 0 & 0.0 & 4 & 0.2 & 4 & 0.2 & 7 & 0.3 & 3 & 0.1 & 2 & 0.1 & 14 & 0.6 & 6 & 0.3 & 5 & 0.2 & 2264 & 100.0 \\ & & 518 & 97.2 & & 0.2 & 2 & 0.4 & 2 & 0.4 & 3 & 0.6 & 0 & 0.0 & 0 & 0.0 & 4 & 0.8 & 2 & 0.4 & 1 & 0.2 & 533 & 100.0\end{array}$

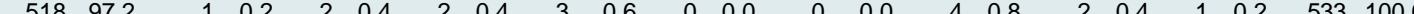
$\begin{array}{lllllllllllllllllllllll}\text { Greater Murray } & 2421 & 96.2 & 3 & 0.1 & 28 & 1.1 & 2 & 0.1 & 5 & 0.2 & 2 & 0.1 & 7 & 0.3 & 13 & 0.5 & 14 & 0.6 & 22 & 0.9 & 2517 & 100.0\end{array}$ $\begin{array}{llllllllllllllllllllllllll}\text { Southern } & 1697 & 96.1 & 2 & 0.1 & 10 & 0.6 & 7 & 0.4 & 13 & 0.7 & 1 & 0.1 & 9 & 0.5 & 15 & 0.8 & 8 & 0.5 & 4 & 0.2 & 1766 & 100.0\end{array}$ $\begin{array}{llllllllllllllllllllllll}\text { Other-Not stated } & 598 & 94.2 & 1 & 0.2 & 3 & 0.5 & 3 & 0.5 & 3 & 0.5 & 1 & 0.2 & 5 & 0.8 & 14 & 2.2 & 7 & 1.1 & 0 & 0.0 & 635 & 100.0\end{array}$ $\begin{array}{lllllllllllllllllllllll}\text { TOTAL } & 68105 & 78.8 & 708 & 0.8 & 1606 & 1.9 & 1217 & 1.4 & 671 & 0.8 & 428 & 0.5 & 3685 & 4.3 & 5085 & 5.9 & 3449 & 4.0 & 1476 & 1.7 & 86430 & 100.0\end{array}$

Source: NSW Midwives Data Collection (HOIST). Epidemiology and Surveillance Branch, NSW Department of Health.

\# Excludes 30 mothers for whom country of birth was not stated. Maternal countries of birth and country of birth groups are shown in Appendix 3. 


\section{TABLE 22}

CONFINEMENTS BY MATERNAL ABORIGINALITY AND HEALTH AREA OF RESIDENCE, NSW 2000

\begin{tabular}{|c|c|c|c|c|c|c|c|c|}
\hline \multirow[t]{3}{*}{ Health Area } & \multicolumn{8}{|c|}{ Aboriginality } \\
\hline & \multicolumn{2}{|c|}{$\begin{array}{l}\text { Aboriginal } \\
\text { Torres Strait } \\
\text { Islander }\end{array}$} & \multicolumn{2}{|c|}{$\begin{array}{l}\text { Non-Aboriginal } \\
\text { Torres Strait } \\
\text { Islander }\end{array}$} & \multicolumn{2}{|c|}{ Not stated } & \multicolumn{2}{|c|}{ TOTAL } \\
\hline & No. & $\%$ & No. & $\%$ & No. & $\%$ & No. & $\%$ \\
\hline Central Sydney & 69 & 1.0 & 6704 & 99.0 & 2 & 0.0 & 6775 & 100.0 \\
\hline Northern Sydney & 9 & 0.1 & 9421 & 99.9 & 2 & 0.0 & 9432 & 100.0 \\
\hline Western Sydney & 134 & 1.2 & 10625 & 98.4 & 35 & 0.3 & 10794 & 100.0 \\
\hline Wentworth & 64 & 1.3 & 4854 & 98.6 & 3 & 0.1 & 4921 & 100.0 \\
\hline South Western Sydney & 99 & 0.8 & 12441 & 99.2 & 1 & 0.0 & 12541 & 100.0 \\
\hline Central Coast & 72 & 1.9 & 3700 & 98.1 & 0 & 0.0 & 3772 & 100.0 \\
\hline Hunter & 156 & 2.2 & 6825 & 97.8 & 0 & 0.0 & 6981 & 100.0 \\
\hline Illawarra & 138 & 3.1 & 4269 & 96.9 & 0 & 0.0 & 4407 & 100.0 \\
\hline South Eastern Sydney & 35 & 0.4 & 9662 & 99.6 & 0 & 0.0 & 9697 & 100.0 \\
\hline Northern Rivers & 175 & 6.3 & 2591 & 93.7 & 0 & 0.0 & 2766 & 100.0 \\
\hline Mid North Coast & 218 & 7.8 & 2583 & 92.2 & 1 & 0.0 & 2802 & 100.0 \\
\hline New England & 255 & 11.3 & 2008 & 88.7 & 2 & 0.1 & 2265 & 100.0 \\
\hline Macquarie & 222 & 14.0 & 1367 & 86.0 & 1 & 0.1 & 1590 & 100.0 \\
\hline Mid Western & 124 & 5.5 & 2140 & 94.5 & 0 & 0.0 & 2264 & 100.0 \\
\hline Far West & 143 & 26.8 & 390 & 73.2 & 0 & 0.0 & 533 & 100.0 \\
\hline Greater Murray & 107 & 4.3 & 2410 & 95.7 & 0 & 0.0 & 2517 & 100.0 \\
\hline Southern & 69 & 3.9 & 1697 & 96.1 & 0 & 0.0 & 1766 & 100.0 \\
\hline Other-Not stated & 16 & 2.5 & 619 & 97.2 & 2 & 0.3 & 637 & 100.0 \\
\hline TOTAL & 2105 & 2.4 & 84306 & 97.5 & 49 & 0.1 & 86460 & 100.0 \\
\hline
\end{tabular}

Source: NSW Midwives Data Collection (HOIST). Epidemiology and Surveillance Branch, NSW Department of Health.

\section{TABLE 23}

CONFINEMENTS BY PLACE OF BIRTH AND HEALTH AREA OF RESIDENCE, NSW 2000

\begin{tabular}{|c|c|c|c|c|c|c|c|c|c|c|c|c|c|c|c|c|}
\hline \multirow[t]{2}{*}{ Health Area } & \multicolumn{2}{|c|}{ Hospital } & \multicolumn{2}{|c|}{$\begin{array}{l}\text { Birth } \\
\text { centre }\end{array}$} & \multicolumn{2}{|c|}{$\begin{array}{l}\text { Planned } \\
\text { birth centre- } \\
\text { hospital } \\
\text { admission }\end{array}$} & \multicolumn{2}{|c|}{$\begin{array}{l}\text { Planned } \\
\text { home } \\
\text { birth }\end{array}$} & \multicolumn{2}{|c|}{$\begin{array}{l}\text { Place of birth } \\
\text { Planned } \\
\text { home birth- } \\
\text { hospital } \\
\text { admission }\end{array}$} & \multicolumn{2}{|c|}{$\begin{array}{l}\text { Born } \\
\text { before } \\
\text { arrival }\end{array}$} & \multicolumn{2}{|c|}{$\begin{array}{c}\text { Not } \\
\text { stated }\end{array}$} & \multicolumn{2}{|c|}{ TOTAL } \\
\hline & No. & $\%$ & No. & $\%$ & No. & $\%$ & No. & $\%$ & No. & $\%$ & No. & $\%$ & No. & $\%$ & No. & $\%$ \\
\hline Central Sydney & 6147 & 90.7 & 406 & 6.0 & 174 & 2.6 & 10 & 0.1 & 1 & 0.0 & 37 & 0.5 & 0 & 0.0 & 6775 & 100.0 \\
\hline Northern Sydney & 9290 & 98.5 & 82 & 0.9 & 28 & 0.3 & 17 & 0.2 & 1 & 0.0 & 14 & 0.1 & 0 & 0.0 & 9432 & 100.0 \\
\hline Western Sydney & 10145 & 94.0 & 297 & 2.8 & 279 & 2.6 & 8 & 0.1 & 2 & 0.0 & 62 & 0.6 & 1 & 0.0 & 10794 & 100.0 \\
\hline Wentworth & 4828 & 98.1 & 28 & 0.6 & 42 & 0.9 & 6 & 0.1 & 2 & 0.0 & 14 & 0.3 & 1 & 0.0 & 4921 & 100.0 \\
\hline South Western Sydney & 12292 & 98.0 & 155 & 1.2 & 39 & 0.3 & 4 & 0.0 & 0 & 0.0 & 51 & 0.4 & 0 & 0.0 & 12541 & 100.0 \\
\hline Central Coast & 3734 & 99.0 & 13 & 0.3 & 1 & 0.0 & 1 & 0.0 & 1 & 0.0 & 22 & 0.6 & 0 & 0.0 & 3772 & 100.0 \\
\hline Hunter & 6185 & 88.6 & 668 & 9.6 & 91 & 1.3 & 5 & 0.1 & 1 & 0.0 & 31 & 0.4 & 0 & 0.0 & 6981 & 100.0 \\
\hline Illawarra & 4362 & 99.0 & 10 & 0.2 & 3 & 0.1 & 8 & 0.2 & 4 & 0.1 & 20 & 0.5 & 0 & 0.0 & 4407 & 100.0 \\
\hline South Eastern Sydney & 8907 & 91.9 & 492 & 5.1 & 252 & 2.6 & 16 & 0.2 & 4 & 0.0 & 26 & 0.3 & 0 & 0.0 & 9697 & 100.0 \\
\hline Northern Rivers & 2707 & 97.9 & 9 & 0.3 & 10 & 0.4 & 11 & 0.4 & 11 & 0.4 & 18 & 0.7 & 0 & 0.0 & 2766 & 100.0 \\
\hline Mid North Coast & 2764 & 98.6 & 16 & 0.6 & 10 & 0.4 & 0 & 0.0 & 1 & 0.0 & 11 & 0.4 & 0 & 0.0 & 2802 & 100.0 \\
\hline New England & 2248 & 99.2 & 5 & 0.2 & 0 & 0.0 & 0 & 0.0 & 2 & 0.1 & 10 & 0.4 & 0 & 0.0 & 2265 & 100.0 \\
\hline Macquarie & 1562 & 98.2 & 7 & 0.4 & 10 & 0.6 & 1 & 0.1 & 5 & 0.3 & 5 & 0.3 & 0 & 0.0 & 1590 & 100.0 \\
\hline Mid Western & 2232 & 98.6 & 4 & 0.2 & 11 & 0.5 & 2 & 0.1 & 2 & 0.1 & 13 & 0.6 & 0 & 0.0 & 2264 & 100.0 \\
\hline Far West & 526 & 98.7 & 0 & 0.0 & 0 & 0.0 & 1 & 0.2 & 0 & 0.0 & 6 & 1.1 & 0 & 0.0 & 533 & 100.0 \\
\hline Greater Murray & 2501 & 99.4 & 1 & 0.0 & 5 & 0.2 & 1 & 0.0 & 0 & 0.0 & 9 & 0.4 & 0 & 0.0 & 2517 & 100.0 \\
\hline Southern & 1732 & 98.1 & 9 & 0.5 & 3 & 0.2 & 9 & 0.5 & 1 & 0.1 & 12 & 0.7 & 0 & 0.0 & 1766 & 100.0 \\
\hline Other-Not stated & 620 & 97.3 & 3 & 0.5 & 1 & 0.2 & 8 & 1.3 & 0 & 0.0 & 5 & 0.8 & 0 & 0.0 & 637 & 100.0 \\
\hline TOTAL & 82782 & 95.7 & 2205 & 2.6 & 959 & 1.1 & 108 & 0.1 & 38 & 0.0 & 366 & 0.4 & 2 & 0.0 & 86460 & 100.0 \\
\hline
\end{tabular}

Source: NSW Midwives Data Collection (HOIST). Epidemiology and Surveillance Branch, NSW Department of Health. 


\section{TABLE 24}

CONFINEMENTS BY ONSET AND AUGMENTATION OF LABOUR AND HEALTH AREA OF RESIDENCE, NSW 2000

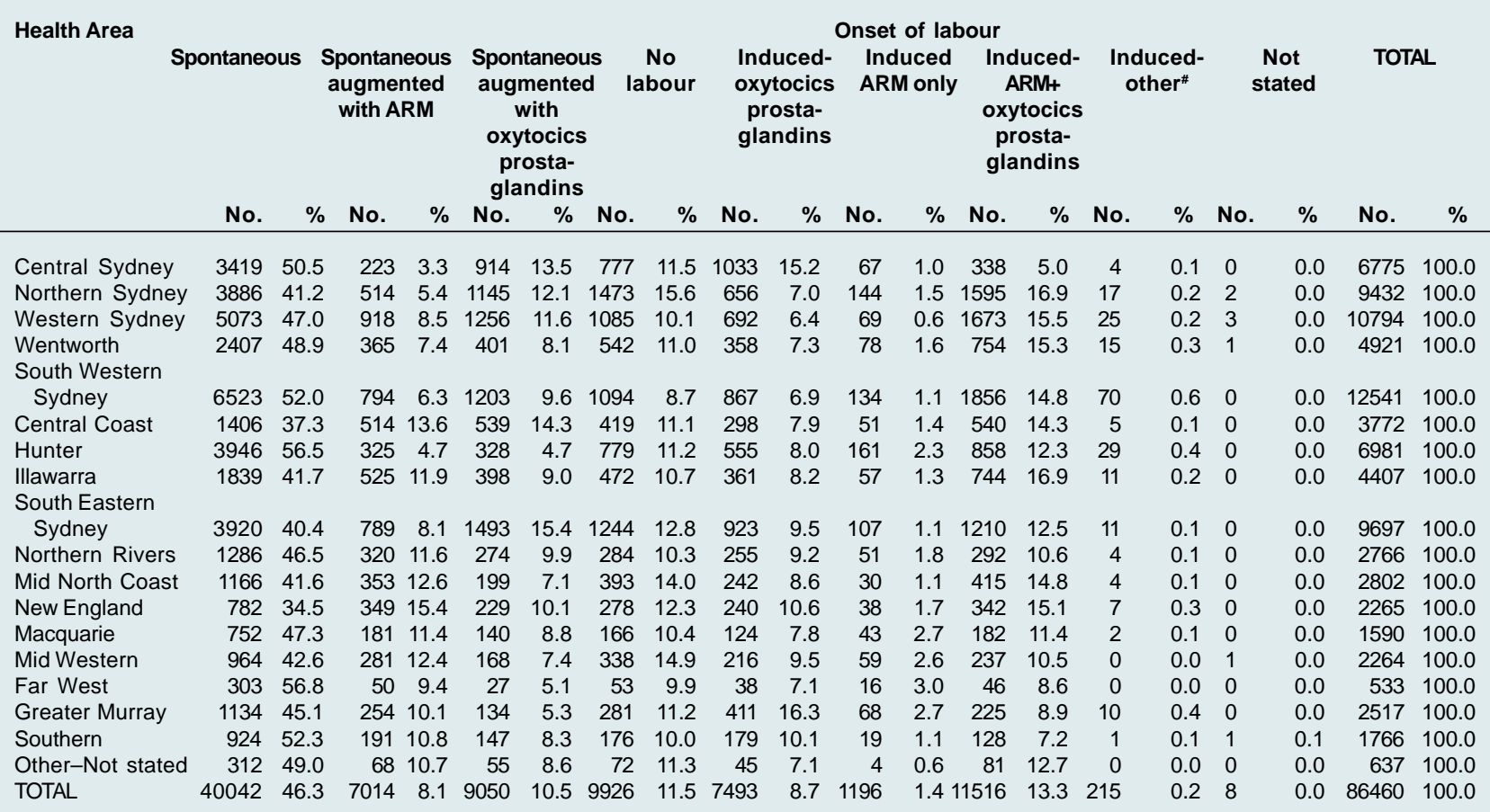

Source: NSW Midwives Data Collection (HOIST). Epidemiology and Surveillance Branch, NSW Department of Health.

\# This category includes other forms of induction such as Foley's catheter.

\section{TABLE 25}

CONFINEMENTS BY TYPE OF DELIVERY AND HEALTH AREA OF RESIDENCE, NSW 2000

\begin{tabular}{|c|c|c|c|c|c|c|c|c|c|c|c|c|c|c|c|c|}
\hline \multirow{3}{*}{ Health Area } & \multirow{2}{*}{\multicolumn{2}{|c|}{$\begin{array}{l}\text { Normal } \\
\text { vaginal }\end{array}$}} & \multirow{2}{*}{\multicolumn{2}{|c|}{ Forceps }} & \multirow{2}{*}{\multicolumn{2}{|c|}{$\begin{array}{l}\text { Vacuum } \\
\text { extraction }\end{array}$}} & \multicolumn{4}{|c|}{ Type of delivery } & \multirow{2}{*}{\multicolumn{2}{|c|}{$\begin{array}{l}\text { Emergency } \\
\text { caesarean } \\
\text { section }^{\#}\end{array}$}} & \multirow{2}{*}{\multicolumn{2}{|c|}{ Not stated }} & \multirow{2}{*}{\multicolumn{2}{|c|}{ TOTAL }} \\
\hline & & & & & & & & & $\begin{array}{l}\text { Elec } \\
\text { caes }\end{array}$ & tive & & & & & & \\
\hline & No. & $\%$ & No. & $\%$ & No. & $\%$ & No. & $\%$ & No. & $\%$ & No. & $\%$ & No. & $\%$ & No. & $\%$ \\
\hline Central Sydney & 4325 & 63.8 & 301 & 4.4 & 560 & 8.3 & 54 & 0.8 & 777 & 11.5 & 758 & 11.2 & 0 & 0.0 & 6775 & 100.0 \\
\hline Northern Sydney & 5525 & 58.6 & 540 & 5.7 & 824 & 8.7 & 39 & 0.4 & 1473 & 15.6 & 1029 & 10.9 & 2 & 0.0 & 9432 & 100.0 \\
\hline Western Sydney & 7512 & 69.6 & 684 & 6.3 & 415 & 3.8 & 97 & 0.9 & 1085 & 10.1 & 992 & 9.2 & 9 & 0.1 & 10794 & 100.0 \\
\hline Wentworth & 3460 & 70.3 & 194 & 3.9 & 208 & 4.2 & 30 & 0.6 & 542 & 11.0 & 485 & 9.9 & 2 & 0.0 & 4921 & 100.0 \\
\hline South Western Sydney & 9265 & 73.9 & 362 & 2.9 & 783 & 6.2 & 119 & 0.9 & 1094 & 8.7 & 918 & 7.3 & 0 & 0.0 & 12541 & 100.0 \\
\hline Central Coast & 2457 & 65.1 & 97 & 2.6 & 349 & 9.3 & 22 & 0.6 & 419 & 11.1 & 428 & 11.3 & 0 & 0.0 & 3772 & 100.0 \\
\hline Hunter & 4797 & 68.7 & 191 & 2.7 & 440 & 6.3 & 75 & 1.1 & 779 & 11.2 & 699 & 10.0 & 0 & 0.0 & 6981 & 100.0 \\
\hline Illawarra & 3066 & 69.6 & 135 & 3.1 & 310 & 7.0 & 31 & 0.7 & 472 & 10.7 & 393 & 8.9 & 0 & 0.0 & 4407 & 100.0 \\
\hline South Eastern Sydney & 5685 & 58.6 & 643 & 6.6 & 817 & 8.4 & 61 & 0.6 & 1244 & 12.8 & 1246 & 12.8 & 1 & 0.0 & 9697 & 100.0 \\
\hline Northern Rivers & 1978 & 71.5 & 103 & 3.7 & 84 & 3.0 & 22 & 0.8 & 284 & 10.3 & 295 & 10.7 & 0 & 0.0 & 2766 & 100.0 \\
\hline Mid North Coast & 1963 & 70.1 & 94 & 3.4 & 83 & 3.0 & 24 & 0.9 & 393 & 14.0 & 245 & 8.7 & 0 & 0.0 & 2802 & 100.0 \\
\hline New England & 1565 & 69.1 & 115 & 5.1 & 101 & 4.5 & 19 & 0.8 & 278 & 12.3 & 187 & 8.3 & 0 & 0.0 & 2265 & 100.0 \\
\hline Macquarie & 1151 & 72.4 & 92 & 5.8 & 59 & 3.7 & 11 & 0.7 & 166 & 10.4 & 111 & 7.0 & 0 & 0.0 & 1590 & 100.0 \\
\hline Mid Western & 1478 & 65.3 & 82 & 3.6 & 91 & 4.0 & 14 & 0.6 & 338 & 14.9 & 261 & 11.5 & 0 & 0.0 & 2264 & 100.0 \\
\hline Far West & 407 & 76.4 & 15 & 2.8 & 13 & 2.4 & 9 & 1.7 & 53 & 9.9 & 36 & 6.8 & 0 & 0.0 & 533 & 100.0 \\
\hline Greater Murray & 1697 & 67.4 & 156 & 6.2 & 126 & 5.0 & 23 & 0.9 & 281 & 11.2 & 234 & 9.3 & 0 & 0.0 & 2517 & 100.0 \\
\hline Southern & 1265 & 71.6 & 82 & 4.6 & 83 & 4.7 & 8 & 0.5 & 176 & 10.0 & 151 & 8.6 & 1 & 0.1 & 1766 & 100.0 \\
\hline Other-Not stated & 453 & 71.1 & 18 & 2.8 & 21 & 3.3 & 11 & 1.7 & 72 & 11.3 & 62 & 9.7 & 0 & 0.0 & 637 & 100.0 \\
\hline TOTAL & 58049 & 67.1 & 3904 & 4.5 & 5367 & 6.2 & 669 & 0.8 & 9926 & 11.5 & 8530 & 9.9 & 15 & 0.0 & 86460 & 100.0 \\
\hline
\end{tabular}

Source: NSW Midwives Data Collection (HOIST). Epidemiology and Surveillance Branch, NSW Department of Health.

\# Emergency caesarean section includes caesarean sections where the onset of labour was not stated. 


\subsection{BIRTHWEIGHT}

In 2000, 6.4 per cent of births were low birthweight (less than 2,500 grams). These comprised 0.7 per cent of birthweight less than 1,000 grams, 0.6 per cent in the 1,000 to 1,499 gram range, and 5.1 per cent in the 1,500 to 2,499 gram range (Table 26). The highest rate of low birthweight was in the Macquarie Health Area (8.1 per cent) and the lowest rate was in the Southern Health Area (4.4 per cent).

\subsection{GESTATIONAL AGE}

The majority of births (90.3 per cent) were at term and 2.4 per cent were post-term (42-plus weeks). The 7.3 per cent of preterm births comprised 0.7 per cent born at 20-27 weeks, 0.8 per cent at $28-31$ weeks and 5.8 per cent at $32-36$ weeks. The highest rate of preterm birth was in the Mid Western Health Area (9.0 per cent), while the lowest rate was 4.5 per cent in the Southern Health Area (Table 27).

\section{TABLE 26}

BIRTHS BY BIRTHWEIGHT AND HEALTH AREA OF RESIDENCE,NSW 2000

\begin{tabular}{|c|c|c|c|c|c|c|c|c|c|c|c|c|c|c|c|c|c|c|c|c|c|c|c|c|}
\hline \multirow[t]{3}{*}{ Health Area } & \multicolumn{24}{|c|}{ Birthweight (grams) } \\
\hline & \multicolumn{3}{|c|}{$\begin{array}{c}\text { Less than } \\
500\end{array}$} & $\begin{array}{c}500- \\
999\end{array}$ & \multicolumn{2}{|c|}{$\begin{array}{c}1000- \\
1499\end{array}$} & \multicolumn{2}{|c|}{$\begin{array}{c}1500- \\
1999\end{array}$} & \multicolumn{2}{|c|}{$\begin{array}{c}2000- \\
2499\end{array}$} & \multicolumn{2}{|c|}{$\begin{array}{c}2500- \\
2999\end{array}$} & \multirow{2}{*}{\multicolumn{2}{|c|}{$\begin{array}{c}3000- \\
3499 \\
\text { No. } \%\end{array}$}} & \multicolumn{2}{|c|}{$\begin{array}{c}3500- \\
3999\end{array}$} & \multicolumn{2}{|c|}{$\begin{array}{c}4000- \\
4499\end{array}$} & \multicolumn{2}{|c|}{$4500+$} & \multicolumn{2}{|c|}{$\begin{array}{c}\text { Not } \\
\text { stated }\end{array}$} & \multicolumn{2}{|c|}{ TOTAL } \\
\hline & No. & $\% 1$ & No. & $\%$ & No. & $\%$ & No. & $\%$ & No. & $\%$ & No. & $\%$ & & & No. & $\%$ & No. & $\%$ & No. & $\% N$ & No. & $\%$ & No. & $\%$ \\
\hline Central Sydney & 26 & 0.4 & 43 & 0.6 & 49 & 0.7 & 97 & 1.4 & 280 & 4.0 & 1106 & 16.0 & 2446 & 35.4 & 2104 & 30.4 & 649 & 9.4 & 112 & 1.6 & 4 & 0.1 & 6916 & 100.0 \\
\hline Northern Sydney & 26 & 0.3 & 34 & 0.4 & 48 & 0.5 & 84 & 0.9 & 308 & 3.2 & 1206 & 12.6 & 3341 & 34.8 & 3230 & 33.6 & 1140 & 11.9 & 181 & 1.9 & 2 & 0.0 & 9600 & 100.0 \\
\hline Western Sydney & 23 & 0.2 & 58 & 0.5 & 88 & 0.8 & 115 & 1.0 & 398 & 3.6 & 1660 & 15.2 & 3902 & 35.6 & 3329 & 30.4 & 1133 & 10.3 & 238 & 2.2 & 12 & 0.1 & 10956 & 100.0 \\
\hline Wentworth & 13 & 0.3 & 30 & 0.6 & 28 & 0.6 & 65 & 1.3 & 176 & 3.5 & 660 & 13.2 & 1684 & 33.6 & 1664 & 33.2 & 568 & 11.3 & 116 & 2.3 & 1 & 0.0 & 5005 & 100.0 \\
\hline South Western & & & & & & & & & & & & & & & & & & & & & & & & \\
\hline Sydney & 24 & 0.2 & 50 & 0.4 & 82 & 0.6 & 165 & 1.3 & 538 & 4.2 & 2038 & 16.0 & 4685 & 36.8 & 3726 & 29.2 & 1185 & 9.3 & 254 & 2.0 & 1 & 0.0 & 12748 & 100.0 \\
\hline Central Coast & 13 & 0.3 & 14 & 0.4 & 29 & 0.8 & 48 & 1.3 & 138 & 3.6 & 504 & 13.1 & 1207 & 31.5 & 1280 & 33.4 & 498 & 13.0 & 103 & 2.7 & 2 & 0.1 & 3836 & 100.0 \\
\hline Hunter & 31 & 0.4 & 47 & 0.7 & 62 & 0.9 & 98 & 1.4 & 267 & 3.8 & 978 & 13.8 & 2315 & 32.6 & 2227 & 31.4 & 886 & 12.5 & 182 & 2.6 & 2 & 0.0 & 7095 & 100.0 \\
\hline Illawarra & 12 & 0.3 & 27 & 0.6 & 21 & 0.5 & 57 & 1.3 & 173 & 3.9 & 612 & 13.6 & 1535 & 34.2 & 1467 & 32.7 & 496 & 11.0 & 88 & 2.0 & 1 & 0.0 & 4489 & 100.0 \\
\hline South Eastern & & & & & & & & & & & & & & & & & & & & & & & & \\
\hline Sydney & 21 & 0.2 & 44 & 0.4 & 48 & 0.5 & 123 & 1.2 & 368 & 3.7 & 1432 & 14.5 & 3605 & 36.5 & 3058 & 31.0 & 993 & 10.1 & 183 & 1.9 & 5 & 0.1 & 9880 & 100.0 \\
\hline Northern Rivers & 6 & 0.2 & 8 & 0.3 & 8 & 0.3 & 39 & 1.4 & 112 & 4.0 & 427 & 15.3 & 944 & 33.8 & 879 & 31.4 & 319 & 11.4 & 51 & 1.8 & 2 & 0.1 & 2795 & 100.0 \\
\hline Mid North Coast & 7 & 0.2 & 15 & 0.5 & 15 & 0.5 & 50 & 1.8 & 133 & 4.7 & 440 & 15.4 & 955 & 33.4 & 840 & 29.4 & 334 & 11.7 & 67 & 2.3 & 1 & 0.0 & 2857 & 100.0 \\
\hline New England & 7 & 0.3 & 16 & 0.7 & 19 & 0.8 & 29 & 1.3 & 101 & 4.4 & 369 & 16.1 & 828 & 36.0 & 655 & 28.5 & 236 & 10.3 & 36 & 1.6 & 2 & 0.1 & 2298 & 100.0 \\
\hline Macquarie & 2 & 0.1 & 6 & 0.4 & 12 & 0.7 & 20 & 1.2 & 92 & 5.7 & 219 & 13.5 & 548 & 33.7 & 517 & 31.8 & 173 & 10.7 & 33 & 2.0 & 2 & 0.1 & 1624 & 100.0 \\
\hline Mid Western & 4 & 0.2 & 14 & 0.6 & 17 & 0.7 & 35 & 1.5 & 103 & 4.5 & 355 & 15.4 & 766 & 33.1 & 757 & 32.7 & 220 & 9.5 & 39 & 1.7 & 2 & 0.1 & 2312 & 100.0 \\
\hline Far West & 0 & 0.0 & 5 & 0.9 & 1 & 0.2 & 5 & 0.9 & 28 & 5.2 & 74 & 13.8 & 200 & 37.4 & 153 & 28.6 & 53 & 9.9 & 14 & 2.6 & 2 & 0.4 & 535 & 100.0 \\
\hline Greater Murray & 5 & 0.2 & 10 & 0.4 & 10 & 0.4 & 24 & 0.9 & 84 & 3.3 & 372 & 14.6 & 864 & 33.9 & 832 & 32.6 & 292 & 11.4 & 57 & 2.2 & 1 & 0.0 & 2551 & 100.0 \\
\hline Southern & 4 & 0.2 & 2 & 0.1 & 3 & 0.2 & 14 & 0.8 & 56 & 3.1 & 274 & 15.4 & 587 & 32.9 & 584 & 32.8 & 209 & 11.7 & 46 & 2.6 & 3 & 0.2 & 1782 & 100.0 \\
\hline Other-Not stated & 4 & 0.6 & 2 & 0.3 & 6 & 0.9 & 11 & 1.7 & 28 & 4.4 & 93 & 14.5 & 235 & 36.5 & 181 & 28.1 & 70 & 10.9 & 11 & 1.7 & 2 & 0.3 & 643 & 100.0 \\
\hline TOTAL & 228 & 0.3 & 425 & 0.5 & 546 & 0.6 & 1079 & 1.2 & 3383 & 3.8 & 12819 & 14.6 & 30647 & 34.9 & 27483 & 31.3 & 9454 & 10.8 & 811 & 2.1 & 47 & 0.1 & 87922 & 100.0 \\
\hline
\end{tabular}

Source: NSW Midwives Data Collection (HOIST). Epidemiology and Surveillance Branch, NSW Department of Health.

\section{TABLE 27}

BIRTHS BY GESTATIONAL AGE AND HEALTH AREA OF RESIDENCE, NSW 2000

\begin{tabular}{|c|c|c|c|c|c|c|c|c|c|c|c|c|c|c|}
\hline \multirow[t]{3}{*}{ Health Area } & \multicolumn{14}{|c|}{ Gestational age (weeks) } \\
\hline & \multicolumn{2}{|c|}{$20-27$} & \multicolumn{2}{|c|}{$28-31$} & \multicolumn{2}{|c|}{$32-36$} & \multicolumn{2}{|c|}{$37-41$} & \multicolumn{2}{|c|}{$42+$} & \multicolumn{2}{|c|}{ Not stated } & \multicolumn{2}{|c|}{ TOTAL } \\
\hline & No. & $\%$ & No. & $\%$ & No. & $\%$ & No. & $\%$ & No. & $\%$ & No. & $\%$ & No. & $\%$ \\
\hline Central Sydney & 68 & 1.0 & 58 & 0.8 & 424 & 6.1 & 6261 & 90.5 & 105 & 1.5 & 0 & 0.0 & 6916 & 100.0 \\
\hline Northern Sydney & 59 & 0.6 & 47 & 0.5 & 499 & 5.2 & 8680 & 90.4 & 315 & 3.3 & 0 & 0.0 & 9600 & 100.0 \\
\hline Western Sydney & 74 & 0.7 & 94 & 0.9 & 567 & 5.2 & 9958 & 90.9 & 258 & 2.4 & 5 & 0.0 & 10956 & 100.0 \\
\hline Wentworth & 42 & 0.8 & 33 & 0.7 & 272 & 5.4 & 4520 & 90.3 & 138 & 2.8 & 0 & 0.0 & 5005 & 100.0 \\
\hline South Western & & & & & & & & & & & & & & \\
\hline Sydney & 68 & 0.5 & 90 & 0.7 & 748 & 5.9 & 11449 & 89.8 & 393 & 3.1 & 0 & 0.0 & 12748 & 100.0 \\
\hline Central Coast & 25 & 0.7 & 33 & 0.9 & 273 & 7.1 & 3472 & 90.5 & 33 & 0.9 & 0 & 0.0 & 3836 & 100.0 \\
\hline Hunter & 68 & 1.0 & 78 & 1.1 & 452 & 6.4 & 6121 & 86.3 & 376 & 5.3 & 0 & 0.0 & 7095 & 100.0 \\
\hline Illawarra & 39 & 0.9 & 31 & 0.7 & 251 & 5.6 & 4053 & 90.3 & 115 & 2.6 & 0 & 0.0 & 4489 & 100.0 \\
\hline South Eastern & & & & & & & & & & & & & & \\
\hline Sydney & 62 & 0.6 & 73 & 0.7 & 626 & 6.3 & 9022 & 91.3 & 97 & 1.0 & 0 & 0.0 & 9880 & 100.0 \\
\hline Northern Rivers & 15 & 0.5 & 9 & 0.3 & 165 & 5.9 & 2527 & 90.4 & 79 & 2.8 & 0 & 0.0 & 2795 & 100.0 \\
\hline Mid North Coast & 21 & 0.7 & 21 & 0.7 & 190 & 6.7 & 2567 & 89.8 & 58 & 2.0 & 0 & 0.0 & 2857 & 100.0 \\
\hline New England & 25 & 1.1 & 24 & 1.0 & 127 & 5.5 & 2103 & 91.5 & 19 & 0.8 & 0 & 0.0 & 2298 & 100.0 \\
\hline Macquarie & 10 & 0.6 & 11 & 0.7 & 90 & 5.5 & 1490 & 91.7 & 23 & 1.4 & 0 & 0.0 & 1624 & 100.0 \\
\hline Mid Western & 13 & 0.6 & 31 & 1.3 & 163 & 7.1 & 2072 & 89.6 & 33 & 1.4 & 0 & 0.0 & 2312 & 100.0 \\
\hline Far West & 5 & 0.9 & 2 & 0.4 & 25 & 4.7 & 498 & 93.1 & 5 & 0.9 & 0 & 0.0 & 535 & 100.0 \\
\hline Greater Murray & 14 & 0.5 & 16 & 0.6 & 123 & 4.8 & 2362 & 92.6 & 35 & 1.4 & 1 & 0.0 & 2551 & 100.0 \\
\hline Southern & 6 & 0.3 & 5 & 0.3 & 69 & 3.9 & 1651 & 92.6 & 51 & 2.9 & 0 & 0.0 & 1782 & 100.0 \\
\hline Other-Not stated & 9 & 1.4 & 7 & 1.1 & 50 & 7.8 & 562 & 87.4 & 15 & 2.3 & 0 & 0.0 & 643 & 100.0 \\
\hline TOTAL & 623 & 0.7 & 663 & 0.8 & 5114 & 5.8 & 79368 & 90.3 & 2148 & 2.4 & 6 & 0.0 & 87922 & 100.0 \\
\hline
\end{tabular}

Source: NSW Midwives Data Collection (HOIST). Epidemiology and Surveillance Branch, NSW Department of Health. 


\subsection{PERINATAL OUTCOMES}

The perinatal mortality rate in 2000 was 9.7 per 1,000 births. This rate includes all births and deaths of babies of at least 400 grams birthweight or at least 20 weeks gestation (Table 28). The rate varied from 5.6 per 1,000 in the Southern Health Area to 13.2 per 1,000 in the Hunter Health Area.

\section{TABLE 28}

PERINATAL OUTCOMES BY HEALTH AREA OF RESIDENCE, NSW $2000^{\#}$

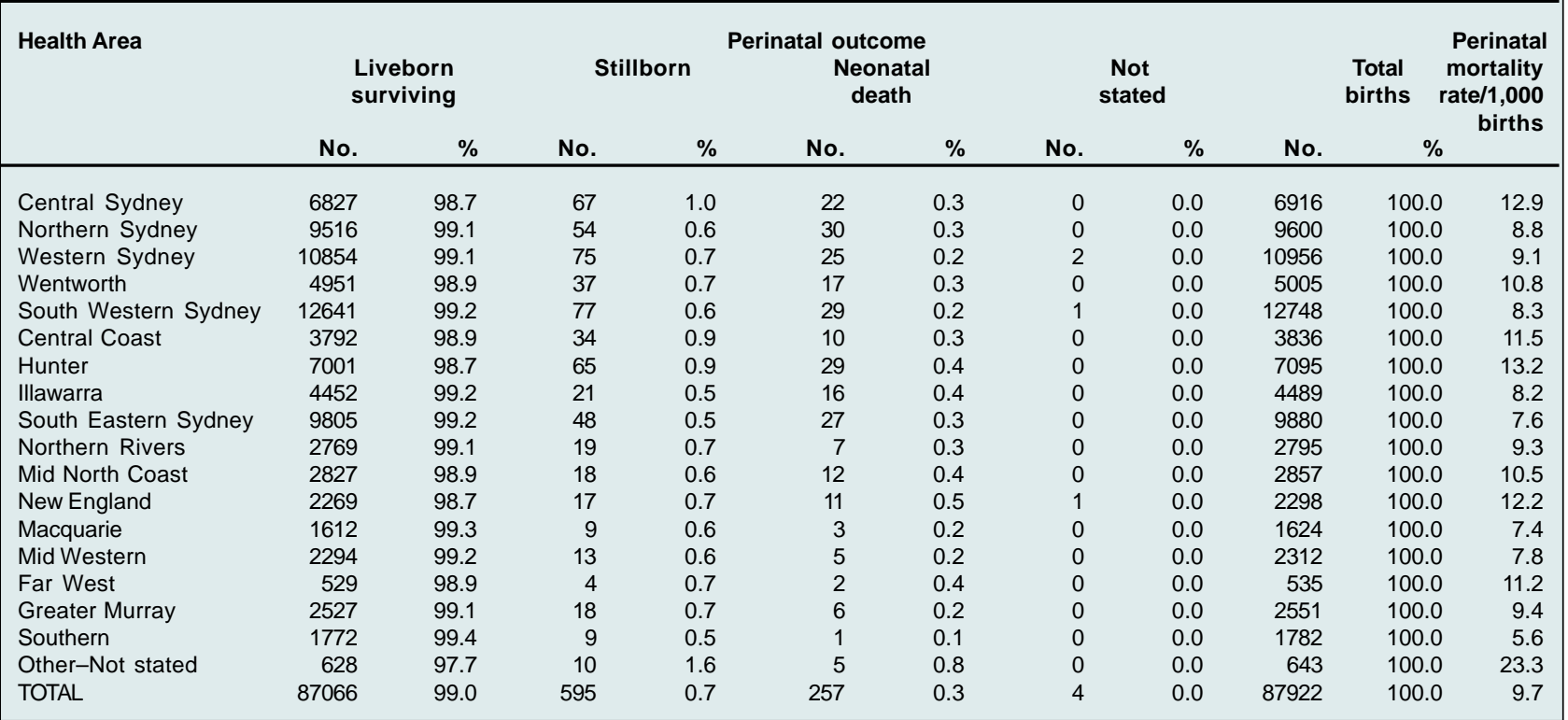

Source: NSW Midwives Data Collection (HOIST). Epidemiology and Surveillance Branch, NSW Department of Health.

\# Perinatal deaths include deaths reported to the MDC only. As the MDC form is completed at discharge or transfer of the baby, deaths occurring after this time may not be reported to the MDC. 


\section{PART 3: ABORIGINAL AND TORRES STRAIT ISLANDER MOTHERS AND BABIES}

\subsection{REPORTING OF ABORIGINALITY}

Maternal Aboriginality is under-reported on the MDC. One method of assessing the extent of under-reporting and monitoring improvements over time is to compare the reporting on maternal Aboriginality as reported to the MDC with information on birth registrations reported to the NSW Registry of Birth Deaths and Marriages. Using capture-recapture methods, an estimate of the total number of Aboriginal mothers can be obtained and compared with the number of Aboriginal mothers reported to the MDC. The method used here is described in Chapter 1 (page 16).
Using capture-recapture methods, the percentage of the estimated total number of Aboriginal mothers reported to the MDC rose from 58.7 to 65.7 per cent in the period 1994 to 1999 (Table 29). Reporting was better in rural hospitals than urban hospitals: it is estimated that in 199944.9 per cent of births to Aboriginal mothers in urban hospitals were correctly reported as Aboriginal compared to 88.5 per cent in rural hospitals.

\section{TABLE 29}

BIRTHS TO ABORIGINAL AND TORRES STRAIT ISLANDER MOTHERS BY SOURCE OF BIRTH REPORT, YEAR OF BIRTH AND URBAN-RURAL HEALTH AREA OF HOSPITAL, NSW 1994-1999

\begin{tabular}{|c|c|c|c|c|c|c|}
\hline $\begin{array}{l}\text { Urban-Rural } \\
\text { locality of } \\
\text { hospital- } \\
\text { Year\# }\end{array}$ & $\begin{array}{c}\text { MDC } \\
\text { births } \\
\text { No. }\end{array}$ & $\begin{array}{c}\text { RBDM } \\
\text { births } \\
\text { No. }\end{array}$ & $\begin{array}{l}\text { Births } \\
\text { reported } \\
\text { to both } \\
\text { MDC-RBDM } \\
\text { No. }\end{array}$ & $\begin{array}{c}\text { Estimated } \\
\text { Aboriginal } \\
\text { births } \\
\text { No. }\end{array}$ & $\begin{array}{c}\text { Estimated } \\
\text { Aboriginal } \\
\text { births } \\
\text { reported to MDC } \\
\%\end{array}$ & $\begin{array}{l}95 \% \text { confidence } \\
\text { interval of estimated } \\
\text { births reported }\end{array}$ \\
\hline \multicolumn{7}{|l|}{ Urban } \\
\hline 1994 & 553 & 665 & 268 & 1371 & 40.3 & $37.7-42.9$ \\
\hline 1995 & 642 & 742 & 345 & 1380 & 46.5 & $43.9-49.2$ \\
\hline 1996 & 593 & 794 & 338 & 1392 & 42.6 & $40.0-45.2$ \\
\hline 1997 & 658 & 1066 & 441 & 1590 & 41.4 & $39.0-43.8$ \\
\hline 1998 & 785 & 1053 & 495 & 1669 & 47.0 & $44.6-49.4$ \\
\hline 1999 & 706 & 995 & 447 & 1571 & 44.9 & $42.5-47.4$ \\
\hline \multicolumn{7}{|l|}{ Rural } \\
\hline 1994 & 990 & 747 & 561 & 1318 & 75.1 & $72.8-77.4$ \\
\hline 1995 & 1117 & 887 & 689 & 1438 & 77.7 & $75.5-79.8$ \\
\hline 1996 & 1131 & 941 & 679 & 1567 & 72.2 & $70.0-74.4$ \\
\hline 1997 & 1196 & 1011 & 789 & 1532 & 78.0 & $76.0-80.1$ \\
\hline 1998 & 1280 & 901 & 771 & 1496 & 85.6 & $83.8-87.4$ \\
\hline 1999 & 1372 & 906 & 802 & 1550 & 88.5 & $86.9-90.1$ \\
\hline \multicolumn{7}{|l|}{ NSW } \\
\hline 1994 & 1543 & 1412 & 829 & 2628 & 58.7 & $56.8-60.6$ \\
\hline 1995 & 1759 & 1629 & 1034 & 2771 & 63.5 & $61.7-65.3$ \\
\hline 1996 & 1724 & 1735 & 1017 & 2941 & 58.6 & $56.8-60.4$ \\
\hline 1997 & 1854 & 2077 & 1230 & 3130 & 59.2 & $57.5-60.9$ \\
\hline 1998 & 2065 & 1954 & 1266 & 3187 & 64.8 & $63.1-66.5$ \\
\hline 1999 & 2078 & 1901 & 1249 & 3162 & 65.7 & $64.1-67.4$ \\
\hline
\end{tabular}

Source: Linked NSW Midwives Data Collection and Registry of Births, Deaths and Marriages birth registration data.

\# 'Urban' and 'Rural' refer to urban or rural Health Area of Hospital as reported to the MDC. Urban hospitals include those in the following health areas: Central Sydney, Northern Sydney, Western Sydney, Wentworth, South Western Sydney, Central Coast, Hunter and IIlawarra. NSW totals exclude homebirths, and births for which the hospital of birth is not stated. 


\section{FIGURE3}

BIRTHS TO ABORIGINAL AND TORRES STRAIT ISLANDER MOTHERS BY YEAR OF BIRTH AND URBAN-RURAL HEALTH AREA OF HOSPITAL NSW 1994-1999"

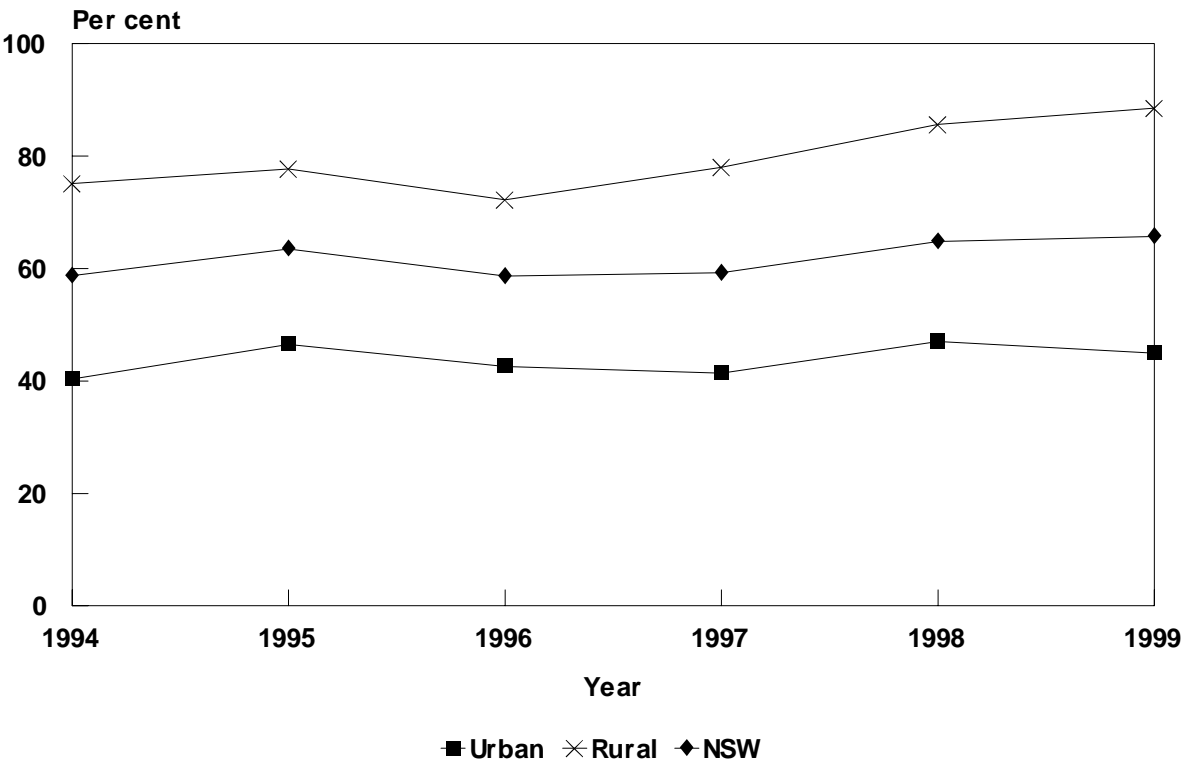

Source: Linked NSW Midwives Data Collection and Registry of Births, Deaths and Marriages birth registration data.

Urban' and 'Rural' refer to urban or rural Health Area of Hospital as reported to the MDC. Urban hospitals include those in the following health areas: Central Sydney, Northern Sydney, Western Sydney, Wentworth, South Western Sydney, Central Coast, Hunter and IIlawarra. NSW totals exclude homebirths and births for which area health service of hospital is not stated.

\subsection{TRENDS IN BIRTHS}

Between 1996 and 2000, the reported number of babies born to Aboriginal and Torres Strait Islander mothers increased from 1,724 to 2,122 (Table 30), an increase from 2.0 to 2.4 per cent of all babies born in NSW. Multiple pregnancies (twins, triplets etc.) were reported for about one per cent of mothers.

\section{TABLE 30}

ABORIGINAL AND TORRES STRAIT ISLANDER MOTHERS AND BABIES BY PLURALITY, NSW 1996-2000

\begin{tabular}{|c|c|c|c|c|c|c|c|c|c|c|}
\hline \multirow[t]{2}{*}{ Plurality } & \multicolumn{2}{|c|}{1996} & & 97 & \multicolumn{2}{|c|}{$\begin{array}{l}\text { Year } \\
1998\end{array}$} & \multicolumn{2}{|c|}{1999} & \multicolumn{2}{|c|}{2000} \\
\hline & No. & $\%$ & No. & $\%$ & No. & $\%$ & No. & $\%$ & No. & $\%$ \\
\hline \multicolumn{11}{|c|}{ Confinements } \\
\hline Singleton & 1700 & 99.3 & 1828 & 99.2 & 2017 & 98.7 & 2040 & 99.1 & 2089 & 99.2 \\
\hline Multiple & 12 & 0.7 & 14 & 0.8 & 26 & 1.3 & 19 & 0.9 & 16 & 0.8 \\
\hline TOTAL & 1712 & 100.0 & 1842 & 100.0 & 2043 & 100.0 & 2059 & 100.0 & 2105 & 100.0 \\
\hline \multicolumn{11}{|l|}{ Births } \\
\hline Singleton & 1700 & 98.6 & 1828 & 98.6 & 2017 & 97.5 & 2040 & 98.2 & 2089 & 98.4 \\
\hline Multiple & 24 & 1.4 & 26 & 1.4 & 51 & 2.5 & 38 & 1.8 & 33 & 1.6 \\
\hline TOTAL & 1724 & 100.0 & 1854 & 100.0 & 2068 & 100.0 & 2078 & 100.0 & 2122 & 100.0 \\
\hline
\end{tabular}

Source: NSW Midwives Data Collection (HOIST). Epidemiology and Surveillance Branch, NSW Department of Health. 


\subsection{PREVIOUS PREGNANCIES}

In 2000, about 30 per cent of Aboriginal and Torres Strait Islander mothers gave birth for the first time, and 61 per cent gave birth to their second to fourth baby (Table 31). Eight per cent of mothers had given birth to five or more babies. This pattern has not changed substantially since 1996.

\section{TABLE 31}

NUMBER OF PREVIOUS PREGNANCIES AMONG ABORIGINAL AND TORRES STRAIT ISLANDER MOTHERS, NSW 1996-2000

\begin{tabular}{|c|c|c|c|c|c|c|c|c|c|c|}
\hline \multirow[t]{2}{*}{$\begin{array}{l}\text { No. previous } \\
\text { pregnancies (>20 weeks) }\end{array}$} & \multicolumn{2}{|c|}{1996} & \multicolumn{2}{|c|}{1997} & \multicolumn{2}{|c|}{$\begin{array}{l}\text { Year } \\
1998\end{array}$} & \multicolumn{2}{|c|}{1999} & \multicolumn{2}{|c|}{2000} \\
\hline & No. & $\%$ & No. & $\%$ & No. & $\%$ & No. & $\%$ & No. & $\%$ \\
\hline 0 & 510 & 29.8 & 554 & 30.1 & 599 & 29.3 & 613 & 29.8 & 645 & 30.6 \\
\hline $1-4$ & 1065 & 62.2 & 1147 & 62.3 & 1280 & 62.7 & 1301 & 63.2 & 1285 & 61.0 \\
\hline $5+$ & 134 & 7.8 & 139 & 7.5 & 161 & 7.9 & 144 & 7.0 & 174 & 8.3 \\
\hline Not stated & 3 & 0.2 & 2 & 0.1 & 3 & 0.1 & 1 & 0.0 & 1 & 0.0 \\
\hline TOTAL & 1712 & 100.0 & 1842 & 100.0 & 2043 & 100.0 & 2059 & 100.0 & 2105 & 100.0 \\
\hline
\end{tabular}

Source: NSW Midwives Data Collection (HOIST). Epidemiology and Surveillance Branch, NSW Department of Health.

\subsection{MATERNAL AGE}

The reported number of babies born to Aboriginal and Torres Strait Islander mothers has increased at all ages. About one in five Aboriginal and Torres Strait Islander mothers were teenagers in 2000. Following statewide trends, the number of mothers giving birth at 35 years of age or more has increased over the last five years. The proportion of mothers aged 35-plus years increased from 4.8 in 1996 to 7.4 per cent in 2000 (Table 32).

\section{TABLE 32}

AGE OF ABORIGINAL AND TORRES STRAIT ISLANDER MOTHERS, NSW 1996-2000

\begin{tabular}{|c|c|c|c|c|c|c|c|c|c|c|}
\hline \multirow[t]{2}{*}{$\begin{array}{l}\text { Maternal age } \\
\text { (years) }\end{array}$} & \multicolumn{2}{|c|}{1996} & \multicolumn{2}{|c|}{1997} & \multicolumn{2}{|c|}{$\begin{array}{l}\text { Year } \\
1998\end{array}$} & \multicolumn{2}{|c|}{1999} & \multicolumn{2}{|c|}{2000} \\
\hline & No. & $\%$ & No. & $\%$ & No. & $\%$ & No. & $\%$ & No. & $\%$ \\
\hline $12-19$ & 374 & 21.8 & 398 & 21.6 & 389 & 19.0 & 443 & 21.5 & 459 & 21.8 \\
\hline $20-34$ & 1255 & 73.3 & 1352 & 73.4 & 1536 & 75.2 & 1492 & 72.5 & 1491 & 70.8 \\
\hline $35+$ & 83 & 4.8 & 92 & 5.0 & 113 & 5.5 & 124 & 6.0 & 155 & 7.4 \\
\hline Not stated & 0.0 & 0.0 & 0.0 & 0.0 & 5 & 0.2 & 0.0 & 0.0 & 0.0 & 0.0 \\
\hline TOTAL & 1712 & 100.0 & 1842 & 100.0 & 2043 & 100.0 & 2059 & 100.0 & 2105 & 100.0 \\
\hline
\end{tabular}

Source: NSW Midwives Data Collection (HOIST). Epidemiology and Surveillance Branch, NSW Department of Health. 


\subsection{HEALTH AREA OF RESIDENCE}

The reported number of Aboriginal and Torres Strait Islander mothers who gave birth in 2000 ranged from nine (0.4 per cent) in the Northern Sydney Area to 255 (12.1 per cent) in the New England Area (Table 33). Over one-third (36.9 per cent) of mothers were resident in urban health areas and about two-thirds were resident in rural health areas (62.4 per cent).

In 2000, over one quarter of Aboriginal and Torres Strait Islander mothers in the Mid North Coast, Far West and Southern Areas were teenagers (Table 34).

\begin{tabular}{|c|c|c|c|c|c|c|c|c|c|c|}
\hline \multirow[t]{3}{*}{ Health Area } & \multirow{2}{*}{\multicolumn{2}{|c|}{1996}} & \multirow{2}{*}{\multicolumn{2}{|c|}{1997}} & \multirow{2}{*}{\multicolumn{2}{|c|}{$\begin{array}{l}\text { Year } \\
1998\end{array}$}} & \multirow{2}{*}{\multicolumn{2}{|c|}{1999}} & \multirow{2}{*}{\multicolumn{2}{|c|}{2000}} \\
\hline & & & & & & & & & & \\
\hline & No. & $\%$ & No. & $\%$ & No. & $\%$ & No. & $\%$ & No. & $\%$ \\
\hline Central Sydney & 66 & 3.9 & 70 & 3.8 & 71 & 3.5 & 61 & 3.0 & 69 & 3.3 \\
\hline Northern Sydney & 9 & 0.5 & 7 & 0.4 & 10 & 0.5 & 9 & 0.4 & 9 & 0.4 \\
\hline Western Sydney & 108 & 6.3 & 105 & 5.7 & 172 & 8.4 & 139 & 6.8 & 134 & 6.4 \\
\hline Wentworth & 43 & 2.5 & 47 & 2.6 & 77 & 3.8 & 74 & 3.6 & 64 & 3.0 \\
\hline South Western Sydney & 93 & 5.4 & 89 & 4.8 & 108 & 5.3 & 91 & 4.4 & 99 & 4.7 \\
\hline Central Coast & 27 & 1.6 & 37 & 2.0 & 42 & 2.1 & 50 & 2.4 & 72 & 3.4 \\
\hline Hunter & 100 & 5.8 & 107 & 5.8 & 103 & 5.0 & 98 & 4.8 & 156 & 7.4 \\
\hline Illawarra & 101 & 5.9 & 125 & 6.8 & 119 & 5.8 & 104 & 5.1 & 138 & 6.6 \\
\hline South Eastern Sydney & 30 & 1.8 & 36 & 2.0 & 47 & 2.3 & 45 & 2.2 & 35 & 1.7 \\
\hline Northern Rivers & 132 & 7.7 & 146 & 7.9 & 161 & 7.9 & 162 & 7.9 & 175 & 8.3 \\
\hline Mid North Coast & 157 & 9.2 & 181 & 9.8 & 167 & 8.2 & 230 & 11.2 & 218 & 10.4 \\
\hline New England & 238 & 13.9 & 255 & 13.8 & 267 & 13.1 & 273 & 13.3 & 255 & 12.1 \\
\hline Macquarie & 171 & 10.0 & 202 & 11.0 & 212 & 10.4 & 230 & 11.2 & 222 & 10.5 \\
\hline Mid Western & 111 & 6.5 & 99 & 5.4 & 113 & 5.5 & 123 & 6.0 & 124 & 5.9 \\
\hline Far West & 157 & 9.2 & 172 & 9.3 & 169 & 8.3 & 162 & 7.9 & 143 & 6.8 \\
\hline Greater Murray & 120 & 7.0 & 100 & 5.4 & 120 & 5.9 & 116 & 5.6 & 107 & 5.1 \\
\hline Southern & 39 & 2.3 & 51 & 2.8 & 64 & 3.1 & 68 & 3.3 & 69 & 3.3 \\
\hline Other-Not stated & 10 & 0.6 & 13 & 0.7 & 21 & 1.0 & 24 & 1.2 & 16 & 0.8 \\
\hline TOTAL & 1712 & 100.0 & 1842 & 100.0 & 2043 & 100.0 & 2059 & 100.0 & 2105 & 100.0 \\
\hline
\end{tabular}

Source: NSW Midwives Data Collection (HOIST). Epidemiology and Surveillance Branch, NSW Department of Health.

\section{TABLE 34}

HEALTH AREA OF RESIDENCE OF ABORIGINAL AND TORRES STRAIT ISLANDER MOTHERS BY AGE, NSW 2000

\begin{tabular}{|c|c|c|c|c|c|c|}
\hline \multirow[t]{3}{*}{ Health Area } & \multicolumn{6}{|c|}{ Maternal age (years) } \\
\hline & \multicolumn{2}{|c|}{ Less than 20} & \multicolumn{2}{|c|}{$20+$} & \multicolumn{2}{|c|}{ TOTAL } \\
\hline & No. & $\%$ & No. & $\%$ & No. & $\%$ \\
\hline Central Sydney & 16 & 23.2 & 53 & 76.8 & 69 & 100.0 \\
\hline Northern Sydney\# & - & - & 8 & 88.9 & 9 & 100.0 \\
\hline Western Sydney & 38 & 28.4 & 96 & 71.6 & 134 & 100.0 \\
\hline Wentworth & 8 & 12.5 & 56 & 87.5 & 64 & 100.0 \\
\hline South Western Sydney & 22 & 22.2 & 77 & 77.8 & 99 & 100.0 \\
\hline Central Coast & 13 & 18.1 & 59 & 81.9 & 72 & 100.0 \\
\hline Hunter & 44 & 28.2 & 112 & 71.8 & 156 & 100.0 \\
\hline Illawarra & 24 & 17.4 & 114 & 82.6 & 138 & 100.0 \\
\hline South Eastern Sydney\# & - & - & 31 & 88.6 & 35 & 100.0 \\
\hline Northern Rivers & 28 & 16.0 & 147 & 84.0 & 175 & 100.0 \\
\hline Mid North Coast & 48 & 22.0 & 170 & 78.0 & 218 & 100.0 \\
\hline New England & 58 & 22.7 & 197 & 77.3 & 255 & 100.0 \\
\hline Macquarie & 55 & 24.8 & 167 & 75.2 & 222 & 100.0 \\
\hline Mid Western & 26 & 21.0 & 98 & 79.0 & 124 & 100.0 \\
\hline Far West & 36 & 25.2 & 107 & 74.8 & 143 & 100.0 \\
\hline Greater Murray & 21 & 19.6 & 86 & 80.4 & 107 & 100.0 \\
\hline Southern & 14 & 20.3 & 55 & 79.7 & 69 & 100.0 \\
\hline Other-Not stated & 3 & 18.8 & 13 & 81.3 & 16 & 100.0 \\
\hline TOTAL & 459 & 21.8 & 1646 & 78.2 & 2105 & 100.0 \\
\hline
\end{tabular}

Source: NSW Midwives Data Collection (HOIST). Epidemiology and Surveillance Branch, NSW Department of Health.

\# Information not shown for Health Areas where the number of mothers is less than five in a group. 


\subsection{BOOKING STATUS}

Between 1996 and 2000, there was no substantial change in the proportion of mothers who were booked into the hospital of birth-88.3 per cent in 1996 and 87.5 per cent in 2000 .

This compares with 98.1 per cent of non-Aboriginal or Torres Strait Islander mothers who were booked into the hospital of birth in 2000 .

\subsection{DURATION OF PREGNANCY AT FIRST ANTENATAL VISIT}

Between 1996 and 2000, there was a slight increase in the proportion of mothers who commenced antenatal care at less than 20 weeks gestation-from 61.0 per cent in 1996 to 67.6 per cent in 2000 . This compares with 87.0 per cent of non-Aboriginal and Torres Strait Islander mothers who commenced antenatal care at less than 20 weeks gestation in 2000 .

In 2000, the proportion of Aboriginal and Torres Strait Islander mothers who commenced antenatal care at less than 20 weeks gestation varied from 46.3 per cent in the Western Sydney Area to 86.1 per cent in the Central Coast Area (Table 35).

\section{TABLE 35}

DURATION OF PREGNANCY AT FIRST ANTENATAL VISIT AMONG ABORIGINAL AND TORRES STRAIT ISLANDER MOTHERS BY HEALTH AREA OF RESIDENCE, NSW 2000

\begin{tabular}{|c|c|c|c|c|c|c|c|c|}
\hline \multirow[t]{3}{*}{ Health Area } & \multicolumn{8}{|c|}{ Duration of pregnancy at first antenatal visit (weeks) } \\
\hline & \multicolumn{2}{|c|}{$0-19$} & \multirow{2}{*}{\multicolumn{2}{|c|}{$20+$}} & \multicolumn{2}{|c|}{ Not stated } & \multicolumn{2}{|c|}{ TOTAL } \\
\hline & No. & $\%$ & & $\%$ & No. & $\%$ & No. & $\%$ \\
\hline Central Sydney & 42 & 60.9 & 25 & 36.2 & 2 & 2.9 & 69 & 100.0 \\
\hline Northern Sydney\# & - & - & - & - & - & - & 9 & 100.0 \\
\hline Western Sydney & 62 & 46.3 & 59 & 44.0 & 13 & 9.7 & 134 & 100.0 \\
\hline Wentworth & 42 & 65.6 & 20 & 31.3 & 2 & 3.1 & 64 & 100.0 \\
\hline South Western Sydney & 51 & 51.5 & 44 & 44.4 & 4 & 4.0 & 99 & 100.0 \\
\hline Central Coast & 62 & 86.1 & 10 & 13.9 & 0 & 0.0 & 72 & 100.0 \\
\hline Hunter & 113 & 72.4 & 38 & 24.4 & 5 & 3.2 & 156 & 100.0 \\
\hline Illawarra & 97 & 70.3 & 37 & 26.8 & 4 & 2.9 & 138 & 100.0 \\
\hline South Eastern Sydney & 21 & 60.0 & 11 & 31.4 & 3 & 8.6 & 35 & 100.0 \\
\hline Northern Rivers & 113 & 64.6 & 52 & 29.7 & 10 & 5.7 & 175 & 100.0 \\
\hline Mid North Coast & 165 & 75.7 & 32 & 14.7 & 21 & 9.6 & 218 & 100.0 \\
\hline New England & 198 & 77.6 & 46 & 18.0 & 11 & 4.3 & 255 & 100.0 \\
\hline Macquarie & 144 & 64.9 & 57 & 25.7 & 21 & 9.5 & 222 & 100.0 \\
\hline Mid Western & 83 & 66.9 & 27 & 21.8 & 14 & 11.3 & 124 & 100.0 \\
\hline Far West & 82 & 57.3 & 43 & 30.1 & 18 & 12.6 & 143 & 100.0 \\
\hline Greater Murray & 81 & 75.7 & 24 & 22.4 & 2 & 1.9 & 107 & 100.0 \\
\hline Southern & 48 & 69.6 & 15 & 21.7 & $\overline{6}$ & 8.7 & 69 & 100.0 \\
\hline Other-Not stated & 12 & 75.0 & 3 & 18.8 & 1 & 6.3 & 16 & 100.0 \\
\hline TOTAL & 1422 & 67.6 & 546 & 25.9 & 137 & 6.5 & 2105 & 100.0 \\
\hline
\end{tabular}

Source: NSW Midwives Data Collection (HOIST). Epidemiology and Surveillance Branch, NSW Department of Health.

\# Information not shown for Health Areas where the number of mothers is less than five in a group. 


\subsection{SMOKING IN PREGNANCY}

In 2000, 55.9 per cent of Aboriginal and Torres Strait Islander mothers reported smoking at some time during pregnancy, compared to 61.4 per cent in 1996. This compares with 17.4 per cent of all mothers who reported smoking at some time during pregnancy in 2000 (see Section 1.8, page 21).
Smoking in the second half of pregnancy poses the greatest risk to the health of both mother and baby. In 2000, 55.2 per cent of Aboriginal and Torres Strait Islander mothers reported smoking in the second half of pregnancy. This percentage varied from 31.9 per cent in the Central Sydney Area to 71.0 per cent in the Southern Area (Figure 4).

\section{FIGURE4}

SMOKING IN THE SECOND HALF OF PREGNANCY AMONG ABORIGINAL AND TORRES STRAIT ISLANDER MOTHERS BY AMOUNT SMOKED AND HEALTH AREA OF RESIDENCE, NSW 2000

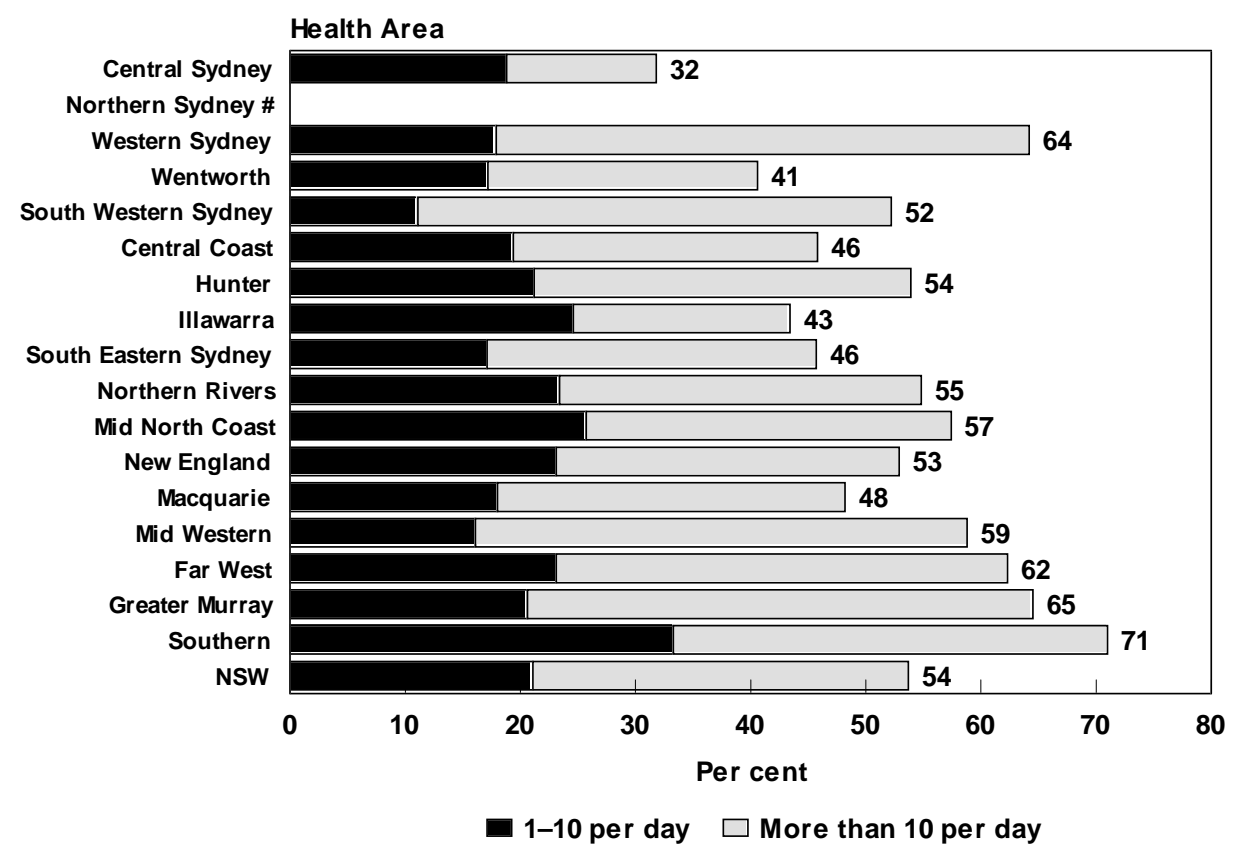

Source: NSW Midwives Data Collection (HOIST). Epidemiology and Surveillance Branch, NSW Department of Health.

\# Information not shown for Health Areas where the number of mothers is less than five in a group. 


\subsection{MEDICAL CONDITIONS AND OBSTETRIC COMPLICATIONS}

In 2000, there were similar rates of diabetes and essential hypertension reported among Aboriginal and Torres Strait
Islander mothers and non-Aboriginal or Torres Strait Islander mothers, and slightly lower rates of gestational diabetes and pregnancy-induced hypertension (Table 36).

\section{TABLE 36}

MATERNAL MEDICAL CONDITIONS AND OBSTETRIC COMPLICATIONS BY ABORIGINALITY, NSW 2000

\begin{tabular}{|c|c|c|c|c|c|c|}
\hline \multirow[t]{2}{*}{ Condition } & \multicolumn{2}{|c|}{$\begin{array}{l}\text { Aboriginality } \\
\text { Aboriginal and } \\
\text { Torres Strait } \\
\text { Islander }\end{array}$} & \multicolumn{2}{|c|}{$\begin{array}{l}\text { Non-Aboriginal or } \\
\text { Torres Strait } \\
\text { Islander }\end{array}$} & \multicolumn{2}{|c|}{ TOTAL } \\
\hline & No. & $\%$ & No. & $\%$ & No. & $\%$ \\
\hline Diabetes mellitus & 17 & 0.8 & 374 & 0.4 & 392 & 0.5 \\
\hline Gestational diabetes & 60 & 2.9 & 3326 & 3.9 & 3386 & 3.9 \\
\hline Essential hypertension & 23 & 1.1 & 834 & 1.0 & 858 & 1.0 \\
\hline Pregnancy- induced hypertension & 114 & 5.4 & 5967 & 7.1 & 6082 & 7.0 \\
\hline TOTAL CONFINEMENTS & 2105 & 100.0 & 84306 & 100.0 & 86460 & 100.0 \\
\hline
\end{tabular}

Source: NSW Midwives Data Collection (HOIST). Epidemiology and Surveillance Branch, NSW Department of Health.

\# Total confinements include 49 confinements where maternal Aboriginality was not stated.

\subsection{LABOUR AND DELIVERY}

The rate of induction of labour among Aboriginal and Torres Strait Islander mothers increased from 16.6 to 17.7 per cent between 1996 and 2000, while the rate of spontaneous onset of labour decreased from 76.8 to 72.5 per cent (Table 37).

These trends follow statewide trends (Section 1.10, page 24). However, the rate of induction of labour among
Aboriginal and Torres Strait Islander mothers continued to be lower than the NSW rate, which was 23.6 per cent in 2000.

Since 1996, the rate of normal vaginal birth fell slightly from 77.5 to 74.7 per cent. The caesarean section rate rose from 16.0 to 18.2 per cent (Table 38). Over the five year period, the percentage of deliveries by vacuum extraction almost doubled from 1.8 to 3.2 per cent.

\section{TABLE 37}

LABOUR ONSET FOR ABORIGINAL AND TORRES STRAIT ISLANDER MOTHERS, NSW 1996-2000

\begin{tabular}{|c|c|c|c|c|c|c|c|c|c|c|}
\hline \multirow[t]{2}{*}{ Labour onset } & \multicolumn{4}{|c|}{1996} & \multicolumn{2}{|c|}{$\begin{array}{c}\text { Year } \\
1998\end{array}$} & \multicolumn{2}{|c|}{1999} & \multicolumn{2}{|c|}{2000} \\
\hline & No. & $\%$ & No. & $\%$ & No. & $\%$ & No. & $\%$ & No. & $\%$ \\
\hline Spontaneous & 1315 & 76.8 & 1377 & 74.8 & 1467 & 71.8 & 1512 & 73.4 & 1527 & 72.5 \\
\hline No labour ${ }^{\#}$ & 112 & 6.5 & 153 & 8.3 & 176 & 8.6 & 172 & 8.4 & 206 & 9.8 \\
\hline Induced & 284 & 16.6 & 311 & 16.9 & 400 & 19.6 & 375 & 18.2 & 372 & 17.7 \\
\hline Not stated & 1 & 0.1 & 1 & 0.1 & 0 & 0.0 & 0 & 0.0 & 0 & 0.0 \\
\hline TOTAL & 1712 & 100.0 & 1842 & 100.0 & 2043 & 100.0 & 2059 & 100.0 & 2105 & 100.0 \\
\hline
\end{tabular}

Source: NSW Midwives Data Collection (HOIST). Epidemiology and Surveillance Branch, NSW Department of Health.

\# No labour indicates elective caesarean section.

\section{TABLE 38}

TYPE OF DELIVERY AMONG ABORIGINAL AND TORRES STRAIT ISLANDER MOTHERS, NSW 1996-2000

\begin{tabular}{|c|c|c|c|c|c|c|c|c|c|c|}
\hline \multirow[t]{2}{*}{ Type of delivery } & \multicolumn{2}{|c|}{1996} & \multicolumn{2}{|c|}{1997} & \multicolumn{2}{|c|}{$\begin{array}{c}\text { Year } \\
1998\end{array}$} & \multicolumn{2}{|c|}{1999} & \multicolumn{2}{|c|}{2000} \\
\hline & No. & $\%$ & No. & $\%$ & No. & $\%$ & No. & $\%$ & No. & $\%$ \\
\hline Normal vaginal & 1327 & 77.5 & 1423 & 77.3 & 1563 & 76.5 & 1586 & 77.0 & 1573 & 74.7 \\
\hline Forceps & 51 & 3.0 & 47 & 2.6 & 56 & 2.7 & 64 & 3.1 & 51 & 2.4 \\
\hline Vacuum extraction & 31 & 1.8 & 45 & 2.4 & 43 & 2.1 & 54 & 2.6 & 67 & 3.2 \\
\hline Vaginal breech & 28 & 1.6 & 15 & 0.8 & 27 & 1.3 & 25 & 1.2 & 31 & 1.5 \\
\hline Elective caesarean section & 112 & 6.5 & 153 & 8.3 & 176 & 8.6 & 172 & 8.4 & 206 & 9.8 \\
\hline Emergency caesarean section ${ }^{\#}$ & 162 & 9.5 & 159 & 8.6 & 177 & 8.7 & 158 & 7.7 & 177 & 8.4 \\
\hline Not stated & 1 & 0.1 & 0 & 0.0 & 1 & 0.0 & 0 & 0.0 & 0 & 0.0 \\
\hline TOTAL & 1712 & 100.0 & 1842 & 100.0 & 2043 & 100.0 & 2059 & 100.0 & 2105 & 100.0 \\
\hline
\end{tabular}

Source: NSW Midwives Data Collection (HOIST). Epidemiology and Surveillance Branch, NSW Department of Health.

\# Emergency caesarean section includes caesarean sections where the onset of labour was not stated. 


\subsection{BIRTHWEIGHT}

Since 1996, the rate of low birthweight (less than 2,500 grams) in Aboriginal and Torres Strait Islander babies has been over 10 per cent and was 11.9 per cent in 2000 (Table 39). This is almost twice the rate for NSW overall, which was 6.4 per cent in 2000. In 2000, the highest rate of low birthweight was 19.4 per cent in Mid Western Health Areas (Table 40).

\section{TABLE 39}

WEIGHT OF ABORIGINAL AND TORRES STRAIT ISLANDER BABIES, NSW 1996-2000

\begin{tabular}{|c|c|c|c|c|c|c|c|c|c|c|}
\hline \multirow[t]{2}{*}{$\begin{array}{l}\text { Birthweight } \\
\text { (grams) }\end{array}$} & \multicolumn{4}{|c|}{1996} & \multicolumn{2}{|c|}{$\begin{array}{c}\text { Year } \\
1998\end{array}$} & \multicolumn{2}{|c|}{1999} & \multicolumn{2}{|c|}{2000} \\
\hline & No. & $\%$ & No. & $\%$ & No. & $\%$ & No. & $\%$ & No. & $\%$ \\
\hline Less than 1,000 & 25 & 1.5 & 30 & 1.6 & 24 & 1.2 & 20 & 1.0 & 33 & 1.6 \\
\hline $1,000-1,499$ & 15 & 0.9 & 18 & 1.0 & 19 & 0.9 & 24 & 1.2 & 20 & 0.9 \\
\hline $1,500-2,499$ & 143 & 8.3 & 175 & 9.4 & 174 & 8.4 & 217 & 10.4 & 199 & 9.4 \\
\hline $2,500+$ & 1538 & 89.2 & 1631 & 88.0 & 1850 & 89.5 & 1816 & 87.4 & 1866 & 87.9 \\
\hline Not stated & 3 & 0.2 & 0 & 0.0 & 1 & 0.0 & 1 & 0.0 & 4 & 0.2 \\
\hline TOTAL & 1724 & 100.0 & 1854 & 100.0 & 2068 & 100.0 & 2078 & 100.0 & 2122 & 100.0 \\
\hline
\end{tabular}

Source: NSW Midwives Data Collection (HOIST). Epidemiology and Surveillance Branch, NSW Health Department.

\section{TABLE 40}

WEIGHT OF ABORIGINAL AND TORRES STRAIT ISLANDER BABIES BY HEALTH AREA OF RESIDENCE, NSW 2000

\begin{tabular}{|c|c|c|c|c|c|c|c|c|}
\hline \multirow[t]{3}{*}{ Health Area } & \multicolumn{8}{|c|}{ Birthweight (grams) } \\
\hline & \multicolumn{2}{|c|}{ Less than 2,500} & \multicolumn{2}{|c|}{$2,500+$} & \multicolumn{2}{|c|}{ Not stated } & & TOTAL \\
\hline & No. & $\%$ & No. & $\%$ & No. & $\%$ & No. & $\%$ \\
\hline Central Sydney & 7 & 10.1 & 62 & 89.9 & 0 & 0.0 & 69 & 100.0 \\
\hline Northern Sydney ${ }^{\#}$ & - & - & - & - & - & - & 9 & 100.0 \\
\hline Western Sydney & 23 & 16.8 & 114 & 83.2 & 0 & 0.0 & 137 & 100.0 \\
\hline Wentworth & 5 & 7.7 & 60 & 92.3 & 0 & 0.0 & 65 & 100.0 \\
\hline South Western Sydney & 10 & 10.1 & 89 & 89.9 & 0 & 0.0 & 99 & 100.0 \\
\hline Central Coast & 10 & 13.7 & 63 & 86.3 & 0 & 0.0 & 73 & 100.0 \\
\hline Hunter & 20 & 12.7 & 137 & 87.3 & 0 & 0.0 & 157 & 100.0 \\
\hline Illawarra & 12 & 8.7 & 126 & 91.3 & 0 & 0.0 & 138 & 100.0 \\
\hline South Eastern Sydney\# & - & - & - & - & - & 0.0 & 36 & 100.0 \\
\hline Northern Rivers & 20 & 11.4 & 155 & 88.1 & 1 & 0.6 & 176 & 100.0 \\
\hline Mid North Coast & 19 & 8.7 & 200 & 91.3 & 0 & 0.0 & 219 & 100.0 \\
\hline New England & 26 & 10.1 & 231 & 89.9 & 0 & 0.0 & 257 & 100.0 \\
\hline Macquarie & 30 & 13.5 & 191 & 85.7 & 2 & 0.9 & 223 & 100.0 \\
\hline Mid Western & 24 & 19.4 & 100 & 80.6 & 0 & 0.0 & 124 & 100.0 \\
\hline Far West & 17 & 11.8 & 126 & 87.5 & 1 & 0.7 & 144 & 100.0 \\
\hline Greater Murray & 11 & 9.9 & 100 & 90.1 & 0 & 0.0 & 111 & 100.0 \\
\hline Southern & 8 & 11.6 & 61 & 88.4 & 0 & 0.0 & 69 & 100.0 \\
\hline Other-Not stated & 5 & 31.3 & 11 & 68.8 & 0 & 0.0 & 16 & 100.0 \\
\hline TOTAL & 252 & 11.9 & 1866 & 87.9 & 4 & 0.2 & 2122 & 100.0 \\
\hline
\end{tabular}

Source: NSW Midwives Data Collection (HOIST). Epidemiology and Surveillance Branch, NSW Department of Health.

\# Information not shown for Health Areas where the number of mothers is less than five in a group. 


\subsection{GESTATIONAL AGE}

Since 1996, the rate of prematurity (less than 37 weeks gestation) in Aboriginal and Torres Strait Islander babies has been over 10 per cent. The rate was 11.6 per cent in 2000 (Table 41) — about one and a half times higher than the rate of 7.3 per cent for NSW overall. In 2000, the highest rates of prematurity were in the Mid Western (18.5 per cent) and Western Sydney Areas (15.3 per cent) (Table 42).

\section{TABLE 41}

GESTATIONAL AGE OF ABORIGINAL AND TORRES STRAIT ISLANDER BABIES, NSW 1996-2000

\begin{tabular}{|c|c|c|c|c|c|c|c|c|c|c|}
\hline \multirow[t]{2}{*}{$\begin{array}{l}\text { Gestational age } \\
\text { (weeks) }\end{array}$} & \multicolumn{2}{|c|}{1996} & \multicolumn{2}{|c|}{1997} & \multicolumn{2}{|c|}{$\begin{array}{c}\text { Year } \\
1998\end{array}$} & \multicolumn{2}{|c|}{1999} & \multicolumn{2}{|c|}{2000} \\
\hline & No. & $\%$ & No. & $\%$ & No. & $\%$ & No. & $\%$ & No. & $\%$ \\
\hline $20-27$ & 25 & 1.5 & 29 & 1.6 & 26 & 1.3 & 18 & 0.9 & 33 & 1.6 \\
\hline 28-31 & 17 & 1.0 & 18 & 1.0 & 26 & 1.3 & 29 & 1.4 & 29 & 1.4 \\
\hline $32-36$ & 139 & 8.1 & 182 & 9.8 & 167 & 8.1 & 209 & 10.1 & 185 & 8.7 \\
\hline $37-41$ & 1508 & 87.5 & 1584 & 85.4 & 1822 & 88.1 & 1780 & 85.7 & 1839 & 86.7 \\
\hline $42+$ & 33 & 1.9 & 40 & 2.2 & 27 & 1.3 & 42 & 2.0 & 36 & 1.7 \\
\hline Not stated & 2 & 0.1 & 1 & 0.1 & 0 & 0.0 & 0 & 0.0 & 0 & 0.0 \\
\hline TOTAL & 1724 & 100.0 & 1854 & 100.0 & 2068 & 100.0 & 2078 & 100.0 & 2122 & 100.0 \\
\hline
\end{tabular}

Source: NSW Midwives Data Collection (HOIST). Epidemiology and Surveillance Branch, NSW Department of Health.

\section{TABLE 42}

GESTATIONAL AGE OF ABORIGINAL AND TORRES STRAIT ISLANDER BABIES BY HEALTH AREA OF RESIDENCE, NSW 2000

\begin{tabular}{|c|c|c|c|c|c|c|}
\hline \multirow[t]{3}{*}{ Health Area } & \multirow{2}{*}{\multicolumn{6}{|c|}{ Gestational age (weeks) }} \\
\hline & Less than 37 & & & & \multicolumn{2}{|c|}{ TOTAL } \\
\hline & No. & $\%$ & No. & $\%$ & No. & $\%$ \\
\hline Central Sydney & 8 & 11.6 & 61 & 88.4 & 69 & 100.0 \\
\hline Northern Sydney ${ }^{\#}$ & - & - & - & - & 9 & 100.0 \\
\hline Western Sydney & 21 & 15.3 & 116 & 84.7 & 137 & 100.0 \\
\hline Wentworth & 7 & 10.8 & 58 & 89.2 & 65 & 100.0 \\
\hline South Western Sydney & 14 & 14.1 & 85 & 85.9 & 99 & 100.0 \\
\hline Central Coast & 11 & 15.1 & 62 & 84.9 & 73 & 100.0 \\
\hline Hunter & 22 & 14.0 & 135 & 86.0 & 157 & 100.0 \\
\hline Illawarra & 18 & 13.0 & 120 & 87.0 & 138 & 100.0 \\
\hline South Eastern Sydney & 5 & 13.9 & 31 & 86.1 & 36 & 100.0 \\
\hline Northern Rivers & 22 & 12.5 & 154 & 87.5 & 176 & 100.0 \\
\hline Mid North Coast & 16 & 7.3 & 203 & 92.7 & 219 & 100.0 \\
\hline New England & 27 & 10.5 & 230 & 89.5 & 257 & 100.0 \\
\hline Macquarie & 18 & 8.1 & 205 & 91.9 & 223 & 100.0 \\
\hline Mid Western & 23 & 18.5 & 101 & 81.5 & 124 & 100.0 \\
\hline Far West & 14 & 9.7 & 130 & 90.3 & 144 & 100.0 \\
\hline Greater Murray & 12 & 10.8 & 99 & 89.2 & 111 & 100.0 \\
\hline Southern & 7 & 10.1 & 62 & 89.9 & 69 & 100.0 \\
\hline Other-Not stated & 1 & 6.3 & 15 & 93.8 & 16 & 100.0 \\
\hline TOTAL & 247 & 11.6 & 1875 & 88.4 & 2122 & 100.0 \\
\hline
\end{tabular}

Source: NSW Midwives Data Collection (HOIST). Epidemiology and Surveillance Branch, NSW Department of Health.

\# Information not shown for Health Areas where the number of babies is less than five in a group. 


\subsection{APGAR SCORE}

In 2000, 3.2 per cent of Aboriginal and Torres Strait Islander babies were born with an Apgar score less than seven (Table 43). This rate is slightly higher than the rate of 2.3 per cent for NSW overall (Section 1.15, page 27).

\section{TABLE 43}

APGAR SCORE OF ABORIGINAL AND TORRES STRAIT ISLANDER BABIES, NSW 1996-2000

\begin{tabular}{|c|c|c|c|c|c|c|c|c|c|c|}
\hline \multirow[t]{2}{*}{$\begin{array}{l}\text { Apgar score } \\
\text { at } 5 \text { minutes }\end{array}$} & \multicolumn{4}{|c|}{1996} & \multicolumn{2}{|c|}{$\begin{array}{l}\text { Year } \\
1998\end{array}$} & \multicolumn{2}{|c|}{1999} & \multicolumn{2}{|c|}{2000} \\
\hline & No. & $\%$ & No. & $\%$ & No. & $\%$ & No. & $\%$ & No. & $\%$ \\
\hline $0-4$ & 33 & 1.9 & 42 & 2.3 & 38 & 1.8 & 36 & 1.7 & 41 & 1.9 \\
\hline $5-6$ & 34 & 2.0 & 34 & 1.8 & 28 & 1.4 & 24 & 1.2 & 26 & 1.2 \\
\hline $7+$ & 1652 & 95.8 & 1770 & 95.5 & 1989 & 96.2 & 2003 & 96.4 & 2045 & 96.4 \\
\hline Not stated & 5 & 0.3 & 8 & 0.4 & 13 & 0.6 & 15 & 0.7 & 10 & 0.5 \\
\hline TOTAL & 1724 & 100.0 & 1854 & 100.0 & 2068 & 100.0 & 2078 & 100.0 & 2122 & 100.0 \\
\hline
\end{tabular}

Source: NSW Midwives Data Collection (HOIST). Epidemiology and Surveillance Branch, NSW Department of Health.

\subsection{PERINATAL MORTALITY}

Since 1996, the perinatal mortality rate among Aboriginal and Torres Strait Islander babies has varied from 14.0 to 20.0 per 1,000 births (Table 44). The rate of 17.9 per 1,000 in 2000 is almost twice the rate of 9.7 per 1,000 for NSW overall (Section 1.16, page 27).

\section{TABLE 44}

PERINATAL DEATHS AMONG ABORIGINAL AND TORRES STRAIT ISLANDER BABIES, NSW 1996-2000\#

\begin{tabular}{|c|c|c|c|c|c|c|c|c|c|c|}
\hline \multirow[t]{3}{*}{ Perinatal deaths } & \multirow{2}{*}{\multicolumn{2}{|c|}{1996}} & \multirow{2}{*}{\multicolumn{2}{|c|}{1997}} & \multicolumn{2}{|c|}{ Year } & \multirow{2}{*}{\multicolumn{2}{|c|}{1999}} & \multirow{2}{*}{\multicolumn{2}{|c|}{2000}} \\
\hline & & & & & & & & & & \\
\hline & No. & 1,000 & No. & 1,000 & No. & 1,000 & No. & 1,000 & No. & 1,000 \\
\hline Stillbirth & 20 & 11.6 & 24 & 12.9 & 21 & 10.2 & 21 & 10.1 & 24 & 11.3 \\
\hline Neonatal death & 10 & 5.8 & 13 & 7.0 & 11 & 5.3 & 8 & 3.8 & 14 & 6.6 \\
\hline TOTAL PERINATAL DEATHS & 30 & 17.4 & 37 & 20.0 & 32 & 15.5 & 29 & 14.0 & 38 & 17.9 \\
\hline
\end{tabular}

Source: NSW Midwives Data Collection (HOIST). Epidemiology and Surveillance Branch, NSW Department of Health.

\# Perinatal deaths include deaths reported to the MDC only. As the MDC form is completed at discharge or transfer of the baby, deaths occurring after this time may not be reported to the MDC. 


\section{PART 4: MATERNAL COUNTRY OF BIRTH}

In this section maternal countries of birth are combined into English-speaking and other regional groups. The country groups and individual countries are listed in Appendix 3. Recent trends in confinements for individual maternal countries of birth are shown in Table 4 (page 20).

\subsection{TRENDS IN CONFINEMENTS}

Between 1996 and 2000, about 20 per cent of mothers were born in non-English speaking countries (Table 45). The proportion of mothers from Asian countries increased slightly from 10.5 to 11.6 per cent, while the proportion of mothers from southern European countries continued to decrease slightly from 1.8 to 1.4 per cent.

\section{TABLE 45}

CONFINEMENTS AND BIRTHS BY COUNTRY OF BIRTH GROUP, NSW 1996-2000

\begin{tabular}{|c|c|c|c|c|c|c|c|c|c|c|}
\hline \multirow[t]{2}{*}{ Plurality } & \multicolumn{2}{|c|}{1996} & \multicolumn{2}{|c|}{1997} & \multicolumn{2}{|c|}{$\begin{array}{l}\text { Year } \\
1998\end{array}$} & \multicolumn{2}{|c|}{1999} & \multicolumn{2}{|c|}{2000} \\
\hline & No. & $\%$ & No. & $\%$ & No. & $\%$ & No. & $\%$ & No. & $\%$ \\
\hline \multicolumn{11}{|l|}{ Confinements } \\
\hline English speaking & 67889 & 79.6 & 68827 & 79.2 & 67971 & 79.9 & 68381 & 79.5 & 68105 & 78.8 \\
\hline \multicolumn{10}{|l|}{ Melanesia, Micronesia \& } & 0.8 \\
\hline Polynesia & 1410 & 1.7 & 1561 & 1.8 & 1590 & 1.9 & 1540 & 1.8 & 1606 & 1.9 \\
\hline $\begin{array}{l}\text { Southern Europe } \\
\text { Western \& Northern }\end{array}$ & 1530 & 1.8 & 1516 & 1.7 & 1380 & 1.6 & 1337 & 1.6 & 1217 & 1.4 \\
\hline Europe & 614 & 0.7 & 627 & 0.7 & 646 & 0.8 & 690 & 0.8 & 671 & 0.8 \\
\hline \multicolumn{11}{|l|}{$\begin{array}{l}\text { Eastern Europe, } \\
\text { Russia,Central Asian }\end{array}$} \\
\hline \& Baltic States & 364 & 0.4 & 393 & 0.5 & 362 & 0.4 & 421 & 0.5 & 428 & 0.5 \\
\hline Middle East \& Africa & 3676 & 4.3 & 3793 & 4.4 & 3670 & 4.3 & 3579 & 4.2 & 3685 & 4.3 \\
\hline South East Asia & 4587 & 5.4 & 4599 & 5.3 & 4157 & 4.9 & 4659 & 5.4 & 5085 & 5.9 \\
\hline North East Asia & 3226 & 3.8 & 3325 & 3.8 & 3097 & 3.6 & 3225 & 3.8 & 3449 & 4.0 \\
\hline Southern Asia & 1170 & 1.4 & 1407 & 1.6 & 1349 & 1.6 & 1398 & 1.6 & 1476 & 1.7 \\
\hline Other-Not stated & 80 & 0.1 & 185 & 0.2 & 167 & 0.2 & 12 & 0.0 & 30 & 0.0 \\
\hline TOTAL & 85302 & 100.0 & 86920 & 100.0 & 85072 & 100.0 & 85967 & 100.0 & 86460 & 100.0 \\
\hline \multicolumn{11}{|l|}{ Births } \\
\hline English speaking & 68816 & 79.6 & 69843 & 79.2 & 69008 & 80.0 & 69460 & 79.6 & 69300 & 78.8 \\
\hline $\begin{array}{l}\text { Central \& South America } \\
\text { Melanesia, Micronesia \& }\end{array}$ & 761 & 0.9 & 693 & 0.8 & 689 & 0.8 & 730 & 0.8 & 716 & 0.8 \\
\hline Polynesia & 1431 & 1.7 & 1585 & 1.8 & 1610 & 1.9 & 1555 & 1.8 & 1636 & 1.9 \\
\hline $\begin{array}{l}\text { Southern Europe } \\
\text { Western \& Northern }\end{array}$ & 1559 & 1.8 & 1532 & 1.7 & 1412 & 1.6 & 1361 & 1.6 & 1256 & 1.4 \\
\hline $\begin{array}{l}\text { Europe } \\
\text { Eastern Europe. }\end{array}$ & 625 & 0.7 & 633 & 0.7 & 652 & 0.8 & 710 & 0.8 & 688 & 0.8 \\
\hline $\begin{array}{l}\text { Russia, Central Asian } \\
\text { \& Baltic States }\end{array}$ & 366 & 0.4 & 398 & 0.5 & 365 & 0.4 & 423 & 0.5 & 439 & 0.5 \\
\hline Middle East \& Africa & 3722 & 4.3 & 3854 & 4.4 & 3731 & 4.3 & 3644 & 4.2 & 3747 & 4.3 \\
\hline South East Asia & 4624 & 5.4 & 4636 & 5.3 & 4181 & 4.8 & 4707 & 5.4 & 5127 & 5.8 \\
\hline North East Asia & 3260 & 3.8 & 3355 & 3.8 & 3118 & 3.6 & 3266 & 3.7 & 3483 & 4.0 \\
\hline Southern Asia & 1182 & 1.4 & 1416 & 1.6 & 1360 & 1.6 & 1420 & 1.6 & 1499 & 1.7 \\
\hline Other-Not stated & 83 & 0.1 & 188 & 0.2 & 179 & 0.2 & 13 & 0.0 & 31 & 0.0 \\
\hline TOTAL & 86429 & 100.0 & 88133 & 100.0 & 86305 & 100.0 & 87289 & 100.0 & 87922 & 100.0 \\
\hline
\end{tabular}

Source: NSW Midwives Data Collection (HOIST). Epidemiology and Surveillance Branch, NSW Department of Health. 


\subsection{MATERNAL AGE}

Births to teenage mothers were more common among mothers born in English-speaking countries than nonEnglish speaking countries (Table 46, Figure 5), while the largest proportions of mothers aged 35 years and over were born in North East Asia (33.4 per cent) and Western and Northern Europe (30.3 per cent).

\begin{tabular}{|c|c|c|c|c|c|c|c|c|c|c|}
\hline \multirow[t]{3}{*}{ Country of birth group } & \multicolumn{10}{|c|}{ Maternal age (years) } \\
\hline & \multicolumn{2}{|c|}{$12-19$} & \multicolumn{2}{|c|}{$20-34$} & \multicolumn{2}{|c|}{$35+$} & \multicolumn{2}{|c|}{ Not stated } & \multicolumn{2}{|c|}{ TOTAL } \\
\hline & No. & $\%$ & No. & $\%$ & No. & $\%$ & No. & $\%$ & No. & $\%$ \\
\hline English speaking & 3565 & 5.2 & 53481 & 78.5 & 11038 & 16.2 & 21 & 0.0 & 68105 & 100.0 \\
\hline Central \& South America & 14 & 2.0 & 506 & 71.5 & 188 & 26.6 & 0 & 0.0 & 708 & 100.0 \\
\hline Melanesia, Micronesia \& Polynesia & 44 & 2.7 & 1247 & 77.6 & 315 & 19.6 & 0 & 0.0 & 1606 & 100.0 \\
\hline Southern Europe & 14 & 1.2 & 886 & 72.8 & 317 & 26.0 & 0 & 0.0 & 1217 & 100.0 \\
\hline $\begin{array}{l}\text { Western \& Northern Europe } \\
\text { Eastern Europe, Russia, Central }\end{array}$ & 0 & 0.0 & 467 & 69.6 & 203 & 30.3 & 1 & 0.1 & 671 & 100.0 \\
\hline Asian \& Baltic States & 8 & 1.9 & 330 & 77.1 & 90 & 21.0 & 0 & 0.0 & 428 & 100.0 \\
\hline Middle East \& Africa & 102 & 2.8 & 2915 & 79.1 & 668 & 18.1 & 0 & 0.0 & 3685 & 100.0 \\
\hline South East Asia & 87 & 1.7 & 3925 & 77.2 & 1072 & 21.1 & 1 & 0.0 & 5085 & 100.0 \\
\hline North East Asia & 11 & 0.3 & 2286 & 66.3 & 1152 & 33.4 & 0 & 0.0 & 3449 & 100.0 \\
\hline Southern Asia & 8 & 0.5 & 1182 & 80.1 & 286 & 19.4 & 0 & 0.0 & 1476 & 100.0 \\
\hline Other-Not stated & 0 & 0.0 & 24 & 80.0 & 5 & 16.7 & 1 & 3.3 & 30 & 100.0 \\
\hline TOTAL & 3853 & 4.5 & 67249 & 77.8 & 15334 & 17.7 & 24 & 0.0 & 86460 & 100.0 \\
\hline
\end{tabular}

Source: NSW Midwives Data Collection (HOIST). Epidemiology and Surveillance Branch, NSW Department of Health.

\section{FIGURE 5}

AGE OF MOTHER BY COUNTRY OF BIRTH GROUP, NSW 2000

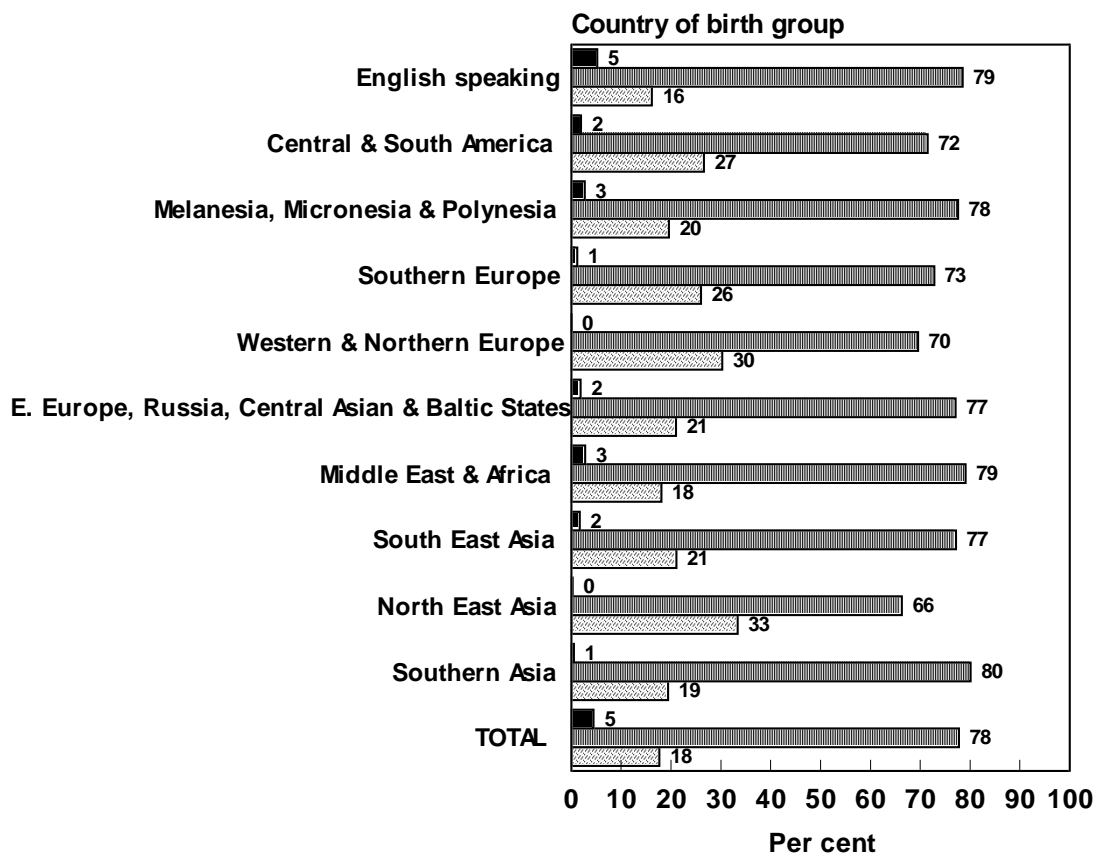

Less than 20 years 血 20-34 years 橉 35+ years

Source: NSW Midwives Data Collection (HOIST). Epidemiology and Surveillance Branch, NSW Department of Health. 


\subsection{HEALTH AREA OF RESIDENCE}

In 2000, the proportion of mothers born in non-English speaking countries was highest in the Central Sydney Area (44.7 per cent), followed by the South Western Sydney and Western Sydney Areas (40.3 and 36.0 per cent respectively). Six per cent of mothers were born in South East Asian countries, 41.9 per cent of whom were resident in the South Western Sydney Area. Four per cent of mothers were born in Middle Eastern or African countries and 61.3 per cent of these mothers were resident in the South Western or Western Sydney Areas. A further 4.0 per cent of mothers were born in North East Asian countries, the majority living in the Central Sydney, South Eastern Sydney, Northern Sydney or Western Sydney Areas (Table 47).

\section{TABLE 47}

HEALTH AREA OF RESIDENCE BY MATERNAL COUNTRY OF BIRTH GROUP, NSW 2000\#

Health Area

English Country of birth group

Central Melanesia Southern Western Eastern Middle South North Southern Other- TOTAL

South \& $\quad$ Northern Russia, \&

America Polynesia Europe Central Africa

East East Asia Not

Asian \&

stated

Baltic

States

No. $\%$ No. $\%$ No. $\%$ No. $\%$ No. $\%$ No. $\%$ No. $\%$ No. $\%$ No. $\%$ No. $\%$ No. $\%$ No. $\%$

Central

Sydney
Northern

\begin{tabular}{lllllllllllllllllllllllllllll} 
& Sydney \\
\hline & 7367 & 78.1 & 89 & 0.9 & 111 & 12 & 102 & 1.1 & 189 & 2.0 & 71 & 0.8 & 227 & 2.4 & 381 & 4.0 & 685 & 7.3 & 209 & 22 & 1 & 0.0 & 9432 & 100.0
\end{tabular}

Western

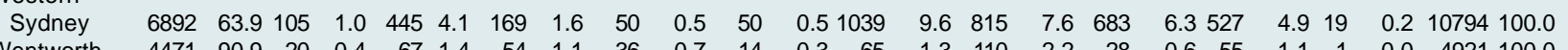

Wentworth

South

Western

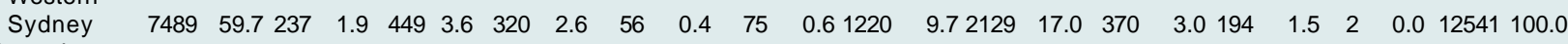

Central

Coast

$\begin{array}{llllllllllllllllllllllll}3632 & 96.3 & 12 & 0.3 & 13 & 0.3 & 7 & 0.2 & 25 & 0.7 & 7 & 0.2 & 7 & 0.2 & 42 & 1.1 & 20 & 0.5 & 6 & 0.2 & 1 & 0.0 & 3772 & 100.0\end{array}$

Hunter

$\begin{array}{lllllllllllllllllllllllll}6741 & 96.6 & 12 & 0.2 & 33 & 0.5 & 23 & 0.3 & 16 & 0.2 & 7 & 0.1 & 16 & 0.2 & 84 & 1.2 & 38 & 0.5 & 11 & 0.2 & 0 & 0.0 & 6981 & 100.0\end{array}$

Illawarra

$\begin{array}{lllllllllllllllllllllllll}4074 & 92.4 & 33 & 0.7 & 30 & 0.7 & 98 & 2.2 & 35 & 0.8 & - & - & 40 & 0.9 & 61 & 1.4 & 25 & 0.6 & 7 & 0.2 & - & - & 4407 & 100.0\end{array}$

South

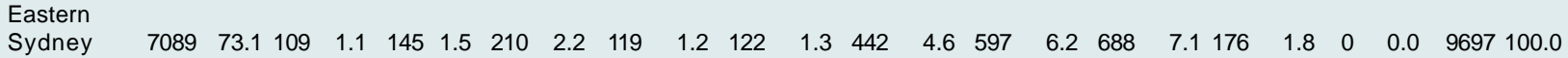

Northern

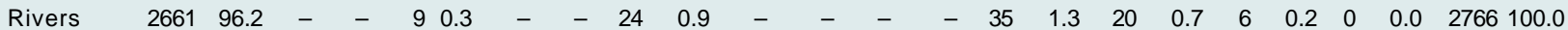

Mid North

Coast

New

$\begin{array}{llllllllllllllllllllllllll}2718 & 97.0 & 5 & 0.2 & - & - & - & - & 9 & 0.3 & 6 & 0.2 & - & - & 25 & 0.9 & 16 & 0.6 & 13 & 0.5 & 1 & 0.0 & 2802 & 100.0\end{array}$

$\begin{array}{lllllllllllllllllllllllllll} & \text { England } & 2211 & 97.6 & - & - & 8 & 0.4 & - & - & 7 & 0.3 & 0 & 0.0 & 6 & 0.3 & 17 & 0.8 & 9 & 0.4 & - & - & 1 & 0.0 & 2265 & 100.0\end{array}$

$\begin{array}{llllllllllllllllllllllllllllll}\text { Macquarie } & 1562 & 98.2 & - & - & - & - & - & - & - & - & - & - & - & - & 5 & 0.3 & 6 & 0.4 & - & - & 0 & 0.0 & 1590 & 100.0\end{array}$

$\begin{array}{llllllllllllllllllllllllllllll}\text { Mid Western } & 2219 & 98.0 & 0 & 0.0 & - & - & - & - & 7 & 0.3 & - & - & - & - & 14 & 0.6 & 6 & 0.3 & 5 & 0.2 & 0 & 0.0 & 2264 & 100.0\end{array}$

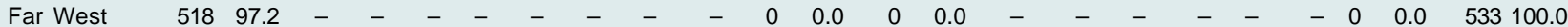

Greater

$\begin{array}{rrrllllllllllllllllllllllll}\text { Murray } & 2421 & 96.2 & - & - & 28 & 1.1 & - & - & 5 & 0.2 & - & - & 7 & 0.3 & 13 & 0.5 & 14 & 0.6 & 22 & 0.9 & 0 & 0.0 & 2517 & 100.0 \\ \text { Southern } & 1697 & 96.1 & - & - & 10 & 0.6 & 7 & 0.4 & 13 & 0.7 & - & - & 9 & 0.5 & 15 & 0.8 & 8 & 0.5 & - & - & 0 & 0.0 & 1766 & 100.0\end{array}$

Other-

$\begin{array}{lllllllllllllllllllllllllllllllll}\text { Not stated } & 598 & 93.9 & 1 & 0.2 & 3 & 0.5 & 3 & 0.5 & 3 & 0.5 & 1 & 0.2 & 5 & 0.8 & - & - & - & - & 0 & 0.0 & - & - & 637 & 100.0\end{array}$

$\begin{array}{lrrrrrrrrrrrrrrrrrrrrrr}\text { TOTAL } & 68105 & 78.8 & 708 & 0.8 & 1606 & 1.9 & 1217 & 1.4 & 671 & 0.8 & 428 & 0.53685 & 4.3 & 5085 & 5.9 & 3449 & 4.01476 & 1.7 & 30 & 0.0 & 86460 & 100.0\end{array}$

Source: NSW Midwives Data Collection (HOIST). Epidemiology and Surveillance Branch, NSW Department of Health.

\# Data not shown for country of birth groups with less than five in a group. 


\subsection{BOOKING STATUS}

In 2000, 97.9 per cent of all mothers were booked at the hospital of birth. The lowest rate (94.5 per cent) was in mothers born in Melanesia, Micronesia and Polynesia. This compared with 97.7 per cent of mothers born in English speaking countries and over 98 per cent of mothers in other country of birth groups.

\subsection{DURATION OF PREGNANCY AT FIRST ANTENATAL VISIT}

In $2000,86.5$ per cent of all mothers commenced antenatal care before 20 weeks gestation. There was some variation between country of birth groups, with 89.0 per cent of mothers born in English speaking countries commencing antenatal care before 20 weeks gestation, compared with 55.9 per cent of mothers born in Melanesia, Micronesia and Polynesia and 67.8 per cent of mothers born in the Middle East and Africa (Table 48).

\section{TABLE 48}

CONFINEMENTS BY COUNTRY OF BIRTH GROUP AND DURATION OF PREGNANCY AT FIRST ANTENATAL VISIT, NSW 2000

\begin{tabular}{|c|c|c|c|c|c|c|c|c|}
\hline \multirow[t]{3}{*}{ Country of birth group } & \multicolumn{8}{|c|}{ Duration of pregnancy at first antenatal visit (weeks) } \\
\hline & \multicolumn{2}{|c|}{ 0-19 } & \multicolumn{2}{|c|}{$20+$} & \multicolumn{2}{|c|}{ Not stated } & \multicolumn{2}{|c|}{ TOTAL } \\
\hline & No. & $\%$ & No. & $\%$ & No. & $\%$ & No. & $\%$ \\
\hline English speaking & 60647 & 89.0 & 6703 & 9.8 & 755 & 1.1 & 68105 & 100.0 \\
\hline Central \& South America & 593 & 83.8 & 112 & 15.8 & 3 & 0.4 & 708 & 100.0 \\
\hline Melanesia, Micronesia \& Polynesia & 897 & 55.9 & 662 & 41.2 & 47 & 2.9 & 1606 & 100.0 \\
\hline Southern Europe & 1053 & 86.5 & 158 & 13.0 & 6 & 0.5 & 1217 & 100.0 \\
\hline Western \& Northern Europe & 593 & 88.4 & 69 & 10.3 & 9 & 1.3 & 671 & 100.0 \\
\hline Eastern Europe, Russia, & & & & & & & & \\
\hline Central Asian \& Baltic States & 343 & 80.1 & 81 & 18.9 & 4 & 0.9 & 428 & 100.0 \\
\hline Middle East \& Africa & 2499 & 67.8 & 1162 & 31.5 & 24 & 0.7 & 3685 & 100.0 \\
\hline South East Asia & 4162 & 81.8 & 884 & 17.4 & 39 & 0.8 & 5085 & 100.0 \\
\hline North East Asia & 2767 & 80.2 & 670 & 19.4 & 12 & 0.3 & 3449 & 100.0 \\
\hline Southern Asia & 1228 & 83.2 & 242 & 16.4 & 6 & 0.4 & 1476 & 100.0 \\
\hline Other-Not stated & 21 & 70.0 & 5 & 16.7 & 4 & 13.3 & 30 & 100.0 \\
\hline TOTAL & 74803 & 86.5 & 10748 & 12.4 & 909 & 1.1 & 86460 & 100.0 \\
\hline
\end{tabular}

Source: NSW Midwives Data Collection (HOIST). Epidemiology and Surveillance Branch, NSW Department of Health.

\subsection{SMOKING IN PREGNANCY}

In 2000, smoking at any time during pregnancy was far more comon among mothers born in English speaking countries than mothers born in non-English speaking countries (Table 49). About one in five mothers born in English speaking countries smoked at some time during pregnancy, followed by one in six mothers born in Southern European countries and less than one in ten mothers born in other non-English speaking countries.
Smoking in the second half of pregnancy poses the greatest risk to the health of both mother and baby. Mothers born in Central and South America and South East Asia were more likely to quit smoking in the second half of pregnancy compared to mothers born in other country of birth groups (Table 50).

\section{TABLE 49}

CONFINEMENTS BY COUNTRY OF BIRTH GROUP AND SMOKING IN PREGNANCY, NSW 2000

\begin{tabular}{|c|c|c|c|c|c|c|c|c|}
\hline \multirow{3}{*}{ Country of birth group } & \multicolumn{8}{|c|}{ Smoking in pregnancy } \\
\hline & \multicolumn{2}{|c|}{ No } & \multicolumn{2}{|c|}{ Yes } & \multicolumn{2}{|c|}{ Not stated } & \multicolumn{2}{|c|}{ TOTAL } \\
\hline & No. & $\%$ & No. & $\%$ & No. & $\%$ & No. & $\%$ \\
\hline English speaking & 54055 & 79.4 & 14032 & 20.6 & 18 & 0.0 & 68105 & 100.0 \\
\hline Central \& South America & 669 & 94.5 & 39 & 5.5 & 0 & 0.0 & 708 & 100.0 \\
\hline Melanesia, Micronesia \& Polynesia & 1458 & 90.8 & 148 & 9.2 & 0 & 0.0 & 1606 & 100.0 \\
\hline Southern Europe & 1038 & 85.3 & 179 & 14.7 & 0 & 0.0 & 1217 & 100.0 \\
\hline Western \& Northern Europe & 609 & 90.8 & 62 & 9.2 & 0 & 0.0 & 671 & 100.0 \\
\hline Eastern Europe, Russia, & & & & & & & & \\
\hline Central Asian \& Baltic States & 386 & 90.2 & 42 & 9.8 & 0 & 0.0 & 428 & 100.0 \\
\hline Middle East \& Africa & 3360 & 91.2 & 325 & 8.8 & 0 & 0.0 & 3685 & 100.0 \\
\hline South East Asia & 4967 & 97.7 & 117 & 2.3 & 1 & 0.0 & 5085 & 100.0 \\
\hline North East Asia & 3411 & 98.9 & 38 & 1.1 & 0 & 0.0 & 3449 & 100.0 \\
\hline Southern Asia & 1460 & 98.9 & 16 & 1.1 & 0 & 0.0 & 1476 & 100.0 \\
\hline Other-Not stated & 24 & 80.0 & 3 & 10.0 & 3 & 10.0 & 30 & 100.0 \\
\hline TOTAL & 71437 & 82.6 & 15001 & 17.4 & 22 & 0.0 & 86460 & 100.0 \\
\hline
\end{tabular}




\section{TABLE 50}

MOTHERS WHO SMOKED AT ALL DURING PREGNANCY BY NUMBER OF CIGARETTES SMOKED IN THE SECOND HALF OF PREGNANCY AND COUNTRY OF BIRTH GROUP, NSW 2000

Country of birth group

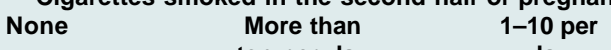

No.

day

\begin{tabular}{|c|c|c|c|c|c|c|c|c|c|}
\hline \multirow[b]{2}{*}{ No. } & \multirow[b]{2}{*}{$\%$} & \multicolumn{2}{|r|}{ day } & \multicolumn{2}{|c|}{ day } & \multicolumn{2}{|c|}{ stated } & \multirow[b]{2}{*}{ No. } & \multirow[b]{2}{*}{$\%$} \\
\hline & & No. & $\%$ & No. & $\%$ & No. & $\%$ & & \\
\hline 571 & 4.1 & 6654 & 47.4 & 6549 & 46.7 & 258 & 1.8 & 14032 & 100.0 \\
\hline 5 & 12.8 & 10 & 25.6 & 23 & 59.0 & 1 & 2.6 & 39 & 100.0 \\
\hline 11 & 7.4 & 43 & 29.1 & 89 & 60.1 & 5 & 3.4 & 148 & 100.0 \\
\hline 3 & 1.7 & 67 & 37.4 & 107 & 59.8 & 2 & 1.1 & 179 & 100.0 \\
\hline 2 & 3.2 & 30 & 48.4 & 29 & 46.8 & 1 & 1.6 & 62 & 100.0 \\
\hline 3 & 7.1 & 18 & 42.9 & 20 & 47.6 & 1 & 2.4 & 42 & 100.0 \\
\hline 10 & 3.1 & 142 & 43.7 & 168 & 51.7 & 5 & 1.5 & 325 & 100.0 \\
\hline 15 & 12.8 & 28 & 23.9 & 66 & 56.4 & 8 & 6.8 & 117 & 100.0 \\
\hline 1 & 2.6 & 7 & 18.4 & 30 & 78.9 & 0 & 0.0 & 38 & 100.0 \\
\hline 1 & 6.3 & 4 & 25.0 & 10 & 62.5 & 1 & 6.3 & 16 & 100.0 \\
\hline 0 & 0.0 & 2 & 66.7 & 1 & 33.3 & 0 & 0.0 & 3 & 100.0 \\
\hline 622 & 4.1 & 7005 & 46.7 & 7092 & 47.3 & 282 & 1.9 & 15001 & 100.0 \\
\hline
\end{tabular}

English speaking

Central \& South America

Melanesia, Micronesia \& Polynesia

Southern Europe

Western \& Northern Europe

Eastern Europe, Russia, Central

Asian \& Baltic States

Middle East \& Africa

South East Asia

North East Asia

Southern Asia

Other-Not stated

TOTAL

Source: NSW Midwives Data Collection (HOIST). Epidemiology and Surveillance Branch, NSW Department of Health.

\subsection{MEDICAL CONDITIONS AND OBSTETRIC COMPLICATIONS}

In 2000, 1.0 per cent mothers born in Melanesia, Micronesia and Polynesia were reported to have diabetes mellitus, three times the rate for all mothers in NSW, though the number of mothers is small. The rates of gestational diabetes in mothers born in Asian countries and Melanesia, Micronesia and Polynesia were over 8 per cent, and were more than twice the rate for all mothers in NSW (Table 51).

\section{TABLE 51}

MATERNAL MEDICAL CONDITIONS AND OBSTETRIC COMPLICATIONS BY COUNTRY OF BIRTH GROUP, NSW 2000

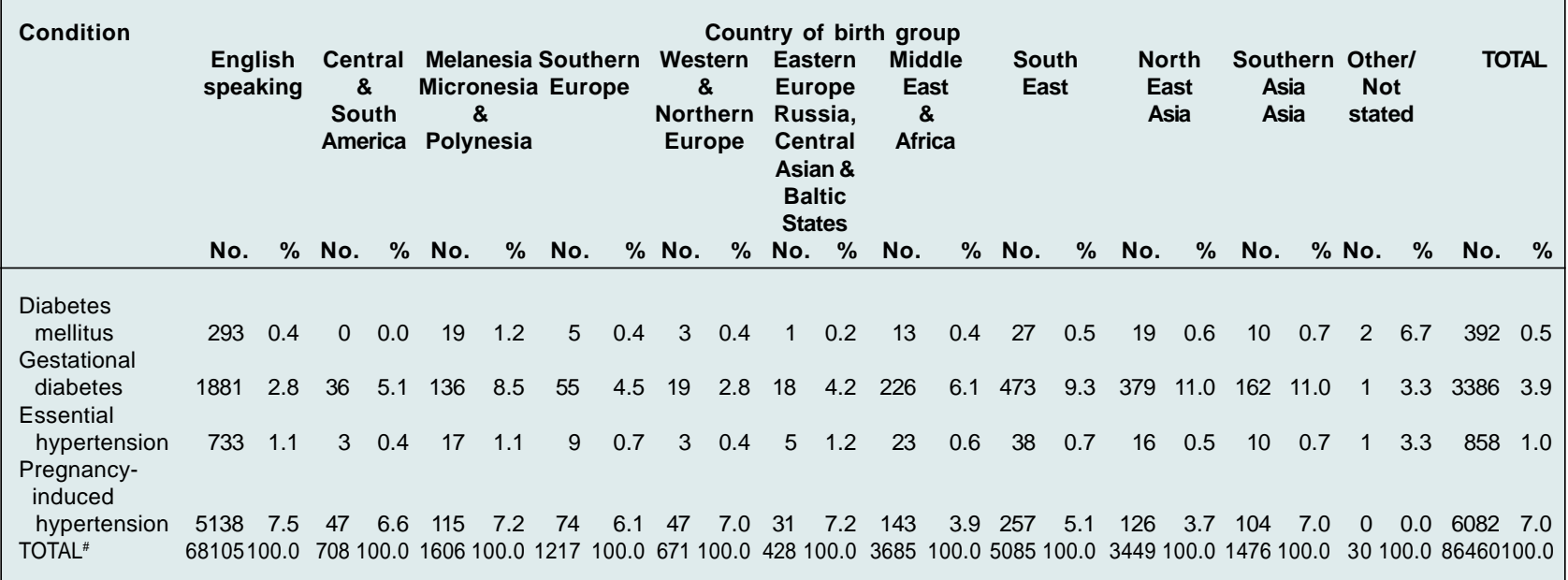

Source: NSW Midwives Data Collection (HOIST). Epidemiology and Surveillance Branch, NSW Department of Health.

\# Total refers to total confinements in NSW. 


\subsection{LABOUR AND DELIVERY}

Mothers born in non-English speaking countries were more likely to have a spontaneous onset of labour than mothers born in English speaking countries and less likely to be induced (Table 52).

Mothers born in Melanesia, Micronesia and Polynesia, and the Middle East and Africa were more likely to have a normal vaginal delivery than mothers in other country of birth groups (Table 53). The highest caesarean section rates were among mothers born in Southern Asia (25.6 per cent) and Central and South America (25.1per cent).

\section{TABLE 52}

LABOUR ONSET BY COUNTRY OF BIRTH GROUP, NSW 2000

\begin{tabular}{|c|c|c|c|c|c|c|c|c|c|c|}
\hline \multirow[t]{2}{*}{$\begin{array}{l}\text { Country of } \\
\text { birth group }\end{array}$} & \multicolumn{2}{|c|}{ Spontaneous } & \multicolumn{2}{|c|}{ No labour ${ }^{\#}$} & \multicolumn{2}{|c|}{$\begin{array}{l}\text { Onset of labour } \\
\text { Induced }\end{array}$} & \multicolumn{2}{|c|}{ Not stated } & \multicolumn{2}{|c|}{ TOTAL } \\
\hline & No. & $\%$ & No. & $\%$ & No. & $\%$ & No. & $\%$ & No. & $\%$ \\
\hline English speaking & 42857 & 62.9 & 8047 & 11.8 & 17193 & 25.2 & 8 & 0.0 & 68105 & 100.0 \\
\hline Central \& South America & 499 & 70.5 & 86 & 12.1 & 123 & 17.4 & 0 & 0.0 & 708 & 100.0 \\
\hline Melanesia, Micronesia \& Polynesia & 1181 & 73.5 & 127 & 7.9 & 298 & 18.6 & 0 & 0.0 & 1606 & 100.0 \\
\hline Southern Europe & 802 & 65.9 & 144 & 11.8 & 271 & 22.3 & 0 & 0.0 & 1217 & 100.0 \\
\hline $\begin{array}{l}\text { Western \& Northern Europe } \\
\text { Eastern Europe, Russia, Central }\end{array}$ & 432 & 64.4 & 83 & 12.4 & 156 & 23.2 & 0 & 0.0 & 671 & 100.0 \\
\hline Asian \& Baltic States & 303 & 70.8 & 39 & 9.1 & 86 & 20.1 & 0 & 0.0 & 428 & 100.0 \\
\hline Middle East \& Africa & 2676 & 72.6 & 337 & 9.1 & 672 & 18.2 & 0 & 0.0 & 3685 & 100.0 \\
\hline South East Asia & 3880 & 76.3 & 444 & 8.7 & 761 & 15.0 & 0 & 0.0 & 5085 & 100.0 \\
\hline North East Asia & 2463 & 71.4 & 439 & 12.7 & 547 & 15.9 & 0 & 0.0 & 3449 & 100.0 \\
\hline Southern Asia & 993 & 67.3 & 178 & 12.1 & 305 & 20.7 & 0 & 0.0 & 1476 & 100.0 \\
\hline Other-Not stated & 20 & 66.7 & 2 & 6.7 & 8 & 26.7 & 0 & 0.0 & 30 & 100.0 \\
\hline TOTAL & 56106 & 64.9 & 9926 & 11.5 & 20420 & 23.6 & 8 & 0.0 & 86460 & 100.0 \\
\hline
\end{tabular}

Source: NSW Midwives Data Collection (HOIST). Epidemiology and Surveillance Branch, NSW Department of Health.

\# No labour indicates elective caesarean section.

\section{TABLE 53}

TYPE OF DELIVERY BY COUNTRY OF BIRTH GROUP, NSW 2000

\begin{tabular}{|c|c|c|c|c|c|c|c|c|c|c|c|c|c|c|c|c|}
\hline \multirow{3}{*}{$\begin{array}{l}\text { Country of } \\
\text { birth group }\end{array}$} & \multirow{2}{*}{\multicolumn{2}{|c|}{$\begin{array}{l}\text { Normal } \\
\text { vaginal }\end{array}$}} & \multirow{2}{*}{\multicolumn{2}{|c|}{ Forceps }} & \multirow{2}{*}{\multicolumn{2}{|c|}{$\begin{array}{l}\text { Vacuum } \\
\text { extraction }\end{array}$}} & \multirow{2}{*}{\multicolumn{4}{|c|}{\begin{tabular}{lc}
\multicolumn{2}{c}{ Type of delivery } \\
Vaginal & Elective \\
breech & caesarean \\
& section
\end{tabular}}} & \multirow{2}{*}{\multicolumn{2}{|c|}{$\begin{array}{l}\text { Emergency } \\
\text { caesarean } \\
\text { section }^{\#}\end{array}$}} & \multirow{2}{*}{\multicolumn{2}{|c|}{$\begin{array}{l}\text { Not } \\
\text { stated }\end{array}$}} & \multirow{2}{*}{\multicolumn{2}{|c|}{ TOTAL }} \\
\hline & & & & & & & & & & & & & & & & \\
\hline & No. & $\%$ & No. & $\%$ & No. & $\%$ & No. & $\%$ & No. & $\%$ & No. & $\%$ & No. & $\%$ & No. & $\%$ \\
\hline $\begin{array}{l}\text { English speaking } \\
\text { Central \& South }\end{array}$ & 45600 & 67.0 & 3076 & 4.5 & 4167 & 6.1 & 515 & 0.8 & 8047 & 11.8 & 6689 & 9.8 & 11 & 0.0 & 68105 & 100.0 \\
\hline $\begin{array}{l}\text { America } \\
\text { Melanesia, Micronesia }\end{array}$ & 453 & 64.0 & 33 & 4.7 & 40 & 5.6 & 4 & 0.6 & 86 & 12.1 & 92 & 13.0 & 0 & 0.0 & 708 & 100.0 \\
\hline $\begin{array}{l}\text { \& Polynesia } \\
\text { Southern Europe } \\
\text { Western \& Northern }\end{array}$ & $\begin{array}{r}1215 \\
835\end{array}$ & $\begin{array}{l}75.7 \\
68.6\end{array}$ & $\begin{array}{l}50 \\
56\end{array}$ & $\begin{array}{l}3.1 \\
4.6\end{array}$ & $\begin{array}{l}63 \\
57\end{array}$ & $\begin{array}{l}3.9 \\
4.7\end{array}$ & $\begin{array}{l}15 \\
13\end{array}$ & $\begin{array}{l}0.9 \\
1.1\end{array}$ & $\begin{array}{l}127 \\
144\end{array}$ & $\begin{array}{r}7.9 \\
11.8\end{array}$ & $\begin{array}{l}136 \\
112\end{array}$ & $\begin{array}{l}8.5 \\
9.2\end{array}$ & $\begin{array}{l}0 \\
0\end{array}$ & $\begin{array}{l}0.0 \\
0.0\end{array}$ & $\begin{array}{l}1606 \\
1217\end{array}$ & $\begin{array}{l}100.0 \\
100.0\end{array}$ \\
\hline $\begin{array}{l}\text { Europe } \\
\text { Eastern Europe, Russia } \\
\text { Central Asian \& Baltic }\end{array}$ & $a{ }^{425}$ & 63.3 & 36 & 5.4 & 51 & 7.6 & 7 & 1.0 & 83 & 12.4 & 69 & 10.3 & 0 & 0.0 & 671 & 100.0 \\
\hline $\begin{array}{l}\text { States } \\
\text { Middle East \& Africa }\end{array}$ & $\begin{array}{r}288 \\
2783\end{array}$ & $\begin{array}{l}67.3 \\
75.5\end{array}$ & $\begin{array}{r}24 \\
106\end{array}$ & $\begin{array}{l}5.6 \\
2.9\end{array}$ & $\begin{array}{r}40 \\
168\end{array}$ & $\begin{array}{l}9.3 \\
4.6\end{array}$ & $\begin{array}{r}1 \\
36\end{array}$ & $\begin{array}{l}0.2 \\
1.0\end{array}$ & $\begin{array}{r}39 \\
337\end{array}$ & $\begin{array}{l}9.1 \\
9.1\end{array}$ & $\begin{array}{r}36 \\
254\end{array}$ & $\begin{array}{l}8.4 \\
6.9\end{array}$ & $\begin{array}{l}0 \\
1\end{array}$ & $\begin{array}{l}0.0 \\
0.0\end{array}$ & $\begin{array}{r}428 \\
3685\end{array}$ & $\begin{array}{l}100.0 \\
100.0\end{array}$ \\
\hline South East Asia & 3430 & 67.5 & 230 & 4.5 & 359 & 7.1 & 53 & 1.0 & 444 & 8.7 & 568 & 11.2 & 1 & 0.0 & 5085 & 100.0 \\
\hline North East Asia & 2117 & 61.4 & 195 & 5.7 & 310 & 9.0 & 14 & 0.4 & 439 & 12.7 & 374 & 10.8 & 0 & 0.0 & 3449 & 100.0 \\
\hline Southern Asia & 877 & 59.4 & 96 & 6.5 & 112 & 7.6 & 11 & 0.7 & 178 & 12.1 & 200 & 13.6 & 2 & 0.1 & 1476 & 100.0 \\
\hline Other-Not stated & 26 & 86.7 & 2 & 6.7 & 0 & 0.0 & 0 & 0.0 & 2 & 6.7 & 0 & 0.0 & 0 & 0.0 & 30 & 100.0 \\
\hline TOTAL & 58049 & 67.1 & 3904 & 4.5 & 5367 & 6.2 & 669 & 0.8 & 9926 & 11.5 & 8530 & 9.9 & 15 & 0.0 & 86460 & 100.0 \\
\hline
\end{tabular}

Source: NSW Midwives Data Collection (HOIST). Epidemiology and Surveillance Branch, NSW Department of Health.

\# Emergency caesarean section includes caesarean sections where the onset of labour was not stated. 


\subsection{BIRTHWEIGHT}

The rate of low birthweight (less than 2,500 grams) in 2000 was 6.4 per cent in NSW. The highest rates of low birthweight were in babies of mothers born in Southern Asian countries (9.9 per cent) (Table 54). Babies of mothers born in North East Asia and Central and South America were least likely to be low birthweight.

\section{TABLE 54}

BIRTHWEIGHT BY MATERNAL COUNTRY OF BIRTH GROUP, NSW 2000

\begin{tabular}{|c|c|c|c|c|c|c|c|c|}
\hline \multirow{3}{*}{$\begin{array}{l}\text { Country of } \\
\text { birth group }\end{array}$} & \multicolumn{8}{|c|}{ Birthweight (grams) } \\
\hline & \multicolumn{2}{|c|}{ Less than 2,500} & \multicolumn{2}{|c|}{$2,500+$} & \multicolumn{2}{|c|}{ Not stated } & \multicolumn{2}{|c|}{ TOTAL } \\
\hline & No. & $\%$ & No. & $\%$ & No. & $\%$ & No. & $\%$ \\
\hline English speaking & 4472 & 6.5 & 64789 & 93.5 & 39 & 0.1 & 69300 & 100.0 \\
\hline Central \& South America & 29 & 4.1 & 686 & 95.8 & 1 & 0.1 & 716 & 100.0 \\
\hline Melanesia, Micronesia \& Polynesia & 130 & 7.9 & 1506 & 92.1 & 0 & 0.0 & 1636 & 100.0 \\
\hline Southern Europe & 94 & 7.5 & 1161 & 92.4 & 1 & 0.1 & 1256 & 100.0 \\
\hline Western \& Northern Europe & 52 & 7.6 & 636 & 92.4 & 0 & 0.0 & 688 & 100.0 \\
\hline Eastern Europe, Russia, Central & & & & & & & & \\
\hline Asian \& Baltic States & 32 & 7.3 & 407 & 92.7 & 0 & 0.0 & 439 & 100.0 \\
\hline Middle East \& Africa & 234 & 6.2 & 3512 & 93.7 & 1 & 0.0 & 3747 & 100.0 \\
\hline South East Asia & 323 & 6.3 & 4801 & 93.6 & 3 & 0.1 & 5127 & 100.0 \\
\hline North East Asia & 144 & 4.1 & 3337 & 95.8 & 2 & 0.1 & 3483 & 100.0 \\
\hline Southern Asia & 149 & 9.9 & 1350 & 90.1 & 0 & 0.0 & 1499 & 100.0 \\
\hline Other-Not stated & 2 & 6.5 & 29 & 93.5 & 0 & 0.0 & 31 & 100.0 \\
\hline TOTAL & 5661 & 6.4 & 82214 & 93.5 & 47 & 0.1 & 87922 & 100.0 \\
\hline
\end{tabular}

Source: NSW Midwives Data Collection (HOIST). Epidemiology and Surveillance Branch, NSW Department of Health.

\subsection{GESTATIONAL AGE}

The rate of prematurity (less than 37 weeks gestation) in 2000 was 7.3 per cent in NSW. The highest rates of prematurity were in babies of mothers born in Western and Northern Europe ( 8.9 per cent). Babies of mothers born in North East Asia or Central and South America were least likely to be premature (Table 55).

\section{TABLE 55}

GESTATIONAL AGE BY MATERNAL COUNTRY OF BIRTH GROUP, NSW 2000

\begin{tabular}{|c|c|c|c|c|c|c|c|c|}
\hline \multirow{2}{*}{$\begin{array}{l}\text { Country of } \\
\text { birth group }\end{array}$} & \multicolumn{8}{|c|}{ Gestational age (weeks) } \\
\hline & No. & $\%$ & No. & $\%$ & No. & $\%$ & No. & $\%$ \\
\hline English speaking & 5126 & 7.4 & 64169 & 92.6 & 5 & 0.0 & 69300 & 100.0 \\
\hline Central \& South America & 45 & 6.3 & 671 & 93.7 & 0 & 0.0 & 716 & 100.0 \\
\hline Melanesia, Micronesia \& Polynesia & 143 & 8.7 & 1493 & 91.3 & 0 & 0.0 & 1636 & 100.0 \\
\hline Southern Europe & 98 & 7.8 & 1158 & 92.2 & 0 & 0.0 & 1256 & 100.0 \\
\hline Western \& Northern Europe & 61 & 8.9 & 627 & 91.1 & 0 & 0.0 & 688 & 100.0 \\
\hline Eastern Europe, Russia, Central & & & & & & & & \\
\hline Asian \& Baltic States & 33 & 7.5 & 406 & 92.5 & 0 & 0.0 & 439 & 100.0 \\
\hline Middle East \& Africa & 255 & 6.8 & 3492 & 93.2 & 0 & 0.0 & 3747 & 100.0 \\
\hline South East Asia & 345 & 6.7 & 4782 & 93.3 & 0 & 0.0 & 5127 & 100.0 \\
\hline North East Asia & 176 & 5.1 & 3307 & 94.9 & 0 & 0.0 & 3483 & 100.0 \\
\hline Southern Asia & 113 & 7.5 & 1386 & 92.5 & 0 & 0.0 & 1499 & 100.0 \\
\hline Other-Not stated & 5 & 16.1 & 25 & 80.6 & 1 & 3.2 & 31 & 100.0 \\
\hline TOTAL & 6400 & 7.3 & 81516 & 92.7 & 6 & 0.0 & 87922 & 100.0 \\
\hline
\end{tabular}

Source: NSW Midwives Data Collection (HOIST). Epidemiology and Surveillance Branch, NSW Department of Health. 


\subsection{APGAR SCORE}

In 2000, 2.3 per cent of all babies (including stillborn babies) had an Apgar score of 7 or less at five minutes and 1.2 per cent had a score of less than 4 (Table 56). Low Apgar scores were most common among babies of mothers born in Melanesia, Micronesia and Polynesia (4.2 per cent).

\section{TABLE 56}

BIRTHS BY COUNTRY OF BIRTH GROUP AND APGAR SCORE AT FIVE MINUTES, NSW 2000"

\begin{tabular}{|c|c|c|c|c|c|c|c|c|c|c|}
\hline \multirow[t]{2}{*}{$\begin{array}{l}\text { Country of } \\
\text { birth group }\end{array}$} & \multicolumn{4}{|c|}{$0-4$} & \multicolumn{2}{|c|}{$\begin{array}{c}\text { Apgar score } \\
7+\end{array}$} & \multicolumn{2}{|c|}{ Not stated } & \multicolumn{2}{|c|}{ TOTAL } \\
\hline & No. & $\%$ & No. & $\%$ & No. & $\%$ & No. & $\%$ & No. & $\%$ \\
\hline English speaking & 816 & 1.2 & 761 & 1.1 & 67584 & 97.5 & 139 & 0.2 & 69300 & 100.0 \\
\hline Central \& South America & 4 & 0.6 & 10 & 1.4 & 701 & 97.9 & 1 & 0.1 & 716 & 100.0 \\
\hline Melanesia, Micronesia \& Polynesia & 39 & 2.4 & 29 & 1.8 & 1561 & 95.4 & 7 & 0.4 & 1636 & 100.0 \\
\hline Southern Europe & 14 & 1.1 & 14 & 1.1 & 1228 & 97.8 & 0 & 0.0 & 1256 & 100.0 \\
\hline $\begin{array}{l}\text { Western \& Northern Europe } \\
\text { Eastern Europe, Russia, Central }\end{array}$ & 13 & 1.9 & 6 & 0.9 & 669 & 97.2 & 0 & 0.0 & 688 & 100.0 \\
\hline Asian \& Baltic States & 6 & 1.4 & 2 & 0.5 & 430 & 97.9 & 1 & 0.2 & 439 & 100.0 \\
\hline Middle East \& Africa & 51 & 1.4 & 38 & 1.0 & 3653 & 97.5 & 5 & 0.1 & 3747 & 100.0 \\
\hline South East Asia & 58 & 1.1 & 54 & 1.1 & 5006 & 97.6 & 9 & 0.2 & 5127 & 100.0 \\
\hline North East Asia & 23 & 0.7 & 26 & 0.7 & 3430 & 98.5 & 4 & 0.1 & 3483 & 100.0 \\
\hline Southern Asia & 18 & 1.2 & 16 & 1.1 & 1464 & 97.7 & 1 & 0.1 & 1499 & 100.0 \\
\hline TOTAL & 1043 & 1.2 & 956 & 1.1 & 85756 & 97.5 & 167 & 0.2 & 87922 & 100.0 \\
\hline
\end{tabular}

Source: NSW Midwives Data Collection (HOIST). Epidemiology and Surveillance Branch, NSW Department of Health. \# Births include stillbirths.

\subsection{PERINATAL OUTCOMES}

In 2000, 99 per cent of babies born in NSW and reported to the MDC were born alive and survived until discharge from the hospital of birth (Table 57). Perinatal mortality was highest among babies of mothers born in Melanesia, Micronesia and Polynesia, and Western and Northern Europe.

\section{TABLE 57}

PERINATAL OUTCOMES BY COUNTRY OF BIRTH GROUP, NSW 2000"

\begin{tabular}{|c|c|c|c|c|c|c|c|c|c|c|c|}
\hline \multirow[t]{2}{*}{$\begin{array}{l}\text { Country of } \\
\text { birth group }\end{array}$} & \multicolumn{2}{|c|}{$\begin{array}{l}\text { Liveborn } \\
\text { surviving }\end{array}$} & \multicolumn{2}{|c|}{ Stillborn } & \multicolumn{2}{|c|}{$\begin{array}{c}\text { Perinatal outcome } \\
\text { Neonatal } \\
\text { death }\end{array}$} & \multicolumn{2}{|c|}{ Not stated } & \multicolumn{2}{|c|}{$\begin{array}{c}\text { Total } \\
\text { births }\end{array}$} & \multirow{2}{*}{$\begin{array}{l}\text { Perinatal } \\
\text { mortality } \\
\text { rate } 11,000 \\
\text { births }\end{array}$} \\
\hline & No. & $\%$ & No. & $\%$ & No. & $\%$ & No. & $\%$ & No. & $\%$ & \\
\hline English speaking & 68627 & 99.0 & 473 & 0.7 & 198 & 0.3 & 2 & 0.0 & 69300 & 100.0 & 9.7 \\
\hline $\begin{array}{l}\text { Central \& South America } \\
\text { Melanesia, Micronesia \& }\end{array}$ & 714 & 99.7 & 2 & 0.3 & 0 & 0.0 & 0 & 0.0 & 716 & 100.0 & - \\
\hline Polynesia & 1601 & 97.9 & 22 & 1.3 & 12 & 0.7 & 1 & 0.1 & 1636 & 100.0 & 20.8 \\
\hline Southern Europe & 1242 & 98.9 & 11 & 0.9 & 3 & 0.2 & 0 & 0.0 & 1256 & 100.0 & 11.1 \\
\hline $\begin{array}{l}\text { Western \& Northern Europ } \\
\text { Eastern Europe, Russia, } \\
\text { Central Asian \& Baltic }\end{array}$ & 678 & 98.5 & 6 & 0.9 & 4 & 0.6 & 0 & 0.0 & 688 & 100.0 & 14.5 \\
\hline States & 437 & 99.5 & 0 & 0.0 & 2 & 0.5 & 0 & 0.0 & 439 & 100.0 & - \\
\hline Middle East \& Africa & 3708 & 99.0 & 27 & 0.7 & 12 & 0.3 & 0 & 0.0 & 3747 & 100.0 & 10.4 \\
\hline South East Asia & 5080 & 99.1 & 32 & 0.6 & 14 & 0.3 & 1 & 0.0 & 5127 & 100.0 & 9.0 \\
\hline North East Asia & 3470 & 99.6 & 9 & 0.3 & 4 & 0.1 & 0 & 0.0 & 3483 & 100.0 & 3.7 \\
\hline Southern Asia & 1479 & 98.7 & 12 & 0.8 & 8 & 0.5 & 0 & 0.0 & 1499 & 100.0 & 13.3 \\
\hline Other-Not stated & 30 & 96.8 & 1 & 3.2 & 0 & 0.0 & 0 & 0.0 & 31 & 100.0 & - \\
\hline TOTAL & 87066 & 99.0 & 595 & 0.7 & 257 & 0.3 & 4 & 0.0 & 87922 & 100.0 & 9.7 \\
\hline
\end{tabular}

Source: NSW Midwives Data Collection (HOIST). Epidemiology and Surveillance Branch, Department of Health.

\# Perinatal deaths include deaths reported to the MDC only. As the MDC form is completed at discharge or transfer of the baby, deaths occurring after this time may not be reported to the MDC.

\#\# Perinatal mortality rate not calculated for country of birth groups with less than five perinatal deaths. 
Information on infants admitted to a neonatal intensive care unit was obtained from the Neonatal Intensive Care Units (NICUS) Data Collection, which is described in Part 1 under Data Sources.

\subsection{REGISTRATION RATE}

There were 2,003 infants registered in NICUS in 2000. The most common reasons for registration of an infant were assisted ventilation for four hours or more (71.1 per cent) and gestational age less than 29 weeks (16.8 per cent). Infants generally met more than one of the registration criteria.

The NICUS registration rate in 2000 was 21.8 per 1,000 live births, which has increased slightly each year since 1992 (17.9 per 1,000 live births). Table 58 shows the registration rate according to the mothers' health area of residence. The relatively low registration rates from the health areas adjoining the New South Wales border reflect the fact that some infants are preferentially referred interstate. The registration rate in health areas with low numbers of births should be interpreted with caution. The proportion of mothers in each health area has remained relatively constant since 1992.

Seventy-nine of the 2,003 infants (3.9 per cent) registered in NICUS were born to Aboriginal and/or Torres Strait Islander mothers. There were 2,153 live births to Aboriginal and/or Torres Strait Islander women recorded by the NSW and ACT Midwives Data Collections for 2000. The registration rate for these infants was 36.7 per 1,000 live births and has increased since 1992. Seventy-six of the 1,841 mothers (4.1 per cent) were Aboriginal and Torres Strait Islander, of whom 28 (36.8 per cent) were residents of the Hunter and New England Health Areas (Table 59). Twenty of the 375 mothers (5.3 per cent) of infants less than 29 weeks and/or less than 1,000 grams were Aboriginal and Torres Strait Islander.

\section{TABLE 58}

NICUS REGISTRATIONS BY HEALTH AREA OF RESIDENCE, NSW \& ACT 2000

\begin{tabular}{|c|c|c|c|c|}
\hline \multirow[t]{2}{*}{ Health Area } & \multicolumn{2}{|c|}{$\begin{array}{l}\text { Total NICUS } \\
\text { registrants }\end{array}$} & \multirow{2}{*}{$\begin{array}{c}\text { Total NSW \& ACT } \\
\text { live births } \\
\text { No. }\end{array}$} & \multirow[t]{2}{*}{$\begin{array}{l}\text { Registrants } \\
\text { per } 1,000 \text { live births }\end{array}$} \\
\hline & No. & $\%$ & & \\
\hline Central Sydney & 138 & 6.9 & 6920 & 19.9 \\
\hline Northern Sydney & 155 & 7.7 & 9527 & 16.3 \\
\hline South Eastern Sydney & 181 & 9.0 & 9877 & 18.3 \\
\hline South Western Sydney & 311 & 15.5 & 13095 & 23.7 \\
\hline Wentworth & 96 & 4.8 & 4470 & 21.5 \\
\hline Western Sydney & 253 & 12.6 & 10960 & 23.1 \\
\hline Central Coast & 76 & 3.8 & 3830 & 19.8 \\
\hline Hunter & 251 & 12.5 & 7048 & 35.6 \\
\hline Illawarra & 89 & 4.4 & 4446 & 20.0 \\
\hline Far West & 6 & 0.3 & 523 & 11.5 \\
\hline Greater Murray & 34 & 1.7 & 2551 & 13.3 \\
\hline Macquarie & 40 & 2.0 & 1613 & 24.8 \\
\hline Mid North Coast & 76 & 3.8 & 2835 & 26.8 \\
\hline Mid Western & 63 & 3.1 & 2298 & 27.4 \\
\hline New England & 56 & 2.8 & 2286 & 24.5 \\
\hline Northern Rivers & 11 & 0.5 & 2759 & 4.0 \\
\hline Southern & 61 & 3.0 & 2196 & 27.8 \\
\hline ACT & 96 & 4.8 & 4118 & 23.3 \\
\hline Interstate & 2 & 0.1 & 574 & 3.5 \\
\hline Overseas & 8 & 0.4 & 0 & - \\
\hline Not stated & 0 & 0.0 & 47 & - \\
\hline TOTAL & 2003 & 100.0 & 91973 & 21.8 \\
\hline
\end{tabular}

Source: NICUS Data Collection. NSW Centre for Perinatal Health Services Research. NSW Midwives Data Collection 2000. Epidemiology and Surveillance Branch, NSW Department of Health. ACT Maternal-Perinatal Data Collection, 1999.

\subsection{MATERNAL CHARACTERISTICS}

There were 1,841 mothers of the 2,003 infants registered in NICUS during 2000. Nearly 80 per cent of the mothers were residents of the Sydney, Central Coast, Hunter and Illawarra Health Areas. The distribution of the mothers Health Area of residence for infants less than 29 weeks and/or less than 1,000 grams was similar to those for the whole group. Of the 375 mothers of infants in this group just over half (52.3 per cent) were residents of the South Western Sydney, Western Sydney, Hunter, South Eastern Sydney or Northern Sydney Health Areas.
The age of mothers of NICUS infants ranged from 15 to 53 years with a mean age of 28.9 years. The mean maternal age was similar across all gestational age groups and has remained constant since 1992 . The proportion of mothers aged 35 years or more was 18.3 in 2000 (compared with 13.7 per cent in 1992 to 20.8 per cent in 1999). There were 6.8 per cent of mothers aged less than 20 years (Table 60). The health areas of residence with the highest proportion of teenage mothers were Far West, Macquarie, Mid Western, New England and Southern. 
There were 1,607 mothers (87.3 per cent) who had an antenatal complication. The most common antenatal complications were threatened preterm labour $(836 / 1,841$; 45.4 per cent $)$, fetal distress $(413 / 1,841 ; 22.4$ per cent $)$, hypertensive disease of pregnancy $(348 / 1,841 ; 18.9$ per cent) and antepartum haemorrhage $(317 / 1,841 ; 17.2$ per cent). Antenatal complications were more frequent in mothers delivering at less than than 37 weeks compared with at term. Even so, 54.3 per cent (278/512) of mothers giving birth at 37-41 weeks gestation had an antenatal complication.

In $2000,81.5$ per cent of mothers of infants born at less than 28 weeks received corticosteroids (Figure 6 and Table 61 ). Over three-quarters (87.5 per cent) of mothers of 28-31 week gestation infants received antenatal corticosteroids. The overall proportion of mothers receiving antenatal corticosteroids has increased from 45 per cent in 1992 to 72.5 per cent in 1999 .

\section{TABLE 59}

CONFINEMENTS BY HEALTH AREA OF RESIDENCE AND ABORIGINALITY, NSW \& ACT 2000

\begin{tabular}{|c|c|c|c|c|c|c|}
\hline \multirow[t]{2}{*}{ Health Area } & \multicolumn{2}{|c|}{ Aboriginal } & \multicolumn{2}{|c|}{ Non-Aboriginal } & \multicolumn{2}{|c|}{ TOTAL } \\
\hline & No. & $\%$ & No. & $\%$ & No. & $\%$ \\
\hline Central Sydney & 1 & 0.8 & 124 & 99.2 & 125 & 6.8 \\
\hline Northern Sydney & 1 & 0.7 & 146 & 99.3 & 147 & 8.0 \\
\hline South East Sydney & 2 & 1.2 & 165 & 98.8 & 167 & 9.1 \\
\hline South West Sydney & 2 & 0.7 & 279 & 99.3 & 281 & 15.3 \\
\hline Wentworth & 3 & 3.5 & 82 & 96.5 & 85 & 4.6 \\
\hline Western Sydney & 3 & 1.3 & 229 & 98.7 & 232 & 12.6 \\
\hline Central Coast & 0 & 0.0 & 72 & 100.0 & 72 & 3.9 \\
\hline Hunter & 17 & 7.4 & 214 & 92.6 & 231 & 12.5 \\
\hline Illawarra & 3 & 3.8 & 77 & 96.3 & 80 & 4.3 \\
\hline Far West & 2 & 33.3 & 4 & 66.7 & 6 & 0.3 \\
\hline Greater Murray & 4 & 12.5 & 28 & 87.5 & 32 & 1.7 \\
\hline Macquarie & 5 & 13.2 & 33 & 86.8 & 38 & 2.1 \\
\hline Mid North Coast & 7 & 9.7 & 65 & 90.3 & 72 & 3.9 \\
\hline Mid Western & 5 & 8.6 & 53 & 91.4 & 58 & 3.2 \\
\hline New England & 11 & 20.8 & 42 & 79.2 & 53 & 2.9 \\
\hline Northern Rivers & 0 & 0.0 & 10 & 100.0 & 10 & 0.5 \\
\hline Southern & 9 & 15.8 & 48 & 84.2 & 57 & 3.1 \\
\hline ACT & 1 & 1.2 & 84 & 98.8 & 85 & 4.6 \\
\hline Interstate & 0 & 0.0 & 2 & 100.0 & 2 & 0.1 \\
\hline Overseas & 0 & 0.0 & 8 & 100.0 & 8 & 0.4 \\
\hline TOTAL & 76 & 4.1 & 1765 & 95.9 & 1841 & 100.0 \\
\hline
\end{tabular}

Source: NICUS Data Collection. NSW Centre for Perinatal Health Services Research.

\section{TABLE 60}

CONFINEMENTS BY HEALTH AREA OF RESIDENCE AND MATERNAL AGE, NSW \& ACT 2000

\begin{tabular}{|c|c|c|c|c|c|c|c|c|}
\hline \multirow[t]{3}{*}{ Health Area } & \multicolumn{6}{|c|}{$\begin{array}{c}\text { Maternal age (years) } \\
20-34\end{array}$} & \multirow{2}{*}{\multicolumn{2}{|c|}{ TOTAL }} \\
\hline & & 20 & & & & & & \\
\hline & No. & $\%$ & No. & $\%$ & No. & $\%$ & No. & $\%$ \\
\hline Central Sydney & 8 & 6.4 & 78 & 62.4 & 39 & 31.2 & 125 & 6.8 \\
\hline Northern Sydney & 0 & 0.0 & 104 & 70.7 & 43 & 29.3 & 147 & 8.0 \\
\hline South East Sydney & 2 & 1.2 & 120 & 71.9 & 45 & 26.9 & 167 & 9.1 \\
\hline South West Sydney & 25 & 8.9 & 220 & 78.3 & 36 & 12.8 & 281 & 15.3 \\
\hline Wentworth & 8 & 9.4 & 61 & 71.8 & 16 & 18.8 & 85 & 4.6 \\
\hline Western Sydney & 10 & 4.3 & 173 & 74.6 & 49 & 21.1 & 232 & 12.6 \\
\hline Central Coast & 6 & 8.3 & 58 & 80.6 & 8 & 11.1 & 72 & 3.9 \\
\hline Hunter & 20 & 8.7 & 182 & 78.8 & 29 & 12.6 & 231 & 12.5 \\
\hline Illawarra & 5 & 6.3 & 61 & 76.3 & 14 & 17.5 & 80 & 4.3 \\
\hline Far West & 1 & 16.7 & 5 & 83.3 & 0 & 0.0 & 6 & 0.3 \\
\hline Greater Murray & 3 & 9.4 & 26 & 81.3 & 3 & 9.4 & 32 & 1.7 \\
\hline Macquarie & 8 & 21.1 & 25 & 65.8 & 5 & 13.2 & 38 & 2.1 \\
\hline Mid North Coast & 7 & 9.7 & 58 & 80.6 & 7 & 9.7 & 72 & 3.9 \\
\hline Mid Western & 7 & 12.1 & 41 & 70.7 & 10 & 17.2 & 58 & 3.2 \\
\hline New England & 6 & 11.3 & 43 & 81.1 & 4 & 7.5 & 53 & 2.9 \\
\hline Northern Rivers & 0 & 0.0 & 10 & 100.0 & 0 & 0.0 & 10 & 0.5 \\
\hline Southern & 7 & 12.3 & 43 & 75.4 & 7 & 12.3 & 57 & 3.1 \\
\hline ACT & 3 & 3.5 & 62 & 72.9 & 20 & 23.5 & 85 & 4.6 \\
\hline Interstate & 0 & 0.0 & 1 & 50.0 & 1 & 50.0 & 2 & 0.1 \\
\hline Overseas & 0 & 0.0 & 8 & 100.0 & 0 & 0.0 & 8 & 0.4 \\
\hline TOTAL & 126 & 6.8 & 1379 & 74.9 & 336 & 18.3 & 1841 & 100.0 \\
\hline
\end{tabular}

Source: NICUS Data Collection. NSW Centre for Perinatal Health Services Research. 


\section{FIGURE6}

CONFINEMENTS BY ANTENATAL CORTICOSTEROID ADMINISTRATION AND GESTATIONAL AGE, NSW \& ACT 1996-2000

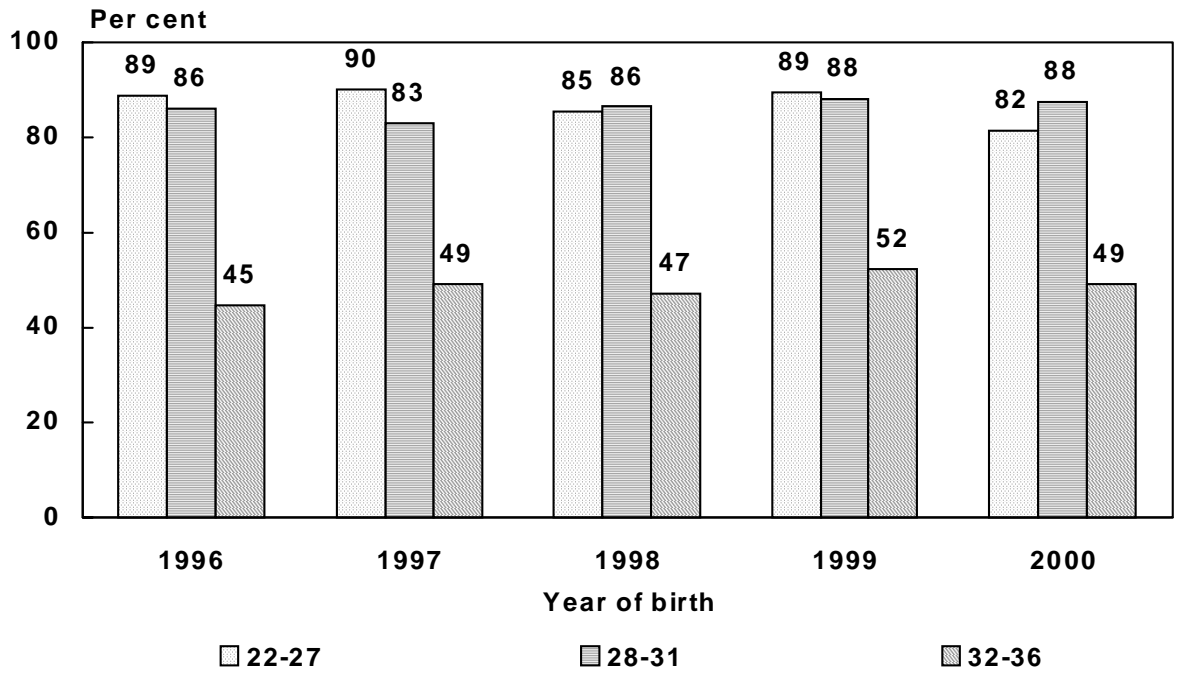

Source: NICUS Data Collection. NSW Centre for Perinatal Health Services Research.

\section{TABLE 61}

CONFINEMENTS BY ANTENATAL CORTICOSTEROID ADMINISTRATION AND GESTATIONAL AGE, NSW \& ACT $1996-2000$

\begin{tabular}{|c|c|c|c|c|c|c|c|c|c|}
\hline \multirow[t]{3}{*}{ Year } & \multirow{3}{*}{$\begin{array}{l}\text { Corticosteroid } \\
\text { administration }\end{array}$} & \multicolumn{8}{|c|}{ Gestational age (weeks) } \\
\hline & & \multicolumn{2}{|c|}{ 23-27 } & \multicolumn{2}{|c|}{$28-31$} & \multicolumn{2}{|c|}{$32-36$} & \multicolumn{2}{|c|}{ TOTAL } \\
\hline & & No. & $\%$ & No. & $\%$ & No. & $\%$ & No. & $\%$ \\
\hline \multirow[t]{3}{*}{1996} & No & 23 & 11.1 & 64 & 13.8 & 291 & 55.4 & 378 & 31.6 \\
\hline & Yes & 185 & 88.9 & 401 & 86.2 & 234 & 44.6 & 820 & 68.4 \\
\hline & TOTAL & 208 & 100.0 & 465 & 100.0 & 525 & 100.0 & 1198 & 100.0 \\
\hline \multirow[t]{3}{*}{1997} & No & 20 & 9.7 & 80 & 17.1 & 263 & 50.9 & 363 & 30.5 \\
\hline & Yes & 186 & 90.3 & 387 & 82.9 & 254 & 49.1 & 827 & 69.5 \\
\hline & TOTAL & 206 & 100.0 & 467 & 100.0 & 517 & 100.0 & 1190 & 100.0 \\
\hline \multirow[t]{3}{*}{1998} & No & 37 & 14.7 & 70 & 13.6 & 274 & 52.9 & 381 & 29.7 \\
\hline & Yes & 214 & 85.3 & 444 & 86.4 & 244 & 47.1 & 902 & 70.3 \\
\hline & TOTAL & 251 & 100.0 & 514 & 100.0 & 518 & 100.0 & 1283 & 100.0 \\
\hline \multirow[t]{3}{*}{1999} & No & 27 & 10.6 & 57 & 12.0 & 273 & 47.9 & 357 & 27.5 \\
\hline & Yes & 227 & 89.4 & 419 & 88.0 & 297 & 52.1 & 943 & 72.5 \\
\hline & TOTAL & 254 & 100.0 & 476 & 100.0 & 570 & 100.0 & 1300 & 100.0 \\
\hline \multirow[t]{3}{*}{2000} & No & 45 & 18.5 & 64 & 12.5 & 287 & 50.9 & 396 & 30.0 \\
\hline & Yes & 198 & 81.5 & 448 & 87.5 & 277 & 49.1 & 923 & 70.0 \\
\hline & TOTAL & 243 & 100.0 & 512 & 100.0 & 564 & 100.0 & 1319 & 100.0 \\
\hline
\end{tabular}

Source: NICUS Data Collection. NSW Centre for Perinatal Health Services Research. 


\subsection{TRANSFER STATUS, LABOUR AND DELIVERY}

Infants are admitted to a neonatal intensive care unit after:

- delivery which has been booked to occur in a tertiary centre;

- delivery in a tertiary centre following maternal transfer;

- delivery in a non-tertiary centre followed by infant transfer to a tertiary centre.

Thirty-six per cent of all births were booked at a tertiary centre, ranging from 38.9 per cent for the 32-36 week gestational age group to 33.3 per cent for the 37-plus week gestational age group (Table 62). Maternal transfer was most common at gestations less than 32 weeks. The rate of maternal transfer was similar for infants born before 28 weeks gestation (54.2 per cent) and for those born at 28 31 weeks gestation (56.2 per cent). The overall rate of maternal transfer was 34.1 per cent.

Thirty per cent of infants were transferred to a tertiary centre following birth. There were 4.7 per cent $(94 / 2,003)$ of infants transferred from one tertiary centre to another within four hours of commencing assisted ventilation or for major surgery. Transfer following birth was most common in the 37 -plus weeks gestational age group (64.8 per cent). Thirtyseven infants $(37 / 1,123 ; 3.3$ per cent) greater than 31 weeks gestation were discharged home prior to the admission that qualified them for registration in NICUS.

The inverse relationship between gestational age groups and the proportion of births in a tertiary centre is shown in Figure 7 and Table 63. The proportion of infants born in a tertiary centre increased from 61.3 per cent in 1992 to 74.7 per cent 2000 . In $2000,91.7$ per cent of infants less than 32 weeks gestation were born in a tertiary centre compared with 73.4 per cent of 32-36 week gestation infants and 47.7 per cent of term infants.

The pattern of transfer status (Table 64) and place of birth (Table 65) by birthweight is similar to that of gestational age, with the majority (92.4 per cent) of the very low birthweight infants (less than 1,500 grams) born in a tertiary centre.

Spontaneous onset of labour was more common among mothers of infants less than 28 weeks gestation (Table 66). Augmentation and induction of labour were most common in term and post-term births.

The proportion of mothers who gave birth by elective caesarean section (caesarean section without labour) increased from 27.5 per cent in 1992 to 31.9 per cent in 2000. Spontaneous onset of labour occurred in just over twothirds (68.3 per cent) of all infants less than 2,500 grams birth weight (Table 67). As expected, augmentation or induction of labour was most common in mothers of infants with a birthweight of 2,500 grams or more (31.9 per cent).

Prolonged rupture of membranes (greater than 24 hours) was more common at lower gestations, affecting 28 per cent of infants less than 28 weeks gestation (Table 68).

The most common type of delivery was caesarean section (53.1 per cent in 1992 to 52.1 per cent in 2000), followed by normal vaginal delivery (41.9 per cent in 1993 to 37.6 per cent in 2000) and vaginal breech delivery (7.0 per cent in 1998 to 4.6 per cent in 2000) (Tables 69 and 70). The high rate of caesarean section and breech delivery in the NICUS cohort is related to the high proportion of preterm births. The rate of caesarean section in term and post-term births was 37.9 per cent, almost double the rate for all births in NSW and the ACT in 2000.

continued on p.59

FIGURE 7

NICUS REGISTRANTS BY PLACE OF BIRTH (LEVEL OF OBSTETRIC HOSPITAL) AND GESTATIONAL AGE, NSW \& ACT 2000

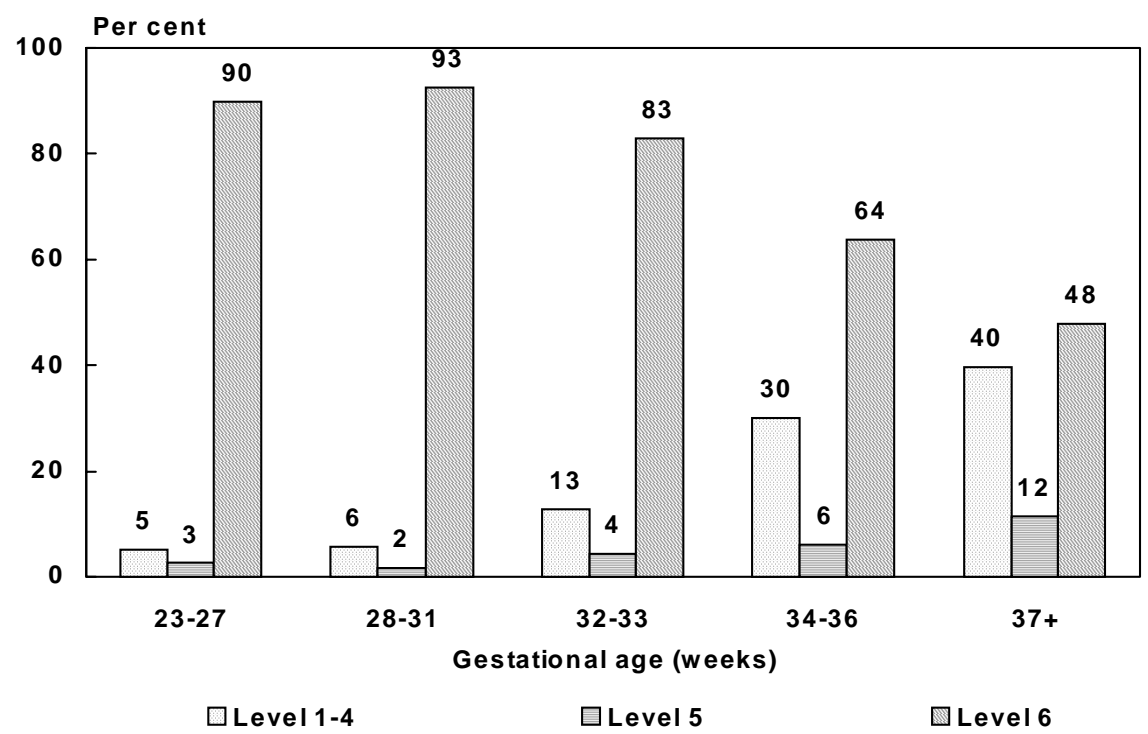

Source: NICUS Data Collection. NSW Centre for Perinatal Health Services Research. 


\section{TABLE 62}

NICUS REGISTRANTS BY BOOKING STATUS, TRANSFER STATUS AND GESTATIONAL AGE, NSW \& ACT 2000

\begin{tabular}{|c|c|c|c|c|c|c|c|c|c|c|c|c|}
\hline \multirow{3}{*}{$\begin{array}{l}\text { Booking status and } \\
\text { transfer status }\end{array}$} & \multicolumn{12}{|c|}{ Gestational age (weeks) } \\
\hline & \multicolumn{2}{|c|}{ 23-27 } & \multicolumn{2}{|c|}{$28-31$} & \multicolumn{2}{|c|}{$32-36$} & \multicolumn{2}{|c|}{$37-41$} & \multicolumn{2}{|c|}{$42+$} & \multicolumn{2}{|c|}{ TOTAL } \\
\hline & No. & $\%$ & No. & $\%$ & No. & $\%$ & No. & $\%$ & No. & $\%$ & No. & $\%$ \\
\hline Booked at tertiary hosp & 98 & 35.6 & 218 & 36.0 & 234 & 38.9 & 170 & 33.2 & 4 & 40.0 & 724 & 36.1 \\
\hline Transfer before birth & 149 & 54.2 & 340 & 56.2 & 185 & 30.8 & 10 & 2.0 & 0 & 0.0 & 684 & 34.1 \\
\hline Transfer after birth & 28 & 10.2 & 47 & 7.8 & 182 & 30.3 & 332 & 6.8 & 6 & 60.0 & 595 & 29.7 \\
\hline TOTAL & 275 & 100.0 & 605 & 100.0 & 601 & 100.0 & 512 & 100.0 & 10 & 100.0 & 2003 & 100.0 \\
\hline
\end{tabular}

Source: NICUS Data Collection. NSW Centre for Perinatal Health Services Research.

\section{TABLE 63}

NICUS REGISTRANTS BY PLACE OF BIRTH (LEVEL OF OBSTETRIC HOSPITAL) AND GESTATIONAL AGE, NSW \& ACT 2000

\begin{tabular}{|c|c|c|c|c|c|c|c|c|c|c|c|c|}
\hline \multirow[t]{3}{*}{ Place of birth } & \multicolumn{12}{|c|}{ Gestational age (weeks) } \\
\hline & \multicolumn{2}{|c|}{$23-27$} & \multicolumn{2}{|c|}{$28-31$} & \multicolumn{2}{|c|}{$32-33$} & \multicolumn{2}{|c|}{$34-36$} & \multicolumn{2}{|c|}{$37+$} & \multicolumn{2}{|c|}{ TOTAL } \\
\hline & No. & $\%$ & No. & $\%$ & No. & $\%$ & No. & $\%$ & No. & $\%$ & No. & $\%$ \\
\hline Level 1-4 & 14 & 5.1 & $34 \#$ & 5.6 & 38 & 12.7 & 91 & 30.2 & 207 & 39.7 & 384 & 19.2 \\
\hline Level 5 & 7 & 2.5 & $10 \#$ & 1.7 & 13 & 4.3 & 18 & 6.0 & 61 & 11.7 & 109 & 5.4 \\
\hline Level 6 & 247 & 89.8 & $560 \#$ & 92.6 & 249 & 83.0 & 192 & 63.8 & 249 & 47.7 & 1497 & 74.7 \\
\hline Born before arrival & 7 & 2.5 & 1 & 0.2 & 0 & 0.0 & 0 & 0.0 & 5 & 1.0 & 13 & 0.6 \\
\hline TOTAL & 275 & 100.0 & 605 & 100.0 & 300 & 100.0 & 301 & 100.0 & 522 & 100.0 & 2003 & 100.0 \\
\hline
\end{tabular}

Source: NICUS Data Collection. NSW Centre for Perinatal Health Services Research.

\# $\quad 23 / 44$ (52.3\%) babies not born in a level six hospital were 30-31 weeks gestation. $324 / 560(57.9 \%)$ babies born in a level six hospital were 30-31 weeks gestation.

\section{TABLE 64}

NICUS REGISTRANTS BY BOOKING STATUS, TRANSFER STATUS AND BIRTHWEIGHT, NSW \& ACT 2000

\begin{tabular}{|c|c|c|c|c|c|c|c|c|c|c|}
\hline \multirow[t]{2}{*}{$\begin{array}{l}\text { Booking status and } \\
\text { transfer status }\end{array}$} & \multicolumn{2}{|c|}{ Less than 1,000} & \multicolumn{4}{|c|}{$\begin{array}{c}\text { Birthweight (grams) } \\
1,500-2,499\end{array}$} & \multicolumn{2}{|c|}{$2,500+$} & \multicolumn{2}{|c|}{ TOTAL } \\
\hline & No. & $\%$ & No. & $\%$ & No. & $\%$ & No. & $\%$ & No. & $\%$ \\
\hline Booked at tertiary hosp & 111 & 39.2 & 172 & 35.0 & 227 & 37.8 & 214 & 34.0 & 724 & 36.1 \\
\hline Transfer before birth & 155 & 54.8 & 277 & 56.4 & 224 & 37.3 & 28 & 4.5 & 684 & 34.1 \\
\hline Transfer after birth & 17 & 6.0 & 42 & 8.6 & 149 & 24.8 & 387 & 61.5 & 595 & 29.7 \\
\hline TOTAL & 283 & 100.0 & 491 & 100.0 & 600 & 100.0 & 629 & 100.0 & 2003 & 100.0 \\
\hline
\end{tabular}

Source: NICUS Data Collection. NSW Centre for Perinatal Health Services Research.

\section{TABLE 65}

NICUS REGISTRANTS BY PLACE OF BIRTH (LEVEL OF OBSTETRIC HOSPITAL) AND BIRTHWEIGHT, NSW \& ACT 2000

\begin{tabular}{|c|c|c|c|c|c|c|c|c|c|c|}
\hline \multirow[t]{2}{*}{ Place of birth } & \multicolumn{10}{|c|}{ Birthweight (grams) } \\
\hline & No. & $\%$ & No. & $\%$ & No. & $\%$ & No. & $\%$ & No. & $\%$ \\
\hline Level 1-4 & 10 & 3.5 & 29 & 5.9 & 97 & 16.2 & 248 & 39.4 & 384 & 19.2 \\
\hline Level 5 & 2 & 0.7 & 9 & 1.8 & 32 & 5.3 & 66 & 10.5 & 109 & 5.4 \\
\hline Level 6 & 266 & 94.0 & 451 & 91.9 & 470 & 78.3 & 310 & 49.3 & 1497 & 74.7 \\
\hline Born before arrival & 5 & 1.8 & 2 & 0.4 & 1 & 0.2 & 5 & 0.8 & 13 & 0.6 \\
\hline TOTAL & 283 & 100.0 & 491 & 100.0 & 600 & 100.0 & 629 & 100.0 & 2003 & 100.0 \\
\hline
\end{tabular}

Source: NICUS Data Collection. NSW Centre for Perinatal Health Services Research. 


\section{TABLE 66}

CONFINEMENTS BY ONSET OF LABOUR AND GESTATIONAL AGE, NSW \& ACT 2000

Onset of labour Gestational age (weeks)

\begin{tabular}{|c|c|c|c|c|c|c|c|c|c|c|c|c|}
\hline \multirow[t]{3}{*}{ Onset of labour } & \multicolumn{12}{|c|}{ Gestational age (weeks) } \\
\hline & \multicolumn{2}{|c|}{ 23-27 } & \multicolumn{2}{|c|}{$28-31$} & \multicolumn{2}{|c|}{$32-36$} & \multicolumn{2}{|c|}{$37-41$} & \multicolumn{2}{|c|}{ 42+ } & \multicolumn{2}{|c|}{ TOTAL } \\
\hline & No. & $\%$ & No. & $\%$ & No. & $\%$ & No. & $\%$ & No. & $\%$ & No. & $\%$ \\
\hline Spontaneous & 169 & 69.5 & 274 & 53.5 & 271 & 48.0 & 237 & 46.3 & 4 & 40.0 & 955 & 51.9 \\
\hline Augmented & 2 & 0.8 & 23 & 4.5 & 24 & 4.3 & 60 & 11.7 & 0 & 0.0 & 109 & 5.9 \\
\hline Induced & 5 & 2.1 & 8 & 1.6 & 44 & 7.8 & 127 & 24.8 & 5 & 50.0 & 189 & 10.3 \\
\hline No labour & 67 & 27.6 & 207 & 40.4 & 225 & 39.9 & 88 & 17.2 & 1 & 10.0 & 588 & 31.9 \\
\hline TOTAL & 243 & 100.0 & 512 & 100.0 & 564 & 100.0 & 512 & 100.0 & 10 & 100.0 & 1841 & 100.0 \\
\hline
\end{tabular}

Source: NICUS Data Collection. NSW Centre for Perinatal Health Services Research.

\section{TABLE 67}

CONFINEMENTS BY ONSET OF LABOUR AND BIRTHWEIGHT, NSW \& ACT 2000

\begin{tabular}{|c|c|c|c|c|c|c|c|c|c|c|}
\hline \multirow[t]{3}{*}{ Onset of labour } & \multicolumn{10}{|c|}{ Birthweight (grams) } \\
\hline & \multicolumn{2}{|c|}{ Less than 1,000} & \multicolumn{2}{|c|}{$1,000-1,499$} & \multicolumn{2}{|c|}{$1,500-2,499$} & \multicolumn{2}{|c|}{$2,500+$} & \multicolumn{2}{|c|}{ TOTAL } \\
\hline & No. & $\%$ & No. & $\%$ & No. & $\%$ & No. & $\%$ & No. & $\%$ \\
\hline Spontaneous & 137 & 55.2 & 209 & 49.1 & 306 & 56.8 & 303 & 48.2 & 955 & 51.9 \\
\hline Augmented & 2 & 0.8 & 15 & $\begin{array}{r}4 J .1 \\
3.5\end{array}$ & 30 & 5.6 & 62 & $\begin{array}{r}40.2 \\
9.9\end{array}$ & $\begin{array}{l}950 \\
109\end{array}$ & $\begin{array}{r}51.9 \\
5.9\end{array}$ \\
\hline Induced & 5 & 2.0 & 8 & 1.9 & 38 & 7.1 & 138 & 22.0 & 189 & 10.3 \\
\hline No labour & 104 & 41.9 & 194 & 45.5 & 165 & 30.6 & 125 & 19.9 & 588 & 31.9 \\
\hline TOTAL & 248 & 100.0 & 426 & 100.0 & 539 & 100.0 & 628 & 100.0 & 1841 & 100.0 \\
\hline
\end{tabular}

Source: NICUS Data Collection. NSW Centre for Perinatal Health Services Research.

\section{TABLE 68}

NICUS REGISTRANTS BY DURATION OF RUPTURE OF MEMBRANES AND GESTATIONAL AGE, NSW \& ACT 2000

\begin{tabular}{|c|c|c|c|c|c|c|c|c|c|c|c|c|}
\hline \multirow{3}{*}{$\begin{array}{l}\text { Duration of rupture of } \\
\text { membranes }\end{array}$} & \multicolumn{12}{|c|}{ Gestational age (weeks) } \\
\hline & \multicolumn{2}{|c|}{ 23-27 } & \multicolumn{2}{|c|}{ 28-31 } & \multicolumn{2}{|c|}{$32-36$} & \multicolumn{2}{|c|}{$37-41$} & \multicolumn{2}{|c|}{$42+$} & \multicolumn{2}{|c|}{ TOTAL } \\
\hline & No. & $\%$ & No. & $\%$ & No. & $\%$ & No. & $\%$ & No. & $\%$ & No. & $\%$ \\
\hline $\begin{array}{l}\text { Less than } 24 \text { hours } \\
24 \text { hours }-7 \text { days } \\
\text { 8+ days } \\
\text { TOTAL }\end{array}$ & $\begin{array}{r}198 \\
38 \\
39 \\
275\end{array}$ & $\begin{array}{r}72.0 \\
13.8 \\
14.2 \\
100.0\end{array}$ & $\begin{array}{r}459 \\
89 \\
57 \\
605\end{array}$ & $\begin{array}{r}75.9 \\
14.7 \\
9.4 \\
100.0\end{array}$ & $\begin{array}{r}514 \\
62 \\
25 \\
601\end{array}$ & $\begin{array}{r}85.5 \\
10.3 \\
4.2 \\
100.0\end{array}$ & $\begin{array}{r}486 \\
22 \\
4 \\
512\end{array}$ & $\begin{array}{r}94.9 \\
4.3 \\
0.8 \\
100.0\end{array}$ & $\begin{array}{r}10 \\
0 \\
0 \\
10\end{array}$ & $\begin{array}{r}100.0 \\
0.0 \\
0.0 \\
100.0\end{array}$ & $\begin{array}{r}1667 \\
211 \\
125 \\
2003\end{array}$ & $\begin{array}{r}83.2 \\
10.5 \\
6.2 \\
100.0\end{array}$ \\
\hline
\end{tabular}

Source: NICUS Data Collection. NSW Centre for Perinatal Health Services Research.

\section{TABLE 69}

NICUS REGISTRANTS BY TYPE OF DELIVERY AND GESTATIONAL AGE, NSW \& ACT 2000

\begin{tabular}{|c|c|c|c|c|c|c|c|c|c|c|c|c|}
\hline \multirow[t]{3}{*}{ Type of delivery } & \multicolumn{12}{|c|}{ Gestational age (weeks) } \\
\hline & \multicolumn{2}{|c|}{ 23-27 } & \multicolumn{2}{|c|}{ 28-31 } & \multicolumn{2}{|c|}{$32-36$} & \multicolumn{2}{|c|}{$37-41$} & \multicolumn{2}{|c|}{$42+$} & \multicolumn{2}{|c|}{ TOTAL } \\
\hline & No. & $\%$ & No. & $\%$ & No. & $\%$ & No. & $\%$ & No. & $\%$ & No. & $\%$ \\
\hline Normal vaginal & 102 & 37.1 & 193 & 31.9 & 207 & 34.4 & 246 & 48.0 & 6 & 60.0 & 754 & 37.6 \\
\hline Forceps & 5 & 1.8 & 7 & 1.2 & 14 & 2.3 & 19 & 3.7 & 0 & 0.0 & 45 & 2.2 \\
\hline Forceps rotation & 0 & 0.0 & 1 & 0.2 & 7 & 1.2 & 8 & 1.6 & 0 & 0.0 & 16 & 0.8 \\
\hline Vacuum extraction & 0 & 0.0 & 2 & 0.3 & 13 & 2.2 & 36 & 7.0 & 0 & 0.0 & 51 & 2.5 \\
\hline Vaginal breech & 29 & 10.5 & 40 & 6.6 & 15 & 2.5 & 9 & 1.8 & 0 & 0.0 & 93 & 4.6 \\
\hline Elective Caesarean & 75 & 27.3 & 239 & 39.5 & 247 & 41.1 & 94 & 18.4 & 1 & 10.0 & 656 & 32.8 \\
\hline Emergency Caesarean & 64 & 23.3 & 123 & 20.3 & 98 & 16.3 & 100 & 19.5 & 3 & 30.0 & 388 & 19.4 \\
\hline TOTAL & 275 & 100.0 & 605 & 100.0 & 601 & 100.0 & 512 & 100.0 & 10 & 100.0 & 2003 & 100.0 \\
\hline
\end{tabular}

Source: NICUS Data Collection. NSW Centre for Perinatal Health Services Research. 


\section{TABLE 70}

\section{NICUS REGISTRANTS BY TYPE OF DELIVERY AND BIRTHWEIGHT, NSW \& ACT 2000}

\begin{tabular}{|c|c|c|c|c|c|c|c|c|c|c|}
\hline \multirow[t]{2}{*}{ Type of delivery } & \multicolumn{10}{|c|}{ Birthweight (grams) } \\
\hline & No. & $\%$ & No. & $\%$ & No. & $\%$ & No. & $\%$ & No. & $\%$ \\
\hline Normal vaginal & 81 & 28.6 & 135 & 27.5 & 251 & 41.8 & 287 & 45.6 & 754 & 37.6 \\
\hline Forceps & 3 & 1.1 & 7 & 1.4 & 16 & 2.7 & 19 & 3.0 & 45 & 2.2 \\
\hline Forceps rotation & 0 & 0.0 & 2 & 0.4 & 5 & 0.8 & 9 & 1.4 & 16 & 0.8 \\
\hline Vacuum extraction & 0 & 0.0 & 0 & 0.0 & 9 & 1.5 & 42 & 6.7 & 51 & 2.5 \\
\hline Vaginal breech & 24 & 8.5 & 32 & 6.5 & 25 & 4.2 & 12 & 1.9 & 93 & 4.6 \\
\hline Elective Caesarean & 119 & 42.0 & 223 & 45.4 & 183 & 30.5 & 131 & 20.8 & 656 & 32.8 \\
\hline Emergency Caesarean & 56 & 19.8 & 92 & 18.7 & 111 & 18.5 & 129 & 20.5 & 388 & 19.4 \\
\hline TOTAL & 283 & 100.0 & 491 & 100.0 & 600 & 100.0 & 629 & 100.0 & 2003 & 100.0 \\
\hline
\end{tabular}

Source: NICUS Data Collection. NSW Centre for Perinatal Health Services Research.

\subsection{INFANT CHARACTERISTICS}

Nearly three quarters of the infants (73.9 per cent) were preterm (less than 37 weeks gestation), 43.9 per cent were very preterm (less than 32 weeks gestation) and 13.7 per cent were extremely preterm (less than 28 weeks gestation) (Figure 8). The proportion of infants in each gestational age group has remained constant (Table 71). Almost all live born infants at 24-31 weeks gestation were admitted to a NICU, about two-thirds at 31-32 weeks gestation, and one-fifth at 33-34 weeks gestation (Table 72).

Sixty-nine per cent of infants had a low birthweight (less than 2,500 grams), 38.6 per cent had a very low birthweight (less than 1,500 grams) and 14.1 per cent had an extremely low birthweight (less than 1,000 grams). The proportion of infants in each birthweight group has remained constant (Table 73). Almost all live born infants 600-1500 grams birthweight were admitted to a NICU (Table 74).

Overall, 58.6 per cent of infants were male. The ratio of males to females was approximately $3: 2$ in most gestational age groups (Table 75).

The overall proportion of the infants who had a major congenital anomaly decreased from 20.9 per cent in 1992 to 16.7 per cent in 2000. Congenital anomalies were more common among term infants (37 plus weeks gestational age), of whom 40.4 per cent had a major congenital anomaly and 2.9 per cent had a minor congenital anomaly (Table 76).
The overall proportion of infants born following a multiple pregnancy has remained constant since 1992. In 2000 most of the infants (79.1 per cent) were from a singleton pregnancy, 18.8 per cent were from a twin pregnancy and 2.0 per cent were from a triplet pregnancy. Infants born as a result of a multiple gestation were more likely to be preterm, with 27.1 per cent of infants less than 37 weeks gestation being from a multiple gestation pregnancy (Table 77). Multiple births represented 3.3 per cent of all NSW and ACT births in 2000. The higher than expected rate of multiple births among the 2000 NICUS cohort reflects the high proportion of multiple pregnancies resulting in preterm birth.

Table 78 shows the median, 25th and 75 th percentiles for one- and five-minute Apgar scores according to gestational age groups. For infants greater than 27 weeks gestational age the median one-minute Apgar score was seven and the median five-minute score was nine. The proportion of infants with a one-minute Apgar score of 0-4 has decreased from 38.7 per cent in 1992 to 25.4 per cent in 2000, similarly the proportion of infants with a five-minute Apgar score of 0-4 has decreased from 10.8 per cent in 1992 to 7.7 per cent in 2000 (Table 79).

continued on p.63

\section{TABLE 71}

NICUS REGISTRANTS BY GESTATIONAL AGE, NSW \& ACT 1996-2000

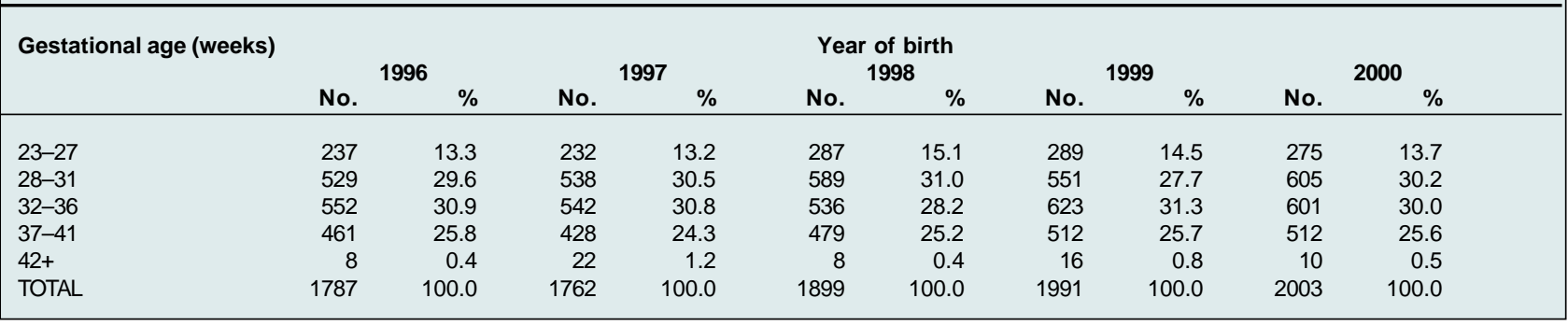

Source: NICUS Data Collection. NSW Centre for Perinatal Health Services Research. 


\section{FIGURE 8}

NICUS REGISTRANTS BY GESTATIONAL AGE, NSW \& ACT 2000

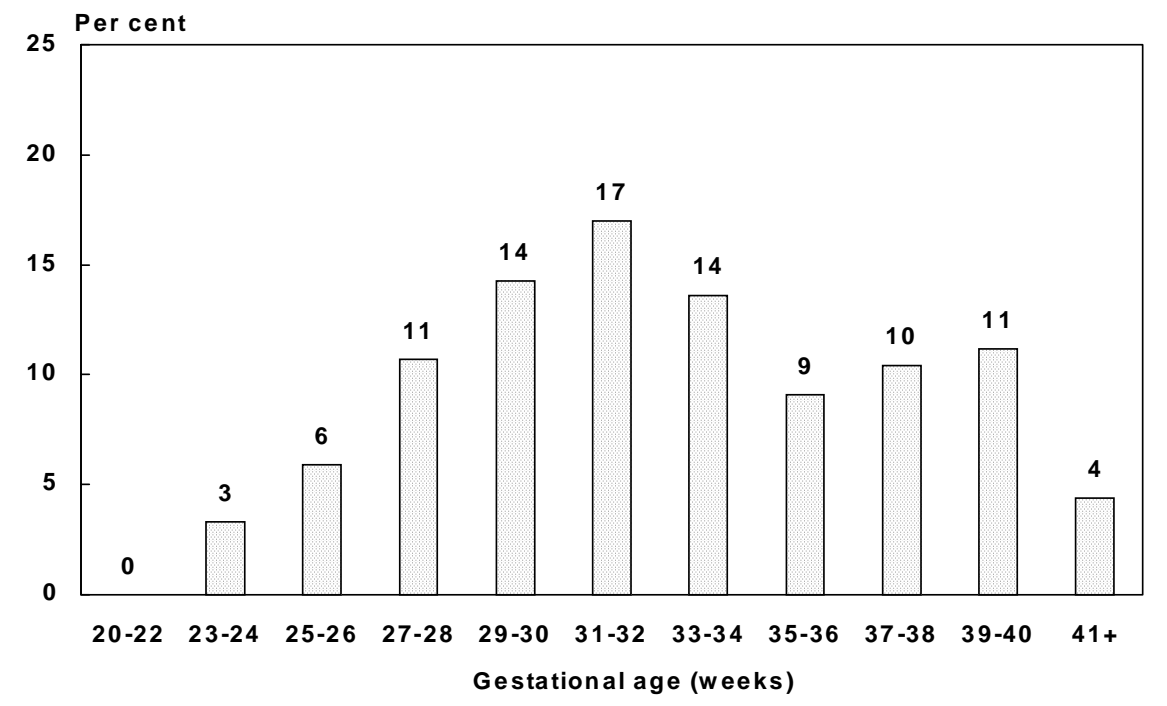

Source: NICUS Data Collection. NSW Centre for Perinatal Health Services Research.

\section{TABLE 72}

BIRTHS BY NICUS REGISTRATION AND GESTATIONAL AGE, NSW \& ACT 2000*

\begin{tabular}{|c|c|c|c|c|c|}
\hline \multirow{2}{*}{$\begin{array}{l}\text { Gestational age } \\
\text { (weeks) }\end{array}$} & \multicolumn{2}{|c|}{ NSW \& ACT } & \multicolumn{3}{|c|}{ NICUS } \\
\hline & $\begin{array}{c}\text { Stillbirths } \\
\text { No. }\end{array}$ & $\begin{array}{l}\text { Live births } \\
\text { No. }\end{array}$ & $\begin{array}{c}\text { Registrations } \\
\text { No. }\end{array}$ & $\begin{array}{l}\text { Rate per } 1,000 \\
\text { live births }\end{array}$ & $\begin{array}{c}\% \\
\text { of cohort }\end{array}$ \\
\hline Less than 21 & 50 & 11 & 0 & 0.0 & 0.0 \\
\hline 21 & 64 & 21 & 0 & 0.0 & 0.0 \\
\hline 22 & 49 & 38 & 0 & 0.0 & 0.0 \\
\hline 23 & 36 & 25 & 17 & 680.0 & 0.8 \\
\hline 24 & 30 & 49 & 49 & 1000.0 & 2.4 \\
\hline 25 & 28 & 64 & 40 & 625.0 & 2.0 \\
\hline 26 & 24 & 87 & 78 & 896.6 & 3.9 \\
\hline 27 & 15 & 87 & 91 & 1046.0 & 4.5 \\
\hline 28 & 10 & 120 & 124 & 1033.3 & 6.2 \\
\hline 29 & 22 & 140 & 134 & 957.1 & 6.7 \\
\hline 30 & 20 & 171 & 153 & 894.7 & 7.6 \\
\hline 31 & 25 & 206 & 194 & 941.7 & 9.7 \\
\hline 32 & 18 & 330 & 147 & 445.5 & 7.3 \\
\hline 33 & 17 & 492 & 153 & 311.0 & 7.6 \\
\hline 34 & 23 & 741 & 119 & 160.6 & 5.9 \\
\hline 35 & 16 & 1264 & 97 & 76.7 & 4.8 \\
\hline 36 & 29 & 2461 & 85 & 34.5 & 4.2 \\
\hline 37 & 28 & 5039 & 81 & 16.1 & 4.0 \\
\hline 38 & 35 & 13718 & 128 & 9.3 & 6.4 \\
\hline 39 & 33 & 20572 & 107 & 5.2 & 5.3 \\
\hline 40 & 38 & 29397 & 117 & 4.0 & 5.8 \\
\hline 41 & 20 & 14624 & 79 & 5.4 & 3.9 \\
\hline 42 & 1 & 2102 & 10 & 4.8 & 0.5 \\
\hline 43 & 1 & 149 & 0 & 0.0 & 0.0 \\
\hline 44 & 1 & 6 & 0 & 0.0 & 0.0 \\
\hline 45 & 0 & 0 & 0 & 0.0 & 0.0 \\
\hline Not stated & 0 & 59 & 0 & 0.0 & 0.0 \\
\hline TOTAL & 633 & 91973 & 2003 & 21.8 & 100.0 \\
\hline
\end{tabular}

Source: NICUS Data Collection. NSW Centre for Perinatal Health Services Research, 2000. NSW Midwives Data Collection. Epidemiology and Surveillance Branch, NSW Department of Health, 2000. ACT Maternal-Perinatal Data Collection, 1999.

\# Excludes four babies reported to the MDC in 2000 for whom the birth outcome was not known. 


\section{TABLE 73}

NICUS REGISTRANTS BY BIRTHWEIGHT, NSW \& ACT 2000

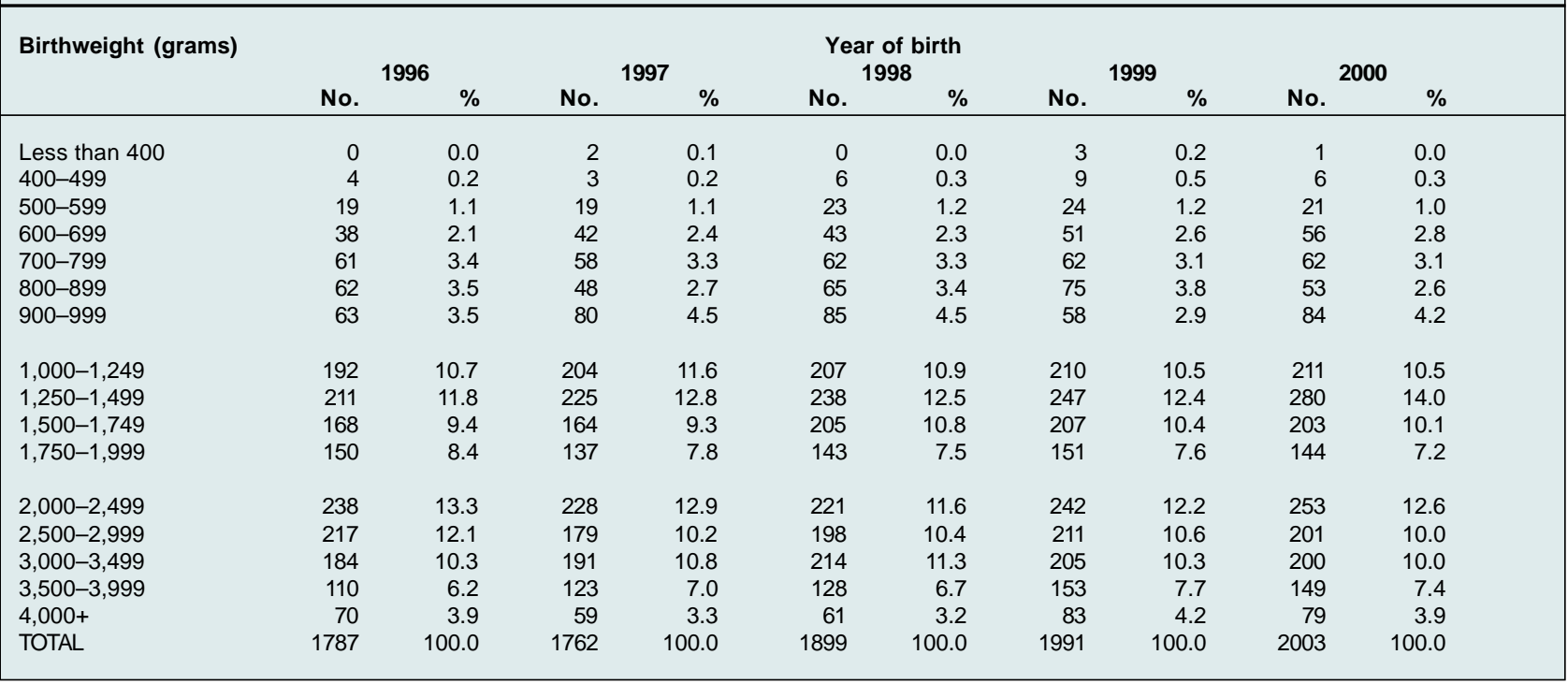

Source: NICUS Data Collection. NSW Centre for Perinatal Health Services Research.

\section{TABLE 74}

NICUS REGISTRANTS BY NICUS REGISTRATION AND BIRTHWEIGHT, NSW \& ACT 2000\#

\begin{tabular}{|c|c|c|c|c|c|}
\hline \multirow{2}{*}{$\begin{array}{l}\text { Birthweight } \\
\text { (grams) }\end{array}$} & \multicolumn{2}{|c|}{ NSW \& ACT } & \multirow[b]{2}{*}{$\begin{array}{c}\text { Registrations } \\
\text { No. } \\
\end{array}$} & \multirow{2}{*}{$\begin{array}{c}\text { NICUS } \\
\text { Rate per } 1,000 \\
\text { live births }\end{array}$} & \multirow[b]{2}{*}{$\begin{array}{c}\% \\
\text { of cohort }\end{array}$} \\
\hline & $\begin{array}{c}\text { Stillbirths } \\
\text { No. }\end{array}$ & $\begin{array}{c}\text { Live births }{ }^{\#} \\
\text { No. }\end{array}$ & & & \\
\hline Less than 400 & 124 & 54 & 1 & 18.5 & 0.0 \\
\hline $400-499$ & 73 & 40 & 6 & 150.0 & 0.3 \\
\hline 500-599 & 40 & 48 & 21 & 437.5 & 1.0 \\
\hline $600-699$ & 32 & 70 & 56 & 800.0 & 2.8 \\
\hline $700-799$ & 25 & 72 & 62 & 861.1 & 3.1 \\
\hline $800-899$ & 20 & 56 & 53 & 946.4 & 2.6 \\
\hline $900-999$ & 12 & 89 & 84 & 943.8 & 4.2 \\
\hline $1,000-1,249$ & 28 & 233 & 211 & 905.6 & 10.5 \\
\hline $1,250-1,499$ & 25 & 303 & 280 & 924.1 & 14.0 \\
\hline $1,500-1,749$ & 19 & 437 & 203 & 464.5 & 10.1 \\
\hline $1,750-1,999$ & 16 & 662 & 144 & 217.5 & 7.2 \\
\hline $2,000-2,499$ & 56 & 3503 & 253 & 72.2 & 12.6 \\
\hline $2,500-2,999$ & 65 & 13402 & 201 & 15.0 & 10.0 \\
\hline $3,000-3,499$ & 42 & 32197 & 200 & 6.2 & 10.0 \\
\hline 3,500-3,999 & 38 & 28903 & 149 & 5.2 & 7.4 \\
\hline $4,000+$ & 17 & 11899 & 79 & 6.6 & 3.9 \\
\hline Not stated & 1 & 5 & 0 & 0.0 & 0.0 \\
\hline TOTAL & 633 & 91973 & 2003 & 21.8 & 100.0 \\
\hline
\end{tabular}

Source: NICUS Data Collection. NSW Centre for Perinatal Health Services Research, 2000. NSW Midwives Data Collection. Epidemiology and Surveillance Branch, NSW Health Department, 2000. ACT Maternal-Perinatal Data Collection, 1999.

Excludes 4 babies reported to the MDC in 2000 for whom the birth outcome was not known.

\section{TABLE 75}

NICUS REGISTRANTS BY GENDER AND GESTATIONAL AGE, NSW \& ACT 2000

\begin{tabular}{|c|c|c|c|c|c|c|c|c|c|c|c|c|}
\hline \multirow{3}{*}{ Sex } & \multicolumn{12}{|c|}{ Gestational age (weeks) } \\
\hline & \multicolumn{2}{|c|}{ 23-27 } & \multicolumn{2}{|c|}{ 28-31 } & \multicolumn{2}{|c|}{$32-36$} & \multicolumn{2}{|c|}{$37-41$} & \multicolumn{2}{|c|}{ 42+ } & \multicolumn{2}{|c|}{ TOTAL } \\
\hline & No. & $\%$ & No. & $\%$ & No. & $\%$ & No. & $\%$ & No. & $\%$ & No. & $\%$ \\
\hline Male & 152 & 55.3 & 331 & 54.7 & 369 & 61.4 & 316 & 61.7 & 6 & 60.0 & 1174 & 58.6 \\
\hline Female & 123 & 44.7 & 274 & 45.3 & 232 & 38.6 & 196 & 38.3 & 4 & 40.0 & 829 & 41.4 \\
\hline TOTAL & 275 & 100.0 & 605 & 100.0 & 601 & 100.0 & 512 & 100.0 & 10 & 100.0 & 2003 & 100.0 \\
\hline
\end{tabular}

Source: NICUS Data Collection. NSW Centre for Perinatal Health Services Research. 
TABLE 76

NICUS REGISTRANTS BY CONGENITAL ANOMALIES AND GESTATIONAL AGE, NSW \& ACT 2000

\begin{tabular}{|c|c|c|c|c|c|c|c|c|c|c|c|c|}
\hline \multirow[t]{3}{*}{ Congenital anomaly } & \multicolumn{12}{|c|}{ Gestational age (weeks) } \\
\hline & \multicolumn{2}{|c|}{$23-27$} & \multicolumn{2}{|c|}{$28-31$} & \multicolumn{2}{|c|}{$32-36$} & \multicolumn{2}{|c|}{$37-41$} & \multicolumn{2}{|c|}{$42+$} & \multicolumn{2}{|c|}{ TOTAL } \\
\hline & No. & $\%$ & No. & $\%$ & No. & $\%$ & No. & $\%$ & No. & $\%$ & No. & $\%$ \\
\hline None & 254 & 92.4 & 556 & 91.9 & 510 & 84.9 & 285 & 55.7 & 6 & 60.0 & 1611 & 80.4 \\
\hline Minor & 8 & 2.9 & 14 & 2.3 & 16 & 2.7 & 19 & 3.7 & 1 & 10.0 & 58 & 2.9 \\
\hline Major & 13 & 4.7 & 35 & 5.8 & 75 & 12.5 & 208 & 40.6 & 3 & 30.0 & 334 & 16.7 \\
\hline TOTAL & 275 & 100.0 & 605 & 100.0 & 601 & 100.0 & 512 & 100.0 & 10 & 100.0 & 2003 & 100.0 \\
\hline
\end{tabular}

Source: NICUS Data Collection. NSW Centre for Perinatal Health Services Research.

\section{TABLE 77}

NICUS REGISTRANTS BY PLURALITY AND GESTATIONAL AGE, NSW \& ACT 2000

Plurality Gestational age (weeks)

\begin{tabular}{|c|c|c|c|c|c|c|c|c|c|c|c|c|}
\hline \multirow[t]{3}{*}{ 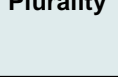 } & \multirow{2}{*}{\multicolumn{2}{|c|}{ 23-27 }} & \multirow{2}{*}{\multicolumn{2}{|c|}{ 28-31 }} & \multirow{2}{*}{\multicolumn{4}{|c|}{$\begin{array}{l}\text { ge (wee } \\
37-41\end{array}$}} & \multirow{2}{*}{\multicolumn{2}{|c|}{$42+$}} & \multirow{2}{*}{\multicolumn{2}{|c|}{ TOTAL }} \\
\hline & & & & & & & & & & & & \\
\hline & No. & $\%$ & No. & $\%$ & No. & $\%$ & No. & $\%$ & No. & $\%$ & No. & $\%$ \\
\hline Singleton & 205 & 74.5 & 417 & 68.9 & 457 & 76.0 & 496 & 96.9 & 10 & 100.0 & 1585 & 79.1 \\
\hline Twins & 60 & 21.8 & 168 & 27.8 & 133 & 22.1 & 16 & 3.1 & 0 & 0.0 & 377 & 18.8 \\
\hline Triplets & 10 & 3.6 & 20 & 3.3 & 11 & 1.8 & 0 & 0.0 & 0 & 0.0 & 41 & 2.0 \\
\hline TOTAL & 275 & 100.0 & 605 & 100.0 & 601 & 100.0 & 512 & 100.0 & 10 & 100.0 & 2003 & 100.0 \\
\hline
\end{tabular}

Source: NICUS Data Collection. NSW Centre for Perinatal Health Services Research.

\section{TABLE 78}

NICUS REGISTRANTS BY APGAR SCORE AND GESTATIONAL AGE, NSW \& ACT 2000

\begin{tabular}{|c|c|c|c|c|c|c|c|c|}
\hline \multirow[t]{2}{*}{ Apgar Score } & \multicolumn{8}{|c|}{ Gestational age (weeks) } \\
\hline & \multicolumn{2}{|c|}{$\begin{array}{c}23-27 \\
\text { Median }(25 \%, 75 \%)\end{array}$} & \multicolumn{2}{|c|}{$\begin{array}{c}28-31 \\
\text { Median (25\%,75\%) }\end{array}$} & \multicolumn{2}{|c|}{$\begin{array}{c}32-36 \\
\text { Median (25\%,75\%) }\end{array}$} & \multicolumn{2}{|c|}{$\begin{array}{c}37+ \\
\text { Median }(25 \%, 75 \%)\end{array}$} \\
\hline One-minute Apgar & 5 & $(3,7)$ & 7 & $(5,8)$ & 7 & $(5,8)$ & 7 & $(4,8)$ \\
\hline Five-minute Apgar & 8 & $(6,8)$ & 9 & $(8,9)$ & 9 & $(8,9)$ & 9 & $(7,9)$ \\
\hline
\end{tabular}

Source: NICUS Data Collection. NSW Centre for Perinatal Health Services Research.

\section{TABLE 79}

NICUS REGISTRANTS BY APGAR SCORE AT ONE AND FIVE MINUTES, NSW \& ACT 1996-2000

\begin{tabular}{|c|c|c|c|c|c|c|c|c|c|c|}
\hline \multirow[t]{3}{*}{ Apgar Score } & \multicolumn{10}{|c|}{ Year of birth } \\
\hline & \multicolumn{2}{|c|}{1996} & \multicolumn{2}{|c|}{1997} & \multicolumn{2}{|c|}{1998} & \multicolumn{2}{|c|}{1999} & \multicolumn{2}{|c|}{2000} \\
\hline & No. & $\%$ & No. & $\%$ & No. & $\%$ & No. & $\%$ & No. & $\%$ \\
\hline \multicolumn{11}{|l|}{ One minute } \\
\hline $0-4$ & 533 & 29.8 & 453 & 25.7 & 533 & 28.1 & 530 & 26.6 & 509 & 25.4 \\
\hline $5-7$ & 655 & 36.7 & 649 & 36.8 & 693 & 36.5 & 689 & 34.6 & 743 & 37.1 \\
\hline $8+$ & 586 & 32.8 & 650 & 36.9 & 657 & 34.6 & 766 & 38.5 & 737 & 36.8 \\
\hline Not stated & 13 & 0.7 & 10 & 0.6 & 16 & 0.8 & 6 & 0.3 & 14 & 0.7 \\
\hline TOTAL & 1787 & 100.0 & 1762 & 100.0 & 1899 & 100.0 & 1991 & 100.0 & 2003 & 100.0 \\
\hline \multicolumn{11}{|l|}{ Five minutes } \\
\hline $0-4$ & 150 & 8.4 & 121 & 6.9 & 142 & 7.5 & 132 & 6.6 & 154 & 7.7 \\
\hline $5-7$ & 453 & 25.3 & 413 & 23.4 & 412 & 21.7 & 436 & 21.9 & 399 & 19.9 \\
\hline $8+$ & 1171 & 65.5 & 1219 & 69.2 & 1329 & 70.0 & 1417 & 71.2 & 1437 & 71.7 \\
\hline Not stated & 13 & 0.7 & 9 & 0.5 & 16 & 0.8 & 6 & 0.3 & 13 & 0.6 \\
\hline TOTAL & 1787 & 100.0 & 1762 & 100.0 & 1899 & 100.0 & 1991 & 100.0 & 2003 & 100.0 \\
\hline
\end{tabular}

Source: NICUS Data Collection. NSW Centre for Perinatal Health Services Research 


\section{continued from p.59}

Infants with major congenital anomalies $(n=334)$ have been excluded from the analysis of morbidity and mortality.

The majority of infants without a major congenital anomaly ( $n=1,669 ; 88.7$ per cent) in the 2000 NICUS cohort received assisted ventilation (intermittent mandatory ventilation and/or continuous positive airways pressure) (Table 80).

The main indication for ventilation for most infants was respiratory distress syndrome (Figure 9). Main indication for assisted ventilation varied with gestational age. Respiratory distress syndrome, immature lung and transient tachypnoea were more common in the preterm groups, whereas perinatal asphyxia, meconium aspiration, pulmonary hypertension and apnoea were more common in term infants (Table 81).

Proven systemic infection has decreased from 22.0 per cent in 1992 to 14.4 per cent of infants in 2000. Infection was most common among infants less than 28 weeks gestation (46.6 per cent) (Table 82).

The overall proportion of ventilated infants who received surfactant has increased from 33.8 per cent in 1992 to 42.6 per cent in 2000 (Table 83). In 2000, 56.2 per cent of the infants who received surfactant were less than 32 weeks gestational age. The majority ( 66.6 per cent) of ventilated infants with a diagnosis of respiratory distress syndrome received surfactant.
Overall, the incidence of treated patent ductus arteriosus (PDA) has decreased from 18.7 per cent in 1993 to 15.4 per cent in 2000. In 2000, 95.2 per cent of the infants treated for PDA were less than 32 weeks gestational age (Table 84). The majority of infants with a PDA requiring treatment received indomethacin only (14.7 per cent). Surgical treatment of PDA was predominantly performed on infants less than 28 weeks gestation ( 2.3 per cent). Some infants ( 0.5 per cent $)$ were treated with both indomethacin and surgery.

Overall, the incidence of necrotising enterocolitis (NEC) has decreased from 9.6 per cent in 1992 to 2.2 per cent in 2000. The diagnosis of NEC was made radiologically or at surgery in 56.8 per cent of infants and clinically in the remainder. NEC was more common at the lower gestational age groups and 75.7 per cent of the infants with NEC were born at less than 32 weeks gestation (Table 85).

The overall incidence of major surgery has decreased from 7.6 per cent in 1992 to 3.0 per cent in 2000. In 2000, 46.0 per cent of the infants who required major surgery were less than 32 weeks gestation (Table 86). The most common surgical procedures amongst these infants were for patent ductus arteriosus and necrotising enterocolitis.

continued on p.66

\section{TABLE 80}

ASSISTED VENTILATION BY GESTATIONAL AGE, NSW \& ACT 1996-2000\#

\begin{tabular}{|c|c|c|c|c|c|c|c|c|c|c|c|}
\hline \multirow[t]{3}{*}{ Year } & \multirow[t]{3}{*}{ Assisted ventilation } & \multicolumn{10}{|c|}{ Gestational age (weeks) } \\
\hline & & \multicolumn{2}{|c|}{ 23-27 } & \multicolumn{2}{|c|}{$28-31$} & \multicolumn{2}{|c|}{$32-36$} & \multicolumn{2}{|c|}{$37+$} & \multicolumn{2}{|c|}{ TOTAL } \\
\hline & & No. & $\%$ & No. & $\%$ & No. & $\%$ & No. & $\%$ & No. & $\%$ \\
\hline \multirow[t]{3}{*}{1996} & No & 1 & 0.4 & 117 & 23.3 & 48 & 10.2 & 6 & 2.2 & 172 & 11.7 \\
\hline & Yes & 227 & 99.6 & 385 & 76.7 & 424 & 89.8 & 267 & 97.8 & 1303 & 88.3 \\
\hline & TOTAL & 228 & 100.0 & 502 & 100.0 & 472 & 100.0 & 273 & 100.0 & 1475 & 100.0 \\
\hline \multirow[t]{3}{*}{1997} & No & 0 & 0.0 & 104 & 20.1 & 60 & 12.9 & 9 & 3.5 & 173 & 11.8 \\
\hline & Yes & 227 & 100.0 & 413 & 79.9 & 406 & 87.1 & 248 & 96.5 & 1294 & 88.2 \\
\hline & TOTAL & 227 & 100.0 & 517 & 100.0 & 466 & 100.0 & 257 & 100.0 & 1467 & 100.0 \\
\hline \multirow[t]{3}{*}{1998} & No & 2 & 0.7 & 99 & 17.6 & 48 & 10.4 & 7 & 2.6 & 156 & 9.9 \\
\hline & Yes & 278 & 99.3 & 465 & 82.4 & 415 & 89.6 & 265 & 97.4 & 1423 & 90.1 \\
\hline & TOTAL & 280 & 100.0 & 564 & 100.0 & 463 & 100.0 & 272 & 100.0 & 1579 & 100.0 \\
\hline \multirow[t]{3}{*}{1999} & No & 1 & 0.4 & 119 & 22.7 & 60 & 11.5 & 9 & 3.0 & 189 & 11.6 \\
\hline & Yes & 280 & 99.6 & 405 & 77.3 & 464 & 88.5 & 292 & 97.0 & 1441 & 88.4 \\
\hline & TOTAL & 281 & 100.0 & 524 & 100.0 & 524 & 100.0 & 301 & 100.0 & 1630 & 100.0 \\
\hline \multirow[t]{3}{*}{2000} & No & 1 & 0.4 & 116 & 20.4 & 65 & 12.4 & 6 & 1.9 & 188 & 11.3 \\
\hline & Yes & 261 & 99.6 & 454 & 79.6 & 461 & 87.6 & 305 & 98.1 & 1481 & 88.7 \\
\hline & TOTAL & 262 & 100.0 & 570 & 100.0 & 526 & 100.0 & 311 & 100.0 & 1669 & 100.0 \\
\hline
\end{tabular}

Source: NICUS Data Collection. NSW Centre for Perinatal Health Services Research.

\# Babies with major congenital anomalies excluded. 


\section{FIGURE 9}

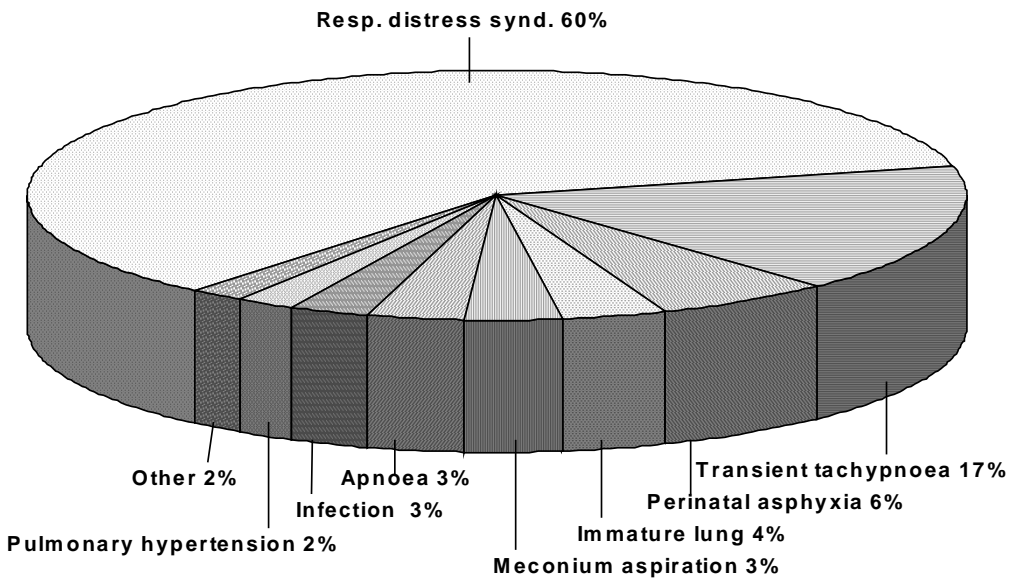

Source: NICUS Data Collection. NSW Centre for Perinatal Health Services Research.

\# Babies with major congenital anomalies or not ventilated excluded.

\section{TABLE 81}

MAIN INDICATION FOR ASSISTED VENTILATION OF BABIES BY GESTATIONAL AGE, NSW \& ACT $2000^{\#}$

\begin{tabular}{|c|c|c|c|c|c|c|c|c|c|c|}
\hline \multirow{3}{*}{ Indication } & \multicolumn{10}{|c|}{ Gestational age (weeks) } \\
\hline & \multicolumn{2}{|c|}{ 23-27 } & \multicolumn{2}{|c|}{ 28-31 } & \multicolumn{2}{|c|}{$32-36$} & \multicolumn{2}{|c|}{$37+$} & \multicolumn{2}{|c|}{ TOTAL } \\
\hline & No. & $\%$ & No. & $\%$ & No. & $\%$ & No. & $\%$ & No. & $\%$ \\
\hline Respiratory distress syndrome & 235 & 90.0 & 313 & 68.9 & 286 & 62.0 & 41 & 13.4 & 875 & 59.0 \\
\hline Transient tachypnoea of newborn & 1 & 0.4 & 69 & 15.2 & 108 & 23.4 & 70 & 23.0 & 248 & 16.7 \\
\hline Meconium aspiration & 0 & 0.0 & 0 & 0.0 & 3 & 0.7 & 45 & 14.8 & 48 & 3.2 \\
\hline Infection & 1 & 0.4 & 9 & 2.0 & 12 & 2.6 & 21 & 6.9 & 43 & 2.9 \\
\hline Perinatal asphyxia & 0 & 0.0 & 5 & 1.1 & 18 & 3.9 & 64 & 21.0 & 87 & 5.9 \\
\hline Immature lung & 22 & 8.4 & 34 & 7.5 & 0 & 0.0 & 0 & 0.0 & 56 & 3.8 \\
\hline Apnoea & 2 & 0.8 & 19 & 4.2 & 14 & 3.0 & 13 & 4.3 & 48 & 3.2 \\
\hline Pulmonary hypertension & 0 & 0.0 & 2 & 0.4 & 3 & 0.7 & 23 & 7.5 & 28 & 1.9 \\
\hline Congenital anomaly & 0 & 0.0 & 0 & 0.0 & 1 & 0.2 & 3 & 1.0 & 4 & 0.3 \\
\hline Cardiac disorder & 0 & 0.0 & 0 & 0.0 & 1 & 0.2 & 3 & 1.0 & 4 & 0.3 \\
\hline Peri surgery & 0 & 0.0 & 2 & 0.4 & 4 & 0.9 & 7 & 2.3 & 13 & 0.9 \\
\hline Other & 0 & 0.0 & 1 & 0.2 & 11 & 2.4 & 15 & 4.9 & 27 & 1.8 \\
\hline TOTAL & 261 & 100.0 & 454 & 100.0 & 461 & 100.0 & 305 & 100.0 & 1481 & 100.0 \\
\hline
\end{tabular}

Source: NICUS Data Collection. NSW Centre for Perinatal Health Services Research.

\# Babies with major congenital anomalies or not ventilated excluded.

\section{TABLE 82}

PROVEN SYSTEMIC INFECTION BY GESTATIONAL AGE, NSW \& ACT 2000"

\begin{tabular}{|c|c|c|c|c|c|c|c|c|c|c|}
\hline \multirow{3}{*}{ Infection } & \multicolumn{10}{|c|}{ Gestational age (weeks) } \\
\hline & \multicolumn{2}{|c|}{$23-27$} & \multicolumn{2}{|c|}{ 28-31 } & \multicolumn{2}{|c|}{$32-36$} & \multicolumn{2}{|c|}{$37+$} & \multicolumn{2}{|c|}{ TOTAL } \\
\hline & No. & $\%$ & No. & $\%$ & No. & $\%$ & No. & $\%$ & No. & $\%$ \\
\hline No & 140 & 53.4 & 492 & 86.3 & 507 & 96.4 & 290 & 93.2 & 1429 & 85.6 \\
\hline Yes & 122 & 46.6 & 78 & 13.7 & 19 & 3.6 & 21 & 6.8 & 240 & 14.4 \\
\hline TOTAL & 262 & 100.0 & 570 & 100.0 & 526 & 100.0 & 311 & 100.0 & 1669 & 100.0 \\
\hline
\end{tabular}

Source: NICUS Data Collection. NSW Centre for Perinatal Health Services Research.

\# Babies with major congenital anomalies excluded. 


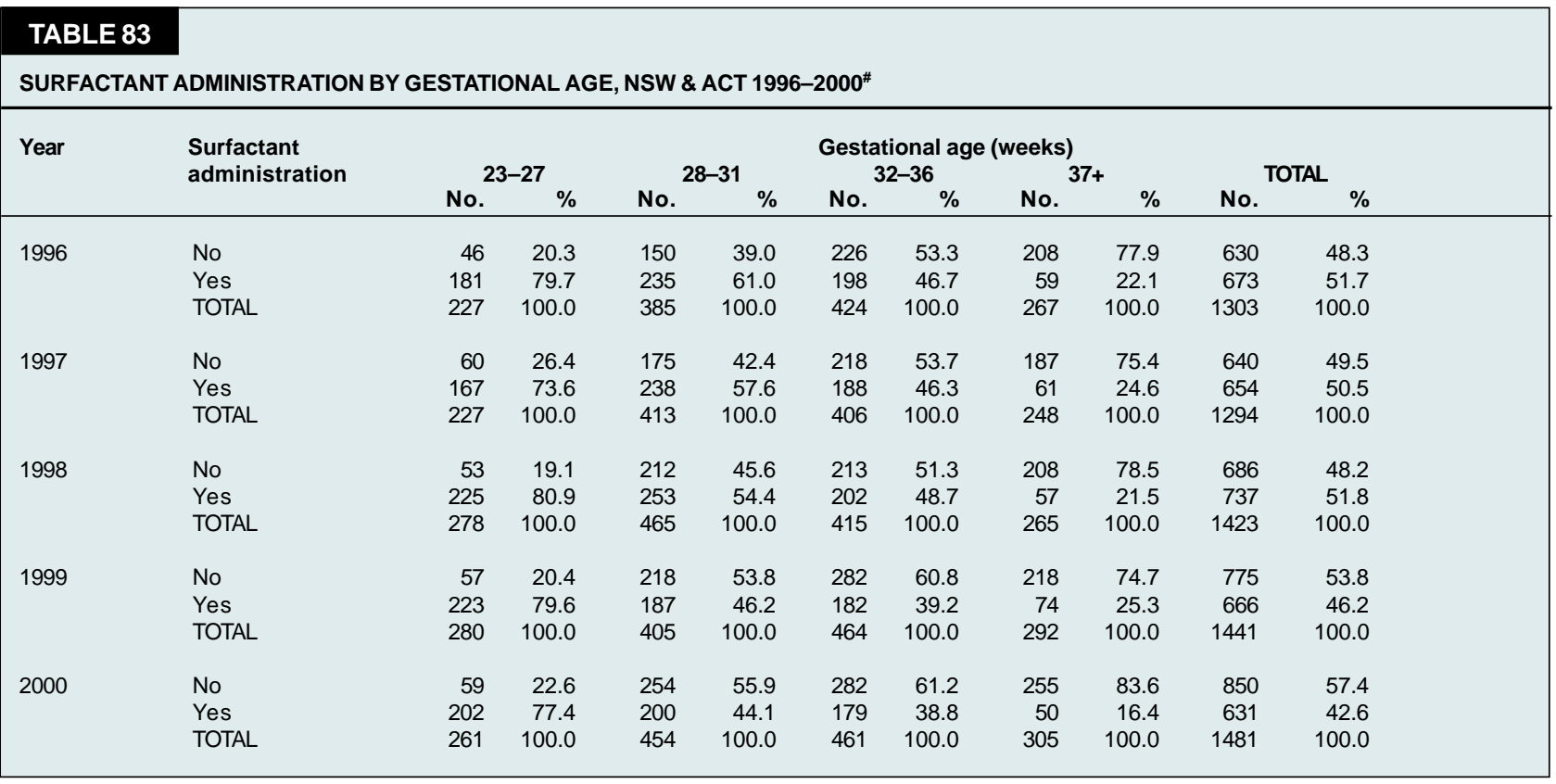

Source: NICUS Data Collection. NSW Centre for Perinatal Health Services Research.

\# Babies with major congenital malformations and babies not ventilated excluded.

\section{TABLE 84}

TREATED PATENT DUCTUS ARTERIOSUS (PDA) BY GESTATIONAL AGE, NSW \& ACT 2000"

\begin{tabular}{|c|c|c|c|c|c|c|c|c|}
\hline \multirow[t]{3}{*}{ PDA-Treatment for PDA } & \multicolumn{8}{|c|}{ Gestational age (weeks) } \\
\hline & \multicolumn{2}{|c|}{ 23-27 } & \multicolumn{2}{|c|}{ 28-31 } & \multicolumn{2}{|c|}{$32-36$} & \multicolumn{2}{|c|}{ TOTAL } \\
\hline & No. & $\%$ & No. & $\%$ & No. & $\%$ & No. & $\%$ \\
\hline No treated PDA & 129 & 49.2 & 503 & 88.2 & 516 & 98.1 & 1148 & 84.5 \\
\hline Indomethacin only & 127 & 48.5 & 64 & 11.2 & 9 & 1.7 & 200 & 14.7 \\
\hline Surgery only & 1 & 0.4 & 1 & 0.2 & 1 & 0.2 & 3 & 0.2 \\
\hline Indomethacin \& surgery & 5 & 1.9 & 2 & 0.4 & 0 & 0.0 & 7 & 0.5 \\
\hline TOTAL & 262 & 100.0 & 570 & 100.0 & 526 & 100.0 & 1358 & 100.0 \\
\hline
\end{tabular}

Source: NICUS Data Collection. NSW Centre for Perinatal Health Services Research.

\# Babies with major congenital anomalies excluded.

\section{TABLE 85}

NECROTISING ENTEROCOLITIS (NEC) BY GESTATIONAL AGE, NSW \& ACT 2000\#

\begin{tabular}{|c|c|c|c|c|c|c|c|c|c|c|}
\hline \multirow[t]{3}{*}{ NEC-Treatment for NEC } & \multicolumn{10}{|c|}{ Gestational age (weeks) } \\
\hline & \multicolumn{2}{|c|}{ 23-27 } & \multicolumn{2}{|c|}{ 28-31 } & \multicolumn{2}{|c|}{$32-36$} & \multicolumn{2}{|c|}{$37+$} & \multicolumn{2}{|c|}{ TOTAL } \\
\hline & No. & $\%$ & No. & $\%$ & No. & $\%$ & No. & $\%$ & No. & $\%$ \\
\hline No NEC & 249 & 95.0 & 555 & 97.4 & 520 & 98.9 & 308 & 99.0 & 1632 & 97.8 \\
\hline Clinical diagnosis & 5 & 1.9 & 7 & 1.2 & 3 & 0.6 & 1 & 0.3 & 16 & 1.0 \\
\hline $\mathrm{X}$-ray diagnosis & 4 & 1.5 & 7 & 1.2 & 1 & 0.2 & 0 & 0.0 & 12 & 0.7 \\
\hline Surgery for NEC & 4 & 1.5 & 1 & 0.2 & 2 & 0.4 & 2 & 0.6 & 9 & 0.5 \\
\hline TOTAL & 262 & 100.0 & 570 & 100.0 & $52 \overline{6}$ & 100.0 & 311 & 100.0 & 1669 & 100.0 \\
\hline
\end{tabular}

Source: NICUS Data Collection. NSW Centre for Perinatal Health Services Research.

\# Babies with major congenital anomalies excluded. 


\section{continued from p.63}

The incidence of intraventricular haemorrhage (IVH) among preterm infants (less than 37 weeks gestational age) has remained relatively constant (15.2 per cent in 2000). In 2000, confirmed IVH was most common among infants less than 28-weeks gestation (38.2 per cent); 38.0 per cent of these infants had severe IVH (grade 3 or 4 ). Two infants less than 32 weeks gestation with severe IVH required surgical drainage for post haemorrhagic hydrocephalus $(2 / 55 ; 3.6$ per cent). Of the surviving infants born before 32 weeks gestation, 94.0 per cent had a head ultrasound examination to detect IVH (Table 87). The incidence of severe IVH has remained constant since 1992.

The proportion of infants with severe grades (Grade 3 or Grade 4) of retinopathy of prematurity (ROP) has decreased from 7.5 per cent in 1992 to 4.8 per cent in 2000. In 2000, all infants with severe grades of ROP were less than 32 weeks gestation and 75.0 per cent of the infants less than 28 weeks gestation with severe ROP received either cryo- or laser therapy. Importantly, 20.5 per cent of surviving infants of 28-31 weeks gestational age did not have an eye examination recorded (Table 88).

\section{TABLE 86}

MAJOR SURGERY BY GESTATIONAL AGE, NSW \& ACT 2000\#

\begin{tabular}{|c|c|c|c|c|c|c|c|c|c|c|}
\hline \multirow[t]{3}{*}{ Major Surgery } & \multicolumn{10}{|c|}{ Gestational age (weeks) } \\
\hline & \multicolumn{2}{|c|}{ 23-27 } & \multicolumn{2}{|c|}{$28-31$} & \multicolumn{2}{|c|}{$32-36$} & \multicolumn{2}{|c|}{$37+$} & \multicolumn{2}{|c|}{ TOTAL } \\
\hline & No. & $\%$ & No. & $\%$ & No. & $\%$ & No. & $\%$ & No. & $\%$ \\
\hline No & 247 & 94.3 & 562 & 98.6 & 518 & 98.5 & 292 & 93.9 & 1619 & 97.0 \\
\hline Yes & 15 & 5.7 & 8 & 1.4 & 8 & 1.5 & 19 & 6.1 & 50 & 3.0 \\
\hline TOTAL & 262 & 100.0 & 570 & 100.0 & 526 & 100.0 & 311 & 100.0 & 1669 & 100.0 \\
\hline
\end{tabular}

Source: NICUS Data Collection. NSW Centre for Perinatal Health Services Research.

\# Babies with major congenital anomalies excluded.

\section{TABLE 87}

INTRAVENTRICULAR HAEMORRHAGE (IVH) BY GESTATIONAL AGE, NSW \& ACT 2000\#

\begin{tabular}{|c|c|c|c|c|c|c|c|c|}
\hline \multirow[t]{3}{*}{ Head ultrasound } & \multicolumn{8}{|c|}{ Gestational age (weeks) } \\
\hline & \multicolumn{2}{|c|}{ 23-27 } & \multicolumn{2}{|c|}{ 28-31 } & \multicolumn{2}{|c|}{$32-36$} & \multicolumn{2}{|c|}{ TOTAL } \\
\hline & No. & $\%$ & No. & $\%$ & No. & $\%$ & No. & $\%$ \\
\hline No IVH & 152 & 58.0 & 427 & 74.9 & 237 & 45.1 & 816 & 60.1 \\
\hline Grade 1 & 34 & 13.0 & 64 & 11.2 & 10 & 1.9 & 108 & 8.0 \\
\hline Grade 2 & 28 & 10.7 & 10 & 1.8 & 3 & 0.6 & 41 & 3.0 \\
\hline Grade 3 & 16 & 6.1 & 6 & 1.1 & 1 & 0.2 & 23 & 1.7 \\
\hline Grade 4 & 22 & 8.4 & 11 & 1.9 & 1 & 0.2 & 34 & 2.5 \\
\hline Hydrocephalus requiring drainage & 0 & 0.0 & 2 & 11.8 & 0 & 0.0 & 2 & 3.5 \\
\hline Not examined \& lived & 0 & 0.0 & 49 & 8.6 & 267 & 50.8 & 316 & 23.3 \\
\hline Not examined \& died & 10 & 3.8 & 3 & 0.5 & 7 & 1.3 & 20 & 1.5 \\
\hline TOTAL & 262 & 100.0 & 570 & 100.0 & 526 & 100.0 & 1358 & 100.0 \\
\hline
\end{tabular}

Source: NICUS Data Collection. NSW Centre for Perinatal Health Services Research.

\# Babies with major congenital anomalies excluded.

\section{TABLE 88}

RETINOPATHY OF PREMATURITY BY GESTATIONAL AGE, NSW \& ACT $2000^{\#}$

\begin{tabular}{|c|c|c|c|c|c|c|}
\hline \multirow[t]{2}{*}{ Retinopathy of prematurity (ROP) } & \multicolumn{6}{|c|}{ Gestational age (weeks) } \\
\hline & No. & $\%$ & No. & $\%$ & No. & $\%$ \\
\hline No ROP & 94 & 35.9 & 402 & 70.5 & 496 & 59.6 \\
\hline Grade 1 & 26 & 9.9 & 24 & 4.2 & 50 & 6.0 \\
\hline Grade 2 & 41 & 15.6 & 11 & 1.9 & 52 & 6.3 \\
\hline Grade 3 & 34 & 13.0 & 4 & 0.7 & 38 & 4.6 \\
\hline Grade 4 & 2 & 0.8 & 0 & 0.0 & 2 & 0.2 \\
\hline Treatment with cryotherapy-laser & 27 & 75.0 & 3 & 75.0 & 30 & 75.0 \\
\hline Not examined \& lived & 2 & 0.8 & 114 & 20.0 & 116 & 13.9 \\
\hline Not examined \& died & 63 & 24.0 & 15 & 2.6 & 78 & 9.4 \\
\hline TOTAL & 262 & 100.0 & 570 & 100.0 & 832 & 100.0 \\
\hline
\end{tabular}

Source: NICUS Data Collection. NSW Centre for Perinatal Health Services Research.

\# Babies with major congenital anomalies excluded. 


\subsection{SERVICE UTILISATION}

Indicators of service utilisation collected as part of NICUS include length of stay in tertiary and non-tertiary centres, days on assisted ventilation and days in oxygen (Figures 10 and 11 and Table 89). On an individual basis, infants born at less than 28 weeks gestation consumed most resources. However, as a group those born at 28-31 weeks gestation consumed more bed days than any other group due to their higher numbers. In 2000, the total cohort used 58,529 bed days in a tertiary centre in NSW and the ACT (range 46,090 in 1993 to 58,529 in 2000); as well as 18,411 in a non-tertiary centre (level 2 neonatal unit) in NSW and the ACT $(14,288$ in 1992 to 19,134 in 1999). Even when these infants leave the neonatal intensive care unit, they still require substantial resources.

In 2000, NICUS registrants used 18,909 days of assisted ventilation (range 15,282 in 1993 to 18,070 in 1998) and 29,998 days of oxygen therapy (range 22,526 in 1992 to 30,419 in 1998). The overall proportion of infants going home on supplemental oxygen was 4.5 per cent in 2000 (range 2.1 per cent in 1992 to 5.1 per cent in 1998). The proportion of infants less than 28 weeks gestation going home on supplemental oxygen was 18.7 per cent (range 7.5 per cent in 1992 to 20.0 per cent in 1998) (Table 90).

\section{FIGURE 10}

MEDIAN NUMBER OF DAYS IN HOSPITAL, OXYGEN THERAPY AND ASSISTED VENTILATION BY GESTATIONAL AGE, NSW \& ACT 2000

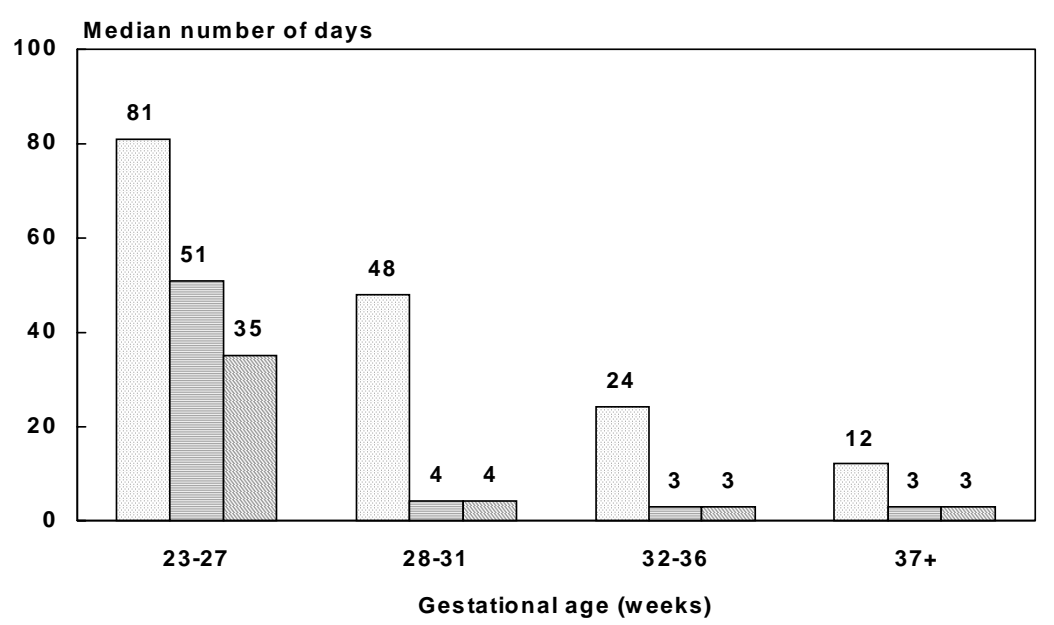

$\square$ Total length of stay $\square$ Days of oxygen therapy $\square$ Days of assisted ventilation

Source: NICUS Data Collection. NSW Centre for Perinatal Health Services Research.

FIGURE 11

TOTAL NUMBER OF DAYS IN HOSPITAL, OXYGEN THERAPY AND ASSISTED VENTILATION BY GESTATIONAL AGE, NSW \& ACT 2000

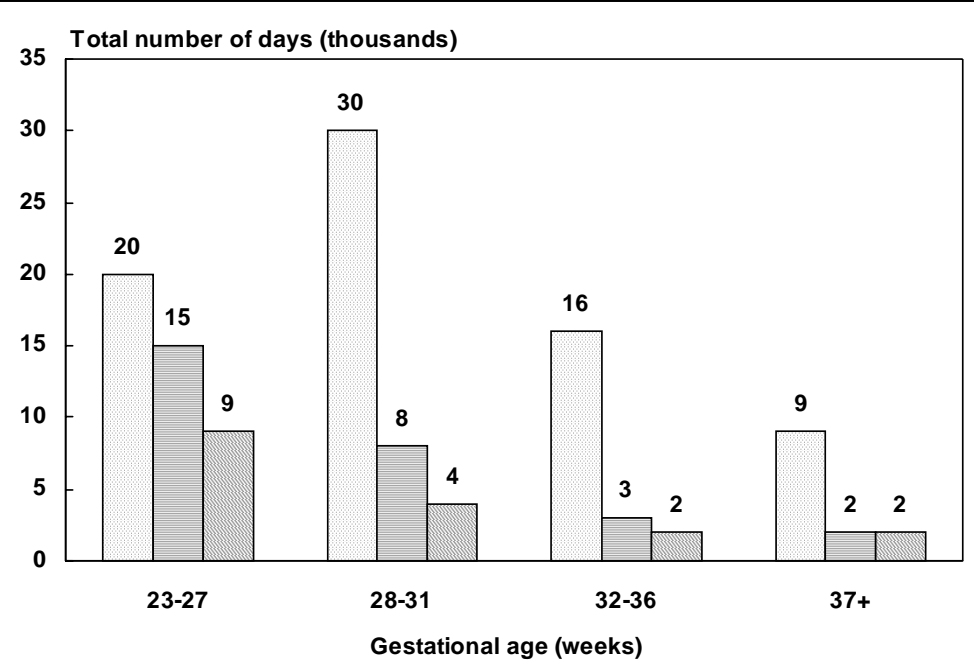

$\square$ Total length of stay $\square$ Days of oxygen therapy $\square$ Days of assisted ventilation

Source: NICUS Data Collection. NSW Centre for Perinatal Health Services Research. 


\section{TABLE 89}

SERVICE UTILISATION INDICATORS BY GESTATIONAL AGE, NSW \& ACT 2000

\begin{tabular}{|c|c|c|c|c|c|}
\hline \multirow{2}{*}{ Indicators } & \multicolumn{5}{|c|}{ Gestational age (weeks) } \\
\hline & 23-27 & 28-31 & $32-36$ & $37+$ & TOTAL \\
\hline \multicolumn{6}{|c|}{ Non-tertiary hospital stay (days) } \\
\hline Minimum & 0 & 0 & 0 & 0 & 0 \\
\hline Maximum & 109 & 230 & 60 & 77 & 230 \\
\hline Sum & 2527 & 9473 & 5036 & 1370 & 18411 \\
\hline Median & 0 & 13 & 3 & 0 & 1 \\
\hline 25th percentile & 0 & 0 & 0 & 0 & 0 \\
\hline 75th percentile & 17 & 28 & 15 & 3 & 16 \\
\hline \multicolumn{6}{|c|}{ Tertiary hospital stay (days) } \\
\hline Minimum & 1 & 1 & 1 & 1 & 1 \\
\hline Maximum & 292 & 163 & 321 & 350 & 350 \\
\hline Sum & 18016 & 21358 & 11101 & 8054 & 58529 \\
\hline Median & 69 & 32 & 13 & 9 & 18 \\
\hline 25th percentile & 29 & 17 & 8 & 6 & 8 \\
\hline 75th percentile & 96 & 49 & 24 & 17 & 40 \\
\hline \multicolumn{6}{|c|}{ Total hospital stay (days) } \\
\hline Minimum & 1 & 1 & 1 & 1 & 1 \\
\hline Maximum & 292 & 245 & 321 & 355 & 355 \\
\hline Sum & 20543 & 30831 & 16137 & 9429 & 76940 \\
\hline Median & 81 & 48 & 24 & 12 & 30 \\
\hline 25th percentile & 44 & 38 & 16 & 7 & 14 \\
\hline 75th percentile & 103 & 62 & 33 & 21 & 53 \\
\hline \multicolumn{6}{|c|}{ Mechanical ventilation (days) } \\
\hline Minimum & 0 & 0 & 0 & 0 & 0 \\
\hline Maximum & 181 & 74 & 155 & 44 & 181 \\
\hline Sum & 4248 & 1536 & 1317 & 1831 & 8932 \\
\hline Median & 8 & 0 & 1 & 2 & 2 \\
\hline 25th percentile & 2 & 0 & 0 & 1 & 0 \\
\hline 75th percentile & 22 & 3 & 3 & 5 & 4 \\
\hline \multicolumn{6}{|c|}{ Continuous positive airways pressure (days) } \\
\hline Minimum & 0 & 0 & 0 & 0 & 0 \\
\hline Maximum & 84 & 81 & 45 & 37 & 84 \\
\hline Sum & 5562 & 3089 & 890 & 436 & 9977 \\
\hline Median & 19 & 2 & 1 & 0 & 1 \\
\hline 25th percentile & 1 & 0 & 0 & 0 & 0 \\
\hline 75th percentile & 33 & 6 & 2 & 1 & 4 \\
\hline \multicolumn{6}{|c|}{ Assisted ventilation (days) } \\
\hline Minimum & 0 & 0 & 0 & 0 & 0 \\
\hline Maximum & 265 & 81 & 156 & 67 & 265 \\
\hline Sum & 9810 & 4625 & 2207 & 2267 & 18909 \\
\hline Median & 35 & 4 & 3 & 3 & 3 \\
\hline 25th percentile & 13 & 1 & 1 & 1 & 1 \\
\hline 75th percentile & 51 & 9 & 5 & 5 & 8 \\
\hline \multicolumn{6}{|l|}{ Oxygen (days) } \\
\hline Minimum & 0 & 0 & 0 & 0 & 0 \\
\hline Maximum & 292 & 131 & 156 & 51 & 292 \\
\hline Sum & 15401 & 8353 & 3380 & 2864 & 29998 \\
\hline Median & 51 & 4 & 3 & 3 & 4 \\
\hline 25th percentile & 10 & 1 & 1 & 1 & 1 \\
\hline 75th percentile & 91 & 16 & 6 & 7 & 12 \\
\hline
\end{tabular}

Source: NICUS Data Collection. NSW Centre for Perinatal Health Services Research. 


\begin{tabular}{|c|c|c|c|c|c|c|c|c|c|c|c|}
\hline \multirow[t]{3}{*}{ Year } & \multirow{3}{*}{ Home oxygen } & \multicolumn{10}{|c|}{ Gestational age (weeks) } \\
\hline & & \multicolumn{2}{|c|}{$23-27$} & \multicolumn{2}{|c|}{$28-31$} & \multicolumn{2}{|c|}{$32-36$} & \multicolumn{2}{|c|}{$37+$} & \multicolumn{2}{|c|}{ TOTAL } \\
\hline & & No. & $\%$ & No. & $\%$ & No. & $\%$ & No. & $\%$ & No. & $\%$ \\
\hline \multirow{3}{*}{1996} & No & 191 & 83.8 & 483 & 96.2 & 469 & 99.4 & 267 & 97.8 & 1410 & 95.6 \\
\hline & Yes & 37 & 16.2 & 19 & 3.8 & 3 & 0.6 & 6 & 2.2 & 65 & 4.4 \\
\hline & TOTAL & 228 & 100.0 & 502 & 100.0 & 472 & 100.0 & 273 & 100.0 & 1475 & 100.0 \\
\hline \multirow[t]{3}{*}{1997} & No & 204 & 89.9 & 498 & 96.3 & 464 & 99.6 & 254 & 98.8 & 1420 & 96.8 \\
\hline & Yes & 23 & 10.1 & 19 & 3.7 & 2 & 0.4 & 3 & 1.2 & 47 & 3.2 \\
\hline & TOTAL & 227 & 100.0 & 517 & 100.0 & 466 & 100.0 & 257 & 100.0 & 1467 & 100.0 \\
\hline \multirow[t]{3}{*}{1998} & No & 224 & 80.0 & 550 & 97.5 & 459 & 99.1 & 266 & 97.8 & 1499 & 94.9 \\
\hline & Yes & 56 & 20.0 & 14 & 2.5 & 4 & 0.9 & 6 & 2.2 & 80 & 5.1 \\
\hline & TOTAL & 280 & 100.0 & 564 & 100.0 & 463 & 100.0 & 272 & 100.0 & 1579 & 100.0 \\
\hline \multirow[t]{3}{*}{1999} & No & 243 & 86.5 & 512 & 97.7 & 521 & 99.4 & 298 & 99.0 & 1574 & 96.6 \\
\hline & Yes & 38 & 13.5 & 12 & 2.3 & 3 & 0.6 & 3 & 1.0 & 56 & 3.4 \\
\hline & TOTAL & 281 & 100.0 & 524 & 100.0 & 524 & 100.0 & 301 & 100.0 & 1630 & 100.0 \\
\hline \multirow[t]{3}{*}{2000} & No & 213 & 81.3 & 555 & 97.4 & 519 & 98.7 & 307 & 98.7 & 1594 & 95.5 \\
\hline & Yes & 49 & 18.7 & 15 & 2.6 & 7 & 1.3 & 4 & 1.3 & 75 & 4.5 \\
\hline & TOTAL & 262 & 100.0 & 570 & 100.0 & 526 & 100.0 & 311 & 100.0 & 1669 & 100.0 \\
\hline
\end{tabular}

Source: NICUS Data Collection. NSW Centre for Perinatal Health Services Research.

\# Babies with major congenital anomalies excluded.

\section{TABLE 91}

DURATION OF SURVIVAL OF BABIES BY GESTATIONAL AGE, NSW \& ACT 2000*

\begin{tabular}{|c|c|c|c|c|c|c|c|c|c|c|}
\hline \multirow{2}{*}{$\begin{array}{l}\text { Gestational age } \\
\text { (weeks) }\end{array}$} & \multicolumn{2}{|c|}{$\begin{array}{l}\text { Alive at six } \\
\text { months }\end{array}$} & \multicolumn{6}{|c|}{ Age at death (days) } & \multicolumn{2}{|c|}{ TOTAL } \\
\hline & No. & $\%$ & No. & $\%$ & No. & $\%$ & No. & $\%$ & No. & $\%$ \\
\hline 23 & 6 & 35.3 & 6 & 35.3 & 2 & 11.8 & 3 & 17.6 & 17 & 1.0 \\
\hline 24 & 26 & 54.2 & 12 & 25.0 & 4 & 8.3 & 6 & 12.5 & 48 & 2.9 \\
\hline 25 & 20 & 52.6 & 10 & 26.3 & 6 & 15.8 & 2 & 5.3 & 38 & 2.3 \\
\hline 26 & 61 & 85.9 & 9 & 12.7 & 1 & 1.4 & 0 & 0.0 & 71 & 4.3 \\
\hline 27 & 80 & 90.9 & 6 & 6.8 & 2 & 2.3 & 0 & 0.0 & 88 & 5.3 \\
\hline 28 & 109 & 94.0 & 3 & 2.6 & 3 & 2.6 & 1 & 0.9 & 116 & 7.0 \\
\hline 29 & 122 & 94.6 & 6 & 4.7 & 0 & 0.0 & 1 & 0.8 & 129 & 7.7 \\
\hline 30 & 141 & 97.9 & 1 & 0.7 & 1 & 0.7 & 1 & 0.7 & 144 & 8.6 \\
\hline 31 & 180 & 99.4 & 1 & 0.6 & 0 & 0.0 & 0 & 0.0 & 181 & 10.8 \\
\hline 32 & 135 & 99.3 & 0 & 0.0 & 0 & 0.0 & 1 & 0.7 & 136 & 8.1 \\
\hline 33 & 136 & 97.8 & 3 & 2.2 & 0 & 0.0 & 0 & 0.0 & 139 & 8.3 \\
\hline 34 & 106 & 97.2 & 2 & 1.8 & 0 & 0.0 & 1 & 0.9 & 109 & 6.5 \\
\hline 35 & 75 & 97.4 & 2 & 2.6 & 0 & 0.0 & 0 & 0.0 & 77 & 4.6 \\
\hline 36 & 63 & 95.5 & 3 & 4.5 & 0 & 0.0 & 0 & 0.0 & 66 & 4.0 \\
\hline 37 & 49 & 94.2 & 3 & 5.8 & 0 & 0.0 & 0 & 0.0 & 52 & 3.1 \\
\hline 38 & 69 & 92.0 & 5 & 6.7 & 0 & 0.0 & 1 & 1.3 & 75 & 4.5 \\
\hline 39 & 58 & 92.1 & 3 & 4.8 & 2 & 3.2 & 0 & 0.0 & 63 & 3.8 \\
\hline 40 & 57 & 89.1 & 6 & 9.4 & 0 & 0.0 & 1 & 1.6 & 64 & 3.8 \\
\hline 41 & 44 & 88.0 & 4 & 8.0 & 1 & 2.0 & 1 & 2.0 & 50 & 3.0 \\
\hline 42 & 7 & 100.0 & 0 & 0.0 & 0 & 0.0 & 0 & 0.0 & 7 & 0.4 \\
\hline TOTAL & 1543 & 92.5 & 85 & 5.1 & 22 & 1.3 & 19 & 1.1 & 1669 & 100.0 \\
\hline
\end{tabular}

Source: NICUS Data Collection. NSW Centre for Perinatal Health Services Research.

\# Babies with major congenital anomalies excluded. 


\subsection{SURVIVAL}

Infants with a major congenital anomaly have been excluded from the analysis of survival with the exception of data reported in Table 94.

The six-month survival rate for all infants without a major congenital anomaly in the 2000 cohort was 92.5 per cent compared with 87.8 per cent in 1992. Survival of infants born at less than 25 weeks gestation was 49.2 per cent (range 33.9 per cent in 1998 to 54.8 per cent in 1993). There was a trend for survival to improve with gestational age up to 32 weeks gestation after which it decreased slightly. Term infants ( 91.3 per cent) were slightly less likely to survive than preterm infants (92.7 per cent). Amongst infants who died, 67.5 per cent of deaths occurred during the first week of life (compared with 62.5 per cent in 1998 to 75.5 per cent in 1994) with a further 17.5 per cent occurring during the first month of life (Table 91).

The six-month survival rate improved with increasing birth weight, ranging from 35.3 per cent for infants in the 500599 gram group to 86.1 per cent for the 900-999 gram group. Six-month survival continued to improve with increasing birthweight to a maximum of 98.9 per cent for infants of 1,500-1,749 grams birthweight and then decreased slightly (Table 92).

The majority of infants registered in NICUS were born at a tertiary centre. Although the gestational age is the most important risk factor for mortality, disease severity is also important. At each gestational age group those with severe disease are more likely to be transferred to a neonatal intensive care unit.

In 2000, the six-month survival rate for infants born at 23 to 27 weeks was greater for those born in a non-tertiary centre ( 90.5 per cent; $n=21$ ) compared with those born in a tertiary centre ( 73.1 per cent; $n=234$ ). These results should be interpreted with extreme caution. Of the surviving infants born in a non-tertiary centre $11 / 19$ were 27 weeks gestation. Place of birth did not significantly affect survival for infants in the other gestational age groups (Table 93).

The six-month survival rate for male infants ( 92.5 per cent) was similar to that for female infants (92.4 per cent). The six-month survival rate was similar for males and females for all gestational age groups: less than 28 weeks ( 70.8 per cent versus 77.1 per cent), 28-31 weeks (95.9 per cent versus 98.0 per cent), 32-36 weeks (97.8 per cent versus 97.6 per cent) and $37-41$ weeks gestation groups (94.3per cent versus 85.5 per cent).

The six-month survival rate was 92.6 per cent $(n=1,276)$ for singleton infants and 92.1 per cent $(n=393)$ for multiple gestation infants. Plurality did not influence survival in infants greater than 28 weeks gestational age. In 2000 the survival rate for infants in the less than 28 week gestation group was lower for infants born of a multiple (44/66; 66.7 per cent) than a singleton pregnancy (149/ 196; 76.0 per cent).

As expected survival was generally lower (83.5 per cent) in the presence of a major congenital anomaly (Table 94).

Post-mortem examinations were performed on 39/126 infants (31.0 per cent) who died in the 2000 cohort (Figure 12 and Table 95). Post-mortem examinations were most commonly not requested for infants $23-27$ weeks gestation (55.1 per cent) and 28-31weeks gestation (55.6 per cent). The highest rate of refusal was in term group (22.2 per cent) and the highest rate of post-mortems done was in the term group ( 48.1 per cent).

\section{TABLE 92}

DURATION OF SURVIVAL BY BIRTHWEIGHT, NSW \& ACT 2000\#

\begin{tabular}{|c|c|c|c|c|c|c|c|c|c|c|}
\hline \multirow[t]{3}{*}{ Birthweight (grams) } & \multirow{2}{*}{\multicolumn{2}{|c|}{$\begin{array}{l}\text { Alive at six } \\
\text { months }\end{array}$}} & \multicolumn{6}{|c|}{ Age at death (days) } & \multirow{2}{*}{\multicolumn{2}{|c|}{ TOTAL }} \\
\hline & & & & & & & & & & \\
\hline & No. & $\%$ & No. & $\%$ & No. & $\%$ & No. & $\%$ & No. & $\%$ \\
\hline Less than 400 & 0 & 0.0 & 1 & 100.0 & 0 & 0.0 & 0 & 0.0 & 1 & 0.1 \\
\hline $400-499$ & 1 & 16.7 & 3 & 50.0 & 1 & 16.7 & 1 & 16.7 & 6 & 0.4 \\
\hline $500-599$ & 6 & 35.3 & 6 & 35.3 & 1 & 5.9 & 4 & 23.5 & 17 & 1.0 \\
\hline $600-699$ & 34 & 63.0 & 14 & 25.9 & 3 & 5.6 & 3 & 5.6 & 54 & 3.2 \\
\hline $700-799$ & 42 & 70.0 & 12 & 20.0 & 4 & 6.7 & 2 & 3.3 & 60 & 3.6 \\
\hline $800-899$ & 42 & 87.5 & 3 & 6.3 & 3 & 6.3 & 0 & 0.0 & 48 & 2.9 \\
\hline $900-999$ & 68 & 86.1 & 6 & 7.6 & 4 & 5.1 & 1 & 1.3 & 79 & 4.7 \\
\hline $1,000-1,249$ & 193 & 96.5 & 6 & 3.0 & 1 & 0.5 & 0 & 0.0 & 200 & 12.0 \\
\hline $1,250-1,499$ & 254 & 97.7 & 1 & 0.4 & 1 & 0.4 & 4 & 1.5 & 260 & 15.6 \\
\hline $1,500-1,749$ & 187 & 98.9 & 1 & 0.5 & 1 & 0.5 & 0 & 0.0 & 189 & 11.3 \\
\hline $1,750-1,999$ & 130 & 97.7 & 3 & 2.3 & 0 & 0.0 & 0 & 0.0 & 133 & 8.0 \\
\hline $2,000-2,499$ & 201 & 96.2 & 7 & 3.3 & 0 & 0.0 & 1 & 0.5 & 209 & 12.5 \\
\hline $2,500-2,999$ & 126 & 94.0 & 8 & 6.0 & 0 & 0.0 & 0 & 0.0 & 134 & 8.0 \\
\hline $3,000-3,499$ & 120 & 93.0 & 7 & 5.4 & 0 & 0.0 & 2 & 1.6 & 129 & 7.7 \\
\hline $3,500-3,999$ & 86 & 91.5 & 4 & 4.3 & 3 & 3.2 & 1 & 1.1 & 94 & 5.6 \\
\hline $4,000+$ & 53 & 94.6 & 3 & 5.4 & 0 & 0.0 & 0 & 0.0 & 56 & 3.4 \\
\hline TOTAL & 1543 & 92.5 & 85 & 5.1 & 22 & 1.3 & 19 & 1.1 & 1669 & 100.0 \\
\hline
\end{tabular}

Source: NICUS Data Collection. NSW Centre for Perinatal Health Services Research.

\# Babies with major congenital anomalies excluded. 


\begin{tabular}{|c|c|c|c|c|c|c|c|c|c|c|c|}
\hline \multirow{2}{*}{$\begin{array}{l}\text { Gestational age } \\
\text { (weeks) }\end{array}$} & \multirow[t]{2}{*}{ Place of birth } & \multicolumn{2}{|c|}{$\begin{array}{l}\text { Alive at six } \\
\text { months }\end{array}$} & \multicolumn{2}{|c|}{$0-7$} & \multicolumn{2}{|c|}{ Age at death (days) } & \multicolumn{2}{|c|}{ 28+ } & \multicolumn{2}{|c|}{ TOTAL } \\
\hline & & No. & $\begin{array}{l}\text { iths } \\
\%\end{array}$ & No. & $\%$ & No. & $\%$ & No. & $\%$ & No. & $\%$ \\
\hline \multirow[t]{3}{*}{ 23-27 } & Non tertiary & 19 & 90.5 & 2 & 9.5 & 0 & 0.0 & 0 & 0.0 & 21 & 8.2 \\
\hline & Tertiary & 171 & 73.1 & 39 & 16.7 & 13 & 5.6 & 11 & 4.7 & 234 & 91.8 \\
\hline & Sub-total & 190 & 74.5 & 41 & 16.1 & 13 & 5.1 & 11 & 4.3 & 255 & 100.0 \\
\hline \multirow[t]{3}{*}{$28-31$} & Non tertiary & 42 & 100.0 & 0 & 0.0 & 0 & 0.0 & 0 & 0.0 & 42 & 7.4 \\
\hline & Tertiary & 509 & 96.6 & 11 & 2.1 & 4 & 0.8 & 3 & 0.6 & 527 & 92.6 \\
\hline & Sub-total & 551 & 96.8 & 11 & 1.9 & 4 & 0.7 & 3 & 0.5 & 569 & 100.0 \\
\hline \multirow[t]{3}{*}{$32-36$} & Non tertiary & 138 & 97.2 & 4 & 2.8 & 0 & 0.0 & 0 & 0.0 & 142 & 27.0 \\
\hline & Tertiary & 376 & 97.9 & 6 & 1.6 & 0 & 0.0 & 2 & 0.5 & 384 & 73.0 \\
\hline & Sub-total & 514 & 97.7 & 10 & 1.9 & 0 & 0.0 & 2 & 0.4 & 526 & 100.0 \\
\hline \multirow[t]{3}{*}{$37-41$} & Non tertiary & 132 & 86.3 & 16 & 10.5 & 3 & 2.0 & 2 & 1.3 & 153 & 50.7 \\
\hline & Tertiary & 144 & 96.6 & 4 & 2.7 & 0 & 0.0 & 1 & 0.7 & 149 & 49.3 \\
\hline & Sub-total & 276 & 91.4 & 20 & 6.6 & 3 & 1.0 & 3 & 1.0 & 302 & 100.0 \\
\hline \multirow[t]{3}{*}{$42+$} & Non tertiary & 4 & 100.0 & 0 & 0.0 & 0 & 0.0 & 0 & 0.0 & 4 & 57.1 \\
\hline & Tertiary & 3 & 100.0 & 0 & 0.0 & 0 & 0.0 & 0 & 0.0 & 3 & 42.9 \\
\hline & Sub-total & 7 & 100.0 & 0 & 0.0 & 0 & 0.0 & 0 & 0.0 & 7 & 100.0 \\
\hline TOTAL & & 1538 & 92.7 & 82 & 4.9 & 20 & 1.2 & 19 & 1.1 & 1659 & 100.0 \\
\hline
\end{tabular}

Source: NICUS Data Collection. NSW Centre for Perinatal Health Services Research.

\# Babies with major congenital anomalies excluded. Babies born before arrival excluded.

\section{TABLE 94}

DURATION OF SURVIVAL BY MAJOR CONGENITAL ANOMALY AND GESTATIONAL AGE, NSW \& ACT 2000

\begin{tabular}{|c|c|c|c|c|c|c|c|c|c|c|c|}
\hline \multirow{3}{*}{$\begin{array}{l}\text { Gestational age } \\
\text { (weeks) }\end{array}$} & \multirow{3}{*}{$\begin{array}{l}\text { Major congenital } \\
\text { anomaly }\end{array}$} & \multirow{2}{*}{\multicolumn{2}{|c|}{$\begin{array}{l}\text { Alive at six } \\
\text { months }\end{array}$}} & \multirow{2}{*}{\multicolumn{2}{|c|}{$0-7$}} & \multicolumn{4}{|c|}{ Age at death (days) } & \multicolumn{2}{|c|}{ TOTAL } \\
\hline & & & & & & & & & & & \\
\hline & & No. & $\%$ & No. & $\%$ & No. & $\%$ & No. & $\%$ & No. & $\%$ \\
\hline \multirow[t]{3}{*}{$23-27$} & No & 193 & 73.7 & 43 & 16.4 & 15 & 5.7 & 11 & 4.2 & 262 & 95.3 \\
\hline & Yes & 8 & 61.5 & 3 & 23.1 & 2 & 15.4 & 0 & 0.0 & 13 & 4.7 \\
\hline & Sub-total & 201 & 73.1 & 46 & 16.7 & 17 & 6.2 & 11 & 4.0 & 275 & 100.0 \\
\hline \multirow[t]{3}{*}{$28-31$} & No & 552 & 96.8 & 11 & 1.9 & 4 & 0.7 & 3 & 0.5 & 570 & 94.2 \\
\hline & Yes & 25 & 71.4 & 4 & 11.4 & 1 & 2.9 & 5 & 14.3 & 35 & 5.8 \\
\hline & Sub-total & 577 & 95.4 & 15 & 2.5 & 5 & 0.8 & 8 & 1.3 & 605 & 100.0 \\
\hline \multirow[t]{3}{*}{$32-36$} & No & 514 & 97.7 & 10 & 1.9 & 0 & 0.0 & 2 & 0.4 & 526 & 87.5 \\
\hline & Yes & 57 & 76.0 & 12 & 16.2 & 2 & 2.7 & 4 & 5.4 & 75 & 12.5 \\
\hline & Sub-total & 571 & 95.0 & 22 & 3.7 & 2 & 0.3 & 6 & 1.0 & 601 & 100.0 \\
\hline \multirow[t]{3}{*}{$37-41$} & No & 277 & 91.1 & 21 & 6.9 & 3 & 1.0 & 3 & 1.0 & 304 & 59.4 \\
\hline & Yes & 187 & 89.9 & 10 & 4.8 & 6 & 2.9 & 5 & 2.4 & 208 & 40.6 \\
\hline & Sub-total & 464 & 90.6 & 31 & 6.1 & 9 & 1.8 & 8 & 1.6 & 512 & 100.0 \\
\hline \multirow[t]{3}{*}{$42+$} & No & 7 & 100.0 & 0 & 0.0 & 0 & 0.0 & 0 & 0.0 & 7 & 70.0 \\
\hline & Yes & 2 & 66.7 & 0 & 0.0 & 0 & 0.0 & 1 & 33.3 & 3 & 30.0 \\
\hline & Sub-total & 9 & 90.0 & 0 & 0.0 & 0 & 0.0 & 1 & 10.0 & 10 & 100.0 \\
\hline TOTAL & & 1822 & 91.0 & 114 & 5.7 & 33 & 1.6 & 34 & 1.7 & 2003 & 100.0 \\
\hline
\end{tabular}

Source: NICUS Data Collection. NSW Centre for Perinatal Health Services Research. 


\section{FIGURE 12}

DEATHS BY POST-MORTEM EXAMINATION AND GESTATIONAL AGE, NSW \& ACT 1996-2000\#

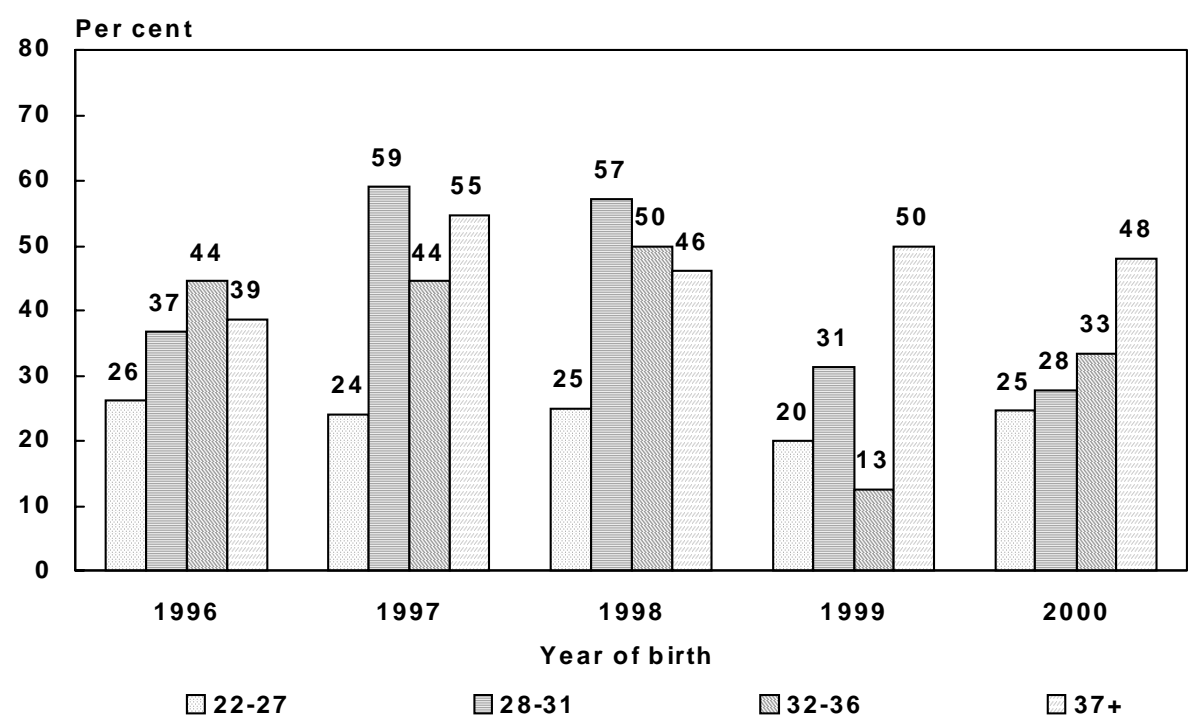

Source: NICUS Data Collection. NSW Centre for Perinatal Health Services Research.

\# Infants with major congenital anomalies excluded.

\section{TABLE 95}

POST-MORTEM EXAMINATION BY GESTATIONAL AGE, NSW \& ACT 2000\#

\begin{tabular}{|c|c|c|c|c|c|c|c|c|c|c|}
\hline \multirow[t]{3}{*}{ Post-mortem } & \multicolumn{10}{|c|}{ Gestational age (weeks) } \\
\hline & \multicolumn{2}{|c|}{ 23-27 } & \multicolumn{2}{|c|}{$28-31$} & \multicolumn{2}{|c|}{$32-36$} & \multicolumn{2}{|c|}{$37+$} & \multicolumn{2}{|c|}{ TOTAL } \\
\hline & No. & $\%$ & No. & $\%$ & No. & $\%$ & No. & $\%$ & No. & $\%$ \\
\hline Not requested & 38 & 55.1 & 10 & 55.6 & 6 & 50.0 & 8 & 29.6 & 62 & 49.2 \\
\hline Refused & 14 & 20.3 & 3 & 16.7 & 2 & 16.7 & 6 & 22.2 & 25 & 19.8 \\
\hline Done & 17 & 24.6 & 5 & 27.8 & 4 & 33.3 & 13 & 48.1 & 39 & 31.0 \\
\hline TOTAL & 69 & 100.0 & 18 & 100.0 & 12 & 100.0 & 27 & 100.0 & 126 & 100.0 \\
\hline
\end{tabular}

Source: NICUS Data Collection. NSW Centre for Perinatal Health Services Research.

\# Babies with major congenital anomalies excluded. 


\section{PART 6: BIRTH DEFECTS}

\subsection{BIRTH DEFECTS AMONG STILLBORN AND LIVEBORN INFANTS}

A birth defect is any structural defect detected during pregnancy or at birth, excluding birth injuries and minor anomalies such as skin tags, talipes, birthmarks or clicky hips. Descriptions of some common birth defects are shown in Appendix 1. A list of common exclusions is shown in Appendix 2.

From 1 January 1998 doctors, hospitals and laboratories are required to notify birth defects detected during pregnancy, at birth, or up to one year of life under NSW Public Health Act 1991. Information reported is included in the NSW Birth Defects Register (BDR). The quality of information received by the BDR has improved since 1998, particularly in relation to pregnancy outcome.

This chapter reports birth defects detected during pregnancy or in the first year of life for 1994-1999 and birth defects detected during pregnancy or at birth for 2000.

\subsubsection{TRENDS IN REPORTED BIRTH DEFECTS}

Between 1994 and 1999, the reported number of infants with birth defects has remained stable at just over two per cent (Table 96). In 2000, 1056 cases of birth defects detected during pregnancy or at birth were reported.

\subsubsection{BIRTH DEFECTS BY DIAGNOSTIC CATEGORY}

The most common categories of birth defects for births of more than 20 weeks gestation or with a birthweight greater than 400 grams are presented in Table 97. Birth defects

\section{TABLE 96}

BIRTH DEFECT CASES, NSW 1994-2000"

\begin{tabular}{|cccc|}
\hline Year & Birth defect cases & Births & Rate/1,000 births \\
\hline 1994 & 1989 & 87262 & 22.8 \\
1995 & 1947 & 86648 & 22.5 \\
1996 & 1880 & 85706 & 21.9 \\
1997 & 2001 & 87416 & 22.9 \\
1998 & 1949 & 85627 & 22.8 \\
1999 & 1836 & 86468 & 21.2 \\
2000 & 1056 & 87279 & 12.1 \\
\hline
\end{tabular}

Source: NSW Birth Defects Register. Epidemiology and Surveillance Branch, NSW Department of Health.

\# $\quad$ For 1994-1999, cases reported during pregnancy and up to one year of age are included. For 2000, cases reported during pregnancy or at birth are reported.

are classified using the British Paediatric Association (BPA) Classification of Diseases, which is primarily organised by body system. ${ }^{1}$ For infants with more than one defect, each defect is counted separately. The number of birth defects reported therefore exceeds the number of affected infants.

In 1994-2000, defects of the cardiovascular system were most commonly reported, followed by defects of the musculoskeletal system and defects of the genito-urinary system (Table 97). This is a similar pattern to previous years. In 1999, the overall rate of defects was slightly lower than the previous five years combined (39.5 versus 42.9 per 1,000), due to a decrease in the number of babies with multiple malformations. The proportion of reported cases with three or more malformations fell from 21.4 to 18.9 per cent between 1994 and 1999.

\section{TABLE 97}

BIRTH DEFECTS AMONG STILLBIRTHS AND LIVE BIRTHS BY DIAGNOSTIC CATEGORY, NSW 1994-2000

\begin{tabular}{|c|c|c|c|c|c|c|c|c|}
\hline \multirow[t]{2}{*}{ Diagnostic category } & \multicolumn{4}{|c|}{ No. defects } & \multicolumn{4}{|c|}{ Rate/1,000 births } \\
\hline & 1994-1998 & 1999 & 2000 & 1994-2000 & 1994-1998 & 1999 & 2000 & 1994-2000 \\
\hline \multicolumn{9}{|l|}{ Defects of nervous system } \\
\hline Anencephaly & 51 & 5 & 9 & 65 & 0.1 & 0.1 & 0.1 & 0.1 \\
\hline Spina Bifida & 145 & 32 & 23 & 200 & 0.3 & 0.4 & 0.3 & 0.3 \\
\hline Encephalocele & 36 & 5 & 3 & 44 & 0.1 & 0.1 & 0.0 & 0.1 \\
\hline Microcephaly & 142 & 18 & 12 & 172 & 0.3 & 0.2 & 0.1 & 0.3 \\
\hline Congenital hydrocephalus & 183 & 37 & 32 & 252 & 0.4 & 0.4 & 0.4 & 0.4 \\
\hline Other nervous system defects & 413 & 79 & 45 & 537 & 1.0 & 0.9 & 0.5 & 0.9 \\
\hline TOTAL & 970 & 176 & 124 & 1270 & 2.2 & 2.0 & 1.4 & 2.1 \\
\hline \multicolumn{9}{|l|}{ Defects of eye } \\
\hline Anophthalmos/ microphthalmos & 74 & 18 & 8 & 100 & 0.2 & 0.2 & 0.1 & 0.2 \\
\hline Buphthalmos/ congenital glaucoma & 33 & 6 & 1 & 40 & 0.1 & 0.1 & 0.0 & 0.1 \\
\hline Congenital cataract & 88 & 23 & 4 & 115 & 0.2 & 0.3 & 0.0 & 0.2 \\
\hline Other eye defects & 198 & 37 & 12 & 247 & 0.5 & 0.4 & 0.1 & 0.4 \\
\hline TOTAL & 393 & 84 & 25 & 502 & 0.9 & 1.0 & 0.3 & 0.8 \\
\hline \multicolumn{9}{|l|}{ Defects of ear, face and neck } \\
\hline Absence/ stricture auditory canal & 44 & 10 & 7 & 61 & 0.1 & 0.1 & 0.1 & 0.1 \\
\hline Absent auricle & 6 & 1 & 3 & 10 & 0.0 & 0.0 & 0.0 & 0.0 \\
\hline Defects of face and neck & 44 & 4 & 5 & 53 & 0.1 & 0.0 & 0.1 & 0.1 \\
\hline Other ear defects & 93 & 11 & 13 & 117 & 0.2 & 0.1 & 0.1 & 0.2 \\
\hline TOTAL & 187 & 26 & 28 & 241 & 0.4 & 0.3 & 0.3 & 0.4 \\
\hline \multicolumn{9}{|l|}{ Defects of cardiovascular system } \\
\hline Transposition of great vessels & 217 & 37 & 29 & 283 & 0.5 & 0.4 & 0.3 & 0.5 \\
\hline Tetralogy of Fallot & 147 & 30 & 13 & 190 & 0.3 & 0.3 & 0.1 & 0.3 \\
\hline Ventricular septal defect & 1009 & 163 & 98 & 1270 & 2.3 & 1.9 & 1.1 & 2.1 \\
\hline Atrial septal defect & 1005 & 166 & 90 & 1261 & 2.3 & 1.9 & 1.0 & 2.1 \\
\hline Heart valve defects & 803 & 151 & 66 & 1020 & 1.9 & 1.7 & 0.8 & 1.7 \\
\hline
\end{tabular}




\section{TABLE 97 (CONT)}

BIRTH DEFECTS AMONG STILLBIRTHS AND LIVE BIRTHS BY DIAGNOSTIC CATEGORY, NSW 1994-2000\#

\begin{tabular}{|c|c|c|c|c|c|c|c|c|}
\hline \multirow[t]{2}{*}{ Diagnostic category } & \multicolumn{3}{|c|}{ No. defects } & \multirow[b]{2}{*}{ 1994-2000 } & \multirow[b]{2}{*}{ 1994-1998 } & \multicolumn{2}{|c|}{ Rate/1,000 births } & \multirow[b]{2}{*}{ 1994-2000 } \\
\hline & 994-1998 & 1999 & 2000 & & & 1999 & 2000 & \\
\hline \multicolumn{9}{|l|}{ Defects of cardiovascular system (cont.) } \\
\hline Patent ductus arteriosus $>37$ weeks & 608 & 106 & 54 & 768 & 1.4 & 1.2 & 0.6 & 1.3 \\
\hline Coarctation of aorta & 182 & 38 & 12 & 232 & 0.4 & 0.4 & 0.1 & 0.4 \\
\hline Other defects of aorta & 104 & 28 & 7 & 139 & 0.2 & 0.3 & 0.1 & 0.2 \\
\hline Defects of pulmonary artery & 137 & 29 & 13 & 179 & 0.3 & 0.3 & 0.1 & 0.3 \\
\hline Other cardiovascular defects & 887 & 199 & 96 & 1182 & 2.1 & 2.3 & 1.1 & 1.9 \\
\hline TOTAL & 5099 & 947 & 478 & 6524 & 11.8 & 11.0 & 5.5 & 10.8 \\
\hline \multicolumn{9}{|l|}{ Defects of respiratory system } \\
\hline Defects of nose & 77 & 16 & 10 & 103 & 0.2 & 0.2 & 0.1 & 0.2 \\
\hline Defects of larynx, trachea and bronchu & nus 50 & 8 & 4 & 62 & 0.1 & 0.1 & 0.0 & 0.1 \\
\hline Defects of lung & 97 & 26 & 10 & 133 & 0.2 & 0.3 & 0.1 & 0.2 \\
\hline Other respiratory defects & 3 & 0 & 0 & 3 & 0.0 & 0.0 & 0.0 & 0.0 \\
\hline TOTAL & 227 & 50 & 24 & 301 & 0.5 & 0.6 & 0.3 & 0.5 \\
\hline \multicolumn{9}{|l|}{ Defects of gastrointestinal system } \\
\hline Cleft palate only & 416 & 69 & 63 & 548 & 1.0 & 0.8 & 0.7 & 0.9 \\
\hline Cleft lip only & 181 & 30 & 27 & 238 & 0.4 & 0.3 & 0.3 & 0.4 \\
\hline Cleft palate and cleft lip & 261 & 46 & 38 & 345 & 0.6 & 0.5 & 0.4 & 0.6 \\
\hline Other gastrointestinal defects & 520 & 100 & 35 & 655 & 1.2 & 1.2 & 0.4 & 1.1 \\
\hline Oesophageal atresia only & 5 & 2 & 1 & 8 & 0.0 & 0.0 & 0.0 & 0.0 \\
\hline Oesophageal atresia with TOF & 81 & 24 & 10 & 115 & 0.2 & 0.3 & 0.1 & 0.2 \\
\hline Tracheo-oesophageal fistula (TOF) only & 27 & 5 & 7 & 39 & 0.1 & 0.1 & 0.1 & 0.1 \\
\hline Atresia-stenosis of small intestine & 127 & 29 & 21 & 177 & 0.3 & 0.3 & 0.2 & 0.3 \\
\hline Atresia-stenosis of anus & 158 & 36 & 14 & 208 & 0.4 & 0.4 & 0.2 & 0.3 \\
\hline TOTAL & 1776 & 341 & 216 & 2333 & 4.1 & 3.9 & 2.5 & 3.8 \\
\hline \multicolumn{9}{|l|}{ Defects of genitourinary system } \\
\hline Defects of female genitals & 63 & 5 & 8 & 76 & 0.1 & 0.1 & 0.1 & 0.1 \\
\hline Undescended testis & 455 & 71 & 21 & 547 & 1.1 & 0.8 & 0.2 & 0.9 \\
\hline Hypospadias & 991 & 202 & 165 & 1358 & 2.3 & 2.3 & 1.9 & 2.2 \\
\hline Epispadias & 27 & 2 & 3 & 32 & 0.1 & 0.0 & 0.0 & 0.1 \\
\hline Chordee & 194 & 24 & 11 & 229 & 0.4 & 0.3 & 0.1 & 0.4 \\
\hline Indeterminate sex-ambiguous genitalia & a 70 & 9 & 10 & 89 & 0.2 & 0.1 & 0.1 & 0.1 \\
\hline Renal agenesis-dysgenesis & 169 & 38 & 29 & 236 & 0.4 & 0.4 & 0.3 & 0.4 \\
\hline $\begin{array}{l}\text { Obstructive defects of renal pelvis } \\
\text { and ureter }\end{array}$ & 828 & 165 & 61 & 1054 & 19 & 19 & 07 & 17 \\
\hline Other genitourinary system defects & $\begin{array}{l}0<0 \\
742\end{array}$ & 109 & 59 & $\begin{array}{r}1054 \\
910\end{array}$ & $\begin{array}{l}1.9 \\
1.7\end{array}$ & $\begin{array}{l}1.9 \\
1.3\end{array}$ & 0.7 & $\begin{array}{l}1.7 \\
1.5\end{array}$ \\
\hline TOTAL & 3539 & 625 & 367 & 4531 & 8.2 & 7.2 & 4.2 & 7.5 \\
\hline \multicolumn{9}{|l|}{ Defects of musculoskeletal system } \\
\hline Congenital dislocation of the hips & 837 & 122 & 86 & 1045 & 1.9 & 1.4 & 1.0 & 1.7 \\
\hline Talipes equinovarus & 290 & 55 & 37 & 382 & 0.7 & 0.6 & 0.4 & 0.6 \\
\hline Polydactyly & 475 & 95 & 84 & 654 & 1.1 & 1.1 & 1.0 & 1.1 \\
\hline Syndactyly & 154 & 23 & 15 & 192 & 0.4 & 0.3 & 0.2 & 0.3 \\
\hline Reduction deformities of limbs & 327 & 59 & 50 & 436 & 0.8 & 0.7 & 0.6 & 0.7 \\
\hline Craniosynostosis & 448 & 69 & 7 & 524 & 1.0 & 0.8 & 0.1 & 0.9 \\
\hline Diaphragmatic hernia & 126 & 38 & 18 & 182 & 0.3 & 0.4 & 0.2 & 0.3 \\
\hline Exomphalos & 73 & 14 & 13 & 100 & 0.2 & 0.2 & 0.1 & 0.2 \\
\hline Gastroschisis & 84 & 18 & 19 & 121 & 0.2 & 0.2 & 0.2 & 0.2 \\
\hline Other musculoskeletal defects & 1248 & 193 & 152 & 1593 & 2.9 & 2.2 & 1.7 & 2.6 \\
\hline TOTAL & 4062 & 686 & 481 & 5229 & 9.4 & 7.9 & 5.5 & 8.6 \\
\hline Defects of integumentary system & 366 & 60 & 52 & 478 & 0.8 & 0.7 & 0.6 & 0.8 \\
\hline Cystic hygroma & 49 & 8 & 4 & 61 & 0.1 & 0.1 & 0.0 & 0.1 \\
\hline \multicolumn{9}{|l|}{ Chromosomal defects } \\
\hline Trisomy 21 & 554 & 93 & 90 & 737 & 1.3 & 1.1 & 1.0 & 1.2 \\
\hline Trisomy 13 & 24 & 8 & 10 & 42 & 0.1 & 0.1 & 0.1 & 0.1 \\
\hline Trisomy 18 & 86 & 22 & 14 & 122 & 0.2 & 0.3 & 0.2 & 0.2 \\
\hline Turner syndrome & 48 & 11 & 8 & 67 & 0.1 & 0.1 & 0.1 & 0.1 \\
\hline Other chromosomal defects & 219 & 61 & 28 & 308 & 0.5 & 0.7 & 0.3 & 0.5 \\
\hline TOTAL & 931 & 195 & 150 & 1276 & 2.2 & 2.3 & 1.7 & 2.1 \\
\hline Situs inversus & 20 & 4 & 3 & 27 & 0.0 & 0.0 & 0.0 & 0.0 \\
\hline Congenital malformation syndromes & 201 & 32 & 32 & 265 & 0.5 & 0.4 & 0.4 & 0.4 \\
\hline Congenital rubella syndrome & 5 & 0 & 0 & 5 & 0.0 & 0.0 & 0.0 & 0.0 \\
\hline Congenital cytomegalovirus infection & 12 & 1 & 0 & 13 & 0.0 & 0.0 & 0.0 & 0.0 \\
\hline Congenital toxoplasmosis & 1 & 1 & 0 & 2 & 0.0 & 0.0 & 0.0 & 0.0 \\
\hline Non-immune hydrops foetalis & 112 & 28 & 14 & 154 & 0.3 & 0.3 & 0.2 & 0.3 \\
\hline Other and unspecified birth defects & 590 & 115 & 19 & 724 & 1.4 & 1.3 & 0.2 & 1.2 \\
\hline TOTAL & 18540 & 3379 & 2017 & 23936 & 42.9 & 39.1 & 23.1 & 39.5 \\
\hline
\end{tabular}

Source: NSW Birth Defects Register. Epidemiology and Surveillance Branch, NSW Department of Health.

\# $\quad$ For 1994-1999, cases reported during pregnancy and up to one year of age are included. For 2000, cases reported during pregnancy or at birth are reported. 


\subsubsection{INFANT CHARACTERISTICS}

In the period 1994-2000, a single defect was reported in 62.9 per cent of infants, two defects in 17.5 per cent, three defects in 8.0 per cent, and four or more defects in 11.6 per cent of cases.

The sex was male in 59.0 per cent of infants, female in 40.3 per cent, indeterminate in 0.4 per cent of infants, and was not stated for 0.4 per cent.

Birth defects were more common in preterm and post-term infants than infants born at term (Table 98). Birth defects were also more common in infants born of a multiple pregnancy than a singleton pregnancy: in 1994-2000, 2.0 per cent of singleton babies, 2.8 per cent of twins and 3.3 per cent of triplets were born with a birth defect.

Ten per cent of infants born with birth defects died in the perinatal period, with stillbirths contributing just over half the perinatal deaths (Table 99). These figures comprise all birth defect cases, including those where the cause of death may not be directly related to the birth defect(s). By comparison, the perinatal mortality rate among all births reported to the NSW Midwives Data Collection was 9.7 per 1,000 in 2000 (see Section 1.16).

\section{TABLE 98}

BIRTH DEFECT CASES BY GESTATIONAL AGE, NSW 1994-2000\#

\begin{tabular}{|c|c|c|c|c|c|c|c|c|c|}
\hline \multirow{3}{*}{$\begin{array}{l}\text { Gestational age } \\
\text { (weeks) }\end{array}$} & \multirow{2}{*}{\multicolumn{9}{|c|}{ 1994-2000 }} \\
\hline & & & & & & & & & \\
\hline & No. & $\%$ & No. & $\%$ & No. & $\%$ & No. & $\%$ & Rate/1,000 births \\
\hline $20-27$ & 426 & 4.4 & 106 & 5.8 & 83 & 7.9 & 615 & 4.9 & 160.2 \\
\hline $28-31$ & 285 & 2.9 & 50 & 2.7 & 30 & 2.8 & 365 & 2.9 & 86.5 \\
\hline $32-36$ & 1077 & 11.0 & 233 & 12.7 & 132 & 12.5 & 1442 & 11.4 & 43.5 \\
\hline $37-41$ & 7366 & 75.4 & 1365 & 74.3 & 790 & 74.8 & 9521 & 75.2 & 17.3 \\
\hline $42+$ & 261 & 2.7 & 41 & 2.2 & 20 & 1.9 & 322 & 2.5 & 21.0 \\
\hline Not stated & 351 & 3.6 & 41 & 2.2 & 1 & 0.1 & 393 & 3.1 & - \\
\hline TOTAL & 9766 & 100.0 & 1836 & 100.0 & 1056 & 100.0 & 12658 & 100.0 & 20.9 \\
\hline
\end{tabular}

Source: NSW Birth Defects Register. Epidemiology and Surveillance Branch, NSW Department of Health.

\# For 1994-1999, cases reported during pregnancy and up to one year of age are included. For 2000, cases reported during pregnancy or at birth are reported.

\section{TABLE 99}

BIRTH DEFECT CASES BY PREGNANCY OUTCOME, NSW 1994-2000"

\begin{tabular}{|c|c|c|c|c|c|c|c|c|}
\hline \multirow{3}{*}{ Pregnancy outcome } & \multicolumn{8}{|c|}{ Year } \\
\hline & \multicolumn{2}{|c|}{ 1994-1998 } & \multicolumn{2}{|c|}{1999} & \multicolumn{2}{|c|}{2000} & \multicolumn{2}{|c|}{ 1994-2000 } \\
\hline & No. & $\%$ & No. & $\%$ & No. & $\%$ & No. & $\%$ \\
\hline Stillbirth & 484 & 5.0 & 103 & 5.6 & 99 & 9.4 & 686 & 5.4 \\
\hline Liveborn-neonatal death & 430 & 4.4 & 94 & 5.1 & 52 & 4.9 & 576 & 4.6 \\
\hline Liveborn-postneonatal death & 86 & 0.9 & 16 & 0.9 & 7 & 0.7 & 109 & 0.9 \\
\hline Liveborn surviving & 8766 & 89.8 & 1623 & 88.4 & 898 & 85.0 & 11287 & 89.2 \\
\hline TOTAL & 9766 & 100.0 & 1836 & 100.0 & 1056 & 100.0 & 12658 & 100.0 \\
\hline
\end{tabular}

Source: NSW Birth Defects Register. Epidemiology and Surveillance Branch, NSW Department of Health.

\# For 1994-1999, cases reported during pregnancy and up to one year of age are included. For 2000, cases reported during pregnancy or at birth are reported. 


\subsubsection{MATERNAL CHARACTERISTICS}

After 30 years of age, the incidence of birth defects increased with increasing maternal age (Table 100). While the rate of birth defects is higher in older women, the majority of births occur in younger women: in 1994-2000, 77.3 per cent of babies with birth defects were born to women aged less than 35 years.

In 1994-2000, 140 babies of Aboriginal or Torres Strait Islander mothers were reported to have birth defects. The rate of birth defects among these babies was 16.3 per 1,000 compared with 21.0 per 1,000 for non-Aboriginal mothers.

\section{TABLE 100}

BIRTH DEFECT CASES BY MATERNAL AGE, NSW 1994-2000\#

\begin{tabular}{|c|c|c|c|c|c|c|c|c|c|}
\hline \multirow[t]{2}{*}{$\begin{array}{l}\text { Maternal age } \\
\text { (years) }\end{array}$} & \multicolumn{9}{|c|}{ 1994-1998 } \\
\hline & No. & $\%$ & No. & $\%$ & No. & $\%$ & No. & $\%$ & Rate/1,000 births \\
\hline Under 20 & 498 & 5.1 & 87 & 4.7 & 59 & 5.6 & 644 & 5.1 & 22.0 \\
\hline $20-24$ & 1586 & 16.2 & 267 & 14.5 & 156 & 14.8 & 2009 & 15.9 & 19.1 \\
\hline 25-29 & 2795 & 28.6 & 574 & 31.3 & 310 & 29.4 & 3679 & 29.1 & 18.8 \\
\hline $30-34$ & 2674 & 27.4 & 479 & 26.1 & 304 & 28.8 & 3457 & 27.3 & 19.0 \\
\hline 35-39 & 1264 & 12.9 & 276 & 15.0 & 174 & 16.5 & 1714 & 13.5 & 21.3 \\
\hline $40-44$ & 281 & 2.9 & 75 & 4.1 & 50 & 4.7 & 406 & 3.2 & 30.0 \\
\hline $45+$ & 19 & 0.2 & 3 & 0.2 & 3 & 0.3 & 25 & 0.2 & 47.8 \\
\hline Not stated & 649 & 6.6 & 75 & 4.1 & 0 & 0.0 & 724 & 5.7 & - \\
\hline TOTAL & 9766 & 100.0 & 1836 & 100.0 & 1056 & 100.0 & 12658 & 100.0 & 20.9 \\
\hline
\end{tabular}

Source: NSW Birth Defects Register. Epidemiology and Surveillance Branch, NSW Department of Health.

\# $\quad$ For 1994-1999, cases reported during pregnancy and up to one year of age are included. For 2000, cases reported during pregnancy or at birth are reported.

\subsection{BIRTH DEFECTS AMONG TERMINATIONS OF PREGNANCY, SPONTANEOUS ABORTIONS AND UNKNOWN OUTCOMES OF PREGNANCY}

In the period 1994-1998, about 150 terminations of pregnancy per year were reported to the NSW Birth Defects Register (Table 101). Following the introduction of a requirement to notify birth defects under the NSW Public Health Act 1991 from 1 January 1998, the number of terminations reported rose to 250 in 1998 and 308 in 1999.

Of the total 1,222 terminations of pregnancy reported in 1994-2000, 706 (57.8 per cent) were associated with a chromosomal abnormality, the most common of which was Down syndrome, and 276 (22.6 per cent) were associated with a neural tube defect (Table 102). In 1994-2000, 55.0 per cent of terminations were carried out in women aged less than 35 years (Table 103).

For spontaneous abortions, cytogenetic analysis is only carried out in cases of habitual abortion and the numbers presented therefore underestimate the number of spontaneous abortions which occur due to birth defects. Descriptions of some diagnostic terms used here are included in Appendix 1.

\section{TABLE 101}

PREGNANCIES WITH FETUSES AFFECTED BY BIRTH DEFECTS AND RESULTING IN SPONTANEOUS ABORTION, TERMINATION OF PREGNANCY OR UNKNOWN OUTCOME, NSW 1994-2000

\begin{tabular}{|c|c|c|c|c|}
\hline \multirow{3}{*}{ Pregnancy outcome } & \multicolumn{4}{|c|}{ Year } \\
\hline & \multirow{2}{*}{$\begin{array}{c}\text { 1994-1998 } \\
\text { No. }\end{array}$} & \multirow{2}{*}{$\begin{array}{l}1999 \\
\text { No. }\end{array}$} & \multicolumn{2}{|c|}{ 20001994-2000 } \\
\hline & & & No. & No. \\
\hline Spontaneous abortion & 309 & 118 & 108 & 535 \\
\hline $\begin{array}{l}\text { Termination of } \\
\text { pregnancy less than }\end{array}$ & & & & \\
\hline 20 weeks gestation & 774 & 308 & 140 & 1222 \\
\hline Unknown outcome & 623 & 17 & 0 & 640 \\
\hline TOTAL & 1706 & 443 & 248 & 2397 \\
\hline
\end{tabular}

Source: NSW Birth Defects Register. Epidemiology and Surveillance Branch, NSW Department of Health. 


\section{TABLE 102}

BIRTH DEFECTS AMONG SPONTANEOUS ABORTIONS, TERMINATIONS OF PREGNANCY AND UNKNOWN OUTCOME OF PREGNANCY BY DIAGNOSTIC CATEGORY, NSW 1994-2000

\begin{tabular}{|c|c|c|c|c|c|c|c|c|c|c|c|}
\hline \multirow[t]{2}{*}{ Diagnostic category } & \multirow[t]{2}{*}{$\begin{array}{l}\text { Spont. } \\
\text { abortion } \\
\text { No. }\end{array}$} & \multirow[t]{2}{*}{$\begin{array}{l}\text { 1994-1998 } \\
\text { Termination } \\
\text { of pregnancy } \\
\text { less than } \\
20 \text { weeks } \\
\text { gestation } \\
\text { No. }\end{array}$} & \multirow[t]{2}{*}{$\begin{array}{l}\text { Unknown } \\
\text { outcome } \\
\text { No. }\end{array}$} & \multirow[t]{2}{*}{$\begin{array}{c}\text { Spont. } \\
\text { abortion } \\
\text { No. }\end{array}$} & \multirow[t]{2}{*}{$\begin{array}{c}\text { Year } \\
1999 \\
\text { Termination } \\
\text { of pregnancy } \\
\text { less than } \\
20 \text { weeks } \\
\text { gestation } \\
\text { No. }\end{array}$} & \multirow[t]{2}{*}{$\begin{array}{r}\text { Unknown } \\
\text { outcome } \\
\text { No. }\end{array}$} & \multirow[t]{2}{*}{$\begin{array}{l}\begin{array}{c}\text { Spont. } \\
\text { abortion }\end{array} \\
\text { No. }\end{array}$} & \multirow[t]{2}{*}{$\begin{array}{c}2000 \\
\text { Termination } \\
\text { of pregnancy } \\
\text { less than } \\
20 \text { weeks } \\
\text { gestation } \\
\text { No. }\end{array}$} & \multirow{2}{*}{\multicolumn{2}{|c|}{$\begin{array}{c}\text { 1994-2000 } \\
\text { Spont. Termination } \\
\text { abortion of pregnancy } \\
\text { less than } \\
20 \text { weeks } \\
\text { gestation } \\
\text { No. No. }\end{array}$}} & \multirow[t]{2}{*}{$\begin{array}{r}\text { OUnknown } \\
\text { outcome } \\
\text { No. }\end{array}$} \\
\hline & & & & & & & & & & & \\
\hline \multicolumn{12}{|l|}{ Defects of nervous system } \\
\hline $\begin{array}{l}\text { Neural tube defects } \\
\text { Other nervous system }\end{array}$ & 14 & 192 & 7 & 0 & 48 & 1 & 1 & 36 & 15 & 276 & 8 \\
\hline defects & 6 & 82 & 11 & 0 & 33 & 1 & 2 & 19 & 8 & 134 & 12 \\
\hline TOTAL & 20 & 274 & 18 & 0 & 81 & 2 & 3 & 55 & 23 & 410 & 20 \\
\hline $\begin{array}{l}\text { Defects of eye } \\
\text { Defects of ear, face and }\end{array}$ & 0 & 2 & 1 & 0 & 1 & 0 & 0 & 0 & 0 & 3 & 1 \\
\hline neck & 0 & 7 & 1 & 0 & 4 & 0 & 0 & 2 & 0 & 13 & 1 \\
\hline system & 4 & 105 & 44 & 0 & 51 & 3 & 4 & 19 & 8 & 175 & 47 \\
\hline system & 0 & 18 & 3 & 0 & 12 & 0 & 0 & 0 & 0 & 30 & 3 \\
\hline $\begin{array}{l}\text { gastrointestinal system } \\
\text { Defects of genitourinary }\end{array}$ & 3 & 59 & 13 & 0 & 35 & 0 & 2 & 17 & 5 & 111 & 13 \\
\hline $\begin{array}{l}\text { system } \\
\text { Defects of musculoskeletal }\end{array}$ & 7 & 131 & 22 & 4 & 42 & 0 & 2 & 38 & 13 & 211 & 22 \\
\hline $\begin{array}{l}\text { system } \\
\text { Defects of the integumentar }\end{array}$ & 19 & 270 & 33 & 4 & 95 & 1 & 6 & 51 & 29 & 416 & 34 \\
\hline system & 1 & 1 & 1 & 0 & 1 & 0 & 0 & 0 & 1 & 2 & 1 \\
\hline $\begin{array}{l}\text { Cystic hygroma } \\
\text { Chromosomal defects }\end{array}$ & 8 & 71 & 29 & 4 & 20 & 0 & 1 & 11 & 13 & 102 & 29 \\
\hline Trisomy 21 & 29 & 173 & 231 & 10 & 107 & 2 & 7 & 44 & 46 & 324 & 233 \\
\hline Trisomy 13 & 11 & 27 & 32 & 5 & 15 & 1 & 8 & 8 & 24 & 50 & 33 \\
\hline Trisomy 18 & 18 & 76 & 79 & 7 & 41 & 4 & 4 & 9 & 29 & 126 & 83 \\
\hline Turner syndrome & 32 & 30 & 39 & 10 & 15 & 0 & 11 & 9 & 53 & 54 & 39 \\
\hline $\begin{array}{l}\text { Other chromosomal } \\
\text { defects }\end{array}$ & 199 & 98 & 163 & 80 & 41 & 6 & 76 & 13 & 355 & 152 & 169 \\
\hline TOTAL & 289 & 404 & 544 & 112 & 219 & 13 & 106 & 83 & 507 & 706 & 557 \\
\hline $\begin{array}{l}\text { Situs inversus } \\
\text { Congenital malformation }\end{array}$ & 0 & 2 & 0 & 0 & 0 & 0 & 0 & 1 & 0 & 3 & 0 \\
\hline $\begin{array}{l}\text { syndromes } \\
\text { Non-immune hydrops }\end{array}$ & 0 & 20 & 2 & 0 & 3 & 0 & 0 & 2 & 0 & 25 & 2 \\
\hline $\begin{array}{l}\text { foetalis } \\
\text { Other and unspecified }\end{array}$ & 3 & 28 & 9 & 2 & 14 & 2 & 1 & 11 & 6 & 53 & 11 \\
\hline birth defects & 1 & 22 & 23 & 0 & 11 & 1 & 2 & 1 & 3 & 34 & 24 \\
\hline TOTAL & 355 & 1414 & 743 & 126 & 589 & 22 & 127 & 291 & 608 & 2294 & 765 \\
\hline
\end{tabular}

Source: NSW Birth Defects Register. Epidemiology and Surveillance Branch, NSW Department of Health.

\section{TABLE 103}

TRENDS IN REPORTED TERMINATIONS OF PREGNANCY ASSOCIATED WITH BIRTH DEFECTS BY MATERNAL AGE, 1994-2000

\begin{tabular}{|c|c|c|c|c|c|c|c|c|c|c|c|c|c|c|c|c|c|c|}
\hline \multirow[t]{3}{*}{ Year } & \multicolumn{18}{|c|}{ Maternal age (years) } \\
\hline & \multicolumn{2}{|c|}{ 15-19 } & \multicolumn{2}{|c|}{$20-24$} & \multicolumn{2}{|c|}{$25-29$} & \multicolumn{2}{|c|}{$30-34$} & \multicolumn{2}{|c|}{$35-39$} & \multicolumn{2}{|c|}{$40-44$} & \multicolumn{2}{|c|}{$45+$} & \multicolumn{2}{|c|}{ Not stated } & \multicolumn{2}{|c|}{ TOTAL } \\
\hline & No & $\%$ & No & $\%$ & No & $\%$ & No & $\%$ & No & $\%$ & No & $\%$ & No & $\%$ & No & $\%$ & No & $\%$ \\
\hline 1994 & 5 & 3.6 & 26 & 18.8 & 20 & 14.5 & 23 & 16.7 & 29 & 21.0 & 17 & 12.3 & 2 & 1.4 & 16 & 11.6 & 138 & 100.0 \\
\hline 1995 & 6 & 3.9 & 19 & 12.3 & 31 & 20.0 & 38 & 24.5 & 33 & 21.3 & 23 & 14.8 & 2 & 1.3 & 3 & 1.9 & 155 & 100.0 \\
\hline 1996 & 3 & 2.8 & 16 & 15.1 & 22 & 20.8 & 24 & 22.6 & 24 & 22.6 & 11 & 10.4 & 0 & 0.0 & 6 & 5.7 & 106 & 100.0 \\
\hline 1997 & 3 & 2.4 & 13 & 10.4 & 33 & 26.4 & 32 & 25.6 & 25 & 20.0 & 13 & 10.4 & 1 & 0.8 & 5 & 4.0 & 125 & 100.0 \\
\hline 1998 & 3 & 1.2 & 19 & 7.6 & 55 & 22.0 & 45 & 18.0 & 63 & 25.2 & 51 & 20.4 & 4 & 1.6 & 10 & 4.0 & 250 & 100.0 \\
\hline 1999 & 6 & 1.9 & 19 & 6.2 & 56 & 18.2 & 72 & 23.4 & 90 & 29.2 & 42 & 13.6 & 5 & 1.6 & 18 & 5.8 & 308 & 100.0 \\
\hline 2000 & 2 & 1.4 & 11 & 7.9 & 30 & 21.4 & 40 & 28.6 & 32 & 22.9 & 15 & 10.7 & 1 & 0.7 & 9 & 6.4 & 140 & 100.0 \\
\hline 1994-2000 & 28 & 2.3 & 123 & 10.1 & 247 & 20.2 & 274 & 22.4 & 296 & 24.2 & 172 & 14.1 & 15 & 1.2 & 67 & 5.5 & 1222 & 100.0 \\
\hline
\end{tabular}

Source: NSW Birth Defects Register. Epidemiology and Surveillance Branch, NSW Department of Health. 


\subsection{TRENDS IN SELECTED BIRTH DEFECTS}

Trends in a selection of common birth defects are shown in Figures 13 to 20. For 1994-1999, malformations reported up to one year of age are included and for 2000 malformations reported during pregnancy or at birth are included.

The reported number of infants born with neural tube was 46 in 1994 and 41 in 1999, and 31 have been reported for 2000 to date. The number of reported terminations of pregnancy was 40 in 1994, 44 in 1999 and 32 in 2000.

Over the period 1994-1999, the number of cases of isolated cleft palate ranged from 59 in 2000 to 91 in 1995 and for total cleft lip (including cases of cleft lip and cleft palate) from 70 in 2000 to 101 in 1995 (Figures 14 and 15). Termination of pregnancy was usually associated with other defects such as neural tube defects, chromosomal abnormalities or multiple abnormalities in addition to the cleft lip and/or cleft palate.

The number of reported cases of hypospadias varied from 165 in 2000 to 224 in 1993 (Figure 16), and cases of limb reduction defects varied from 44 in 1996 to 61 in 1997 (Figure 17).
The number of reported terminations of pregnancy for chromosomal abnormalities, including Down syndrome, increased following the introduction of a requirement to notify birth defects under the NSW Public Health Act 1991 from 1 January 1998 (Figures 18 and 19). The number of infants born with chromosomal defects was 185 in 1994 to 194 in 1999, and the number of reported terminations of pregnancy associated with chromosomal defects rose from 69 in 1994 to 219 in 1999. The number of infants born with Down syndrome was 104 in 1999 and 93 in 1999, while the number of terminations of pregnancy associated with Down syndrome rose from 26 in 1994 to 107 in 1999.

There was a trend towards improved notification of cases of renal agenesis and dysgenesis, which peaked in 1998. The increased reporting is due partly to the introduction of notification requirements in 1998, but also to improved diagnosis of less severe forms of renal dysgenesis in infants (Figure 20).

\section{FIGURE 13}

NEURAL TUBE DEFECTS: CASES BY YEAR AND PREGNANCY OUTCOME, NSW 1994-2000\#

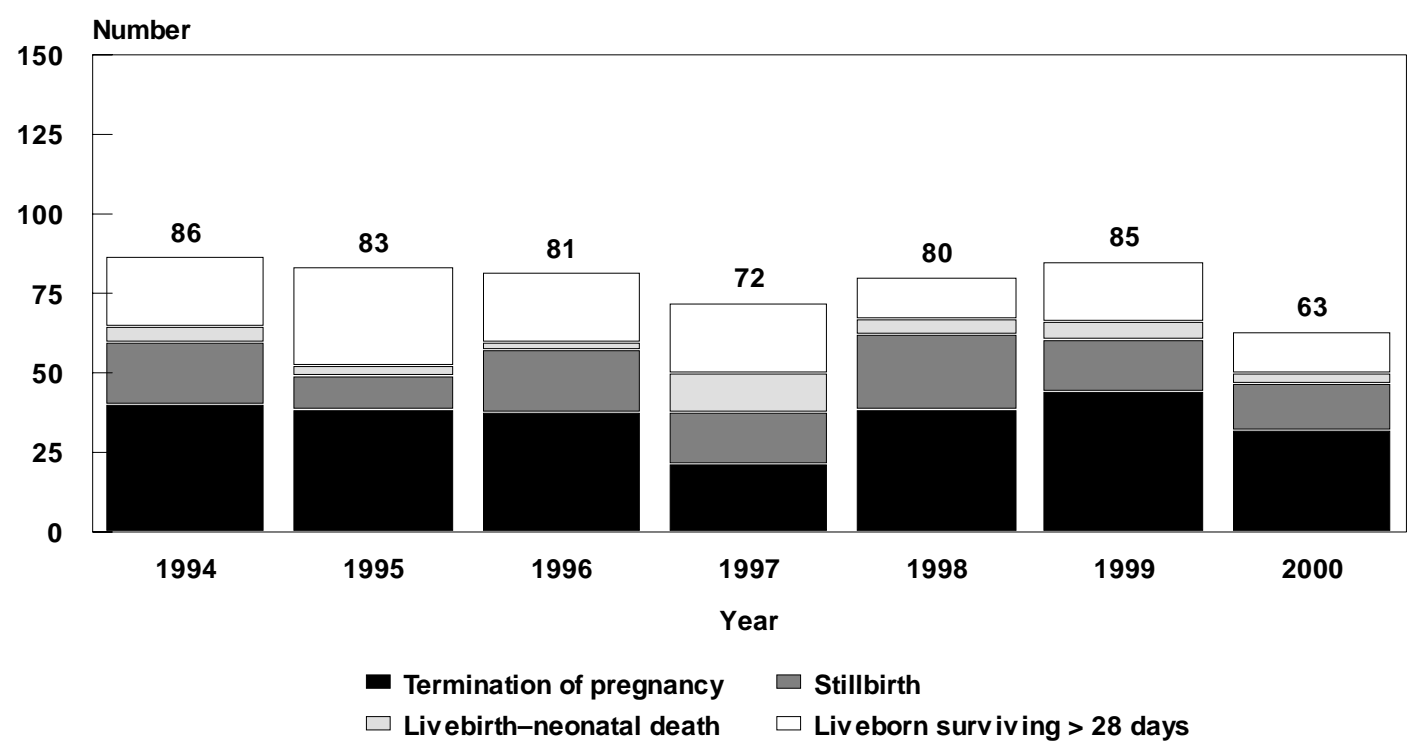

Source: NSW Birth Defects Register. Epidemiology and Surveillance Branch, NSW Department of Health.

\# $\quad$ For 1994-1999, cases reported during pregnancy and up to one year of age are included. For 2000, cases reported during pregnancy or at birth are reported. 


\section{FIGURE 14}

CLEFT PALATE: CASES BY YEAR AND PREGNANCY OUTCOME, NSW 1994-2000\#

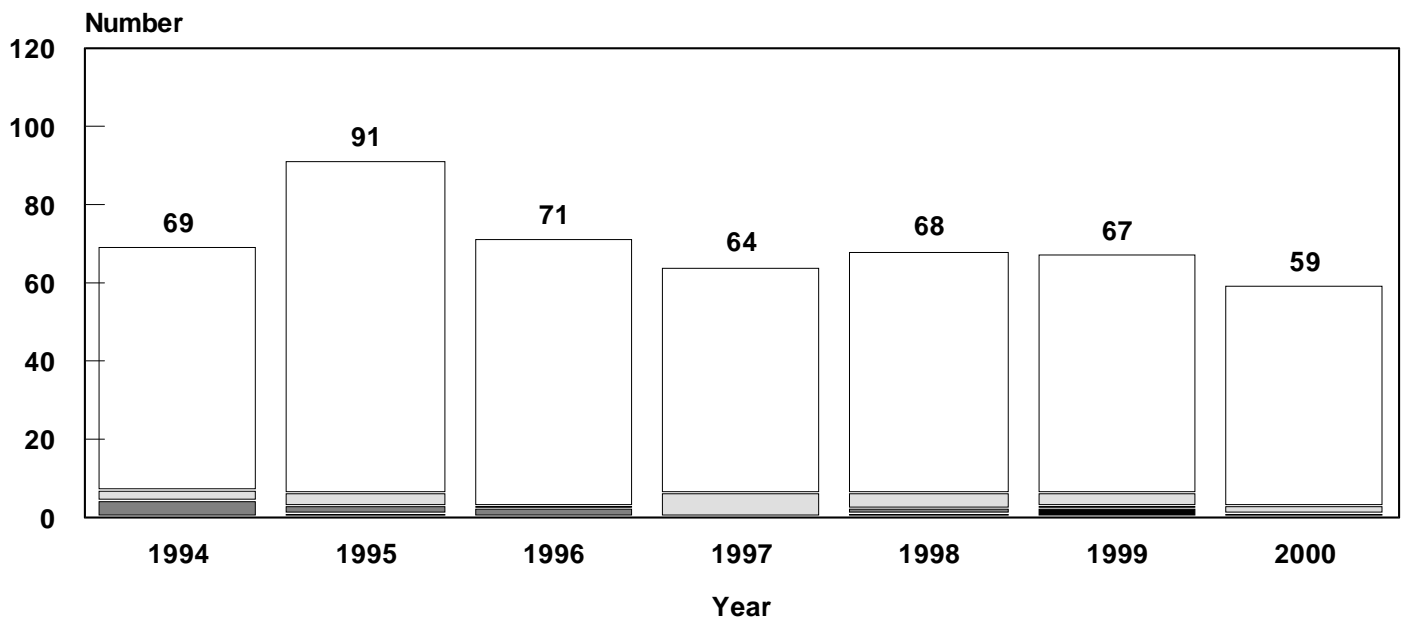

- Termination of pregnancy

$\square$ Stillbirth

$\square$ Liv ebirth-neonatal death $\square$ Liv eborn surviving > 28 days

Source: NSW Birth Defects Register. Epidemiology and Surveillance Branch, NSW Department of Health.

\# $\quad$ For 1994-1999, cases reported during pregnancy and up to one year of age are included. For 2000, cases reported during pregnancy or at birth are reported.

\section{FIGURE 15}

TOTAL CLEFT LIP: CASES BY YEAR AND PREGNANCY OUTCOME, NSW 1994-2000\#

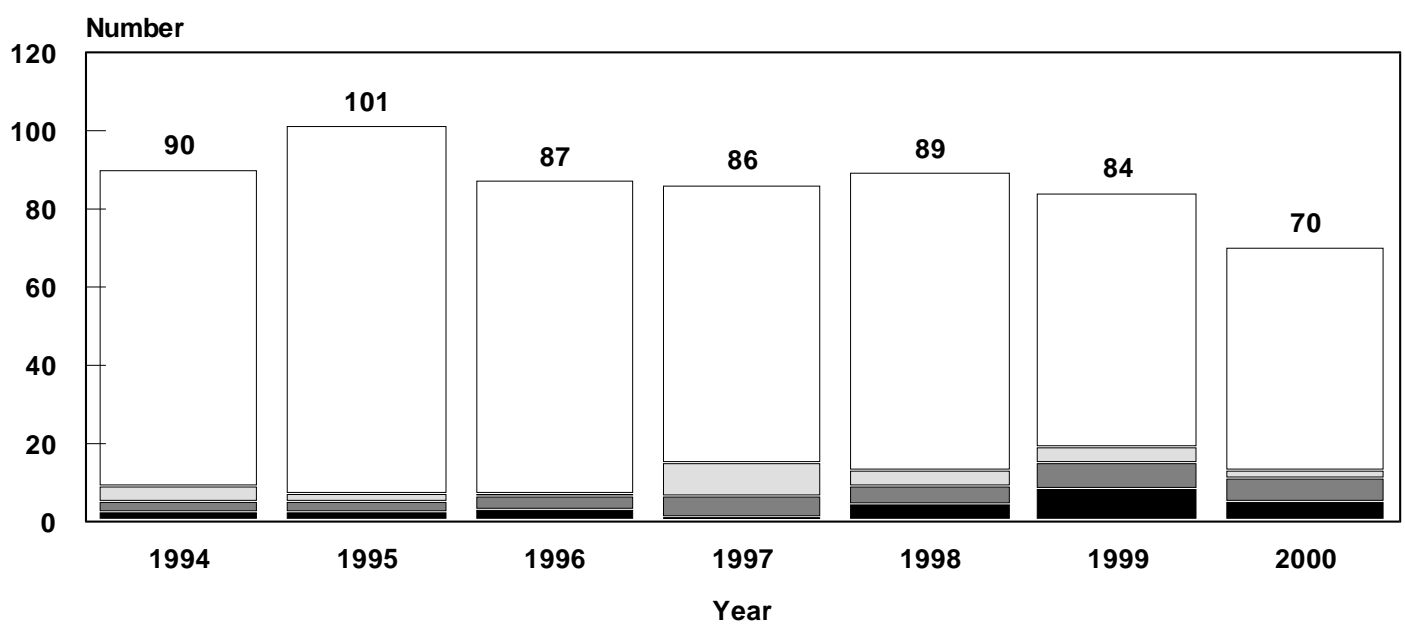

\footnotetext{
- Termination of pregnancy $\square$ Stillbirth

$\square$ Livebirth-neonatal death $\square$ Liv eborn surviving > 28 days
}

Source: NSW Birth Defects Register. Epidemiology and Surveillance Branch, NSW Department of Health.

\# $\quad$ For 1994-1999, cases reported during pregnancy and up to one year of age are included. For 2000, cases reported during pregnancy or at birth are reported. 


\section{FIGURE16}

HYPOSPADIAS: CASES BY YEAR AND PREGNANCY OUTCOME, NSW 1994-2000\#

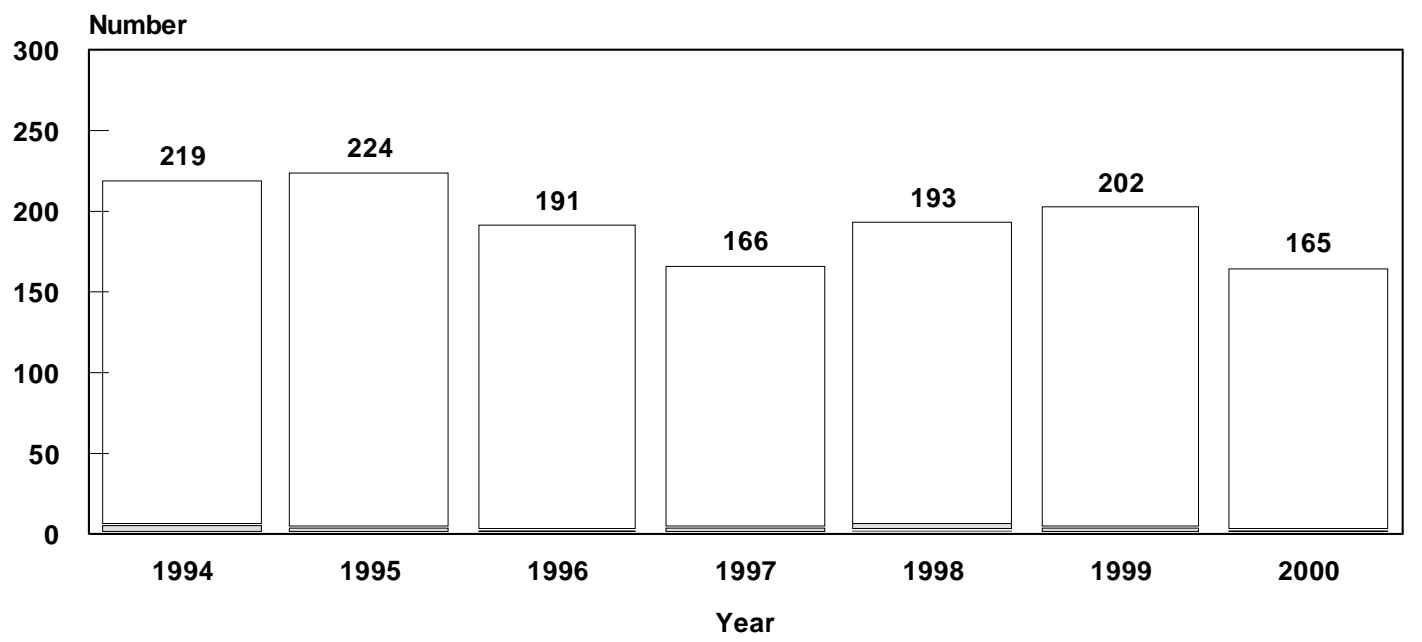

Termination of pregnancy

$\square$ Stillbirth

$\square$ Liv ebirth-neonatal death

$\square$ Liv eborn surviving $>28$ days

Source: NSW Birth Defects Register. Epidemiology and Surveillance Branch, NSW Department of Health.

\# For 1994-1999, cases reported during pregnancy and up to one year of age are included. For 2000, cases reported during pregnancy or at birth are reported.

\section{FIGURE 17}

LIMB REDUCTION DEFECTS: CASES BY YEAR AND PREGNANCY OUTCOME, NSW 1994-2000\#

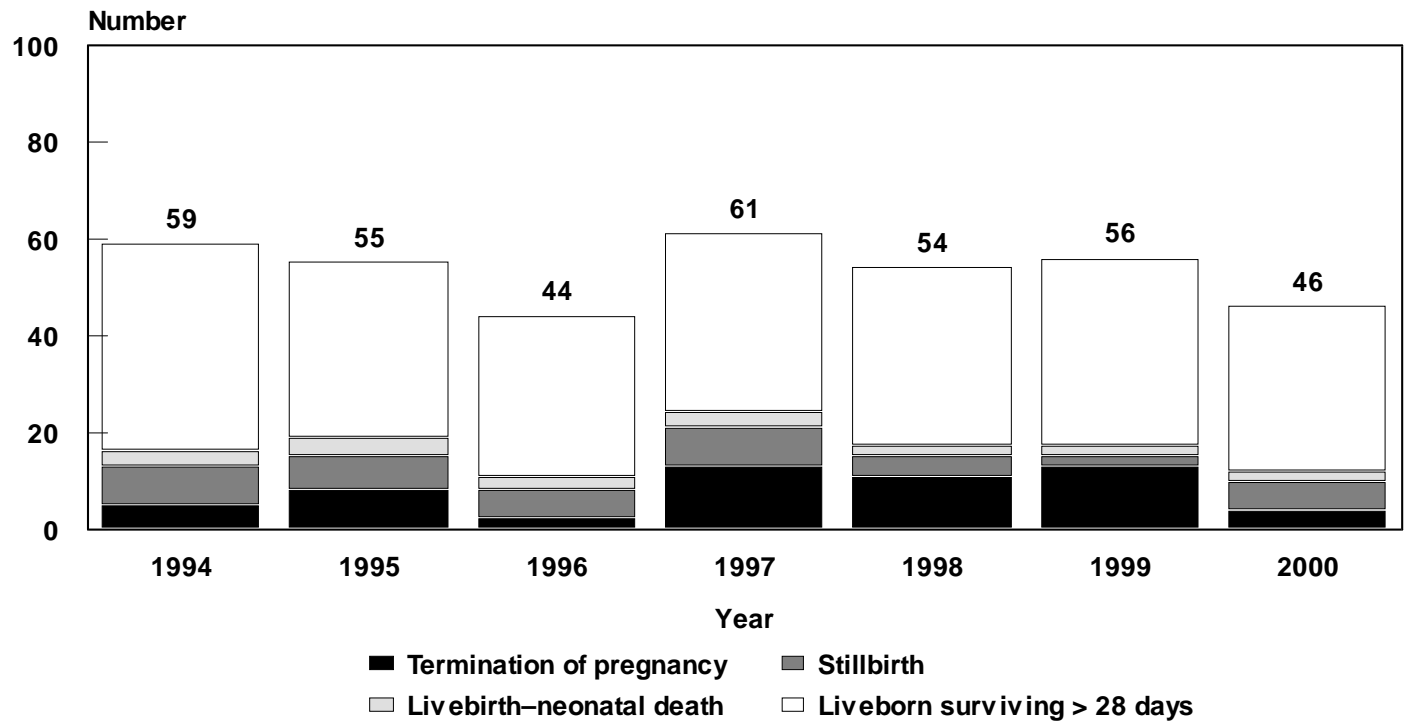

Source: NSW Birth Defects Register. Epidemiology and Surveillance Branch, NSW Department of Health

\# $\quad$ For 1994-1999, cases reported during pregnancy and up to one year of age are included. For 2000, cases reported during pregnancy or at birth are reported. 
FIGURE 18

CHROMOSOMAL ABNORMALITIES: CASES BY YEAR AND PREGNANCY OUTCOME, NSW 1994-2000\#

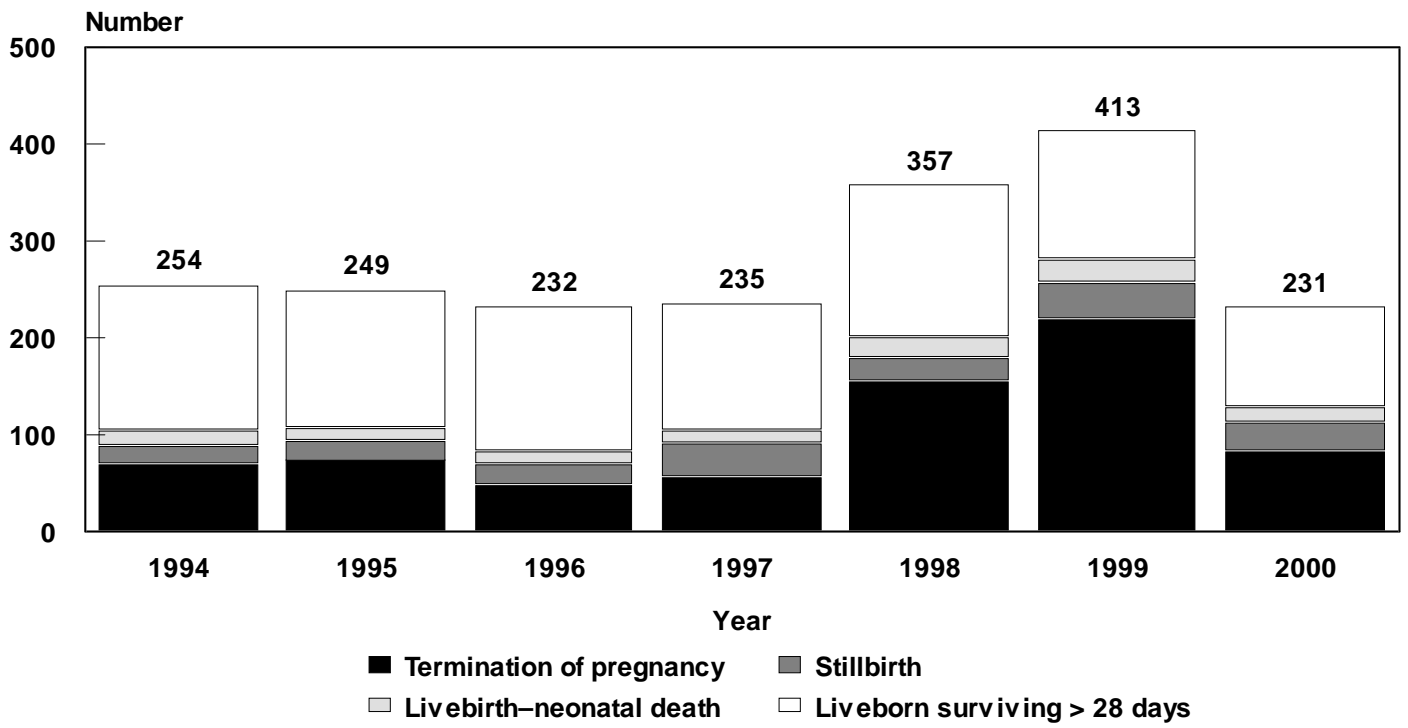

Source: NSW Birth Defects Register. Epidemiology and Surveillance Branch, NSW Department of Health.

\# $\quad$ From 1 January 1998 birth defects are notifiable under the NSW Public Health Act 1991. The increase in reported terminations of pregnancy in 1998 follows the introduction of this notification requirement. For 1994-1999, cases reported during pregnancy and up to one year of age are included. For 2000, cases reported during pregnancy or at birth are reported.

\section{FIGURE 19}

DOWN SYNDROME: CASES BY YEAR AND PREGNANCY OUTCOME, NSW 1994-2000\#

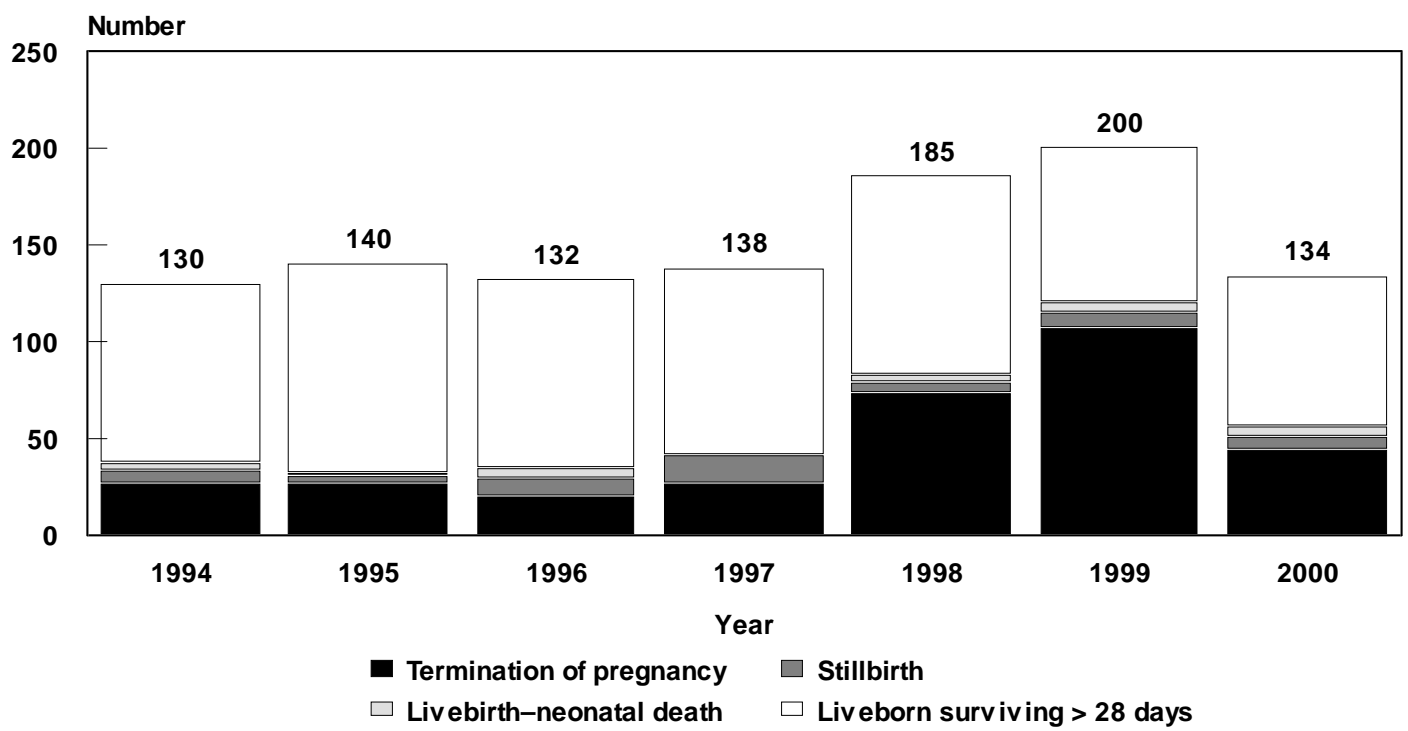

Source: NSW Birth Defects Register. Epidemiology and Surveillance Branch, NSW Department of Health.

\# $\quad$ From 1 January 1998 birth defects are notifiable under the NSW Public Health Act 1991. The increase in reported terminations of pregnancy in 1998 follows the introduction of this notification requirement. For 1994-1999, cases reported during pregnancy and up to one year of age are included. For 2000, cases reported during pregnancy or at birth are reported. 


\section{FIGURE 20}

RENAL AGENESIS AND DYSGENESIS: CASES BY YEAR AND PREGNANCY OUTCOME, NSW 1994-2000\#

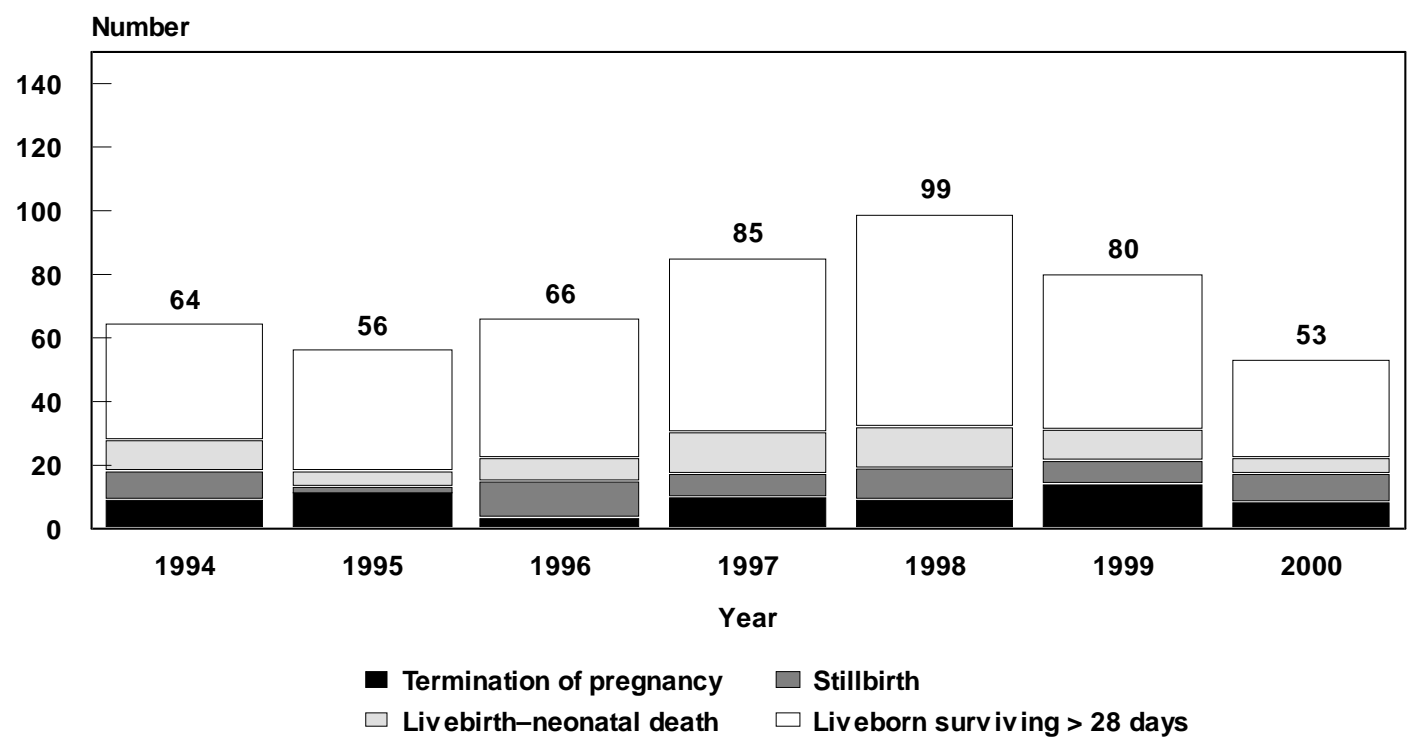

Source: NSW Birth Defects Register. Epidemiology and Surveillance Branch, NSW Department of Health.

\# Includes cystic renal disease and excludes obstructive defects of the renal pelvis, abnormally shaped kidney, double/triple kidney, ectopic kidney and enlarged kidney without dysplasia. For 1994-1999, cases reported during pregnancy and up to one year of age are included. For 2000, cases reported during pregnancy or at birth are reported. 


\subsection{BIRTH DEFECTS BY NSW HEALTH AREAS}

Crude rates of reported birth defects for NSW Health Areas and rates standardised for maternal age are shown in Table 104. For 1994-1999, birth defects detected up to one year of age are reported and for 2000 birth defects detected during pregnancy or at birth are reported. The denominator population includes live births and stillbirths among NSW residents as reported to the MDC. The rate of birth defects increases with increasing maternal age (Table 100). In order to allow direct comparison of geographic areas, rates have been standardised to the maternal age distribution of births in NSW in 1991.

Information shown in these tables reflects the reporting practices of the various areas. From 1 January 1998 doctors, hospitals and laboratories are required to notify birth defects detected during pregnancy, at birth or up to one year of life under NSW Public Health Act 1991. Thus higher rates of reported birth defects may be expected from 1998 onwards compared to previous years. In interpreting birth defect rates among NSW areas it should also be noted that infants with birth defects who are born to mothers resident in areas close to interstate borders may be transferred interstate for care and therefore may not be reported to the BDR.
Over the period 1994-2000 standardised rates of reported birth defects were lowest in the Central Sydney Health Area and highest in the Hunter Health Area. Review of cases showed slightly increased reported rates of several birth defects in the Hunter Area compared to NSW as a whole. Higher rates of neural tube defects and chromosomal abnormalities were reported in the Hunter Area, which is related to the relatively higher rates of notification of terminations of pregnancy for these conditions from the Hunter Area. In addition, higher rates of unstable hips (but not dislocated hips), hypospadias, and ventricular septal defect. The range of these defects suggests that enumeration of birth defects, including less severe conditions is better in the Hunter Health Area compared with NSW as a whole.

Birth defect rates may vary markedly from year to year for some areas where the numbers of reported birth defects are small. For these areas, small variations in numbers of birth defect cases may result in a marked variation in the birth defect rate. The wide confidence intervals for some areas reflect this variability.

\section{TABLE 104}

BIRTH DEFECTS IN NSW HEALTH AREAS, 1994-2000\#

\begin{tabular}{|c|c|c|c|c|c|c|c|c|c|c|c|c|c|}
\hline \multirow[t]{2}{*}{ Health Area } & \multirow[b]{2}{*}{ No. } & \multirow{2}{*}{\multicolumn{2}{|c|}{ 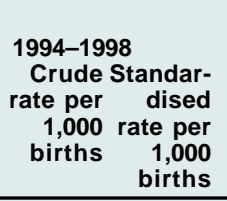 }} & \multirow[b]{2}{*}{ No. } & \multicolumn{5}{|c|}{ Year } & \multirow[b]{2}{*}{ No. } & \multirow{2}{*}{\multicolumn{2}{|c|}{ 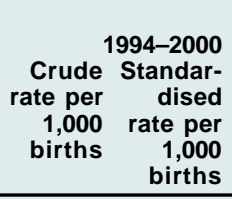 }} & \multirow[b]{2}{*}{$\begin{array}{r}99 \% \\
\text { confidence } \\
\text { intervals }\end{array}$} \\
\hline & & & & & $\begin{array}{r}1999 \\
\text { Crude } \\
\text { rate per } \\
1,000 \\
\text { births }\end{array}$ & $\begin{array}{r}\text { Standar- } \\
\text { dised } \\
\text { rate per } \\
1,000 \\
\text { births }\end{array}$ & No. & $\begin{array}{r}2000 \\
\text { Crude } \\
\text { rate per } \\
1,000 \\
\text { births }\end{array}$ & $\begin{array}{r}\text { Standar- } \\
\text { dised } \\
\text { rate per } \\
1,000 \\
\text { births }\end{array}$ & & & & \\
\hline Central Sydney & 739 & 21.7 & 20.3 & 145 & 21.6 & 18.9 & 97 & 14.0 & 14.1 & 981 & 20.6 & 19.3 & $17.5-21.1$ \\
\hline Northern Sydney & 1107 & 25.0 & 23.8 & 212 & 22.5 & 21.4 & 134 & 14.0 & 12.3 & 1453 & 22.9 & 22.0 & $20.0-24.1$ \\
\hline Western Sydney & 1247 & 23.8 & 22.9 & 269 & 24.8 & 23.8 & 142 & 13.0 & 13.2 & 1658 & 22.4 & 21.6 & $20.2-23.0$ \\
\hline Wentworth & 565 & 22.8 & 22.3 & 135 & 27.5 & 27.2 & 79 & 15.8 & 15.9 & 779 & 22.5 & 22.0 & $20.0-24.2$ \\
\hline \multicolumn{14}{|l|}{ South Western } \\
\hline Sydney & 1420 & 23.1 & 22.0 & 274 & 22.1 & 21.8 & 152 & 11.9 & 11.9 & 1846 & 21.3 & 20.5 & $19.3-21.8$ \\
\hline Central Coast & 457 & 24.0 & 24.0 & 81 & 21.7 & 21.2 & 50 & 13.0 & 12.8 & 588 & 22.1 & 22.0 & $19.7-24.5$ \\
\hline Hunter & 948 & 26.5 & 25.9 & 234 & 33.0 & 32.3 & 104 & 14.7 & 14.9 & 1286 & 25.8 & 25.3 & $23.5-27.2$ \\
\hline Illawarra & 497 & 22.0 & 21.2 & 85 & 19.0 & 18.6 & 68 & 15.1 & 15.5 & 650 & 20.6 & 19.9 & $17.9-22.1$ \\
\hline \multicolumn{14}{|l|}{ South Eastern } \\
\hline Sydney & 1167 & 25.8 & 23.7 & 243 & 25.4 & 24.0 & 115 & 11.6 & 11.5 & 1525 & 23.6 & 22.0 & $20.3-23.7$ \\
\hline Northern Rivers & 282 & 18.9 & 19.3 & 53 & 18.0 & 17.6 & 43 & 15.4 & 16.0 & 378 & 18.3 & 18.6 & $16.2-21.3$ \\
\hline Mid North Coast & 345 & 22.4 & 22.2 & 69 & 23.4 & 22.2 & 47 & 16.5 & 16.2 & 461 & 21.8 & 21.5 & $18.9-24.3$ \\
\hline New England & 292 & 23.3 & 23.6 & 52 & 21.9 & 22.4 & 33 & 14.4 & 14.5 & 377 & 21.9 & 22.2 & $19.3-25.3$ \\
\hline Macquarie & 213 & 25.5 & 25.1 & 29 & 18.0 & 18.2 & 20 & 12.3 & 11.5 & 262 & 22.6 & 22.2 & $18.8-26.2$ \\
\hline Mid Western & 251 & 20.6 & 20.1 & 59 & 25.3 & 25.5 & 35 & 15.1 & 15.5 & 345 & 20.5 & 20.2 & $17.4-23.2$ \\
\hline Far West & 63 & 21.3 & 21.1 & 10 & 18.6 & 18.7 & 3 & 5.6 & 5.5 & 76 & 18.9 & 18.9 & $13.3-25.9$ \\
\hline Greater Murray & 353 & 20.5 & 20.3 & 52 & 19.6 & 19.7 & 28 & 11.0 & 10.3 & 433 & 19.3 & 19.1 & $16.8-21.7$ \\
\hline Southern & 222 & 23.1 & 21.1 & 42 & 22.5 & 20.3 & 20 & 11.2 & 10.9 & 284 & 21.4 & 19.6 & $16.6-23.0$ \\
\hline TOTAL NSW & 10168 & 23.5 & 22.5 & 2044 & 23.6 & 22.7 & 1170 & 13.4 & 13.3 & 13382 & 22.1 & 21.2 & 20.7-21.7 \\
\hline
\end{tabular}

Source: NSW Birth Defects Register. Epidemiology and Surveillance Branch, NSW Department of Health.

\# Cases exclude terminations of pregnancy, stillbirths and livebirths where the place of residence is unknown. For 1994-1999, cases reported during pregnancy and up to one year of age are included. For 2000, cases reported during pregnancy or at birth are reported.

\section{Reference}

1. British Paediatric Association. British Paediatric Association Classification of Diseases. London: British Paediatric Association, 1979. 


\subsection{ONSET AND AUGMENTATION OF LABOUR IN SELECTED HOSPITALS}

Table 105 gives onset or augmentation of labour for individual hospitals where the number of reported deliveries exceeded 200 in 2000, totals for all hospitals within each health area and the NSW total.

\section{TABLE 105}

CONFINEMENTS BY ONSET AND AUGMENTATION OF LABOUR AND HOSPITAL, NSW 2000\#

\begin{tabular}{|c|c|c|c|c|c|c|c|c|c|c|c|c|c|c|c|c|c|c|c|c|}
\hline \multirow[t]{2}{*}{$\begin{array}{l}\text { Health Area and } \\
\text { Hospital }\end{array}$} & \multicolumn{4}{|c|}{$\begin{array}{l}\text { Spontaneous Spontaneous } \\
\text { augmented } \\
\text { with ARM }\end{array}$} & \multicolumn{2}{|c|}{$\begin{array}{c}\text { Spontaneous } \\
\text { augmented } \\
\text { oxytocics- } \\
\text { prostagl. }\end{array}$} & \multicolumn{6}{|c|}{$\begin{array}{l}\text { Onset and augmentation of } \\
\text { o labour Induced- Induced- } \\
\text { oxytocics- ARM } \\
\text { prostagl. only }\end{array}$} & \multicolumn{2}{|c|}{$\begin{array}{l}\text { labour } \\
\text { Induced- } \\
\text { ARM+ } \\
\text { oxytocics- } \\
\text { prostagl. }\end{array}$} & \multicolumn{2}{|c|}{$\begin{array}{l}\text { Induced- } \\
\text { other }\end{array}$} & \multicolumn{2}{|c|}{$\begin{array}{c}\text { Not } \\
\text { stated }\end{array}$} & \multicolumn{2}{|c|}{ TOTAL } \\
\hline & No. & $\%$ & No. & $\%$ & No. & $\%$ & No. & $\%$ & No. & $\%$ & No. & $\%$ & & & No. & $\%$ & No. & $\%$ & No. & $\%$ \\
\hline \multicolumn{21}{|l|}{ Central Sydney } \\
\hline Canterbury* & 953 & 62.2 & 0 & 0.0 & 187 & 12.2 & 121 & 7.9 & 256 & 16.7 & 14 & 40.9 & 0 & 0.0 & 2 & 0.1 & 0 & 0.0 & 1533 & 100.0 \\
\hline King George V & 2115 & 54.7 & 0 & 0.0 & 504 & 13.0 & 475 & 12.3 & 741 & 19.2 & 29 & 90.7 & 0 & 0.0 & 4 & 0.1 & 0 & 0.0 & 3868 & 100.0 \\
\hline NSW Private & 94 & 36.3 & 18 & 6.9 & 27 & 10.4 & 39 & 15.1 & 39 & 15.1 & & 62.3 & 36 & 13.9 & 0 & 0.0 & 0 & 0.0 & 259 & 100.0 \\
\hline ALL HOSPITALS & 3162 & 55.9 & 18 & 0.3 & 718 & 12.7 & 635 & 11.2 & 1036 & 18.3 & 49 & 90.9 & 36 & 0.6 & 6 & 0.1 & 0 & 0.0 & 5660 & 100.0 \\
\hline Northern Sydney & & & & & & & & & & & & & & & & & & & & \\
\hline Hornsby & 584 & 49.3 & 28 & 2.4 & 126 & 10.6 & 137 & 11.6 & 84 & 7.1 & & 50.4 & 220 & 18.6 & 0 & 0.0 & 0 & 0.0 & 1184 & 100.0 \\
\hline Manly & 461 & 50.6 & 20 & 2.2 & 85 & 9.3 & 104 & 11.4 & 74 & 8.1 & 26 & 62.9 & 141 & 15.5 & 0 & 0 & 0 & 0.0 & 911 & 100.0 \\
\hline Mona Vale & 359 & 54.1 & 6 & 0.9 & 79 & 11.9 & 70 & 10.5 & 37 & 5.6 & & 0.0 & 113 & 17.0 & 0 & 0.0 & 0 & 0.0 & 664 & 100.0 \\
\hline Royal & 837 & 48.9 & 53 & 3.1 & 205 & 12.0 & 264 & 15.4 & 117 & 6.8 & & 50.3 & 228 & 13.3 & 1 & 1 & 0 & 0 & 1710 & 100.0 \\
\hline Ryde & 413 & 56.9 & 17 & 2.3 & 75 & 10.3 & 69 & 9.5 & 57 & 7.9 & 3 & 30.4 & 92 & 12.7 & 0 & 0 & 0 & .0 & 726 & 100.0 \\
\hline Mate & 609 & 33.1 & 140 & 7.6 & 268 & 14.5 & 346 & 18.8 & 165 & 9.0 & 59 & 93.2 & 253 & 13.7 & 0 & 0 & 2 & 1 & 1842 & 100.0 \\
\hline North & 639 & 34.7 & 78 & 4.2 & 184 & 10.0 & 399 & 21.6 & 111 & 6.0 & 35 & 51.9 & 385 & 20.9 & 12 & 0.7 & 0 & .0 & 1843 & 100.0 \\
\hline Sydr & 504 & 23.2 & 289 & 13.3 & 331 & 15.2 & 365 & 16.8 & 116 & 5.3 & 34 & 41.6 & 537 & 24.7 & 1 & 0 & 0 & 0 & 2177 & 100.0 \\
\hline ALL HOSPITALS & 4406 & 39.8 & 631 & 5.7 & 1353 & 12.2 & 1754 & 15.9 & 761 & 6.9 & 167 & 71.5 & 1969 & 17.8 & 14 & 1 & 2 & .0 & 11057 & 100.0 \\
\hline West & & & & & & & & & & & & & & & & & & & & \\
\hline Aubu & 813 & 56.9 & 114 & 8.0 & 160 & 11.2 & 94 & 6.6 & 97 & 6.8 & & 70.5 & 145 & 10.1 & 0 & 0.0 & 0 & u & 1430 & 100.0 \\
\hline Blac & 1302 & 47.4 & 365 & 13.3 & 240 & 8.7 & 217 & 7.9 & 191 & 7.0 & & 50.2 & 405 & 14.8 & 16 & 0.6 & 4 & 1 & 2745 & 100.0 \\
\hline Wes & 2030 & 52.5 & 179 & 4.6 & 537 & 13.9 & 376 & 9.7 & 158 & 4.1 & 25 & 50.6 & 549 & 14.2 & 13 & 0.3 & 0 & .0 & 3867 & 100.0 \\
\hline The & 381 & 27.5 & 174 & 12.6 & 196 & 14.2 & 190 & 13.7 & 121 & 8.7 & 11 & 10.8 & 312 & 22.5 & 0 & 0 & 0 & .0 & 1385 & 100.0 \\
\hline Other & 46 & 37.1 & 14 & 11.3 & 7 & 5.6 & 18 & 14.5 & 15 & 12.1 & 1 & $\begin{array}{ll}1 & 0.8\end{array}$ & 23 & 18.5 & 0 & 0 & 0 & .0 & 124 & 100.0 \\
\hline ALL 1 & 4572 & 47.9 & 846 & 8.9 & 40 & 11.9 & 895 & 9.4 & 582 & 6.1 & 49 & 90.5 & 434 & 15.0 & 29 & 3 & 4 & .0 & 9551 & 100.0 \\
\hline Wentwc & & & & & & & & & & & & & & & & & & & & \\
\hline Blc & 5 & 59.4 & 37 & 8.3 & 38 & 8.5 & 24 & 4 & 14 & 9 & 17 & $\begin{array}{ll}7 & 3.8\end{array}$ & 18 & 4.0 & 3 & 0 & 0 & J & 446 & 100.0 \\
\hline & 1829 & 52.9 & 102 & 3.0 & 262 & 7.6 & 433 & 12.5 & 205 & 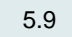 & 27 & $70 . \varepsilon$ & 589 & 17.0 & 9 & 3 & 0 & 0 & 3456 & 100.0 \\
\hline & & 35 & 74 & 17.7 & 34 & 8.1 & 48 & 11.5 & 40 & & 12 & 22 & 61 & 14.6 & 0 & & 0 & & 418 & \\
\hline $\mathrm{Ha}$ & 493 & 46.7 & 160 & 15.2 & 79 & 7.5 & 83 & 7.9 & 93 & 8. & 21 & 12.0 & 127 & 12.0 & 0 & 0.0 & 0 & .0 & 1056 & 100.0 \\
\hline & 79 & 34.6 & 35 & 15.4 & 21 & 92 & 28 & 12.3 & 20 & 8 & & 62 & 38 & 16.7 & 1 & & 0 & & 228 & 100.0 \\
\hline ALL & 2815 & 50.2 & 408 & 7.3 & 434 & 7.7 & 616 & 11.0 & 402 & 7. & 83 & 31 & 833 & 14.9 & 13 & 0.2 & 0 & 0.0 & 5604 & 100.0 \\
\hline Sout & & & & & & & & & & & & & & & & & & & & \\
\hline & 13 & 62 & 63 & 2.9 & 163 & 7.6 & 143 & 6.6 & 111 & 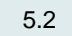 & & $\begin{array}{ll}50.2 \\
\end{array}$ & 306 & 14.2 & 11 & 0.5 & 0 & 0.0 & 55 & 100.0 \\
\hline & & 5 & 231 & 7. & 322 & 10.2 & 256 & & 208 & & 34 & & 393 & 12.4 & 30 & & 0 & & 59 & \\
\hline Cam & 1470 & 52. & 172 & 6.1 & 216 & 7.7 & 223 & 7.9 & 134 & 4. & 54 & 41. & 525 & 18.7 & 12 & 0 & 0 & 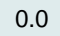 & 806 & 100.0 \\
\hline & & 52 & 172 & 9.1 & 189 & 10.0 & 150 & & 141 & & & 60. & 225 & 12.0 & 5 & & 0 & & 1882 & \\
\hline Ban & 100 & 24.9 & 33 & 8.2 & 60 & 14.9 & 64 & 15.9 & 49 & 12.2 & & 61. & 90 & 22.4 & 0 & 0 & 0 & 0.0 & 402 & 100.0 \\
\hline Syd & te 131 & 28.2 & 37 & 80 & 60 & 12.9 & 53 & 11.4 & 56 & 12.0 & 11 & 12. & 116 & & 1 & & 0 & & 65 & 100.0 \\
\hline Bowra & 378 & 57.9 & 14 & 2.1 & 32 & 4.9 & 48 & 7.4 & 48 & 7.4 & 16 & 62.5 & 108 & 16.5 & 9 & 4 & 0 & 0 & 653 & 100.0 \\
\hline ALL & & 53.0 & 722 & 6.3 & 1042 & 90 & 937 & 81 & 747 & 6 & 132 & 21.1 & 1763 & 15.3 & 68 & & 0 & 0 & 11522 & 100.0 \\
\hline Cent & & & & & & & & & & & & & & & & & & & & \\
\hline & & & 3 & 1 & 3 & 16 & 237 & 9.9 & 202 & & 20 & 0 & 363 & 15 & 0 & & 0 & & 2391 & 1 \\
\hline & & 62 & 144 & 32.4 & 2 & 4. & 3 & 7 & 0 & & & 00 & 0 & & 0 & & 0 & & 445 & \\
\hline & 196 & 27 & 60 & 8. & 103 & 14.5 & 112 & 15.8 & 73 & 10. & 25 & 53 & 139 & 19.6 & 2 & & 0 & & 710 & \\
\hline ALL & 1327 & 37.4 & 525 & 14.8 & 518 & 14.6 & 352 & 9.9 & 275 & 7.8 & 45 & $\begin{array}{lll}5 & 1.3\end{array}$ & 502 & 14.2 & 2 & & 0 & 0.0 & 3546 & 100.0 \\
\hline & & & & & & & & & & & & & & & & & & & & \\
\hline & & 57 & 3 & & 6 & 4.7 & 160 & 12.5 & 108 & & 32 & 22 & 140 & 10 & 8 & & 0 & & 1282 & \\
\hline $\mathrm{N}$ & 1 & 52 & 3 & 13 & 9 & 4. & 9 & 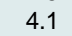 & 33 & 15 & & 41 & 18 & & 0 & & 0 & & 217 & 10 \\
\hline & & 44 & 76 & 11.5 & 52 & 7. & 68 & 10.3 & 41 & 6 & 17 & 72 & 116 & 17.5 & 1 & & 0 & 0 & 63 & \\
\hline & 2177 & 61 & 9 & 2.8 & 144 & 4. & 346 & 98 & 241 & 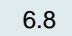 & 87 & 72 & 426 & 12.0 & 22 & & 0 & & 3541 & 10 \\
\hline & & 48 & 33 & 3. & ? & 4 & 155 & 16.1 & 86 & & 22 & 22 & 159 & 16.6 & 3 & & 0 & & 60 & 100.0 \\
\hline Oth & 235 & 52 & 40 & 9. & 19 & 4. & 78 & 17.6 & 50 & 11.3 & & 51. & 17 & 3.8 & 0 & & 0 & & 444 & 100.0 \\
\hline ALL & 4021 & 56.6 & 311 & 4.4 & 323 & 4.5 & 816 & 11.5 & 559 & 7.9 & 167 & 72.3 & 876 & 12.3 & 34 & .5 & 0 & 0.0 & 7107 & 100.0 \\
\hline Illaw & & & & & & & & & & & & & & & & & & & & \\
\hline & & & & & & & & & & & & & 10 & & 6 & & 0 & & 838 & \\
\hline & 990 & 39 & 358 & 14.4 & 285 & 11.5 & 196 & 9 & 193 & 7.8 & 35 & 51. & 427 & 17.2 & 1 & & 0 & & 2485 & 100.0 \\
\hline & 198 & 26 & 115 & 15.5 & 65 & 8.7 & 115 & 15.5 & 46 & 6 & 12 & 21. & 191 & 25.7 & 2 & & 0 & & 744 & 100.0 \\
\hline Othe & 45 & 36. & 18 & 14.8 & 9 & 7.4 & 23 & 18.9 & 14 & 11.5 & 1 & 10.8 & 12 & 9.8 & 0 & & 0 & 0.0 & 122 & 100.0 \\
\hline ALL HOSPITALS & 1762 & 42.1 & 516 & 12.3 & 379 & 9.0 & 426 & 10.2 & 344 & 8.2 & 53 & $\begin{array}{ll}3 & 1.3\end{array}$ & 700 & 16.7 & 9 & 0.2 & 0 & 0.0 & 4189 & 100.0 \\
\hline
\end{tabular}


TABLE 105 (continued)

CONFINEMENTS BY ONSET AND AUGMENTATION OF LABOUR AND HOSPITAL, NSW 2000"

\begin{tabular}{|c|c|c|c|c|c|c|c|c|c|c|c|c|c|c|c|c|c|c|c|c|c|}
\hline \multirow[t]{2}{*}{$\begin{array}{l}\text { Health Area and } \\
\text { Hospital }\end{array}$} & \multicolumn{4}{|c|}{$\begin{array}{r}\text { Spontaneous Spontaneous } \\
\text { augmented } \\
\text { with ARM }\end{array}$} & \multicolumn{9}{|c|}{$\begin{array}{l}\text { Onset and augmentation of labour } \\
\text { Spontaneous No labour Induced- Induced- } \\
\begin{array}{lll}\text { augmented } & \text { oxytocics- ARM } \\
\text { oxytocics- } & \text { prostagl. } & \text { only }\end{array}\end{array}$} & \multicolumn{2}{|c|}{$\begin{array}{l}\text { Induced- } \\
\text { ARM+ } \\
\text { oxytocics- } \\
\text { prostagl. }\end{array}$} & \multicolumn{2}{|c|}{$\begin{array}{l}\text { Induced- } \\
\text { other"\# }\end{array}$} & \multicolumn{2}{|c|}{$\begin{array}{c}\text { Not } \\
\text { stated }\end{array}$} & \multicolumn{2}{|c|}{ TOTAL } \\
\hline & No. & $\%$ & No. & $\%$ & No. & $\pi$ & No & $\%$ & No. & $\%$ & No & & $\%$ & No. & $\%$ & No. & $\%$ & No. & $\%$ & No. & $\%$ \\
\hline \multicolumn{22}{|l|}{ South Eastern Sydney } \\
\hline $\begin{array}{l}\text { Royal Hospital } \\
\text { for Women }\end{array}$ & 1678 & 43.4 & 240 & 6.2 & 687 & 17.8 & 443 & 11.5 & 261 & 6.8 & 19 & 90.5 & 5 & 524 & 13.6 & 10 & 0.3 & 0 & 0.0 & 3862 & 100.0 \\
\hline St. George & 1127 & 47.0 & 257 & 10.7 & 327 & 13.6 & 197 & 8.2 & 265 & 11.1 & 29 & 91.2 & 2 & 195 & 8.1 & 1 & 0.0 & 0 & 0.0 & 2398 & 100.0 \\
\hline Sutherland & 401 & 42.1 & 71 & 7.5 & 132 & 13.9 & 85 & 8.9 & 114 & 12.0 & 9 & 90.9 & 9 & 139 & 14.6 & 2 & 0.2 & 0 & 0.0 & 953 & 100.0 \\
\hline Hurstville Community & 228 & 27.2 & 70 & 8.3 & 160 & 19.1 & 143 & 17.0 & 49 & 5.8 & 7 & 70.8 & 8 & 182 & 21.7 & 0 & 0.0 & 0 & 0.0 & 839 & 100.0 \\
\hline Kareena Private & 127 & 20.6 & 48 & 7.8 & 82 & 13.3 & 121 & 19.6 & 61 & 9.9 & 8 & 31.3 & 3 & 169 & 27.4 & 1 & 0.2 & 0 & 0.0 & 617 & 100.0 \\
\hline St. George Private & 425 & 33.3 & 121 & 9.5 & 180 & 14.1 & 245 & 19.2 & 152 & 11.9 & 19 & 91.5 & 5 & 133 & 10.4 & 1 & 0.1 & 0 & 0.0 & 1276 & 100.0 \\
\hline Prince of Wales Private & 473 & 28.9 & 189 & 11.5 & 233 & 14.2 & 354 & 21.6 & 166 & 10.1 & 35 & 52.1 & 1 & 186 & 11.4 & 2 & 0.1 & 0 & 0.0 & 1638 & 100.0 \\
\hline ALL HOSPITALS & 4459 & 38.5 & 996 & 8.6 & 1801 & 15.5 & 1588 & 13.7 & 1068 & 9.2 & 126 & 1.1 & .1 & 1528 & 13.2 & 17 & 0.1 & 0 & 0.0 & 11583 & 100.0 \\
\hline Northern Rivers & & & & & & & & & & & & & & & & & & & & & \\
\hline Grafton Base & 163 & 34.6 & 59 & 12.5 & 52 & 11.0 & 70 & 14.9 & 51 & 10.8 & 8 & 31.7 & 7 & 68 & 14.4 & 0 & 0.0 & 0 & 0.0 & 471 & 100.0 \\
\hline Lismore Base & 610 & 50.3 & 106 & 8.7 & 96 & 7.9 & 123 & 10.1 & 118 & 9.7 & 31 & 2.6 & 6 & 125 & 10.3 & 3 & 0.2 & 0 & 0.0 & 1212 & 100.0 \\
\hline Murwillumbah & 162 & 35.2 & 71 & 15.4 & 69 & 15.0 & 39 & 8.5 & 67 & 14.6 & 1 & 10.2 & .2 & 50 & 10.9 & 1 & 0.2 & 0 & 0.0 & 460 & 100.0 \\
\hline Tweed Heads & 338 & 46.5 & 95 & 13.1 & 82 & 11.3 & 74 & 10.2 & 39 & 5.4 & 4 & 40.6 & 6 & 95 & 13.1 & 0 & 0.0 & 0 & 0.0 & 727 & 100.0 \\
\hline Other Area hospitals & 221 & 66.4 & 44 & 13.2 & 15 & 4.5 & 28 & 8.4 & 7 & 2.1 & 8 & 32.4 & 4 & 10 & 3.0 & 0 & 0.0 & 0 & 0.0 & 333 & 100.0 \\
\hline ALL HOSPITALS & 1494 & 46.6 & 375 & 11.7 & 314 & 9.8 & 334 & 10.4 & 282 & 8.8 & 52 & 21.6 & 6 & 348 & 10.9 & 4 & 0.1 & 0 & 0.0 & 3203 & 100.0 \\
\hline Mid North Coast & & & & & & & & & & & & & & & & & & & & & \\
\hline Coffs Harbour & 282 & 40.5 & 38 & 5.5 & 32 & 4.6 & 142 & 20.4 & 68 & 9.8 & 6 & 0.9 & 9 & 128 & 18.4 & 0 & 0.0 & 0 & 0.0 & 696 & 100.0 \\
\hline Kempsey & 112 & 38.2 & 72 & 24.6 & 19 & 6.5 & 30 & 10.2 & 21 & 7.2 & 2 & 20.7 & .7 & 37 & 12.6 & 0 & 0.0 & 0 & 0.0 & 293 & 100.0 \\
\hline Port Macquarie Base & 240 & 34.0 & 123 & 17.4 & 62 & 8.8 & 91 & 12.9 & 70 & 9.9 & 5 & 50.7 & .7 & 112 & 15.9 & 2 & 0.3 & 0 & 0.0 & 705 & 100.0 \\
\hline Manning Base & 322 & 45.4 & 89 & 12.6 & 54 & 7.6 & 67 & 9.4 & 57 & 8.0 & 16 & 2.3 & 3 & 104 & 14.7 & 0 & 0.0 & 0 & 0.0 & 709 & 100.0 \\
\hline Other Area hospitals & 140 & 50.5 & 29 & 10.5 & 26 & 9.4 & 36 & 13.0 & 19 & 6.9 & 2 & 20.7 & .7 & 25 & 9.0 & 0 & 0.0 & 0 & 0.0 & 277 & 100.0 \\
\hline ALL HOSPITALS & 1096 & 40.9 & 351 & 13.1 & 193 & 7.2 & 366 & 13.7 & 235 & 8.8 & 31 & 1.2 & 2 & 406 & 15.1 & 2 & 0.1 & 0 & 0.0 & 2680 & 100.0 \\
\hline New England & & & & & & & & & & & & & & & & & & & & & \\
\hline Armidale & 148 & 32.8 & 74 & 16.4 & 75 & 16.6 & 25 & 5.5 & 63 & 14.0 & 9 & 92.0 & 0 & 57 & 12.6 & 0 & 0.0 & 0 & 0.0 & 451 & 100.0 \\
\hline Inverell & 75 & 32.6 & 35 & 15.2 & 23 & 10.0 & 35 & 15.2 & 26 & 11.3 & 5 & 2.2 & 2 & 31 & 13.5 & 0 & 0.0 & 0 & 0.0 & 230 & 100.0 \\
\hline Moree & 118 & 48.6 & 23 & 9.5 & 17 & 7.0 & 21 & 8.6 & 18 & 7.4 & 1 & 10.4 & 4 & 45 & 18.5 & 0 & 0.0 & 0 & 0.0 & 243 & 100.0 \\
\hline Tamworth Base & 173 & 27.2 & 130 & 20.5 & 64 & 10.1 & 93 & 14.6 & 38 & 6.0 & 11 & 1.7 & .7 & 125 & 19.7 & 1 & 0.2 & 0 & 0.0 & 635 & 100.0 \\
\hline Other Area hospitals & 267 & 36.5 & 104 & 14.2 & 54 & 7.4 & 82 & 11.2 & 108 & 14.8 & 13 & 31.8 & 8 & 100 & 13.7 & 3 & 0.4 & 0 & 0.0 & 731 & 100.0 \\
\hline ALL HOSPITALS & 781 & 34.1 & 366 & 16.0 & 233 & 10.2 & 256 & 11.2 & 253 & 11.0 & 39 & 91.7 & .7 & 358 & 15.6 & 4 & 0.2 & 0 & 0.0 & 2290 & 100.0 \\
\hline Macquarie & & & & & & & & & & & & & & & & & & & & & \\
\hline Dubbo Base & 542 & 43.9 & 144 & 11.7 & 125 & 10.1 & 114 & 9.2 & 112 & 9.1 & 36 & 2.9 & 9 & 160 & 13.0 & 2 & 0.2 & 0 & 0.0 & 1235 & 100.0 \\
\hline Mudgee & 127 & 52.5 & 21 & 8.7 & 23 & 9.5 & 32 & 13.2 & 20 & 8.3 & 4 & 41.7 & .7 & 15 & 6.2 & 0 & 0.0 & 0 & 0.0 & 242 & 100.0 \\
\hline Other Area hospitals & 100 & 64.5 & 29 & 18.7 & 2 & 1.3 & 20 & 12.9 & 0 & 0.0 & 1 & 10.6 & 6 & 3 & 1.9 & 0 & 0.0 & 0 & 0.0 & 155 & 100.0 \\
\hline ALL HOSPITALS & 769 & 47.1 & 194 & 11.9 & 150 & 9.2 & 166 & 10.2 & 132 & 8.1 & 41 & 2.5 & 5 & 178 & 10.9 & 2 & 0.1 & 0 & 0.0 & 1632 & 100.0 \\
\hline Mid Western & & & & & & & & & & & & & & & & & & & & & \\
\hline st Base & 258 & 49.0 & 58 & 11.0 & 37 & 7.0 & 73 & 13.9 & 58 & 11.0 & 8 & 31.5 & 5 & 35 & 6.6 & 0 & 0.0 & 0 & 0.0 & 527 & 100.0 \\
\hline Orang & 270 & 35.4 & 102 & 13.4 & 72 & 9. & 111 & 14.5 & 44 & 5 & 25 & 53.3 & 3 & 138 & 18.1 & 0 & 0.0 & 1 & 1 & 763 & 100.0 \\
\hline Parkes & 126 & 61.2 & 8 & 3.9 & 6 & 2. & 30 & 14.6 & 20 & 9 & 3 & 31.5 & 5 & 13 & 6. & 0 & 0. & 0 & 0.0 & 206 & 100.0 \\
\hline Other Area hos & 238 & 39.0 & 103 & 16.9 & 42 & 6. & 97 & 15.9 & 72 & 11. & 23 & 3.8 & 8 & 36 & 5.9 & 0 & 0. & 0 & 0.0 & 611 & 100.0 \\
\hline ALL HOSPITALS & 892 & 42.3 & 271 & 12.9 & 157 & 7.5 & 311 & 14.8 & 194 & 9.2 & 59 & 92.8 & 8 & 222 & 10.5 & 0 & 0.0 & 1 & 0.0 & 2107 & 100.0 \\
\hline Far West & & & & & & & & & & & & & & & & & & & & & \\
\hline Broken Hill Base & 183 & 66.1 & 21 & 7.6 & 10 & 3.6 & 17 & 6.1 & 13 & 4.7 & 14 & 45.1 & 1 & 19 & 6.9 & 0 & 0.0 & 0 & 0.0 & 277 & 100.0 \\
\hline Other Area hospitals & 59 & 71.1 & 5 & 6.0 & 7 & 8.4 & 3 & 3.6 & 8 & 9.6 & 0 & 0.0 & 0 & 1 & 1.2 & 0 & 0.0 & 0 & 0.0 & 83 & 100.0 \\
\hline ALL HOSPITALS & 242 & 67.2 & 26 & 7.2 & 17 & 4.7 & 20 & 5.6 & 21 & 5.8 & 14 & 43.9 & 9 & 20 & 5.6 & 0 & 0.0 & 0 & 0.0 & 360 & 100.0 \\
\hline Greater Murray & & & & & & & & & & & & & & & & & & & & & \\
\hline Griffith Base & 205 & 42.9 & 66 & 13.8 & 21 & 4.4 & 58 & 12.1 & 79 & 16.5 & 8 & 31.7 & .7 & 41 & 8.6 & 0 & 0.0 & 0 & 0.0 & 478 & 100.0 \\
\hline Wagga Wagga Base & 393 & 47.9 & 73 & 8.9 & 57 & 7.0 & 93 & 11.3 & 89 & 10.9 & 25 & 53.0 & 0 & 81 & 9.9 & 9 & 1.1 & 0 & 0.0 & 820 & 100.0 \\
\hline Calvary, Wagga Wagga & 137 & 31.8 & 19 & 4.4 & 28 & 6.5 & 54 & 12.5 & 123 & 28.5 & 16 & 3.7 & .7 & 53 & 12.3 & 1 & 0.2 & 0 & 0.0 & 431 & 100.0 \\
\hline Other Area hospitals & 440 & 50.6 & 103 & 11.9 & 30 & 3.5 & 85 & 9.8 & 131 & 15.1 & 19 & 2.2 & 2 & 61 & 7.0 & 0 & 0.0 & 0 & 0.0 & 869 & 100.0 \\
\hline ALL HOSPITALS & 1175 & 45.2 & 261 & 10.0 & 136 & 5.2 & 290 & 11.2 & 422 & 16.2 & 68 & 2.6 & 6 & 236 & 9.1 & 10 & 0.4 & 0 & 0.0 & 2598 & 100.0 \\
\hline Sou & & & & & & & & & & & & & & & & & & & & & \\
\hline Bega & 86 & 41.0 & 35 & 16.7 & 19 & 9. & 29 & 13.8 & 16 & 7. & 1 & 10.5 & 5 & 23 & 11.0 & 1 & 0.5 & 0 & 0.0 & 210 & 100.0 \\
\hline Goulburn Base & 165 & 52.2 & 38 & 12.0 & 38 & 12.0 & 41 & 13.0 & 26 & 8.2 & 0 & 0.0 & 0 & 7 & 2.2 & 0 & 0.0 & 1 & 0.3 & 316 & 100.0 \\
\hline Queanbeyan & 190 & 56.7 & 27 & 8.1 & 28 & 8.4 & 23 & 6.9 & 50 & 14.9 & 3 & 30.9 & 9 & 14 & 4.2 & 0 & 0.0 & 0 & 0.0 & 335 & 100.0 \\
\hline Other Area hospitals & 418 & 52.1 & 89 & 11.1 & 56 & 7.0 & 71 & 8.9 & 88 & 11.0 & 17 & 72.1 & 1 & 63 & 7.9 & 0 & 0.0 & 0 & 0.0 & 802 & 100.0 \\
\hline ALL HOSPITALS & 859 & 51.7 & 189 & 11.4 & 141 & 8.5 & 164 & 9.9 & 180 & 10.8 & 21 & 1.3 & 3 & 107 & 6.4 & 1 & 0.1 & 1 & 0.1 & 1663 & 100.0 \\
\hline TOTAL NSW & 40042 & 46.3 & 014 & 8.1 & 9050 & 10.5 & 926 & 11.5 & 7493 & 8.7 & 1196 & 61.4 & 41 & 11516 & 13.32 & 215 & 0.2 & 8 & 0.0 & 86460 & 100.0 \\
\hline
\end{tabular}

Source: NSW Midwives Data Collection (HOIST). Epidemiology and Surveillance Branch, NSW Department of Health.

\# Hospitals with more than 200 deliveries are identified individually. All hospitals include all public and private hospitals.

\#\# This category includes other forms of induction such as Foley's catheter.

King George $V$ and Canterbury Hospitals supply data electronically and reports augmentation by oxytocin-prostaglandin only. 


\subsection{TYPE OF DELIVERY IN SELECTED HOSPITALS}

Table 106 gives type of delivery for individual hospitals where the number of reported confinements exceeded 200 in 2000 , totals for all hospitals within each health area and the NSW total.

\begin{tabular}{|c|c|c|c|c|c|c|c|c|c|c|c|c|c|c|c|c|}
\hline \multirow{3}{*}{$\begin{array}{l}\text { Health Area and } \\
\text { Hospital }\end{array}$} & \multirow{2}{*}{\multicolumn{2}{|c|}{$\begin{array}{l}\text { Normal } \\
\text { vaginal }\end{array}$}} & \multirow{2}{*}{\multicolumn{2}{|c|}{ Forceps }} & \multirow{2}{*}{\multicolumn{2}{|c|}{$\begin{array}{l}\text { Vacuum } \\
\text { extraction }\end{array}$}} & \multicolumn{4}{|c|}{ Type of delivery } & \multirow{2}{*}{\multicolumn{2}{|c|}{$\begin{array}{l}\text { Emergency } \\
\text { caesarean }\end{array}$}} & \multirow{2}{*}{\multicolumn{2}{|c|}{ Not stated }} & \multirow{2}{*}{\multicolumn{2}{|c|}{ TOTAL }} \\
\hline & & & & & & & & & & & & & & & & \\
\hline & No. & $\%$ & No. & $\%$ & No. & $\%$ & No. & $\%$ & No. & $\%$ & No. & $\%$ & No. & $\%$ & No. & $\%$ \\
\hline \multicolumn{17}{|l|}{ Central Sydney } \\
\hline Canterbury & 1083 & 70.6 & 61 & 4.0 & 111 & 7.2 & 7 & 0.5 & 121 & 7.9 & 150 & 9.8 & 0 & 0.0 & 1533 & 100.0 \\
\hline King George V & 2516 & 65.0 & 123 & 3.2 & 300 & 7.8 & 50 & 1.3 & 475 & 12.3 & 404 & 10.4 & 0 & 0.0 & 3868 & 100.0 \\
\hline NSW Private & 142 & 54.8 & 18 & 6.9 & 34 & 13.1 & 0 & 0.0 & 39 & 15.1 & 26 & 10.0 & 0 & 0.0 & 259 & 100.0 \\
\hline ALL HOSPITALS & 3741 & 66.1 & 202 & 3.6 & 445 & 7.9 & 57 & 1.0 & 635 & 11.2 & 580 & 10.2 & 0 & 0.0 & 5660 & 100.0 \\
\hline \multicolumn{17}{|l|}{ Northern Sydney } \\
\hline Hornsby & 808 & 68.2 & 49 & 4.1 & 80 & 6.8 & 6 & 0.5 & 137 & 11.6 & 104 & 8.8 & 0 & 0.0 & 1184 & 100.0 \\
\hline Manly & 631 & 69.3 & 29 & 3.2 & 55 & 6.0 & 1 & 0.1 & 104 & 11.4 & 91 & 10.0 & 0 & 0.0 & 911 & 100.0 \\
\hline Mona Vale & 437 & 65.8 & 39 & 5.9 & 53 & 8.0 & 3 & 0.5 & 70 & 10.5 & 62 & 9.3 & 0 & 0.0 & 664 & 100.0 \\
\hline Royal North Shore & 970 & 56.7 & 127 & 7.4 & 101 & 5.9 & 14 & 0.8 & 264 & 15.4 & 234 & 13.7 & 0 & 0.0 & 1710 & 100.0 \\
\hline Ryde & 511 & 70.4 & 31 & 4.3 & 33 & 4.5 & 6 & 0.8 & 69 & 9.5 & 76 & 10.5 & 0 & 0.0 & 726 & 100.0 \\
\hline Mater, North Sydney & 895 & 48.6 & 102 & 5.5 & 271 & 14.7 & 2 & 0.1 & 346 & 18.8 & 224 & 12.2 & 2 & 0.1 & 1842 & 100.0 \\
\hline North Shore Private & 893 & 48.5 & 83 & 4.5 & 239 & 13.0 & 6 & 0.3 & 399 & 21.6 & 223 & 12.1 & 0 & 0.0 & 1843 & 100.0 \\
\hline Sydney Adventist & 1289 & 59.2 & 164 & 7.5 & 159 & 7.3 & 8 & 0.4 & 365 & 16.8 & 192 & 8.8 & 0 & 0.0 & 2177 & 100.0 \\
\hline ALL HOSPITALS & 6434 & 58.2 & 624 & 5.6 & 991 & 9.0 & 46 & 0.4 & 1754 & 15.9 & 1206 & 10.9 & 2 & 0.0 & 11057 & 100.0 \\
\hline Western Sydney & & & & & & & & & & & & & & & & \\
\hline Auburn & 1153 & 80.6 & 55 & 3.8 & 21 & 1.5 & 16 & 1.1 & 94 & 6.6 & 91 & 6.4 & 0 & 0.0 & 1430 & 100.0 \\
\hline Blacktown & 2024 & 73.7 & 163 & 5.9 & 83 & 3.0 & 17 & 0.6 & 217 & 7.9 & 232 & 8.5 & 9 & 0.3 & 2745 & 100.0 \\
\hline Westmead & 2605 & 67.4 & 322 & 8.3 & 103 & 2.7 & 51 & 1.3 & 376 & 9.7 & 410 & 10.6 & 0 & 0.0 & 3867 & 100.0 \\
\hline The Hills Private & 851 & 61.4 & 138 & 10.0 & 61 & 4.4 & 11 & 0.8 & 190 & 13.7 & 134 & 9.7 & 0 & 0.0 & 1385 & 100.0 \\
\hline Other Area hospitals & 76 & 61.3 & 9 & 7.3 & 11 & 8.9 & 0 & 0.0 & 18 & 14.5 & 10 & 8.1 & 0 & 0.0 & 124 & 100.0 \\
\hline ALL HOSPITALS & 6709 & 70.2 & 687 & 7.2 & 279 & 2.9 & 95 & 1.0 & 895 & 9.4 & 877 & 9.2 & 9 & 0.1 & 9551 & 100.0 \\
\hline Wentworth & & & & & & & & & & & & & & & & \\
\hline Blue Mountains & 340 & 76.2 & 4 & 0.9 & 26 & 5.8 & 6 & 1.3 & 24 & 5.4 & 44 & 9.9 & 2 & 0.4 & 446 & 100.0 \\
\hline Nepean & 2425 & 70.2 & 79 & 2.3 & 184 & 5.3 & 12 & 0.3 & 433 & 12.5 & 323 & 9.3 & 0 & 0.0 & 3456 & 100.0 \\
\hline Jamison Private & 286 & 68.4 & 26 & 6.2 & 7 & 1.7 & 1 & 0.2 & 48 & 11.5 & 50 & 12.0 & 0 & 0.0 & 418 & 100.0 \\
\hline Hawkesbury & 747 & 70.7 & 75 & 7.1 & 35 & 3.3 & 11 & 1.0 & 83 & 7.9 & 105 & 9.9 & 0 & 0.0 & 1056 & 100.0 \\
\hline Nepean Private & 160 & 70.2 & 7 & 3.1 & 4 & 1.8 & 0 & 0.0 & 28 & 12.3 & 29 & 12.7 & 0 & 0.0 & 228 & 100.0 \\
\hline ALL HOSPITALS & 3958 & 70.6 & 191 & 3.4 & 256 & 4.6 & 30 & 0.5 & 616 & 11.0 & 551 & 9.8 & 2 & 0.0 & 5604 & 100.0 \\
\hline South Western Sydney & & & & & & & & & & & & & & & & \\
\hline Fairfield & 1749 & 81.2 & 21 & 1.0 & 133 & 6.2 & 26 & 1.2 & 143 & 6.6 & 83 & 3.9 & 0 & 0.0 & 2155 & 100.0 \\
\hline Liverpool & 2359 & 74.7 & 73 & 2.3 & 186 & 5.9 & 49 & 1.6 & 256 & 8.1 & 236 & 7.5 & 0 & 0.0 & 3159 & 100.0 \\
\hline Campbelltown & 2220 & 79.1 & 35 & 1.2 & 128 & 4.6 & 16 & 0.6 & 223 & 7.9 & 184 & 6.6 & 0 & 0.0 & 2806 & 100.0 \\
\hline Bankstown-Lidcombe & 1432 & 76.1 & 27 & 1.4 & 109 & 5.8 & 13 & 0.7 & 150 & 8.0 & 151 & 8.0 & 0 & 0.0 & 1882 & 100.0 \\
\hline Bankstown Private & 220 & 54.7 & 25 & 6.2 & 48 & 11.9 & 1 & 0.2 & 64 & 15.9 & 44 & 10.9 & 0 & 0.0 & 402 & 100.0 \\
\hline Sydney Southwest & & & & & & & & & & & & & & & & \\
\hline Private & 286 & 61.5 & 20 & 4.3 & 50 & 10.8 & 6 & 1.3 & 53 & 11.4 & 50 & 10.8 & 0 & 0.0 & 465 & 100.0 \\
\hline Bowral & 423 & 64.8 & 66 & 10.1 & 73 & 11.2 & 8 & 1.2 & 48 & 7.4 & 35 & 5.4 & 0 & 0.0 & 653 & 100.0 \\
\hline ALL HOSPITALS & 8689 & 75.4 & 267 & 2.3 & 727 & 6.3 & 119 & 1.0 & 937 & 8.1 & 783 & 6.8 & 0 & 0.0 & 11522 & 100.0 \\
\hline Central Coast & & & & & & & & & & & & & & & & \\
\hline Gosford & 1567 & 65.5 & 60 & 2.5 & 239 & 10.0 & 14 & 0.6 & 237 & 9.9 & 274 & 11.5 & 0 & 0.0 & 2391 & 100.0 \\
\hline Wyong & 404 & 90.8 & 5 & 1.1 & 17 & 3.8 & 1 & 0.2 & 3 & 0.7 & 15 & 3.4 & 0 & 0.0 & 445 & 100.0 \\
\hline North Gosford Private & 383 & 53.9 & 23 & 3.2 & 88 & 12.4 & 4 & 0.6 & 112 & 15.8 & 100 & 14.1 & 0 & 0.0 & 710 & 100.0 \\
\hline ALL HOSPITALS & 2354 & 66.4 & 88 & 2.5 & 344 & 9.7 & 19 & 0.5 & 352 & 9.9 & 389 & 11.0 & 0 & 0.0 & 3546 & 100.0 \\
\hline Hunter & & & & & & & & & & & & & & & & \\
\hline Maitland & 871 & 67.9 & 31 & 2.4 & 70 & 5.5 & 11 & 0.9 & 160 & 12.5 & 139 & 10.8 & 0 & 0.0 & 1282 & 100.0 \\
\hline Muswellbrook & 183 & 84.3 & 1 & 0.5 & 4 & 1.8 & 1 & 0.5 & 9 & 4.1 & 19 & 8.8 & 0 & 0.0 & 217 & 100.0 \\
\hline Belmont & 504 & 76.0 & 8 & 1.2 & 32 & 4.8 & 2 & 0.3 & 68 & 10.3 & 49 & 7.4 & 0 & 0.0 & 663 & 100.0 \\
\hline John Hunter & 2431 & 68.7 & 100 & 2.8 & 216 & 6.1 & 59 & 1.7 & 346 & 9.8 & 389 & 11.0 & 0 & 0.0 & 3541 & 100.0 \\
\hline Christo Road Private & 554 & 57.7 & 44 & 4.6 & 93 & 9.7 & 4 & 0.4 & 155 & 16.1 & 110 & 11.5 & 0 & 0.0 & 960 & 100.0 \\
\hline Other Area hospitals & 320 & 72.1 & 6 & 1.4 & 22 & 5.0 & 2 & 0.5 & 78 & 17.6 & 16 & 3.6 & 0 & 0.0 & 444 & 100.0 \\
\hline ALL HOSPITALS & 4863 & 68.4 & 190 & 2.7 & 437 & 6.1 & 79 & 1.1 & 816 & 11.5 & 722 & 10.2 & 0 & 0.0 & 7107 & 100.0 \\
\hline Illawarra & & & & & & & & & & & & & & & & \\
\hline Shoalhaven & 613 & 73.2 & 50 & 6.0 & 0 & 0.0 & 8 & 1.0 & 92 & 11.0 & 75 & 8.9 & 0 & 0.0 & 838 & 100.0 \\
\hline Wollongong & 1824 & 73.4 & 54 & 2.2 & 173 & 7.0 & 17 & 0.7 & 196 & 7.9 & 221 & 8.9 & 0 & 0.0 & 2485 & 100.0 \\
\hline Illawarra Private & 416 & 55.9 & 16 & 2.2 & 135 & 18.1 & 2 & 0.3 & 115 & 15.5 & 60 & 8.1 & 0 & 0.0 & 744 & 100.0 \\
\hline Other Area hospitals & 85 & 69.7 & 3 & 2.5 & 3 & 2.5 & 0 & 0.0 & 23 & 18.9 & 8 & 6.6 & 0 & 0.0 & 122 & 100.0 \\
\hline ALL HOSPITALS & 2938 & 70.1 & 123 & 2.9 & 311 & 7.4 & 27 & 0.6 & 426 & 10.2 & 364 & 8.7 & 0 & 0.0 & 4189 & 100.0 \\
\hline
\end{tabular}




\section{TABLE 106 (continued)}

CONFINEMENTS BY TYPE OF DELIVERY AND HOSPITAL, NSW $2000^{\#}$

\begin{tabular}{|c|c|c|c|c|c|c|c|c|c|c|c|c|c|c|c|c|}
\hline \multirow[t]{2}{*}{$\begin{array}{l}\text { Health Area and } \\
\text { Hospital }\end{array}$} & \multicolumn{2}{|c|}{ Normal } & \multicolumn{2}{|c|}{$\begin{array}{l}\text { Forceps } \\
\text { vaginal }\end{array}$} & \multicolumn{2}{|c|}{$\begin{array}{l}\text { Vacuum } \\
\text { extraction }\end{array}$} & \multicolumn{2}{|c|}{$\begin{array}{c}\text { Type of delivery } \\
\text { Vaginal } \\
\text { breech }\end{array}$} & \multicolumn{2}{|c|}{$\begin{array}{c}\text { Elective } \\
\text { caesarean }\end{array}$} & \multicolumn{2}{|c|}{$\begin{array}{l}\text { Emergency } \\
\text { caesarean }\end{array}$} & \multicolumn{2}{|c|}{ Not stated } & \multicolumn{2}{|c|}{ TOTAL } \\
\hline & No. & $\%$ & No. & $\%$ & No. & $\%$ & No. & $\%$ & No. & $\%$ & No. & $\%$ & No. & $\%$ & No. & $\%$ \\
\hline \multicolumn{17}{|l|}{ South Eastern Sydney } \\
\hline Royal Hospital & & & & & & & & & & & & & & & & \\
\hline for Women & 2245 & 58.1 & 262 & 6.8 & 338 & 8.8 & 34 & 0.9 & 443 & 11.5 & 540 & 14.0 & 0 & 0.0 & 3862 & 100.0 \\
\hline St. George & 1582 & 66.0 & 107 & 4.5 & 180 & 7.5 & 13 & 0.5 & 197 & 8.2 & 319 & 13.3 & 0 & 0.0 & 2398 & 100.0 \\
\hline Sutherland & 669 & 70.2 & 45 & 4.7 & 48 & 5.0 & 5 & 0.5 & 85 & 8.9 & 101 & 10.6 & 0 & 0.0 & 953 & 100.0 \\
\hline Hurstville Community & 411 & 49.0 & 87 & 10.4 & 84 & 10.0 & 5 & 0.6 & 143 & 17.0 & 109 & 13.0 & 0 & 0.0 & 839 & 100.0 \\
\hline Kareena Private & 290 & 47.0 & 96 & 15.6 & 23 & 3.7 & 6 & 1.0 & 121 & 19.6 & 81 & 13.1 & 0 & 0.0 & 617 & 100.0 \\
\hline $\begin{array}{l}\text { St. George Private } \\
\text { Prince of Wales }\end{array}$ & 640 & 50.2 & 115 & 9.0 & 67 & 5.3 & 4 & 0.3 & 245 & 19.2 & 204 & 16.0 & 1 & 0.1 & 1276 & 100.0 \\
\hline Private & 827 & 50.5 & 80 & 4.9 & 191 & 11.7 & 5 & 0.3 & 354 & 21.6 & 181 & 11.1 & 0 & 0.0 & 1638 & 100.0 \\
\hline ALL HOSPITALS & 6664 & 57.5 & 792 & 6.8 & 931 & 8.0 & 72 & 0.6 & 1588 & 13.7 & 1535 & 13.3 & 1 & 0.0 & 11583 & 100.0 \\
\hline Northern Rivers & & & & & & & & & & & & & & & & \\
\hline Grafton Base & 274 & 58.2 & 40 & 8.5 & 13 & 2.8 & 3 & 0.6 & 70 & 14.9 & 71 & 15.1 & 0 & 0.0 & 471 & 100.0 \\
\hline Lismore Base & 849 & 70.0 & 41 & 3.4 & 49 & 4.0 & 10 & 0.8 & 123 & 10.1 & 140 & 11.6 & 0 & 0.0 & 1212 & 100.0 \\
\hline Murwillumbah & 332 & 72.2 & 8 & 1.7 & 14 & 3.0 & 7 & 1.5 & 39 & 8.5 & 60 & 13.0 & 0 & 0.0 & 460 & 100.0 \\
\hline Tweed Heads & 544 & 74.8 & 20 & 2.8 & 18 & 2.5 & 7 & 1.0 & 74 & 10.2 & 64 & 8.8 & 0 & 0.0 & 727 & 100.0 \\
\hline Other Area hospitals & 299 & 89.8 & 3 & 0.9 & 1 & 0.3 & 1 & 0.3 & 28 & 8.4 & 1 & 0.3 & 0 & 0.0 & 333 & 100.0 \\
\hline ALL HOSPITALS & 2298 & 71.7 & 112 & 3.5 & 95 & 3.0 & 28 & 0.9 & 334 & 10.4 & 336 & 10.5 & 0 & 0.0 & 3203 & 100.0 \\
\hline Mid North Coast & & & & & & & & & & & & & & & & \\
\hline Coffs Harbour & 418 & 60.1 & 42 & 6.0 & 14 & 2.0 & 5 & 0.7 & 142 & 20.4 & 75 & 10.8 & 0 & 0.0 & 696 & 100.0 \\
\hline Kempsey & 229 & 78.2 & 7 & 2.4 & 3 & 1.0 & 3 & 1.0 & 30 & 10.2 & 21 & 7.2 & 0 & 0.0 & 293 & 100.0 \\
\hline Port Macquarie Base & 489 & 69.4 & 27 & 3.8 & 23 & 3.3 & 3 & 0.4 & 91 & 12.9 & 72 & 10.2 & 0 & 0.0 & 705 & 100.0 \\
\hline Manning Base & 533 & 75.2 & 11 & 1.6 & 33 & 4.7 & 6 & 0.8 & 67 & 9.4 & 59 & 8.3 & 0 & 0.0 & 709 & 100.0 \\
\hline Other Area hospitals & 217 & 78.3 & 7 & 2.5 & 8 & 2.9 & 1 & 0.4 & 36 & 13.0 & 8 & 2.9 & 0 & 0.0 & 277 & 100.0 \\
\hline ALL HOSPITALS & 1886 & 70.4 & 94 & 3.5 & 81 & 3.0 & 18 & 0.7 & 366 & 13.7 & 235 & 8.8 & 0 & 0.0 & 2680 & 100.0 \\
\hline New England & & & & & & & & & & & & & & & & \\
\hline Armidale & 388 & 86.0 & 4 & 0.9 & 13 & 2.9 & 3 & 0.7 & 25 & 5.5 & 18 & 4.0 & 0 & 0.0 & 451 & 100.0 \\
\hline Inverell & 162 & 70.4 & 12 & 5.2 & 1 & 0.4 & 4 & 1.7 & 35 & 15.2 & 16 & 7.0 & 0 & 0.0 & 230 & 100.0 \\
\hline Moree & 162 & 66.7 & 17 & 7.0 & 11 & 4.5 & 0 & 0.0 & 21 & 8.6 & 32 & 13.2 & 0 & 0.0 & 243 & 100.0 \\
\hline Tamworth Base & 388 & 61.1 & 35 & 5.5 & 54 & 8.5 & 5 & 0.8 & 93 & 14.6 & 60 & 9.4 & 0 & 0.0 & 635 & 100.0 \\
\hline Other Area hospitals & 509 & 69.6 & 47 & 6.4 & 30 & 4.1 & 5 & 0.7 & 82 & 11.2 & 58 & 7.9 & 0 & 0.0 & 731 & 100.0 \\
\hline ALL HOSPITALS & 1609 & 70.3 & 115 & 5.0 & 109 & 4.8 & 17 & 0.7 & 256 & 11.2 & 184 & 8.0 & 0 & 0.0 & 2290 & 100.0 \\
\hline Macquarie & & & & & & & & & & & & & & & & \\
\hline Dubbo Base & 897 & 72.6 & 84 & 6.8 & 41 & 3.3 & 7 & 0.6 & 114 & 9.2 & 92 & 7.4 & 0 & 0.0 & 1235 & 100.0 \\
\hline Mudgee & 169 & 69.8 & 1 & 0.4 & 19 & 7.9 & 1 & 0.4 & 32 & 13.2 & 20 & 8.3 & 0 & 0.0 & 242 & 100.0 \\
\hline Other Area hospitals & 123 & 79.4 & 5 & 3.2 & 5 & 3.2 & 2 & 1.3 & 20 & 12.9 & 0 & 0.0 & 0 & 0.0 & 155 & 100.0 \\
\hline ALL HOSPITALS & 1189 & 72.9 & 90 & 5.5 & 65 & 4.0 & 10 & 0.6 & 166 & 10.2 & 112 & 6.9 & 0 & 0.0 & 1632 & 100.0 \\
\hline Mid Western & & & & & & & & & & & & & & & & \\
\hline Bathurst Base & 336 & 63.8 & 18 & 3.4 & 24 & 4.6 & 1 & 0.2 & 73 & 13.9 & 75 & 14.2 & 0 & 0.0 & 527 & 100.0 \\
\hline Orange Base & 491 & 64.4 & 44 & 5.8 & 25 & 3.3 & 5 & 0.7 & 111 & 14.5 & 87 & 11.4 & 0 & 0.0 & 763 & 100.0 \\
\hline Parkes & 137 & 66.5 & 6 & 2.9 & 5 & 2.4 & 3 & 1.5 & 30 & 14.6 & 25 & 12.1 & 0 & 0.0 & 206 & 100.0 \\
\hline Other Area hospitals & 412 & 67.4 & 10 & 1.6 & 30 & 4.9 & 4 & 0.7 & 97 & 15.9 & 58 & 9.5 & 0 & 0.0 & 611 & 100.0 \\
\hline ALL HOSPITALS & 1376 & 65.3 & 78 & 3.7 & 84 & 4.0 & 13 & 0.6 & 311 & 14.8 & 245 & 11.6 & 0 & 0.0 & 2107 & 100.0 \\
\hline Far West & & & & & & & & & & & & & & & & \\
\hline Broken Hill Base & 221 & 79.8 & 9 & 3.2 & 4 & 1.4 & 5 & 1.8 & 17 & 6.1 & 21 & 7.6 & 0 & 0.0 & 277 & 100.0 \\
\hline Other Area hospitals & 75 & 90.4 & 1 & 1.2 & 0 & 0.0 & 2 & 2.4 & 3 & 3.6 & 2 & 2.4 & 0 & 0.0 & 83 & 100.0 \\
\hline ALL HOSPITALS & 296 & 82.2 & 10 & 2.8 & 4 & 1.1 & 7 & 1.9 & 20 & 5.6 & 23 & 6.4 & 0 & 0.0 & 360 & 100.0 \\
\hline Greater Murray & & & & & & & & & & & & & & & & \\
\hline Griffith Base & 335 & 70.1 & 14 & 2.9 & 20 & 4.2 & 4 & 0.8 & 58 & 12.1 & 47 & 9.8 & 0 & 0.0 & 478 & 100.0 \\
\hline Wagga Wagga Base & 549 & 67.0 & 71 & 8.7 & 19 & 2.3 & 9 & 1.1 & 93 & 11.3 & 79 & 9.6 & 0 & 0.0 & 820 & 100.0 \\
\hline Calvary, Wagga & & & & & & & & & & & & & & & & \\
\hline Wagga & 217 & 50.3 & 52 & 12.1 & 55 & 12.8 & 9 & 2.1 & 54 & 12.5 & 44 & 10.2 & 0 & 0.0 & 431 & 100.0 \\
\hline Other Area hospitals & 646 & 74.3 & 29 & 3.3 & 33 & 3.8 & 3 & 0.3 & 85 & 9.8 & 73 & 8.4 & 0 & 0.0 & 869 & 100.0 \\
\hline ALL HOSPITALS & 1747 & 67.2 & 166 & 6.4 & 127 & 4.9 & 25 & 1.0 & 290 & 11.2 & 243 & 9.4 & 0 & 0.0 & 2598 & 100.0 \\
\hline Southern & & & & & & & & & & & & & & & & \\
\hline Bega & 126 & 60.0 & 5 & 2.4 & 22 & 10.5 & 2 & 1.0 & 29 & 13.8 & 26 & 12.4 & 0 & 0.0 & 210 & 100.0 \\
\hline Goulburn Base & 189 & 59.8 & 40 & 12.7 & 8 & 2.5 & 1 & 0.3 & 41 & 13.0 & 37 & 11.7 & 0 & 0.0 & 316 & 100.0 \\
\hline Queanbeyan & 266 & 79.4 & 7 & 2.1 & 15 & 4.5 & 1 & 0.3 & 23 & 6.9 & 23 & 6.9 & 0 & 0.0 & 335 & 100.0 \\
\hline Other Area hospitals & 609 & 75.9 & 23 & 2.9 & 36 & 4.5 & 3 & 0.4 & 71 & 8.9 & 59 & 7.4 & 1 & 0.1 & 802 & 100.0 \\
\hline ALL HOSPITALS & 1190 & 71.6 & 75 & 4.5 & 81 & 4.9 & 7 & 0.4 & 164 & 9.9 & 145 & 8.7 & 1 & 0.1 & 1663 & 100.0 \\
\hline TOTAL NSW & 58049 & 67.1 & 3904 & 4.5 & 5367 & 6.2 & 669 & 0.8 & 9926 & 11.5 & 8530 & 9.9 & 15 & 0.0 & 86460 & 100.0 \\
\hline
\end{tabular}

Source: NSW Midwives Data Collection (HOIST). Epidemiology and Surveillance Branch, NSW Department of Health.

\# Hospitals with more than 200 deliveries are identified individually. All hospitals include all public and private hospitals. 


\subsection{PAIN RELIEF IN SELECTED HOSPITALS}

Table 107 gives type of pain relief provided to women for individual hospitals where the number of reported confinements exceeded 200 in 2000, totals for all hospitals within each health area and the NSW total. In addition to the types of pain relief listed a further 21,370 (24.9 per cent) women were reported to have received local anaesthetic to the perineum, and 927 (1.1 per cent) received a pudendal block.

\section{TABLE 107}

CONFINEMENTS BY TYPE OF PAIN RELIEF AND HOSPITAL, NSW $2000^{\#}$

\begin{tabular}{|c|c|c|c|c|c|c|c|c|c|c|c|c|c|c|}
\hline \multirow{3}{*}{$\begin{array}{l}\text { Health Area and } \\
\text { Hospital }\end{array}$} & \multirow{2}{*}{\multicolumn{2}{|c|}{ Epidural }} & \multirow{2}{*}{\multicolumn{2}{|c|}{$\begin{array}{c}\text { General } \\
\text { anaesthetic }\end{array}$}} & \multicolumn{4}{|c|}{ Type of pain relief } & \multirow{2}{*}{\multicolumn{2}{|c|}{ Spinal }} & \multirow{2}{*}{\multicolumn{2}{|c|}{ Nil }} & \multirow{2}{*}{\multicolumn{2}{|c|}{ TOTAL }} \\
\hline & & & & & & otics & & & & & & & & \\
\hline & No. & $\%$ & No. & $\%$ & No. & $\%$ & No. & $\%$ & No. & $\%$ & No. & $\%$ & No. & $\%$ \\
\hline \multicolumn{15}{|l|}{ Central Sydney } \\
\hline Canterbury & 263 & 17.2 & 104 & 6.8 & 503 & 32.8 & 768 & 50.1 & 91 & 5.9 & 218 & 14.2 & 1533 & 100.0 \\
\hline King George V & 1393 & 36.0 & 248 & 6.4 & 1374 & 35.5 & 1103 & 28.5 & 130 & 3.4 & 617 & 16.0 & 3868 & 100.0 \\
\hline NSW Private & 66 & 25.5 & 22 & 8.5 & 53 & 20.5 & 147 & 56.8 & 21 & 8.1 & 19 & 7.3 & 259 & 100.0 \\
\hline ALL HOSPITALS & 1722 & 30.4 & 374 & 6.6 & 1930 & 34.1 & 2018 & 35.7 & 242 & 4.3 & 854 & 15.1 & 5660 & 100.0 \\
\hline \multicolumn{15}{|l|}{ Northern Sydney } \\
\hline Hornsby & 472 & 39.9 & 50 & 4.2 & 308 & 26.0 & 617 & 52.1 & 31 & 2.6 & 78 & 6.6 & 1184 & 100.0 \\
\hline Manly & 290 & 31.8 & 28 & 3.1 & 301 & 33.0 & 401 & 44.0 & 88 & 9.7 & 66 & 7.2 & 911 & 100.0 \\
\hline Mona Vale & 272 & 41.0 & 22 & 3.3 & 301 & 45.3 & 242 & 36.4 & 42 & 6.3 & 64 & 9.6 & 664 & 100.0 \\
\hline Royal North Shore & 675 & 39.5 & 81 & 4.7 & 509 & 29.8 & 934 & 54.6 & 297 & 17.4 & 49 & 2.9 & 1710 & 100.0 \\
\hline Ryde & 149 & 20.5 & 56 & 7.7 & 173 & 23.8 & 387 & 53.3 & 64 & 8.8 & 76 & 10.5 & 726 & 100.0 \\
\hline Mater, North Sydney & 1162 & 63.1 & 48 & 2.6 & 216 & 11.7 & 638 & 34.6 & 31 & 1.7 & 54 & 2.9 & 1842 & 100.0 \\
\hline North Shore Private & 1105 & 60.0 & 37 & 2.0 & 203 & 11.0 & 721 & 39.1 & 160 & 8.7 & 54 & 2.9 & 1843 & 100.0 \\
\hline Sydney Adventist & 1188 & 54.6 & 103 & 4.7 & 275 & 12.6 & 768 & 35.3 & 54 & 2.5 & 60 & 2.8 & 2177 & 100.0 \\
\hline ALL HOSPITALS & 5313 & 48.1 & 425 & 3.8 & 2286 & 20.7 & 4708 & 42.6 & 767 & 6.9 & 501 & 4.5 & 11057 & 100.0 \\
\hline \multicolumn{15}{|l|}{ Western Sydney } \\
\hline Auburn & 169 & 11.8 & 102 & 7.1 & 397 & 27.8 & 655 & 45.8 & 27 & 1.9 & 247 & 17.3 & 1430 & 100.0 \\
\hline Blacktown & 659 & 24.0 & 198 & 7.2 & 467 & 17.0 & 1495 & 54.5 & 66 & 2.4 & 584 & 21.3 & 2745 & 100.0 \\
\hline Westmead & 1728 & 44.7 & 249 & 6.4 & 592 & 15.3 & 1733 & 44.8 & 74 & 1.9 & 388 & 10.0 & 3867 & 100.0 \\
\hline The Hills Private & 646 & 46.6 & 90 & 6.5 & 273 & 19.7 & 537 & 38.8 & 14 & 1.0 & 81 & 5.8 & 1385 & 100.0 \\
\hline Other Area hospitals & 40 & 32.3 & 7 & 5.6 & 42 & 33.9 & 59 & 47.6 & 14 & 11.3 & 9 & 7.3 & 124 & 100.0 \\
\hline ALL HOSPITALS & 3242 & 33.9 & 646 & 6.8 & 1771 & 18.5 & 4479 & 46.9 & 195 & 2.0 & 1309 & 13.7 & 9551 & 100.0 \\
\hline \multicolumn{15}{|l|}{ Wentworth } \\
\hline Blue Mountains & 105 & 23.5 & 10 & 2.2 & 165 & 37.0 & 275 & 61.7 & 22 & 4.9 & 61 & 13.7 & 446 & 100.0 \\
\hline Nepean & 880 & 25.5 & 284 & 8.2 & 1198 & 34.7 & 1922 & 55.6 & 260 & 7.5 & 392 & 11.3 & 3456 & 100.0 \\
\hline Jamison Private & 94 & 22.5 & 18 & 4.3 & 137 & 32.8 & 255 & 61.0 & 54 & 12.9 & 42 & 10.0 & 418 & 100.0 \\
\hline Hawkesbury & 90 & 8.5 & 77 & 7.3 & 350 & 33.1 & 630 & 59.7 & 89 & 8.4 & 175 & 16.6 & 1056 & 100.0 \\
\hline Nepean Private & 50 & 21.9 & 7 & 3.1 & 70 & 30.7 & 139 & 61.0 & 27 & 11.8 & 20 & 8.8 & 228 & 100.0 \\
\hline ALL HOSPITALS & 1219 & 21.8 & 396 & 7.1 & 1920 & 34.3 & 3221 & 57.5 & 452 & 8.1 & 690 & 12.3 & 5604 & 100.0 \\
\hline \multicolumn{15}{|l|}{ South Western Sydney } \\
\hline Fairfield & 79 & 3.7 & 199 & 9.2 & 600 & 27.8 & 1189 & 55.2 & 7 & 0.3 & 386 & 17.9 & 2155 & 100.0 \\
\hline Liverpool & 580 & 18.4 & 220 & 7.0 & 1132 & 35.8 & 1749 & 55.4 & 133 & 4.2 & 305 & 9.7 & 3159 & 100.0 \\
\hline Campbelltown & 333 & 11.9 & 189 & 6.7 & 1304 & 46.5 & 1870 & 66.6 & 150 & 5.3 & 290 & 10.3 & 2806 & 100.0 \\
\hline Bankstown-Lidcombe & 236 & 12.5 & 97 & 5.2 & 582 & 30.9 & 1188 & 63.1 & 95 & 5.0 & 193 & 10.3 & 1882 & 100.0 \\
\hline Bankstown Private & 91 & 22.6 & 52 & 12.9 & 132 & 32.8 & 221 & 55.0 & 32 & 8.0 & 30 & 7.5 & 402 & 100.0 \\
\hline Sydney Southwest Private & 135 & 29.0 & 32 & 6.9 & 196 & 42.2 & 255 & 54.8 & 11 & 2.4 & 40 & 8.6 & 465 & 100.0 \\
\hline Bowral & 160 & 24.5 & 25 & 3.8 & 259 & 39.7 & 341 & 52.2 & 19 & 2.9 & 91 & 13.9 & 653 & 100.0 \\
\hline ALL HOSPITALS & 1614 & 14.0 & 814 & 7.1 & 4205 & 36.5 & 6813 & 59.1 & 447 & 3.9 & 1335 & 11.6 & 11522 & 100.0 \\
\hline Central Coast & & & & & & & & & & & & & & \\
\hline Gosford & 680 & 28.4 & 115 & 4.8 & 921 & 38.5 & 1209 & 50.6 & 288 & 12.0 & 225 & 9.4 & 2391 & 100.0 \\
\hline Wyong & 0 & 0.0 & 23 & 5.2 & 125 & 28.1 & 225 & 50.6 & 0 & 0.0 & 121 & 27.2 & 445 & 100.0 \\
\hline North Gosford Private & 374 & 52.7 & 18 & 2.5 & 138 & 19.4 & 289 & 40.7 & 41 & 5.8 & 56 & 7.9 & 710 & 100.0 \\
\hline ALL HOSPITALS & 1054 & 29.7 & 156 & 4.4 & 1184 & 33.4 & 1723 & 48.6 & 329 & 9.3 & 402 & 11.3 & 3546 & 100.0 \\
\hline Hunter & & & & & & & & & & & & & & \\
\hline Maitland & 137 & 10.7 & 103 & 8.0 & 401 & 31.3 & 729 & 56.9 & 174 & 13.6 & 169 & 13.2 & 1282 & 100.0 \\
\hline Muswellbrook & 2 & 0.9 & 5 & 2.3 & 51 & 23.5 & 125 & 57.6 & 25 & 11.5 & 60 & 27.6 & 217 & 100.0 \\
\hline Belmont & 44 & 6.6 & 36 & 5.4 & 282 & 42.5 & 432 & 65.2 & 71 & 10.7 & 77 & 11.6 & 663 & 100.0 \\
\hline John Hunter & 796 & 22.5 & 244 & 6.9 & 894 & 25.2 & 1719 & 48.5 & 307 & 8.7 & 586 & 16.5 & 3541 & 100.0 \\
\hline Christo Road Private & 314 & 32.7 & 38 & 4.0 & 198 & 20.6 & 448 & 46.7 & 128 & 13.3 & 113 & 11.8 & 960 & 100.0 \\
\hline Other Area hospitals & 57 & 12.8 & 9 & 2.0 & 75 & 16.9 & 257 & 57.9 & 38 & 8.6 & 71 & 16.0 & 444 & 100.0 \\
\hline ALL HOSPITALS & 1350 & 19.0 & 435 & 6.1 & 1901 & 26.7 & 3710 & 52.2 & 743 & 10.5 & 1076 & 15.1 & 7107 & 100.0 \\
\hline Illawarra & & & & & & & & & & & & & & \\
\hline Shoalhaven & 94 & 11.2 & 38 & 4.5 & 172 & 20.5 & 342 & 40.8 & 113 & 13.5 & 181 & 21.6 & 838 & 100.0 \\
\hline Wollongong & 460 & 18.5 & 145 & 5.8 & 622 & 25.0 & 1650 & 66.4 & 110 & 4.4 & 273 & 11.0 & 2485 & 100.0 \\
\hline Illawarra Private & 236 & 31.7 & 59 & 7.9 & 200 & 26.9 & 474 & 63.7 & 26 & 3.5 & 26 & 3.5 & 744 & 100.0 \\
\hline Other Area hospitals & 8 & 6.6 & 3 & 2.5 & 31 & 25.4 & 55 & 45.1 & 24 & 19.7 & 19 & 15.6 & 122 & 100.0 \\
\hline ALL HOSPITALS & 798 & 19.0 & 245 & 5.8 & 1025 & 24.5 & 2521 & 60.2 & 273 & 6.5 & 499 & 11.9 & 4189 & 100.0 \\
\hline
\end{tabular}




\section{TABLE 107 (continued)}

CONFINEMENTS BY TYPE OF PAIN RELIEF AND HOSPITAL, NSW 2000\#

\begin{tabular}{|c|c|c|c|c|c|c|c|c|c|c|c|c|c|c|}
\hline \multirow{3}{*}{$\begin{array}{l}\text { Health Area and } \\
\text { Hospital }\end{array}$} & \multirow{2}{*}{\multicolumn{2}{|c|}{ Epidural }} & \multirow{2}{*}{\multicolumn{2}{|c|}{$\begin{array}{c}\text { General } \\
\text { anaesthetic }\end{array}$}} & \multicolumn{4}{|c|}{ Type of pain relief } & \multirow{2}{*}{\multicolumn{2}{|c|}{ Spinal }} & \multirow{2}{*}{\multicolumn{2}{|c|}{ Nil }} & \multirow{2}{*}{\multicolumn{2}{|c|}{ TOTAL }} \\
\hline & & & & & & $\begin{array}{l}\text { M } \\
\text { otics }\end{array}$ & & & & & & & & \\
\hline & No. & $\%$ & No. & $\%$ & No. & $\%$ & No. & $\%$ & No. & $\%$ & No. & $\%$ & No. & $\%$ \\
\hline \multicolumn{15}{|l|}{ South Eastern Sydney } \\
\hline Royal Hospital for Women & 1940 & 50.2 & 79 & 2.0 & 822 & 21.3 & 1387 & 35.9 & 240 & 6.2 & 309 & 8.0 & 3862 & 100.0 \\
\hline St. George & 755 & 31.5 & 96 & 4.0 & 630 & 26.3 & 1387 & 57.8 & 138 & 5.8 & 266 & 11.1 & 2398 & 100.0 \\
\hline Sutherland & 334 & 35.0 & 27 & 2.8 & 76 & 8.0 & 520 & 54.6 & 94 & 9.9 & 138 & 14.5 & 953 & 100.0 \\
\hline Hurstville Community & 552 & 65.8 & 29 & 3.5 & 99 & 11.8 & 216 & 25.7 & 8 & 1.0 & 44 & 5.2 & 839 & 100.0 \\
\hline Kareena Private & 468 & 75.9 & 14 & 2.3 & 65 & 10.5 & 168 & 27.2 & 11 & 1.8 & 13 & 2.1 & 617 & 100.0 \\
\hline St. George Private & 763 & 59.8 & 53 & 4.2 & 278 & 21.8 & 575 & 45.1 & 31 & 2.4 & 67 & 5.3 & 1276 & 100.0 \\
\hline Prince of Wales Private & 1287 & 78.6 & 6 & 0.4 & 89 & 5.4 & 390 & 23.8 & 53 & 3.2 & 55 & 3.4 & 1638 & 100.0 \\
\hline ALL HOSPITALS & 6099 & 52.7 & 304 & 2.6 & 2059 & 17.8 & 4643 & 40.1 & 575 & 5.0 & 892 & 7.7 & 11583 & 100.0 \\
\hline \multicolumn{15}{|l|}{ Northern Rivers } \\
\hline Grafton Base & 158 & 33.5 & 46 & 9.8 & 105 & 22.3 & 205 & 43.5 & 51 & 10.8 & 73 & 15.5 & 471 & 100.0 \\
\hline Lismore Base & 391 & 32.3 & 50 & 4.1 & 335 & 27.6 & 441 & 36.4 & 94 & 7.8 & 221 & 18.2 & 1212 & 100.0 \\
\hline Murwillumbah & 59 & 12.8 & 69 & 15.0 & 154 & 33.5 & 262 & 57.0 & 5 & 1.1 & 60 & 13.0 & 460 & 100.0 \\
\hline Tweed Heads & 152 & 20.9 & 43 & 5.9 & 284 & 39.1 & 409 & 56.3 & 31 & 4.3 & 85 & 11.7 & 727 & 100.0 \\
\hline Other Area hospitals & 17 & 5.1 & 0 & 0.0 & 51 & 15.3 & 119 & 35.7 & 14 & 4.2 & 142 & 42.6 & 333 & 100.0 \\
\hline ALL HOSPITALS & 777 & 24.3 & 208 & 6.5 & 929 & 29.0 & 1436 & 44.8 & 195 & 6.1 & 581 & 18.1 & 3203 & 100.0 \\
\hline \multicolumn{15}{|l|}{ Mid North Coast } \\
\hline Coffs Harbour & 153 & 22.0 & 77 & 11.1 & 183 & 26.3 & 305 & 43.8 & 73 & 10.5 & 106 & 15.2 & 696 & 100.0 \\
\hline Kempsey & 57 & 19.5 & 12 & 4.1 & 105 & 35.8 & 165 & 56.3 & 29 & 9.9 & 60 & 20.5 & 293 & 100.0 \\
\hline Port Macquarie Base & 217 & 30.8 & 35 & 5.0 & 200 & 28.4 & 414 & 58.7 & 15 & 2.1 & 74 & 10.5 & 705 & 100.0 \\
\hline Manning Base & 94 & 13.3 & 45 & 6.3 & 326 & 46.0 & 413 & 58.3 & 67 & 9.4 & 81 & 11.4 & 709 & 100.0 \\
\hline Other Area hospitals & 18 & 6.5 & 8 & 2.9 & 59 & 21.3 & 116 & 41.9 & 25 & 9.0 & 91 & 32.9 & 277 & 100.0 \\
\hline ALL HOSPITALS & 539 & 20.1 & 177 & 6.6 & 873 & 32.6 & 1413 & 52.7 & 209 & 7.8 & 412 & 15.4 & 2680 & 100.0 \\
\hline \multicolumn{15}{|l|}{ New England } \\
\hline Armidale & 9 & 2.0 & 24 & 5.3 & 84 & 18.6 & 199 & 44.1 & 17 & 3.8 & 38 & 8.4 & 451 & 100.0 \\
\hline Inverell & 5 & 2.2 & 8 & 3.5 & 61 & 26.5 & 115 & 50.0 & 46 & 20.0 & 45 & 19.6 & 230 & 100.0 \\
\hline Moree & 32 & 13.2 & 9 & 3.7 & 43 & 17.7 & 130 & 53.5 & 39 & 16.0 & 43 & 17.7 & 243 & 100.0 \\
\hline Tamworth Base & 207 & 32.6 & 65 & 10.2 & 141 & 22.2 & 363 & 57.2 & 28 & 4.4 & 61 & 9.6 & 635 & 100.0 \\
\hline Other Area hospitals & 102 & 14.0 & 48 & 6.6 & 193 & 26.4 & 371 & 50.8 & 58 & 7.9 & 135 & 18.5 & 731 & 100.0 \\
\hline ALL HOSPITALS & 355 & 15.5 & 154 & 6.7 & 522 & 22.8 & 1178 & 51.4 & 188 & 8.2 & 322 & 14.1 & 2290 & 100.0 \\
\hline Macquarie & & & & & & & & & & & & & & \\
\hline Dubbo Base & 276 & 22.3 & 56 & 4.5 & 380 & 30.8 & 741 & 60.0 & 71 & 5.7 & 177 & 14.3 & 1235 & 100.0 \\
\hline Mudgee & 10 & 4.1 & 23 & 9.5 & 63 & 26.0 & 147 & 60.7 & 22 & 9.1 & 34 & 14.0 & 242 & 100.0 \\
\hline Other Area hospitals & 14 & 9.0 & 5 & 3.2 & 19 & 12.3 & 51 & 32.9 & 4 & 2.6 & 64 & 41.3 & 155 & 100.0 \\
\hline ALL HOSPITALS & 300 & 18.4 & 84 & 5.1 & 462 & 28.3 & 939 & 57.5 & 97 & 5.9 & 275 & 16.9 & 1632 & 100.0 \\
\hline Mid Western & & & & & & & & & & & & & & \\
\hline Bathurst Base & 138 & 26.2 & 56 & 10.6 & 77 & 14.6 & 276 & 52.4 & 7 & 1.3 & 86 & 16.3 & 527 & 100.0 \\
\hline Orange Base & 234 & 30.7 & 42 & 5.5 & 158 & 20.7 & 428 & 56.1 & 24 & 3.1 & 110 & 14.4 & 763 & 100.0 \\
\hline Parkes & 44 & 21.4 & 23 & 11.2 & 30 & 14.6 & 82 & 39.8 & 10 & 4.9 & 54 & 26.2 & 206 & 100.0 \\
\hline Other Area hospitals & 137 & 22.4 & 35 & 5.7 & 150 & 24.5 & 313 & 51.2 & 48 & 7.9 & 104 & 17.0 & 611 & 100.0 \\
\hline ALL HOSPITALS & 553 & 26.2 & 156 & 7.4 & 415 & 19.7 & 1099 & 52.2 & 89 & 4.2 & 354 & 16.8 & 2107 & 100.0 \\
\hline Far West & & & & & & & & & & & & & & \\
\hline Broken Hill Base & 27 & 9.7 & 7 & 2.5 & 65 & 23.5 & 171 & 61.7 & 20 & 7.2 & 64 & 23.1 & 277 & 100.0 \\
\hline Other Area hospitals & 0 & 0.0 & 1 & 1.2 & 14 & 16.9 & 39 & 47.0 & 5 & 6.0 & 32 & 38.6 & 83 & 100.0 \\
\hline ALL HOSPITALS & 27 & 7.5 & 8 & 2.2 & 79 & 21.9 & 210 & 58.3 & 25 & 6.9 & 96 & 26.7 & 360 & 100.0 \\
\hline Greater Murray & & & & & & & & & & & & & & \\
\hline Griffith Base & 48 & 10.0 & 17 & 3.6 & 170 & 35.6 & 259 & 54.2 & 80 & 16.7 & 85 & 17.8 & 478 & 100.0 \\
\hline Wagga Wagga Base & 208 & 25.4 & 27 & 3.3 & 221 & 27.0 & 412 & 50.2 & 82 & 10.0 & 143 & 17.4 & 820 & 100.0 \\
\hline Calvary, Wagga Wagga & 186 & 43.2 & 4 & 0.9 & 112 & 26.0 & 172 & 39.9 & 32 & 7.4 & 58 & 13.5 & 431 & 100.0 \\
\hline Other Area hospitals & 83 & 9.6 & 28 & 3.2 & 260 & 29.9 & 496 & 57.1 & 98 & 11.3 & 155 & 17.8 & 869 & 100.0 \\
\hline ALL HOSPITALS & 525 & 20.2 & 76 & 2.9 & 763 & 29.4 & 1339 & 51.5 & 292 & 11.2 & 441 & 17.0 & 2598 & 100.0 \\
\hline Southern & & & & & & & & & & & & & & \\
\hline Bega & 23 & 11.0 & 14 & 6.7 & 61 & 29.0 & 132 & 62.9 & 43 & 20.5 & 25 & 11.9 & 210 & 100.0 \\
\hline Goulburn Base & 77 & 24.4 & 32 & 10.1 & 43 & 13.6 & 160 & 50.6 & 4 & 1.3 & 47 & 14.9 & 316 & 100.0 \\
\hline Queanbeyan & 59 & 17.6 & 18 & 5.4 & 47 & 14.0 & 154 & 46.0 & 6 & 1.8 & 115 & 34.3 & 335 & 100.0 \\
\hline Other Area hospitals & 82 & 10.2 & 31 & 3.9 & 179 & 22.3 & 407 & 50.7 & 77 & 9.6 & 190 & 23.7 & 802 & 100.0 \\
\hline ALL HOSPITALS & 241 & 14.5 & 95 & 5.7 & 330 & 19.8 & 853 & 51.3 & 130 & 7.8 & 377 & 22.7 & 1663 & 100.0 \\
\hline TOTAL & 25728 & 29.8 & 4753 & 5.5 & 22654 & 26.2 & 42303 & 48.9 & 5248 & 6.1 & 10518 & 12.2 & 86460 & 100.0 \\
\hline
\end{tabular}

Source: NSW Midwives Data Collection (HOIST). Epidemiology and Surveillance Branch, NSW Department of Health.

\# Hospitals with more than 200 deliveries are identified individually. All hospitals include all public and private hospitals. 


\subsection{PERINEAL STATUS IN SELECTED HOSPITALS}

Table 108 show the perineal status in vaginal deliveries for individual hospitals where the number of reported confinements exceeded 200 in 2000, totals for all hospitals within each health area and the NSW total.

\section{TABLE 108}

CONFINEMENTS WITH VAGINAL DELIVERIES BY PERINEAL STATUS AND HOSPITAL, NSW $2000^{*}$

\begin{tabular}{|c|c|c|c|c|c|c|c|c|c|c|c|c|c|c|c|c|c|c|}
\hline \multirow{3}{*}{$\begin{array}{l}\text { Health Area and } \\
\text { Hospital }\end{array}$} & \multirow{2}{*}{\multicolumn{4}{|c|}{ Intact }} & \multirow{2}{*}{\multicolumn{2}{|c|}{$\begin{array}{l}\text { 2nd degree } \\
\text { tear }\end{array}$}} & \multicolumn{6}{|c|}{ Perineal status } & \multirow{2}{*}{\multicolumn{2}{|c|}{ Other }} & \multirow{2}{*}{\multicolumn{2}{|c|}{ Not stated }} & \multirow{2}{*}{\multicolumn{2}{|c|}{ TOTAL }} \\
\hline & & & & & & & $\begin{array}{l}\text { 3rd or } \\
\text { degree }\end{array}$ & th & Episio & omy & $\begin{array}{l}\text { Comi } \\
\text { tear } \\
\text { episio }\end{array}$ & & & & & & & \\
\hline & No. & $\%$ & No. & $\%$ & No. & $\%$ & No. & $\%$ & No. & $\%$ & No. & $\%$ & No. & $\%$ & No. & $\%$ & No. & $\%$ \\
\hline \multicolumn{19}{|l|}{ Central Sydney } \\
\hline Canterbury & 322 & 25.5 & 423 & 33.5 & 344 & 27.3 & 20 & 1.6 & 132 & 10.5 & 1 & 0.1 & 20 & 1.6 & 0 & 0.0 & 1262 & 100.0 \\
\hline King George V & 564 & 18.9 & 1127 & 37.7 & 988 & 33.1 & 58 & 1.9 & 222 & 7.4 & 4 & 0.1 & 26 & 0.9 & 0 & 0.0 & 2989 & 100.0 \\
\hline NSW Private & 41 & 21.1 & 37 & 19.1 & 42 & 21.6 & 2 & 1.0 & 65 & 33.5 & 4 & 2.1 & 3 & 1.5 & 0 & 0.0 & 194 & 100.0 \\
\hline ALL HOSPITALS & 927 & 20.9 & 1587 & 35.7 & 1374 & 30.9 & 80 & 1.8 & 419 & 9.4 & 9 & 0.2 & 49 & 1.1 & 0 & 0.0 & 4445 & 100.0 \\
\hline \multicolumn{19}{|l|}{ Northern Sydney } \\
\hline Hornsby & 166 & 17.6 & 236 & 25.0 & 310 & 32.9 & 27 & 2.9 & 101 & 10.7 & 6 & 0.6 & 97 & 10.3 & 0 & 0.0 & 943 & 100.0 \\
\hline Manly & 149 & 20.8 & 189 & 26.4 & 162 & 22.6 & 5 & 0.7 & 86 & 12.0 & 4 & 0.6 & 121 & 16.9 & 0 & 0.0 & 716 & 100.0 \\
\hline Mona Vale & 162 & 30.5 & 174 & 32.7 & 95 & 17.9 & 2 & 0.4 & 46 & 8.6 & 1 & 0.2 & 52 & 9.8 & 0 & 0.0 & 532 & 100.0 \\
\hline Royal North Shore & 158 & 13.0 & 318 & 26.2 & 387 & 31.9 & 37 & 3.1 & 195 & 16.1 & 5 & 0.4 & 112 & 9.2 & 0 & 0.0 & 1212 & 100.0 \\
\hline Ryde & 134 & 23.1 & 153 & 26.3 & 142 & 24.4 & 10 & 1.7 & 96 & 16.5 & 3 & 0.5 & 43 & 7.4 & 0 & 0.0 & 581 & 100.0 \\
\hline Mater, North Sydney & 199 & 15.7 & 277 & 21.8 & 300 & 23.6 & 6 & 0.5 & 410 & 32.3 & 49 & 3.9 & 28 & 2.2 & 1 & 0.1 & 1270 & 100.0 \\
\hline North Shore Private & 151 & 12.4 & 247 & 20.2 & 395 & 32.4 & 30 & 2.5 & 317 & 26.0 & 31 & 2.5 & 50 & 4.1 & 0 & 0.0 & 1221 & 100.0 \\
\hline Sydney Adventist & 265 & 16.4 & 369 & 22.8 & 373 & 23.0 & 9 & 0.6 & 543 & 33.5 & 34 & 2.1 & 27 & 1.7 & 0 & 0.0 & 1620 & 100.0 \\
\hline ALL HOSPITALS & 1384 & 17.1 & 1963 & 24.2 & 2164 & 26.7 & 126 & 1.6 & 1794 & 22.2 & 133 & 1.6 & 530 & 6.5 & 1 & 0.0 & 8095 & 100.0 \\
\hline \multicolumn{19}{|l|}{ Western Sydney } \\
\hline Auburn & 450 & 36.1 & 303 & 24.3 & 266 & 21.4 & 10 & 0.8 & 163 & 13.1 & 32 & 2.6 & 21 & 1.7 & 0 & 0.0 & 1245 & 100.0 \\
\hline Blacktown & 587 & 25.7 & 491 & 21.5 & 482 & 21.1 & 37 & 1.6 & 512 & 22.4 & 73 & 3.2 & 94 & 4.1 & 11 & 0.5 & 2287 & 100.0 \\
\hline Westmead & 665 & 21.6 & 920 & 29.9 & 628 & 20.4 & 42 & 1.4 & 628 & 20.4 & 16 & 0.5 & 182 & 5.9 & 0 & 0.0 & 3081 & 100.0 \\
\hline The Hills Private & 298 & 28.1 & 204 & 19.2 & 250 & 23.6 & 6 & 0.6 & 274 & 25.8 & 12 & 1.1 & 17 & 1.6 & 0 & 0.0 & 1061 & 100.0 \\
\hline Other Area hospitals & 28 & 29.2 & 20 & 20.8 & 19 & 19.8 & 1 & 1.0 & 28 & 29.2 & 0 & 0.0 & 0 & 0.0 & 0 & 0.0 & 96 & 100.0 \\
\hline ALL HOSPITALS & 2028 & 26.1 & 1938 & 24.9 & 1645 & 21.2 & 96 & 1.2 & 1605 & 20.7 & 133 & 1.7 & 314 & 4.0 & 11 & 0.1 & 7770 & 100.0 \\
\hline Wentworth & & & & & & & & & & & & & & & & & & \\
\hline Blue Mountains & 133 & 35.4 & 78 & 20.7 & 111 & 29.5 & 11 & 2.9 & 29 & 7.7 & 5 & 1.3 & 9 & 2.4 & 0 & 0.0 & 376 & 100.0 \\
\hline Nepean & 907 & 33.6 & 825 & 30.6 & 506 & 18.7 & 28 & 1.0 & 254 & 9.4 & 3 & 0.1 & 177 & 6.6 & 0 & 0.0 & 2700 & 100.0 \\
\hline Jamison Private & 65 & 20.3 & 45 & 14.1 & 92 & 28.8 & 2 & 0.6 & 100 & 31.3 & 9 & 2.8 & 7 & 2.2 & 0 & 0.0 & 320 & 100.0 \\
\hline Hawkesbury & 330 & 38.0 & 204 & 23.5 & 196 & 22.6 & 8 & 0.9 & 87 & 10.0 & 8 & 0.9 & 35 & 4.0 & 0 & 0.0 & 868 & 100.0 \\
\hline Nepean Private & 47 & 27.5 & 22 & 12.9 & 52 & 30.4 & 0 & 0.0 & 39 & 22.8 & 6 & 3.5 & 5 & 2.9 & 0 & 0.0 & 171 & 100.0 \\
\hline ALL HOSPITALS & 1482 & 33.4 & 1174 & 26.5 & 957 & 21.6 & 49 & 1.1 & 509 & 11.5 & 31 & 0.7 & 233 & 5.3 & 0 & 0.0 & 4435 & 100.0 \\
\hline South Western Sydney & & & & & & & & & & & & & & & & & & \\
\hline Fairfield & 542 & 28.1 & 465 & 24.1 & 456 & 23.6 & 23 & 1.2 & 360 & 18.7 & 4 & 0.2 & 79 & 4.1 & 0 & 0.0 & 1929 & 100.0 \\
\hline Liverpool & 769 & 28.8 & 681 & 25.5 & 543 & 20.4 & 45 & 1.7 & 472 & 17.7 & 14 & 0.5 & 143 & 5.4 & 0 & 0.0 & 2667 & 100.0 \\
\hline Campbelltown & 761 & 31.7 & 653 & 27.2 & 393 & 16.4 & 19 & 0.8 & 378 & 15.8 & 7 & 0.3 & 188 & 7.8 & 0 & 0.0 & 2399 & 100.0 \\
\hline Bankstown-Lidcombe & 365 & 23.1 & 515 & 32.6 & 342 & 21.6 & 15 & 0.9 & 281 & 17.8 & 60 & 3.8 & 3 & 0.2 & 0 & 0.0 & 1581 & 100.0 \\
\hline Bankstown Private & 49 & 16.7 & 58 & 19.7 & 59 & 20.1 & 0 & 0.0 & 123 & 41.8 & 5 & 1.7 & 0 & 0.0 & 0 & 0.0 & 294 & 100.0 \\
\hline Sydney Southwest Privat & ate 76 & 21.0 & 83 & 22.9 & 85 & 23.5 & 3 & 0.8 & 101 & 27.9 & 12 & 3.3 & 2 & 0.6 & 0 & 0.0 & 362 & 100.0 \\
\hline Bowral & 194 & 34.0 & 144 & 25.3 & 148 & 26.0 & 5 & 0.9 & 46 & 8.1 & 2 & 0.4 & 31 & 5.4 & 0 & 0.0 & 570 & 100.0 \\
\hline ALL HOSPITALS & 2756 & 28.1 & 2599 & 26.5 & 2026 & 20.7 & 110 & 1.1 & 1761 & 18.0 & $10 \overline{4}$ & 1.1 & 446 & 4.6 & 0 & 0.0 & 9802 & 100.0 \\
\hline Central Coast & & & & & & & & & & & & & & & & & & \\
\hline Gosford & 383 & 20.4 & 653 & 34.7 & 582 & 31.0 & 52 & 2.8 & 123 & 6.5 & 17 & 0.9 & 70 & 3.7 & 0 & 0.0 & 1880 & 100.0 \\
\hline Wyong & 152 & 35.6 & 147 & 34.4 & 77 & 18.0 & 5 & 1.2 & 27 & 6.3 & 2 & 0.5 & 17 & 4.0 & 0 & 0.0 & 427 & 100.0 \\
\hline North Gosford Private & 124 & 24.9 & 85 & 17.1 & 180 & 36.1 & 4 & 0.8 & 92 & 18.5 & 6 & 1.2 & 7 & 1.4 & 0 & 0.0 & 498 & 100.0 \\
\hline ALL HOSPITALS & 659 & 23.5 & 885 & 31.6 & 839 & 29.9 & 61 & 2.2 & 242 & 8.6 & 25 & 0.9 & 94 & 3.4 & 0 & 0.0 & 2805 & 100.0 \\
\hline Hunter & & & & & & & & & & & & & & & & & & \\
\hline Maitland & 344 & 35.0 & 374 & 38.0 & 147 & 15.0 & 12 & 1.2 & 63 & 6.4 & 0 & 0.0 & 43 & 4.4 & 0 & 0.0 & 983 & 100.0 \\
\hline Muswellbrook & 108 & 57.1 & 40 & 21.2 & 28 & 14.8 & 1 & 0.5 & 5 & 2.6 & 5 & 2.6 & 2 & 1.1 & 0 & 0.0 & 189 & 100.0 \\
\hline Belmont & 209 & 38.3 & 169 & 31.0 & 109 & 20.0 & 7 & 1.3 & 41 & 7.5 & 3 & 0.5 & 8 & 1.5 & 0 & 0.0 & 546 & 100.0 \\
\hline John Hunter & 858 & 30.6 & 1024 & 36.5 & 548 & 19.5 & 70 & 2.5 & 167 & 6.0 & 9 & 0.3 & 130 & 4.6 & 0 & 0.0 & 2806 & 100.0 \\
\hline Christo Road Private & 165 & 23.7 & 209 & 30.1 & 174 & 25.0 & 10 & 1.4 & 124 & 17.8 & 0 & 0.0 & 13 & 1.9 & 0 & 0.0 & 695 & 100.0 \\
\hline Other Area hospitals & 179 & 51.1 & 76 & 21.7 & 56 & 16.0 & 2 & 0.6 & 31 & 8.9 & 5 & 1.4 & 1 & 0.3 & 0 & 0.0 & 350 & 100.0 \\
\hline ALL HOSPITALS & 1863 & 33.5 & 1892 & 34.0 & 1062 & 19.1 & 102 & 1.8 & 431 & 7.7 & 22 & 0.4 & 197 & 3.5 & 0 & 0.0 & 5569 & 100.0 \\
\hline Illawarra & & & & & & & & & & & & & & & & & & \\
\hline Shoalhaven & 275 & 41.0 & 231 & 34.4 & 79 & 11.8 & 9 & 1.3 & 45 & 6.7 & 3 & 0.4 & 29 & 4.3 & 0 & 0.0 & 671 & 100.0 \\
\hline Wollongong & 831 & 40.2 & 557 & 26.9 & 438 & 21.2 & 16 & 0.8 & 172 & 8.3 & 4 & 0.2 & 50 & 2.4 & 0 & 0.0 & 2068 & 100.0 \\
\hline Illawarra Private & 125 & 22.0 & 80 & 14.1 & 158 & 27.8 & 8 & 1.4 & 178 & 31.3 & 13 & 2.3 & 7 & 1.2 & 0 & 0.0 & 569 & 100.0 \\
\hline Other Area hospitals & 40 & 44.0 & 23 & 25.3 & 19 & 20.9 & 0 & 0.0 & 9 & 9.9 & 0 & 0.0 & 0 & 0.0 & 0 & 0.0 & 91 & 100.0 \\
\hline ALL HOSPITALS & 1271 & 37.4 & 891 & 26.2 & 694 & 20.4 & 33 & 1.0 & 404 & 11.9 & 20 & 0.6 & 86 & 2.5 & 0 & 0.0 & 3399 & 100.0 \\
\hline
\end{tabular}


TABLE 108 (continued)

CONFINEMENTS WITH VAGINAL DELIVERIES BY PERINEAL STATUS AND HOSPITAL, NSW $2000^{*}$

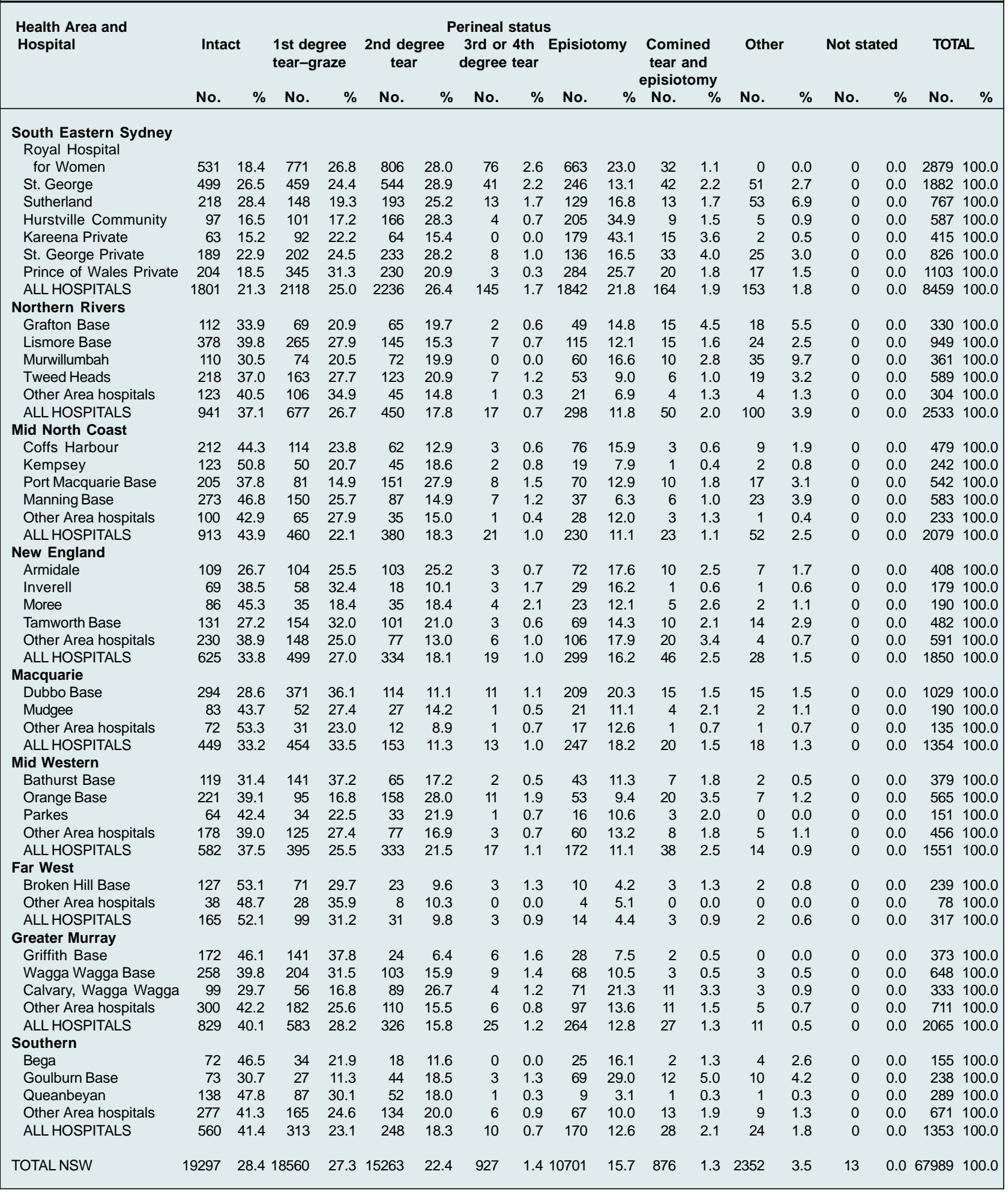

Source: NSW Midwives Data Collection (HOIST). Epidemiology and Surveillance Branch, NSW Department of Health.

\# Hospitals with more than 200 deliveries are identified individually. All hospitals include all public and private hospitals. There were 83 cases of 4 th degree tear reported in 2000. 


\subsection{BIRTHWEIGHT IN SELECTED HOSPITALS}

Table 109 shows the birthweight among live born babies for individual hospitals where the number of reported confinements exceeded 200 in 2000 , totals for all hospitals within each health area and the NSW total.

\section{TABLE 109}

BIRTHS BY BIRTHWEIGHT AND HOSPITAL, NSW 2000"

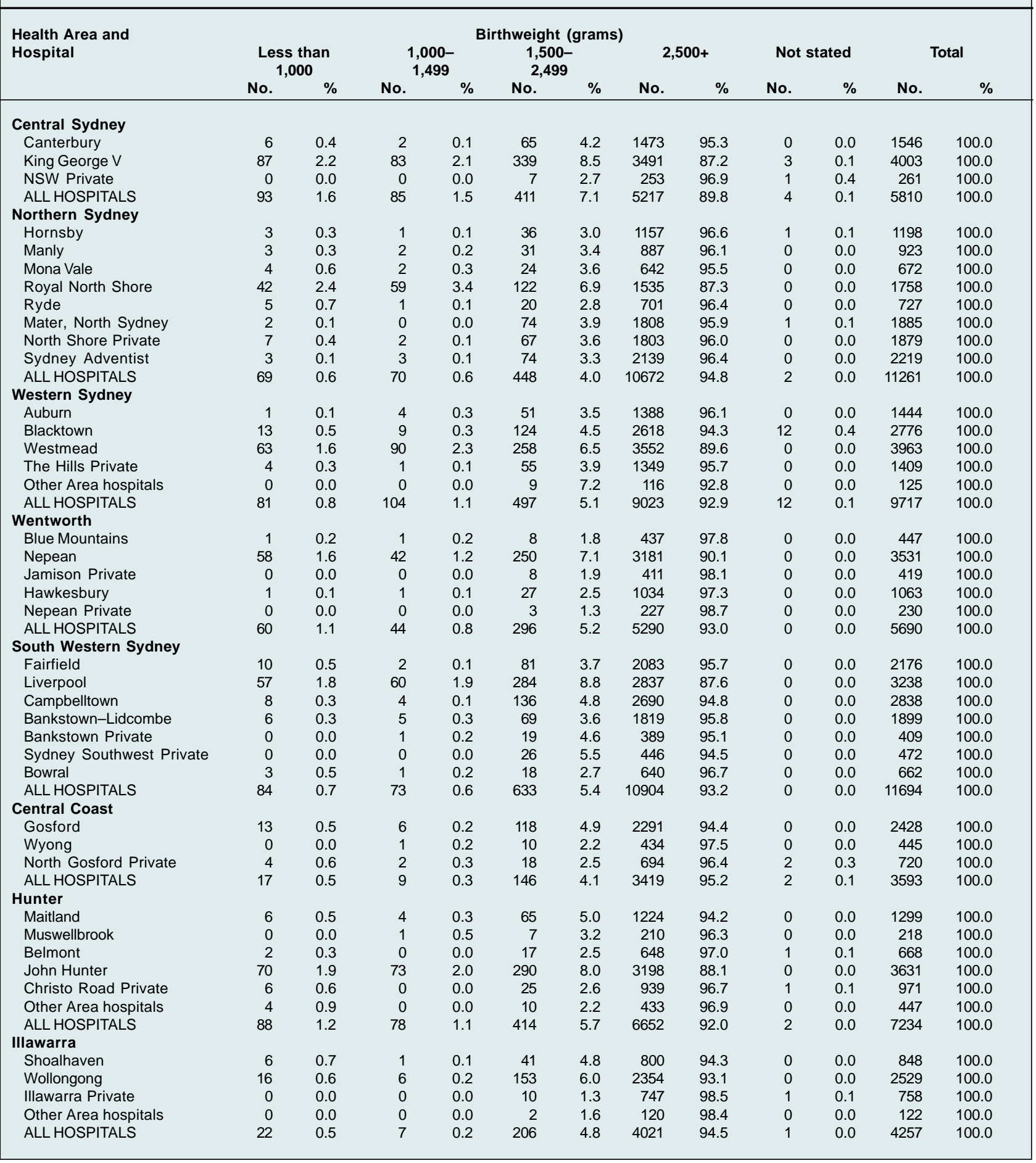




\section{TABLE 109 (continued)}

BIRTHS BY BIRTHWEIGHT AND HOSPITAL, NSW 2000\#

\begin{tabular}{|c|c|c|c|c|c|c|c|c|c|c|c|c|}
\hline \multirow[t]{2}{*}{$\begin{array}{l}\text { Health Area and } \\
\text { Hospital }\end{array}$} & \multicolumn{2}{|c|}{$\begin{array}{c}\text { Less than } \\
1,000\end{array}$} & \multicolumn{2}{|c|}{$\begin{array}{c}1,000- \\
1,499\end{array}$} & \multicolumn{2}{|c|}{$\begin{array}{c}1,500- \\
2,499\end{array}$} & \multicolumn{2}{|c|}{$2,500+$} & \multicolumn{2}{|c|}{ Not stated } & \multicolumn{2}{|c|}{ Total } \\
\hline & No. & $\%$ & No. & $\%$ & No. & $\%$ & No. & $\%$ & No. & $\%$ & No. & $\%$ \\
\hline \multicolumn{13}{|l|}{ South Eastern Sydney } \\
\hline Royal Hospital for Women & 45 & 1.1 & 40 & 1.0 & 242 & 6.1 & 3613 & 91.6 & 6 & 0.2 & 3946 & 100.0 \\
\hline St. George & 14 & 0.6 & 3 & 0.1 & 140 & 5.7 & 2287 & 93.5 & 1 & 0.0 & 2445 & 100.0 \\
\hline Sutherland & 1 & 0.1 & 1 & 0.1 & 31 & 3.2 & 928 & 96.6 & 0 & 0.0 & 961 & 100.0 \\
\hline Hurstville Community & 1 & 0.1 & 1 & 0.1 & 53 & 6.2 & 799 & 93.6 & 0 & 0.0 & 854 & 100.0 \\
\hline Kareena Private & 3 & 0.5 & 0 & 0.0 & 16 & 2.6 & 608 & 97.0 & 0 & 0.0 & 627 & 100.0 \\
\hline St. George Private & 3 & 0.2 & 1 & 0.1 & 39 & 3.0 & 1254 & 96.7 & 0 & 0.0 & 1297 & 100.0 \\
\hline Prince of Wales Private & 1 & 0.1 & 0 & 0.0 & 58 & 3.5 & 1614 & 96.5 & 0 & 0.0 & 1673 & 100.0 \\
\hline ALL HOSPITALS & 68 & 0.6 & 46 & 0.4 & 579 & 4.9 & 11103 & 94.1 & 7 & 0.1 & 11803 & 100.0 \\
\hline \multicolumn{13}{|l|}{ Northern Rivers } \\
\hline Grafton Base & 3 & 0.6 & 2 & 0.4 & 19 & 4.0 & 450 & 94.9 & 0 & 0.0 & 474 & 100.0 \\
\hline Lismore Base & 7 & 0.6 & 4 & 0.3 & 80 & 6.5 & 1139 & 92.6 & 0 & 0.0 & 1230 & 100.0 \\
\hline Murwillumbah & 1 & 0.2 & 0 & 0.0 & 18 & 3.9 & 443 & 95.9 & 0 & 0.0 & 462 & 100.0 \\
\hline Tweed Heads & 5 & 0.7 & 2 & 0.3 & 42 & 5.7 & 685 & 93.2 & 1 & 0.1 & 735 & 100.0 \\
\hline Other Area hospitals & 2 & 0.6 & 1 & 0.3 & 10 & 3.0 & 318 & 95.5 & 2 & 0.6 & 333 & 100.0 \\
\hline ALL HOSPITALS & 18 & 0.6 & 9 & 0.3 & 169 & 5.2 & 3035 & 93.8 & 3 & 0.1 & 3234 & 100.0 \\
\hline \multicolumn{13}{|l|}{ Mid North Coast } \\
\hline Coffs Harbour & 4 & 0.6 & 3 & 0.4 & 43 & 6.0 & 662 & 93.0 & 0 & 0.0 & 712 & 100.0 \\
\hline Kempsey & 1 & 0.3 & 0 & 0.0 & 11 & 3.7 & 284 & 95.9 & 0 & 0.0 & 296 & 100.0 \\
\hline Port Macquarie Base & 3 & 0.4 & 0 & 0.0 & 45 & 6.2 & 675 & 93.2 & 1 & 0.1 & 724 & 100.0 \\
\hline Manning Base & 4 & 0.6 & 2 & 0.3 & 34 & 4.7 & 678 & 94.4 & 0 & 0.0 & 718 & 100.0 \\
\hline Other Area hospitals & 0 & 0.0 & 0 & 0.0 & 11 & 4.0 & 267 & 96.0 & 0 & 0.0 & 278 & 100.0 \\
\hline ALL HOSPITALS & 12 & 0.4 & 5 & 0.2 & 144 & 5.3 & 2566 & 94.1 & 1 & 0.0 & 2728 & 100.0 \\
\hline \multicolumn{13}{|l|}{ New England } \\
\hline Armidale & 3 & 0.7 & 0 & 0.0 & 27 & 5.9 & 430 & 93.5 & 0 & 0.0 & 460 & 100.0 \\
\hline Inverell & 0 & 0.0 & 0 & 0.0 & 8 & 3.4 & 226 & 96.6 & 0 & 0.0 & 234 & 100.0 \\
\hline Moree & 0 & 0.0 & 1 & 0.4 & 9 & 3.7 & 235 & 95.9 & 0 & 0.0 & 245 & 100.0 \\
\hline Tamworth Base & 6 & 0.9 & 1 & 0.2 & 42 & 6.5 & 595 & 92.4 & 0 & 0.0 & 644 & 100.0 \\
\hline Other Area hospitals & 3 & 0.4 & 0 & 0.0 & 23 & 3.1 & 707 & 96.3 & 1 & 0.1 & 734 & 100.0 \\
\hline ALL HOSPITALS & 12 & 0.5 & 2 & 0.1 & 109 & 4.7 & 2193 & 94.6 & 1 & 0.0 & 2317 & 100.0 \\
\hline \multicolumn{13}{|l|}{ Macquarie } \\
\hline Dubbo Base & 2 & 0.2 & 3 & 0.2 & 92 & 7.3 & 1164 & 92.2 & 1 & 0.1 & 1262 & 100.0 \\
\hline Mudgee & 0 & 0.0 & 0 & 0.0 & 8 & 3.3 & 234 & 96.7 & 0 & 0.0 & 242 & 100.0 \\
\hline Other Area hospitals & 0 & 0.0 & 0 & 0.0 & 8 & 5.1 & 148 & 94.3 & 1 & 0.6 & 157 & 100.0 \\
\hline ALL HOSPITALS & 2 & 0.1 & 3 & 0.2 & 108 & 6.5 & 1546 & 93.1 & 2 & 0.1 & 1661 & 100.0 \\
\hline Mid Western & & & & & & & & & & & & \\
\hline Bathurst Base & 1 & 0.2 & 1 & 0.2 & 20 & 3.7 & 512 & 95.9 & 0 & 0.0 & 534 & 100.0 \\
\hline Orange Base & 2 & 0.3 & 4 & 0.5 & 65 & 8.2 & 720 & 91.0 & 0 & 0.0 & 791 & 100.0 \\
\hline Parkes & 1 & 0.5 & 0 & 0.0 & 6 & 2.9 & 199 & 96.6 & 0 & 0.0 & 206 & 100.0 \\
\hline Other Area hospitals & 4 & 0.7 & 1 & 0.2 & 17 & 2.8 & 591 & 96.1 & 2 & 0.3 & 615 & 100.0 \\
\hline ALL HOSPITALS & 8 & 0.4 & 6 & 0.3 & 108 & 5.0 & 2022 & 94.2 & 2 & 0.1 & 2146 & 100.0 \\
\hline Far West & & & & & & & & & & & & \\
\hline Broken Hill Base & 1 & 0.4 & 0 & 0.0 & 14 & 5.0 & 262 & 94.2 & 1 & 0.4 & 278 & 100.0 \\
\hline Other Area hospitals & 3 & 3.6 & 0 & 0.0 & 7 & 8.3 & 74 & 88.1 & 0 & 0.0 & 84 & 100.0 \\
\hline ALL HOSPITALS & 4 & 1.1 & 0 & 0.0 & 21 & 5.8 & 336 & 92.8 & 1 & 0.3 & 362 & 100.0 \\
\hline Greater Murray & & & & & & & & & & & & \\
\hline Griffith Base & 2 & 0.4 & 0 & 0.0 & 16 & 3.3 & 463 & 96.1 & 1 & 0.2 & 482 & 100.0 \\
\hline Wagga Wagga Base & 8 & 1.0 & 1 & 0.1 & 53 & 6.4 & 771 & 92.4 & 1 & 0.1 & 834 & 100.0 \\
\hline Calvary, Wagga Wagga & 2 & 0.5 & 1 & 0.2 & 17 & 3.9 & 420 & 95.5 & 0 & 0.0 & 440 & 100.0 \\
\hline Other Area hospitals & 0 & 0.0 & 2 & 0.2 & 22 & 2.5 & 851 & 97.3 & 0 & 0.0 & 875 & 100.0 \\
\hline ALL HOSPITALS & 12 & 0.5 & 4 & 0.2 & 108 & 4.1 & 2505 & 95.2 & 2 & 0.1 & 2631 & 100.0 \\
\hline Southern & & & & & & & & & & & & \\
\hline Bega & 1 & 0.5 & 0 & 0.0 & 15 & 7.0 & 199 & 92.6 & 0 & 0.0 & 215 & 100.0 \\
\hline Goulburn Base & 0 & 0.0 & 0 & 0.0 & 10 & 3.1 & 308 & 96.6 & 1 & 0.3 & 319 & 100.0 \\
\hline Queanbeyan & 1 & 0.3 & 0 & 0.0 & 11 & 3.3 & 326 & 96.4 & 0 & 0.0 & 338 & 100.0 \\
\hline Other Area hospitals & 1 & 0.1 & 1 & 0.1 & 28 & 3.5 & 772 & 96.0 & 2 & 0.2 & 804 & 100.0 \\
\hline ALL HOSPITALS & 3 & 0.2 & 1 & 0.1 & 64 & 3.8 & 1605 & 95.8 & 3 & 0.2 & 1676 & 100.0 \\
\hline TOTAL NSW & 653 & 0.7 & 546 & 0.6 & 4462 & 5.1 & 82214 & 93.5 & 47 & 0.1 & 87922 & 100.0 \\
\hline
\end{tabular}

Source: NSW Midwives Data Collection (HOIST). Epidemiology and Surveillance Branch, NSW Department of Health.

\# Hospitals with more than 200 total deliveries are identified individually. All hospitals include all public and private hospitals. 


\subsection{GESTATIONAL AGE IN SELECTED HOSPITALS}

Table 110 shows the gestational age among live born babies for individual hospitals where the number of reported confinements exceeded 200 in 2000 , totals for all hospitals within each health area and the NSW total.

\section{TABLE 110}

BIRTHS BY GESTATIONAL AGE AND HOSPITAL, NSW 2000"

\begin{tabular}{|c|c|c|c|c|c|c|c|c|c|c|c|c|}
\hline \multirow{3}{*}{$\begin{array}{l}\text { Health Area and } \\
\text { Hospital }\end{array}$} & \multicolumn{12}{|c|}{ Gestational age (weeks) } \\
\hline & \multicolumn{2}{|c|}{$20-31$} & \multicolumn{2}{|c|}{$32-33$} & \multicolumn{2}{|c|}{$34-36$} & \multicolumn{2}{|c|}{$37+$} & \multicolumn{2}{|c|}{ Not stated } & \multicolumn{2}{|c|}{ TOTAL } \\
\hline & No. & $\%$ & No. & $\%$ & No. & $\%$ & No. & $\%$ & No. & $\%$ & No. & $\%$ \\
\hline \multicolumn{13}{|l|}{ Central Sydney } \\
\hline Canterbury & 7 & 0.5 & 2 & 0.1 & 69 & 4.5 & 1468 & 95.0 & 0 & 0.0 & 1546 & 100.0 \\
\hline King George V & 183 & 4.6 & 124 & 3.1 & 233 & 5.8 & 3463 & 86.5 & 0 & 0.0 & 4003 & 100.0 \\
\hline NSW Private & 0 & 0.0 & 1 & 0.4 & 9 & 3.4 & 251 & 96.2 & 0 & 0.0 & 261 & 100.0 \\
\hline ALL HOSPITALS & 190 & 3.3 & 127 & 2.2 & 311 & 5.4 & 5182 & 89.2 & 0 & 0.0 & 5810 & 100.0 \\
\hline \multicolumn{13}{|l|}{ Northern Sydney } \\
\hline Hornsby & 5 & 0.4 & 1 & 0.1 & 45 & 3.8 & 1147 & 95.7 & 0 & 0.0 & 1198 & 100.0 \\
\hline Manly & 4 & 0.4 & 0 & 0.0 & 57 & 6.2 & 862 & 93.4 & 0 & 0.0 & 923 & 100.0 \\
\hline Mona Vale & 6 & 0.9 & 3 & 0.4 & 23 & 3.4 & 640 & 95.2 & 0 & 0.0 & 672 & 100.0 \\
\hline Royal North Shore & 108 & 6.1 & 57 & 3.2 & 76 & 4.3 & 1517 & 86.3 & 0 & 0.0 & 1758 & 100.0 \\
\hline Ryde & 5 & 0.7 & 1 & 0.1 & 16 & 2.2 & 705 & 97.0 & 0 & 0.0 & 727 & 100.0 \\
\hline Mater, North Sydney & 4 & 0.2 & 4 & 0.2 & 89 & 4.7 & 1788 & 94.9 & 0 & 0.0 & 1885 & 100.0 \\
\hline North Shore Private & 7 & 0.4 & 7 & 0.4 & 88 & 4.7 & 1777 & 94.6 & 0 & 0.0 & 1879 & 100.0 \\
\hline Sydney Adventist & 4 & 0.2 & 10 & 0.5 & 105 & 4.7 & 2100 & 94.6 & 0 & 0.0 & 2219 & 100.0 \\
\hline ALL HOSPITALS & 143 & 1.3 & 83 & 0.7 & 499 & 4.4 & 10536 & 93.6 & 0 & 0.0 & 11261 & 100.0 \\
\hline \multicolumn{13}{|l|}{ Western Sydney } \\
\hline Auburn & 5 & 0.3 & 8 & 0.6 & 39 & 2.7 & 1392 & 96.4 & 0 & 0.0 & 1444 & 100.0 \\
\hline Blacktown & 24 & 0.9 & 11 & 0.4 & 124 & 4.5 & 2612 & 94.1 & 5 & 0.2 & 2776 & 100.0 \\
\hline Westmead & 153 & 3.9 & 71 & 1.8 & 178 & 4.5 & 3561 & 89.9 & 0 & 0.0 & 3963 & 100.0 \\
\hline The Hills Private & 5 & 0.4 & 3 & 0.2 & 74 & 5.3 & 1327 & 94.2 & 0 & 0.0 & 1409 & 100.0 \\
\hline Other Area hospitals & 0 & 0.0 & 1 & 0.8 & 5 & 4.0 & 119 & 95.2 & 0 & 0.0 & 125 & 100.0 \\
\hline ALL HOSPITALS & 187 & 1.9 & 94 & 1.0 & 420 & 4.3 & 9011 & 92.7 & 5 & 0.1 & 9717 & 100.0 \\
\hline \multicolumn{13}{|l|}{ Wentworth } \\
\hline Blue Mountains & 2 & 0.4 & 0 & 0.0 & 12 & 2.7 & 433 & 96.9 & 0 & 0.0 & 447 & 100.0 \\
\hline Nepean & 105 & 3.0 & 68 & 1.9 & 209 & 5.9 & 3149 & 89.2 & 0 & 0.0 & 3531 & 100.0 \\
\hline Jamison Private & 1 & 0.2 & 1 & 0.2 & 9 & 2.1 & 408 & 97.4 & 0 & 0.0 & 419 & 100.0 \\
\hline Hawkesbury & 2 & 0.2 & 0 & 0.0 & 42 & 4.0 & 1019 & 95.9 & 0 & 0.0 & 1063 & 100.0 \\
\hline Nepean Private & 0 & 0.0 & 0 & 0.0 & 5 & 2.2 & 225 & 97.8 & 0 & 0.0 & 230 & 100.0 \\
\hline ALL HOSPITALS & 110 & 1.9 & 69 & 1.2 & 277 & 4.9 & 5234 & 92.0 & 0 & 0.0 & 5690 & 100.0 \\
\hline \multicolumn{13}{|l|}{ South Western Sydney } \\
\hline Fairfield & 12 & 0.6 & 6 & 0.3 & 85 & 3.9 & 2073 & 95.3 & 0 & 0.0 & 2176 & 100.0 \\
\hline Liverpool & 117 & 3.6 & 99 & 3.1 & 194 & 6.0 & 2828 & 87.3 & 0 & 0.0 & 3238 & 100.0 \\
\hline Campbelltown & 16 & 0.6 & 6 & 0.2 & 134 & 4.7 & 2682 & 94.5 & 0 & 0.0 & 2838 & 100.0 \\
\hline Bankstown-Lidcombe & 7 & 0.4 & 6 & 0.3 & 74 & 3.9 & 1812 & 95.4 & 0 & 0.0 & 1899 & 100.0 \\
\hline Bankstown Private & 1 & 0.2 & 2 & 0.5 & 19 & 4.6 & 387 & 94.6 & 0 & 0.0 & 409 & 100.0 \\
\hline Sydney Southwest Private & 0 & 0.0 & 0 & 0.0 & 35 & 7.4 & 437 & 92.6 & 0 & 0.0 & 472 & 100.0 \\
\hline Bowral & 4 & 0.6 & 0 & 0.0 & 16 & 2.4 & 642 & 97.0 & 0 & 0.0 & 662 & 100.0 \\
\hline ALL HOSPITALS & 157 & 1.3 & 119 & 1.0 & 557 & 4.8 & 10861 & 92.9 & 0 & 0.0 & 11694 & 100.0 \\
\hline Central Coast & & & & & & & & & & & & \\
\hline Gosford & 20 & 0.8 & 17 & 0.7 & 161 & 6.6 & 2230 & 91.8 & 0 & 0.0 & 2428 & 100.0 \\
\hline Wyong & 1 & 0.2 & 3 & 0.7 & 12 & 2.7 & 429 & 96.4 & 0 & 0.0 & 445 & 100.0 \\
\hline North Gosford Private & 7 & 1.0 & 0 & 0.0 & 32 & 4.4 & 681 & 94.6 & 0 & 0.0 & 720 & 100.0 \\
\hline ALL HOSPITALS & 28 & 0.8 & 20 & 0.6 & 205 & 5.7 & 3340 & 93.0 & 0 & 0.0 & 3593 & 100.0 \\
\hline Hunter & & & & & & & & & & & & \\
\hline Maitland & 8 & 0.6 & 3 & 0.2 & 69 & 5.3 & 1219 & 93.8 & 0 & 0.0 & 1299 & 100.0 \\
\hline Muswellbrook & 1 & 0.5 & 0 & 0.0 & 6 & 2.8 & 211 & 96.8 & 0 & 0.0 & 218 & 100.0 \\
\hline Belmont & 3 & 0.4 & 0 & 0.0 & 27 & 4.0 & 638 & 95.5 & 0 & 0.0 & 668 & 100.0 \\
\hline John Hunter & 152 & 4.2 & 85 & 2.3 & 233 & 6.4 & 3161 & 87.1 & 0 & 0.0 & 3631 & 100.0 \\
\hline Christo Road Private & 7 & 0.7 & 2 & 0.2 & 64 & 6.6 & 898 & 92.5 & 0 & 0.0 & 971 & 100.0 \\
\hline Other Area hospitals & 4 & 0.9 & 1 & 0.2 & 16 & 3.6 & 426 & 95.3 & 0 & 0.0 & 447 & 100.0 \\
\hline ALL HOSPITALS & 175 & 2.4 & 91 & 1.3 & 415 & 5.7 & 6553 & 90.6 & 0 & 0.0 & 7234 & 100.0 \\
\hline Illawarra & & & & & & & & & & & & \\
\hline Shoalhaven & 8 & 0.9 & 3 & 0.4 & 33 & 3.9 & 804 & 94.8 & 0 & 0.0 & 848 & 100.0 \\
\hline Wollongong & 26 & 1.0 & 29 & 1.1 & 147 & 5.8 & 2327 & 92.0 & 0 & 0.0 & 2529 & 100.0 \\
\hline Illawarra Private & 1 & 0.1 & 0 & 0.0 & 7 & 0.9 & 750 & 98.9 & 0 & 0.0 & 758 & 100.0 \\
\hline Other Area hospitals & 0 & 0.0 & 0 & 0.0 & 1 & 0.8 & 121 & 99.2 & 0 & 0.0 & 122 & 100.0 \\
\hline ALL HOSPITALS & 35 & 0.8 & 32 & 0.8 & 188 & 4.4 & 4002 & 94.0 & 0 & 0.0 & 4257 & 100.0 \\
\hline
\end{tabular}




\section{TABLE 110 (continued)}

BIRTHS BY GESTATIONAL AGE AND HOSPITAL, NSW 2000\#

\begin{tabular}{|c|c|c|c|c|c|c|c|c|c|c|c|c|}
\hline \multirow{3}{*}{$\begin{array}{l}\text { Health Area and } \\
\text { Hospital }\end{array}$} & \multicolumn{12}{|c|}{ Gestational age (weeks) } \\
\hline & \multicolumn{2}{|c|}{ 20-31 } & \multicolumn{2}{|c|}{$32-33$} & \multicolumn{2}{|c|}{$34-36$} & \multicolumn{2}{|c|}{$37+$} & \multicolumn{2}{|c|}{ Not stated } & \multicolumn{2}{|c|}{ TOTAL } \\
\hline & No. & $\%$ & No. & $\%$ & No. & $\%$ & No. & $\%$ & No. & $\%$ & No. & $\%$ \\
\hline \multicolumn{13}{|l|}{ South Eastern Sydney } \\
\hline Royal Hospital for Women & 105 & 2.7 & 57 & 1.4 & 213 & 5.4 & 3571 & 90.5 & 0 & 0.0 & 3946 & 100.0 \\
\hline St. George & 18 & 0.7 & 22 & 0.9 & 172 & 7.0 & 2233 & 91.3 & 0 & 0.0 & 2445 & 100.0 \\
\hline Sutherland & 2 & 0.2 & 2 & 0.2 & 47 & 4.9 & 910 & 94.7 & 0 & 0.0 & 961 & 100.0 \\
\hline Hurstville Community & 1 & 0.1 & 5 & 0.6 & 48 & 5.6 & 800 & 93.7 & 0 & 0.0 & 854 & 100.0 \\
\hline Kareena Private & 4 & 0.6 & 2 & 0.3 & 34 & 5.4 & 587 & 93.6 & 0 & 0.0 & 627 & 100.0 \\
\hline St. George Private & 4 & 0.3 & 5 & 0.4 & 71 & 5.5 & 1217 & 93.8 & 0 & 0.0 & 1297 & 100.0 \\
\hline Prince of Wales Private & 3 & 0.2 & 5 & 0.3 & 66 & 3.9 & 1599 & 95.6 & 0 & 0.0 & 1673 & 100.0 \\
\hline ALL HOSPITALS & 137 & 1.2 & 98 & 0.8 & 651 & 5.5 & 10917 & 92.5 & 0 & 0.0 & 11803 & 100.0 \\
\hline \multicolumn{13}{|l|}{ Northern Rivers } \\
\hline Grafton Base & 5 & 1.1 & 0 & 0.0 & 21 & 4.4 & 448 & 94.5 & 0 & 0.0 & 474 & 100.0 \\
\hline Lismore Base & 14 & 1.1 & 18 & 1.5 & 74 & 6.0 & 1124 & 91.4 & 0 & 0.0 & 1230 & 100.0 \\
\hline Murwillumbah & 2 & 0.4 & 0 & 0.0 & 17 & 3.7 & 443 & 95.9 & 0 & 0.0 & 462 & 100.0 \\
\hline Tweed Heads & 10 & 1.4 & 3 & 0.4 & 41 & 5.6 & 681 & 92.7 & 0 & 0.0 & 735 & 100.0 \\
\hline Other Area hospitals & 4 & 1.2 & 0 & 0.0 & 9 & 2.7 & 320 & 96.1 & 0 & 0.0 & 333 & 100.0 \\
\hline ALL HOSPITALS & 35 & 1.1 & 21 & 0.6 & 162 & 5.0 & 3016 & 93.3 & 0 & 0.0 & 3234 & 100.0 \\
\hline \multicolumn{13}{|l|}{ Mid North Coast } \\
\hline Coffs Harbour & 7 & 1.0 & 3 & 0.4 & 41 & 5.8 & 661 & 92.8 & 0 & 0.0 & 712 & 100.0 \\
\hline Kempsey & 0 & 0.0 & 0 & 0.0 & 10 & 3.4 & 286 & 96.6 & 0 & 0.0 & 296 & 100.0 \\
\hline Port Macquarie Base & 4 & 0.6 & 1 & 0.1 & 53 & 7.3 & 666 & 92.0 & 0 & 0.0 & 724 & 100.0 \\
\hline Manning Base & 8 & 1.1 & 5 & 0.7 & 27 & 3.8 & 678 & 94.4 & 0 & 0.0 & 718 & 100.0 \\
\hline Other Area hospitals & 0 & 0.0 & 1 & 0.4 & 6 & 2.2 & 271 & 97.5 & 0 & 0.0 & 278 & 100.0 \\
\hline ALL HOSPITALS & 19 & 0.7 & 10 & 0.4 & 137 & 5.0 & 2562 & 93.9 & 0 & 0.0 & 2728 & 100.0 \\
\hline \multicolumn{13}{|l|}{ New England } \\
\hline Armidale & 3 & 0.7 & 2 & 0.4 & 18 & 3.9 & 437 & 95.0 & 0 & 0.0 & 460 & 100.0 \\
\hline Inverell & 0 & 0.0 & 1 & 0.4 & 6 & 2.6 & 227 & 97.0 & 0 & 0.0 & 234 & 100.0 \\
\hline Moree & 1 & 0.4 & 0 & 0.0 & 8 & 3.3 & 236 & 96.3 & 0 & 0.0 & 245 & 100.0 \\
\hline Tamworth Base & 9 & 1.4 & 2 & 0.3 & 53 & 8.2 & 580 & 90.1 & 0 & 0.0 & 644 & 100.0 \\
\hline Other Area hospitals & 3 & 0.4 & 1 & 0.1 & 14 & 1.9 & 716 & 97.5 & 0 & 0.0 & 734 & 100.0 \\
\hline ALL HOSPITALS & 16 & 0.7 & 6 & 0.3 & 99 & 4.3 & 2196 & 94.8 & 0 & 0.0 & 2317 & 100.0 \\
\hline Macquarie & & & & & & & & & & & & \\
\hline Dubbo Base & 6 & 0.5 & 6 & 0.5 & 69 & 5.5 & 1181 & 93.6 & 0 & 0.0 & 1262 & 100.0 \\
\hline Mudgee & 0 & 0.0 & 0 & 0.0 & 4 & 1.7 & 238 & 98.3 & 0 & 0.0 & 242 & 100.0 \\
\hline Other Area hospitals & 0 & 0.0 & 0 & 0.0 & 6 & 3.8 & 151 & 96.2 & 0 & 0.0 & 157 & 100.0 \\
\hline ALL HOSPITALS & 6 & 0.4 & 6 & 0.4 & 79 & 4.8 & 1570 & 94.5 & 0 & 0.0 & 1661 & 100.0 \\
\hline Mid Western & & & & & & & & & & & & \\
\hline Bathurst Base & 3 & 0.6 & 2 & 0.4 & 26 & 4.9 & 503 & 94.2 & 0 & 0.0 & 534 & 100.0 \\
\hline Orange Base & 8 & 1.0 & 6 & 0.8 & 74 & 9.4 & 703 & 88.9 & 0 & 0.0 & 791 & 100.0 \\
\hline Parkes & 1 & 0.5 & 0 & 0.0 & 6 & 2.9 & 199 & 96.6 & 0 & 0.0 & 206 & 100.0 \\
\hline Other Area hospitals & 5 & 0.8 & 2 & 0.3 & 16 & 2.6 & 592 & 96.3 & 0 & 0.0 & 615 & 100.0 \\
\hline ALL HOSPITALS & 17 & 0.8 & 10 & 0.5 & 122 & 5.7 & 1997 & 93.1 & 0 & 0.0 & 2146 & 100.0 \\
\hline Far West & & & & & & & & & & & & \\
\hline Broken Hill Base & 2 & 0.7 & 1 & 0.4 & 7 & 2.5 & 268 & 96.4 & 0 & 0.0 & 278 & 100.0 \\
\hline Other Area hospitals & 3 & 3.6 & 0 & 0.0 & 6 & 7.1 & 75 & 89.3 & 0 & 0.0 & 84 & 100.0 \\
\hline ALL HOSPITALS & 5 & 1.4 & 1 & 0.3 & 13 & 3.6 & 343 & 94.8 & 0 & 0.0 & 362 & 100.0 \\
\hline Greater Murray & & & & & & & & & & & & \\
\hline Griffith Base & 3 & 0.6 & 4 & 0.8 & 21 & 4.4 & 454 & 94.2 & 0 & 0.0 & 482 & 100.0 \\
\hline Wagga Wagga Base & 11 & 1.3 & 8 & 1.0 & 47 & 5.6 & 768 & 92.1 & 0 & 0.0 & 834 & 100.0 \\
\hline Calvary, Wagga Wagga & 3 & 0.7 & 4 & 0.9 & 25 & 5.7 & 408 & 92.7 & 0 & 0.0 & 440 & 100.0 \\
\hline Other Area hospitals & 3 & 0.3 & 1 & 0.1 & 16 & 1.8 & 854 & 97.6 & 1 & 0.1 & 875 & 100.0 \\
\hline ALL HOSPITALS & 20 & 0.8 & 17 & 0.6 & 109 & 4.1 & 2484 & 94.4 & 1 & 0.0 & 2631 & 100.0 \\
\hline Southern & & & & & & & & & & & & \\
\hline Bega & 2 & 0.9 & 2 & 0.9 & 14 & 6.5 & 197 & 91.6 & 0 & 0.0 & 215 & 100.0 \\
\hline Goulburn Base & 1 & 0.3 & 0 & 0.0 & 13 & 4.1 & 305 & 95.6 & 0 & 0.0 & 319 & 100.0 \\
\hline Queanbeyan & 1 & 0.3 & 0 & 0.0 & 11 & 3.3 & 326 & 96.4 & 0 & 0.0 & 338 & 100.0 \\
\hline Other Area hospitals & 2 & 0.2 & 1 & 0.1 & 24 & 3.0 & 777 & 96.6 & 0 & 0.0 & 804 & 100.0 \\
\hline ALL HOSPITALS & 6 & 0.4 & 3 & 0.2 & 62 & 3.7 & 1605 & 95.8 & 0 & 0.0 & 1676 & 100.0 \\
\hline TOTAL NSW & 1286 & 1.5 & 807 & 0.9 & 4307 & 4.98 & 1516 & 92.7 & 6 & 0.0 & 87922 & 100.0 \\
\hline
\end{tabular}

Source: NSW Midwives Data Collection (HOIST). Epidemiology and Surveillance Branch, NSW Department of Health.

\# Hospitals with more than 200 deliveries are identified individually. All hospitals include all public and private hospitals. 


\subsection{BABY DISCHARGE STATUS IN SELECTED HOSPITALS}

Table 111 shows the discharge status of babies born in hospitals where the number of reported confinements exceeded 200 in 2000, totals for all hospitals within each health area and the NSW total.

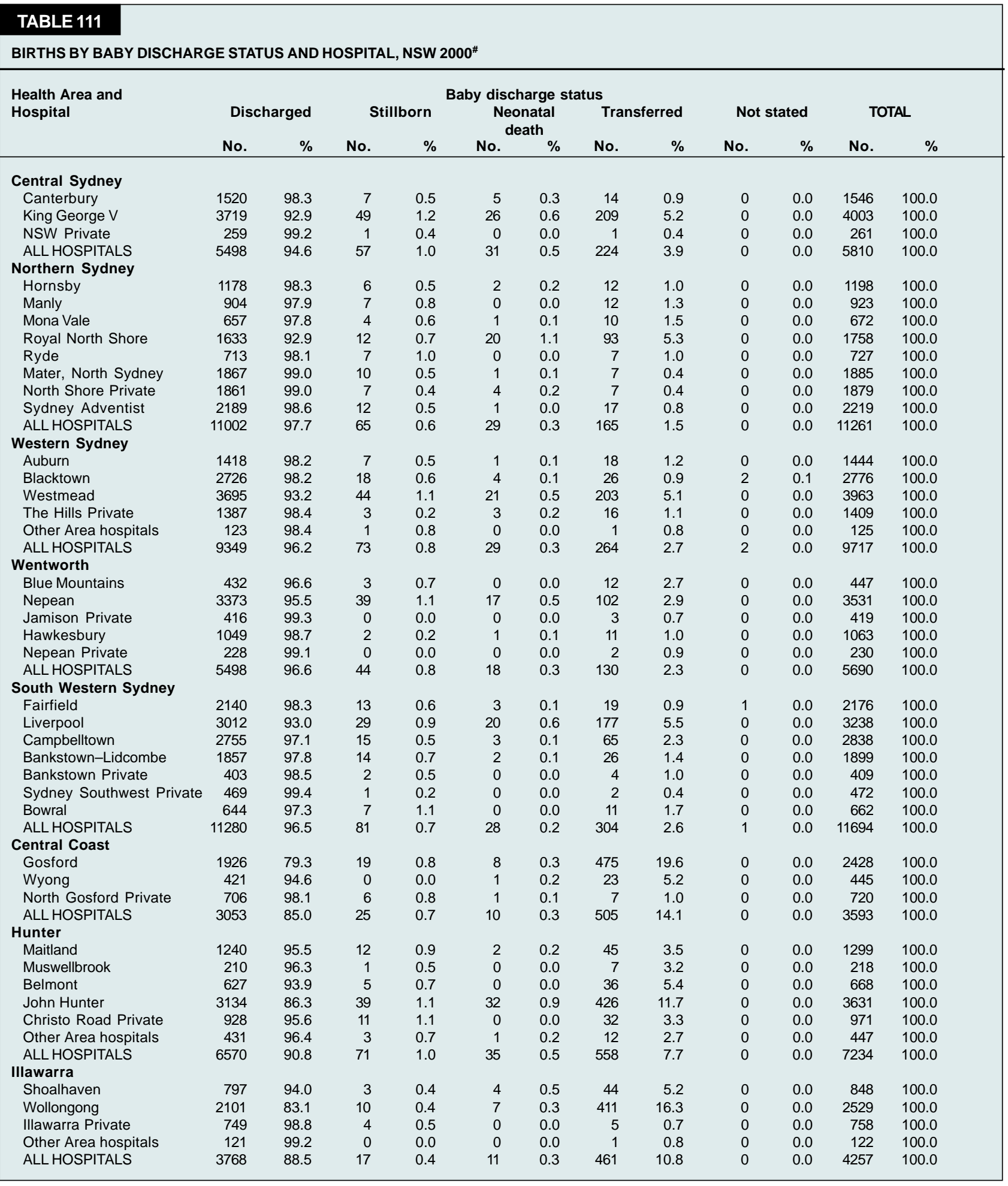




\section{TABLE 111 (continued)}

BIRTHS BY BABY DISCHARGE STATUS AND HOSPITAL, NSW 2000\#

\begin{tabular}{|c|c|c|c|c|c|c|c|c|c|c|c|c|}
\hline \multirow{3}{*}{$\begin{array}{l}\text { Health Area and } \\
\text { Hospital }\end{array}$} & \multicolumn{12}{|c|}{ Baby discharge status } \\
\hline & \multicolumn{2}{|c|}{ Discharged } & \multicolumn{2}{|c|}{ Stillborn } & \multicolumn{2}{|c|}{$\begin{array}{c}\text { Neonatal } \\
\text { death }\end{array}$} & \multicolumn{2}{|c|}{ Transferred } & \multicolumn{2}{|c|}{ Not stated } & \multicolumn{2}{|c|}{ TOTAL } \\
\hline & No. & $\%$ & No. & $\%$ & No. & $\%$ & No. & $\%$ & No. & $\%$ & No. & $\%$ \\
\hline \multicolumn{13}{|l|}{ South Eastern Sydney } \\
\hline Royal Hospital for Women & 3802 & 96.4 & 27 & 0.7 & 18 & 0.5 & 99 & 2.5 & 0 & 0.0 & 3946 & 100.0 \\
\hline St. George & 2404 & 98.3 & 16 & 0.7 & 7 & 0.3 & 18 & 0.7 & 0 & 0.0 & 2445 & 100.0 \\
\hline Sutherland & 949 & 98.8 & 2 & 0.2 & 1 & 0.1 & 9 & 0.9 & 0 & 0.0 & 961 & 100.0 \\
\hline Hurstville Community & 838 & 98.1 & 6 & 0.7 & 0 & 0.0 & 10 & 1.2 & 0 & 0.0 & 854 & 100.0 \\
\hline Kareena Private & 620 & 98.9 & 4 & 0.6 & 0 & 0.0 & 3 & 0.5 & 0 & 0.0 & 627 & 100.0 \\
\hline St. George Private & 1283 & 98.9 & 5 & 0.4 & 1 & 0.1 & 8 & 0.6 & 0 & 0.0 & 1297 & 100.0 \\
\hline Prince of Wales Private & 1655 & 98.9 & 1 & 0.1 & 1 & 0.1 & 16 & 1.0 & 0 & 0.0 & 1673 & 100.0 \\
\hline ALL HOSPITALS & 11551 & 97.9 & 61 & 0.5 & 28 & 0.2 & 163 & 1.4 & 0 & 0.0 & 11803 & 100.0 \\
\hline \multicolumn{13}{|l|}{ Northern Rivers } \\
\hline Grafton Base & 452 & 95.4 & 4 & 0.8 & 0 & 0.0 & 18 & 3.8 & 0 & 0.0 & 474 & 100.0 \\
\hline Lismore Base & 953 & 77.5 & 11 & 0.9 & 4 & 0.3 & 262 & 21.3 & 0 & 0.0 & 1230 & 100.0 \\
\hline Murwillumbah & 446 & 96.5 & 2 & 0.4 & 0 & 0.0 & 14 & 3.0 & 0 & 0.0 & 462 & 100.0 \\
\hline Tweed Heads & 721 & 98.1 & 5 & 0.7 & 1 & 0.1 & 8 & 1.1 & 0 & 0.0 & 735 & 100.0 \\
\hline Other Area hospitals & 314 & 94.3 & 2 & 0.6 & 1 & 0.3 & 16 & 4.8 & 0 & 0.0 & 333 & 100.0 \\
\hline ALL HOSPITALS & 2886 & 89.2 & 24 & 0.7 & 6 & 0.2 & 318 & 9.8 & 0 & 0.0 & 3234 & 100.0 \\
\hline \multicolumn{13}{|l|}{ Mid North Coast } \\
\hline Coffs Harbour & 648 & 91.0 & 2 & 0.3 & 4 & 0.6 & 58 & 8.1 & 0 & 0.0 & 712 & 100.0 \\
\hline Kempsey & 287 & 97.0 & 1 & 0.3 & 0 & 0.0 & 8 & 2.7 & 0 & 0.0 & 296 & 100.0 \\
\hline Port Macquarie Base & 665 & 91.9 & 5 & 0.7 & 1 & 0.1 & 53 & 7.3 & 0 & 0.0 & 724 & 100.0 \\
\hline Manning Base & 670 & 93.3 & 8 & 1.1 & 1 & 0.1 & 39 & 5.4 & 0 & 0.0 & 718 & 100.0 \\
\hline Other Area hospitals & 265 & 95.3 & 1 & 0.4 & 0 & 0.0 & 12 & 4.3 & 0 & 0.0 & 278 & 100.0 \\
\hline ALL HOSPITALS & 2535 & 92.9 & 17 & 0.6 & 6 & 0.2 & 170 & 6.2 & 0 & 0.0 & 2728 & 100.0 \\
\hline \multicolumn{13}{|l|}{ New England } \\
\hline Armidale & 424 & 92.2 & 4 & 0.9 & 0 & 0.0 & 32 & 7.0 & 0 & 0.0 & 460 & 100.0 \\
\hline Inverell & 220 & 94.0 & 1 & 0.4 & 0 & 0.0 & 13 & 5.6 & 0 & 0.0 & 234 & 100.0 \\
\hline Moree & 237 & 96.7 & 1 & 0.4 & 0 & 0.0 & 7 & 2.9 & 0 & 0.0 & 245 & 100.0 \\
\hline Tamworth Base & 559 & 86.8 & 5 & 0.8 & 0 & 0.0 & 80 & 12.4 & 0 & 0.0 & 644 & 100.0 \\
\hline Other Area hospitals & 695 & 94.7 & 1 & 0.1 & 2 & 0.3 & 35 & 4.8 & 1 & 0.1 & 734 & 100.0 \\
\hline ALL HOSPITALS & 2135 & 92.1 & 12 & 0.5 & 2 & 0.1 & 167 & 7.2 & 1 & 0.0 & 2317 & 100.0 \\
\hline Macquarie & & & & & & & & & & & & \\
\hline Dubbo Base & 836 & 66.2 & 8 & 0.6 & 2 & 0.2 & 416 & 33.0 & 0 & 0.0 & 1262 & 100.0 \\
\hline Mudgee & 232 & 95.9 & 0 & 0.0 & 0 & 0.0 & 10 & 4.1 & 0 & 0.0 & 242 & 100.0 \\
\hline Other Area hospitals & 142 & 90.4 & 0 & 0.0 & 0 & 0.0 & 15 & 9.6 & 0 & 0.0 & 157 & 100.0 \\
\hline ALL HOSPITALS & 1210 & 72.8 & 8 & 0.5 & 2 & 0.1 & 441 & 26.6 & 0 & 0.0 & 1661 & 100.0 \\
\hline Mid Western & & & & & & & & & & & & \\
\hline Bathurst Base & 453 & 84.8 & 2 & 0.4 & 0 & 0.0 & 79 & 14.8 & 0 & 0.0 & 534 & 100.0 \\
\hline Orange Base & 638 & 80.7 & 4 & 0.5 & 0 & 0.0 & 149 & 18.8 & 0 & 0.0 & 791 & 100.0 \\
\hline Parkes & 194 & 94.2 & 1 & 0.5 & 0 & 0.0 & 11 & 5.3 & 0 & 0.0 & 206 & 100.0 \\
\hline Other Area hospitals & 590 & 95.9 & 4 & 0.7 & 1 & 0.2 & 20 & 3.3 & 0 & 0.0 & 615 & 100.0 \\
\hline ALL HOSPITALS & 1875 & 87.4 & 11 & 0.5 & 1 & 0.0 & 259 & 12.1 & 0 & 0.0 & 2146 & 100.0 \\
\hline Far West & & & & & & & & & & & & \\
\hline Broken Hill Base & 274 & 98.6 & 2 & 0.7 & 0 & 0.0 & 2 & 0.7 & 0 & 0.0 & 278 & 100.0 \\
\hline Other Area hospitals & 72 & 85.7 & 1 & 1.2 & 2 & 2.4 & 9 & 10.7 & 0 & 0.0 & 84 & 100.0 \\
\hline ALL HOSPITALS & 346 & 95.6 & 3 & 0.8 & 2 & 0.6 & 11 & 3.0 & 0 & 0.0 & 362 & 100.0 \\
\hline Greater Murray & & & & & & & & & & & & \\
\hline Griffith Base & 463 & 96.1 & 5 & 1.0 & 1 & 0.2 & 13 & 2.7 & 0 & 0.0 & 482 & 100.0 \\
\hline Wagga Wagga Base & 743 & 89.1 & 7 & 0.8 & 3 & 0.4 & 81 & 9.7 & 0 & 0.0 & 834 & 100.0 \\
\hline Calvary, Wagga Wagga & 430 & 97.7 & 5 & 1.1 & 1 & 0.2 & 4 & 0.9 & 0 & 0.0 & 440 & 100.0 \\
\hline Other Area hospitals & 830 & 94.9 & 1 & 0.1 & 0 & 0.0 & 44 & 5.0 & 0 & 0.0 & 875 & 100.0 \\
\hline ALL HOSPITALS & 2466 & 93.7 & 18 & 0.7 & 5 & 0.2 & 142 & 5.4 & 0 & 0.0 & 2631 & 100.0 \\
\hline Southern & & & & & & & & & & & & \\
\hline Bega & 183 & 85.1 & 2 & 0.9 & 0 & 0.0 & 30 & 14.0 & 0 & 0.0 & 215 & 100.0 \\
\hline Goulburn Base & 308 & 96.6 & 1 & 0.3 & 0 & 0.0 & 10 & 3.1 & 0 & 0.0 & 319 & 100.0 \\
\hline Queanbeyan & 330 & 97.6 & 2 & 0.6 & 0 & 0.0 & 6 & 1.8 & 0 & 0.0 & 338 & 100.0 \\
\hline Other Area hospitals & 783 & 97.4 & 3 & 0.4 & 0 & 0.0 & 18 & 2.2 & 0 & 0.0 & 804 & 100.0 \\
\hline ALL HOSPITALS & 1604 & 95.7 & 8 & 0.5 & 0 & 0.0 & 64 & 3.8 & 0 & 0.0 & 1676 & 100.0 \\
\hline TOTAL NSW & 82731 & 94.1 & 595 & 0.7 & 243 & 0.3 & 4349 & 4.9 & 4 & 0.0 & 87922 & 100.0 \\
\hline
\end{tabular}

Source: NSW Midwives Data Collection (HOIST). Epidemiology and Surveillance Branch, NSW Department of Health.

\# Hospitals with more than 200 deliveries are identified individually. All hospitals include all public and private hospitals. 


\subsection{POSTNATAL LENGTH OF STAY IN SELECTED HOSPITALS}

Table 112 shows the mother's postnatal length of stay in the hospital of birth for hospitals where the number of reported confinements exceeded 200 in 1999, totals for all hospitals within each health area and the NSW total.

\section{TABLE 112}

AVERAGE MATERNAL POSTNATAL LENGTH OF STAY IN HOSPITAL OF BIRTH, NSW 1995-1999\#

\begin{tabular}{|c|c|c|c|c|c|c|c|c|c|c|c|}
\hline \multirow{4}{*}{$\begin{array}{l}\begin{array}{l}\text { Health Area and } \\
\text { Hospital }\end{array} \\
\begin{array}{l}\text { Central Sydney } \\
\text { Canterbury }\end{array}\end{array}$} & \multicolumn{5}{|c|}{ Average postnatal length of stay (days) } & \multirow{2}{*}{$\begin{array}{l}\text { Health Area and } \\
\text { Hospital }\end{array}$} & \multicolumn{5}{|c|}{ rage postnatal length of stay (days) } \\
\hline & 1995 & 1996 & 1997 & 1998 & 1999 & & 1995 & 1996 & 1997 & 1998 & 1999 \\
\hline & & & & & & \multicolumn{6}{|l|}{ South Eastern Sydney } \\
\hline & 3.5 & 3.1 & - & 2.8 & 2.9 & Royal Hospital for Women & 4.6 & 4.2 & 4.1 & 3.8 & 3.6 \\
\hline King George V & 4.1 & 3.7 & 3.9 & 3.9 & 4.0 & St. George & 4.1 & 3.8 & 3.9 & 3.6 & 3.5 \\
\hline NSW Private & 5.8 & 5.4 & 5.4 & 4.6 & 5.0 & Sutherland & 4.5 & 4.1 & 3.8 & 3.8 & 3.6 \\
\hline ALL HOSPITALS & 4.1 & 3.8 & 4.0 & 3.9 & 3.8 & Hurstville Community & 6.2 & 6.5 & 6.6 & 6.4 & 5.5 \\
\hline \multicolumn{6}{|l|}{ Northern Sydney } & Kareena Private & 6.3 & 6.4 & 6.3 & 5.9 & 5.9 \\
\hline Hornsby & 4.4 & 4.1 & 3.7 & 3.8 & 3.7 & St. George Private & 8.0 & 6.2 & 6.2 & 5.5 & 5.3 \\
\hline Manly & 4.3 & 3.9 & 3.8 & 3.7 & 3.8 & Prince of Wales Private & - & - & 6.3 & 5.6 & 5.2 \\
\hline Mona Vale & 4.3 & 4.2 & 3.9 & 3.8 & 3.7 & Other Area hospitals & 6.0 & 5.8 & 5.8 & 5.6 & - \\
\hline Royal North Shore & 4.3 & 4.3 & 3.9 & 4.1 & 4.3 & ALL HOSPITALS & 5.0 & 4.8 & 4.8 & 4.5 & 4.2 \\
\hline Ryde & 4.0 & 4.3 & 3.6 & 3.3 & 3.4 & Northern Rivers & & & & & \\
\hline Mater, North Sydney & 5.6 & 5.3 & 5.3 & 5.2 & 5.4 & Grafton Base & 4.4 & 4.8 & 4.5 & 3.9 & 3.9 \\
\hline North Shore Private & - & - & - & 4.8 & 4.8 & Lismore Base & 3.8 & 3.3 & 3.2 & 3.4 & 3.1 \\
\hline Sydney Adventist & 6.2 & 5.9 & 5.6 & 5.3 & 5.5 & Murwillumbah & 4.2 & 4.0 & 4.0 & 3.7 & 3.7 \\
\hline ALL HOSPITALS & 4.9 & 4.8 & 4.5 & 4.5 & 4.6 & Tweed Heads & 3.1 & 3.4 & 3.0 & 3.1 & 3.4 \\
\hline \multicolumn{6}{|l|}{ Western Sydney } & Other Area hospitals & 3.6 & 3.6 & 3.9 & 3.4 & 3.2 \\
\hline Auburn & 3.6 & 3.4 & 3.0 & 2.8 & 2.8 & ALL HOSPITALS & 3.8 & 3.6 & 3.5 & 3.5 & 3.4 \\
\hline Blacktown & 3.3 & 3.3 & 3.1 & 3.1 & 3.0 & Mid North Coast & & & & & \\
\hline Westmead & 3.9 & 3.5 & 3.5 & 3.3 & 3.4 & Coffs Harbour & 4.5 & 4.4 & 3.9 & 4.0 & 3.9 \\
\hline The Hills Private & 5.8 & 5.8 & 5.8 & 5.6 & 5.5 & Kempsey & 4.7 & 4.6 & 4.1 & 3.9 & 3.8 \\
\hline Other Area hospitals & 3.9 & 3.5 & 3.5 & - & - & Port Macquarie Base & 3.9 & 3.9 & 3.7 & 3.8 & 4.1 \\
\hline ALL HOSPITALS & 3.9 & 3.7 & 3.6 & 3.5 & 3.5 & Manning Base & 4.3 & 4.3 & 4.5 & 3.9 & 4.1 \\
\hline \multicolumn{6}{|l|}{ Wentworth } & Other Area hospitals & 4.7 & 4.4 & 4.5 & 4.8 & 4.4 \\
\hline Blue Mountains & 3.5 & 3.6 & 3.6 & 3.7 & 3.5 & ALL HOSPITALS & 4.4 & 4.3 & 4.1 & 4.0 & 4.0 \\
\hline Nepean & 3.5 & 3.4 & 3.5 & 3.2 & 3.3 & New England & & & & & \\
\hline Jamison Private & 5.3 & 5.3 & 5.5 & 5.3 & 5.0 & Armidale & 5.1 & 5.1 & 4.7 & 4.4 & 4.4 \\
\hline Hawkesbury & - & 3.8 & 3.8 & 3.5 & 3.4 & Inverell & 3.7 & 3.6 & 3.8 & 3.4 & 3.4 \\
\hline Other Area hospitals & 3.7 & 3.5 & - & - & - & Moree & 4.1 & 3.8 & 3.6 & 4.0 & 3.7 \\
\hline ALL HOSPITALS & 3.7 & 3.7 & 3.9 & 3.6 & 3.6 & Tamworth Base & 3.7 & 3.5 & 3.6 & 3.6 & 3.8 \\
\hline \multicolumn{6}{|l|}{ South Western Sydney } & Other Area hospitals & 4.6 & 4.6 & 4.2 & 4.1 & 4.1 \\
\hline Fairfield & 3.1 & 3.0 & 2.9 & 2.9 & 2.8 & ALL HOSPITALS & 4.3 & 4.2 & 4.0 & 3.9 & 4.0 \\
\hline Liverpool & 3.1 & 3.1 & 3.1 & 2.9 & 3.0 & Macquarie & & & & & \\
\hline Campbelltown & 3.0 & 2.8 & 2.7 & 2.6 & 2.6 & Dubbo Base & 3.3 & 3.2 & 3.0 & 3.0 & 2.9 \\
\hline Bankstown-Lidcombe & 3.0 & 3.0 & 2.8 & 2.8 & 2.9 & Mudgee & 3.6 & 3.6 & 3.3 & 3.5 & 3.2 \\
\hline Bankstown Private & 5.3 & 5.0 & 5.4 & 4.9 & 4.7 & Other Area hospitals & 3.4 & 3.2 & 3.3 & 3.5 & 3.1 \\
\hline Bowral & 3.7 & 3.2 & 3.0 & 3.0 & 3.0 & ALL HOSPITALS & 3.3 & 3.3 & 3.1 & 3.1 & 2.9 \\
\hline Other Area hospitals & 3.7 & 3.4 & 3.8 & 3.3 & 4.2 & Mid Western & & & & & \\
\hline ALL HOSPITALS & 3.2 & 3.2 & 3.1 & 3.0 & 2.9 & Bathurst Base & 4.2 & 3.7 & 3.2 & 3.3 & 3.4 \\
\hline \multicolumn{6}{|l|}{ Central Coast } & Lithgow & 4.1 & 5.3 & 4.5 & 4.4 & 4.4 \\
\hline Gosford & 3.7 & 3.5 & 3.1 & 2.4 & 2.5 & Orange Base & 3.4 & 3.1 & 3.4 & 3.1 & 3.4 \\
\hline Wyong & - & - & 3.2 & 2.5 & 2.4 & Parkes & 4.5 & 4.2 & 3.9 & 3.9 & 3.7 \\
\hline North Gosford Private & 6.1 & 6.2 & 5.9 & 5.9 & 5.6 & Other Area hospitals & 4.5 & 4.7 & 4.1 & 3.8 & 4.0 \\
\hline ALL HOSPITALS & 4.2 & 4.1 & 3.7 & 3.1 & 3.1 & ALL HOSPITALS & 4.0 & 3.9 & 3.7 & 3.5 & 3.6 \\
\hline \multicolumn{6}{|l|}{ Hunter } & Far West & & & & & \\
\hline Maitland & 3.5 & 3.2 & 3.1 & 3.2 & 3.4 & Broken Hill Base & 4.3 & 4.1 & 3.8 & 4.1 & 4.4 \\
\hline Muswellbrook & 3.9 & 3.9 & 3.8 & 3.5 & 3.5 & Other Area hospitals & 3.2 & 3.8 & 2.9 & 2.8 & 3.6 \\
\hline Belmont & 3.6 & 3.3 & 3.5 & 3.5 & 3.6 & ALL HOSPITALS & 4.0 & 4.1 & 3.6 & 3.8 & 4.2 \\
\hline Singleton & 4.2 & 3.7 & 3.3 & 3.5 & 3.3 & Greater Murray & & & & & \\
\hline John Hunter & 3.9 & 3.7 & 4.0 & 3.9 & 3.6 & Deniliquin & 5.3 & 5.3 & 4.8 & 4.4 & 4.4 \\
\hline Christo Road Private & 5.7 & 5.7 & 5.8 & 5.5 & 5.3 & Griffith Base & 4.1 & 3.6 & 3.4 & 3.4 & 3.1 \\
\hline Other Area hospitals & 4.8 & 4.8 & 4.7 & 4.8 & 4.1 & Wagga Wagga Base & 4.0 & 3.7 & 3.4 & 3.3 & 3.8 \\
\hline ALL HOSPITALS & 4.0 & 3.9 & 4.1 & 4.0 & 3.8 & Calvary, Wagga Wagga & 5.9 & 6.3 & 6.5 & 5.5 & 5.2 \\
\hline \multicolumn{6}{|l|}{ Illawarra } & Other Area hospitals & 4.5 & 4.3 & 4.1 & 3.9 & 3.9 \\
\hline Shoalhaven & 2.9 & 2.7 & 2.3 & 2.5 & 2.7 & ALL HOSPITALS & 4.5 & 4.4 & 4.2 & 3.9 & 3.9 \\
\hline Shellharbour & 3.5 & 3.8 & 3.3 & 3.0 & 2.8 & Southern & & & & & \\
\hline Wollongong & 2.5 & 2.4 & 2.4 & 2.6 & 2.8 & Bega & 4.4 & 4.2 & 4.2 & 4.0 & 3.5 \\
\hline Illawarra Private & 6.3 & 5.6 & 6.3 & 5.6 & 5.6 & Goulburn Base & 4.1 & 3.6 & 3.8 & 3.3 & 3.5 \\
\hline Other Area hospitals & 4.2 & 4.4 & 3.8 & 3.7 & 3.6 & Queanbeyan & 3.4 & 3.2 & 3.2 & 3.4 & 3.4 \\
\hline ALL HOSPITALS & 2.9 & 2.7 & 2.5 & 3.0 & 3.2 & Other Area hospitals & 4.2 & 4.1 & 3.8 & 3.9 & 3.8 \\
\hline & & & & & & ALL HOSPITALS & 4.0 & 3.8 & 3.7 & 3.7 & 3.6 \\
\hline & & & & & & TOTAL NSW & 4.1 & 4.0 & 3.9 & 3.7 & 3.7 \\
\hline
\end{tabular}

Source: NSW Midwives Data Collection (HOIST). Epidemiology and Surveillance Branch, NSW Department of Health.

\# Hospitals with more than 200 deliveries are identified individually. All hospitals include all public and private hospitals. 


\subsection{INDICATORS OF OBSTETRIC CARE}

The Australian Council on Healthcare Standards and the Royal Australian and New Zealand College of Obstetricians and Gynaecologists have endorsed seven clinical indicators for use in Hospitals.
Table 113 shows aggregate information for these indicators for all NSW hospitals and comparative information for all participating hospitals in Australia.

\section{TABLE 113}

CLINICAL INDICATORS FOR OBSTETRICS, NSW AND AUSTRALIA, 2000

Indicator description

\begin{tabular}{cccc} 
NSW & & $\begin{array}{c}\text { Australia } \\
\text { 20th }\end{array}$ & 80th \\
$\%$ & $\%$ & Centile Centile \\
\hline
\end{tabular}

Indicator 1: Induction of labour for other than defined indications.\#

1.1 Mothers undergoing induction of labour for other than defined indications as a percentage of all mothers undergoing induction of labour for any reason.

$\begin{array}{rrrr}31.2 & 33.0 & 20.4 & 49.4 \\ 7.4 & 8.5 & 4.6 & 14.5\end{array}$

1.2 Mothers undergoing induction of labour for other than defined indications as a percentage of all mothers giving birth.

Indicator 2: The rate of vaginal delivery after primary caesarean section.

2.1 Mothers delivering vaginally at the birth immediately following primary caesarean section as a percentage of all mothers delivering at the birth immediately following primary caesarean section.

Indicator 3: Primary caesarean section for failure to progress.

3.1 Mothers undergoing primary caesarean section for failure to progress after a period of labour with cervical dilation of $3 \mathrm{~cm}$ or less as a percentage of all mothers undergoing primary non-elective caesarean section.

3.2 Mothers undergoing primary caesarean section for failure to progress after a period of labour with cervical dilation of more than $3 \mathrm{~cm}$ as a percentage of all mothers undergoing primary non-elective caesarean section.

Indicator 4: Primary caesarean section for fetal distress.

4.1 Mothers undergoing primary caesarean section for fetal distress as a percentage of total mothers delivering.

4.2 Mothers undergoing primary caesarean section for fetal distress as a percentage of

Indicator 5: Incidence of intact lower genital tract in vaginal deliveries.

5.1 Primiparous mothers not requiring surgical repair of the lower genital tract as a percentage of all primiparous mothers.

Indicator 6: Apgar score.

6.1 Infants born with an Apgar score of four or less at five minutes post delivery as a percentage of all infants born."\#

6.2 Infants born with an Apgar score of six or less at ten minutes post delivery as a percentage of all infants born.\#\#

Indicator 7: Term infants transferred or admitted to a neonatal intensive care unit for reasons other than congenital abnormality.\#\#\#

7.1 Term infants admitted to a neonatal intensive care unit for reasons other than congenital abnormality as a percentage of all term infants born.

Source: NSW Midwives Data Collection (HOIST). Epidemiology and Surveillance Branch, NSW Department of Health. The Australian Council on Healthcare Standards. Determining the Potential to Imrpove the Quality of Care in Australian Health Care Organisations. 2nd Edition, Trends in Quality of Care: Results of the ACHS Clinical Indicators 1998-2000 Version 1. Sydney: The Australian Council on Healthcare Standards, 2001.

\# Defined indications include: diabetes, hypertensive disease, fetal distress, fetal death, chorioamnionitis, blood group isoimmunisation, prelabour rupture of membranes, prolonged pregnancy (41 or more weeks), and suspected intrauterine growth retardation.

\#\# NSW denominator includes live births only.

\#\#\# NSW data not collected.

\#\#\#\# NSW data are provided by hospital of birth and may be under-enumerated. Infants transferred to another hospital and then admitted to NICU for reasons other than congenital abnormality may not be reported by the hospital of birth. 


\section{PART 8: REVIEW OF PERINATAL DEATHS 2000}

\subsection{INTRODUCTION}

This chapter presents the results of perinatal death reviews carried out by the NSW Maternal and Perinatal Committee for deaths among babies born in 2000, and the results of a survey of hospital perinatal death review practices, which was carried out during 2001.

Information on the review of perinatal deaths occurring in 2000 is presented in sections 8.2 to 8.7. Information on the the survey of hospital perinatal death review practices is presented in section 8.8

\subsection{PERINATAL DEATH REVIEWS, 2000}

Perinatal deaths in NSW are reviewed by the NSW Maternal and Perinatal Committee, which is a quality assurance committee established under the Health Administration Act 1982, and is privileged under the Act to carry out confidential reviews of both maternal and perinatal deaths.

NSW Department of Health Circular No. 99/101 describes hospital procedures for review and reporting of perinatal deaths. The circular is available on the Department's web site at: www.health.nsw.gov.au/fcsd/rmc/cib/circulars/1999. Under this policy, the NSW Maternal and Perinatal Committee carries out reviews of perinatal deaths occurring among fetuses or infants of at least 22 weeks gestation or at least 500 grams birthweight. The criteria used by the NSW Midwives Data Collection for reporting of births is at least 400 grams birthweight or at least 20 weeks gestation. The Maternal and Perinatal Committee reviews deaths that have a slightly higher threshold to focus attention on deaths which are more likely to be preventable.

Perinatal deaths occurring in 2000 were reviewed by the Committee's Perinatal Outcomes Working Party. Both stillbirths and neonatal deaths were classified according to an obstetric cause-specific classification (amended Whitfield classification). Neonatal deaths were also classified by neonatal cause.

Of the 715 perinatal deaths of at least 22 weeks gestation or at least 500 grams birthweight reported to the NSW Midwives Data Collection, confidential reports on 601 (84.1 per cent) were reviewed and classified. Of the 601 confidential reports received, 421 (70.0 per cent) were for stillbirths and 180 (30.0 per cent) were for neonatal deaths.

\subsection{OBSTETRIC CAUSES OF PERINATAL DEATH}

Overall, almost one third of deaths reviewed (34.6 per cent) were unexplained, and among term infants over half (56.8 per cent) were unexplained (Table 114). Eighteen per cent of deaths followed spontaneous preterm labour, while 13.6 per cent were due to a congenital abnormality of the fetus.

\section{Spontaneous preterm}

There were 106 perinatal deaths associated with spontaneous preterm birth, which comprises normally formed babies born before 37 weeks gestation. Spontaneous preterm birth was attributed to previous spontaneous rupture of membranes in 34 cases, was idiopathic in 26 cases, was associated with a multiple pregnancy in 24 cases and cervical incompetence in 13 cases.

All of these babies were born before 33 weeks gestation and 88 ( 83.0 per cent) were born before 26 weeks gestation.

In 34 cases (32.1 per cent) there was bleeding during pregnancy, of which eight were associated with placental abruption, seven with threatened miscarriage, two with placenta praevia, and the remainder were of unknown origin. In 28 (26.4 per cent) cases there was associated chorioamnionitis.

\section{Intrauterine growth restriction (IUGR)}

In seven cases, the main obstetric cause was found to be IUGR, defined as less than the 10th percentile of birthweight for gestational age with no major congenital abnormalities. Stillbirths with evidence of maceration were not classified as IUGR unless there was evidence of IUGR on serial ultrasound during pregnancy.

Of these seven cases, two were stillbirths. One stillborn baby died during labour and the other had evidence of IUGR on serial ultrasound during pregnancy with placental infarction found at delivery. Among the five neonatal deaths, one was the second of twins in an otherwise uneventful pregnancy, one had evidence of chorioamnionits but no fetal infection, one mother had chronic hypertension, one mother had renal disease, and there were no associated conditions reported in the remaining case.

\section{Unexplained intrauterine death}

The cause of death could not be adequately explained in 208 stillbirths. Of these, 127 (61.1 per cent) were low birthweight and 124 (59.6 per cent) were premature.

There were a variety of maternal conditions reported in this group, including: multiple pregnancy (six cases), maternal hypertension (18), diabetes (nine), history of drug dependency or abuse (four), and cholestasis (one).

Placental histopathology reports were provided in 154 (74.0 per cent) cases, of which 65 (42.2 per cent) were normal. Abnormalities found included: infarction in 24 (15.6 per cent) with 6 showing infarction of 25 per cent or more of the placenta; chorioamnionitis ( $n=13,8.4$ per cent); chorionitis $(n=9,5.8$ per cent); and haemorrhage $(n=5,3.2$ per cent). 


\section{TABLE 114}

\section{PERINATAL DEATHS BY OBSTETRIC CAUSE AND GESTATIONAL AGE, NSW 2000}

Obstetric cause

Gestational age (weeks)

\begin{tabular}{|c|c|c|c|c|c|c|c|}
\hline & \multicolumn{2}{|c|}{ Less than 37} & \multicolumn{2}{|c|}{$37+$} & \multicolumn{2}{|c|}{ TOTAL } \\
\hline & & No. & $\%$ & No. & $\%$ & No. & $\%$ \\
\hline \multirow[t]{8}{*}{1.} & Spontaneous preterm & & & & & & \\
\hline & Multiple pregnancy & 24 & 5.3 & 0 & 0.0 & 24 & 4. \\
\hline & Previous bleeding & 8 & 1.8 & 0 & 0.0 & 8 & 1 \\
\hline & Previous spontaneous rupture of membranes & 34 & 7.5 & 0 & 0.0 & 34 & 5 \\
\hline & Cervical incompetence & 13 & 2.9 & 0 & 0.0 & 13 & 2 \\
\hline & Idiopathic & 26 & 5.7 & 0 & 0.0 & 26 & 4 \\
\hline & Other & 1 & 0.2 & 0 & 0.0 & 1 & 0 \\
\hline & Total & 106 & 23.4 & 0 & 0.0 & 106 & 17.6 \\
\hline 2. & Intrauterine growth retardation (IUGR) & 5 & 1.1 & 2 & 1.4 & 7 & \\
\hline 3. & Unexplained intrauterine death & 124 & 27.4 & 84 & 56.8 & 208 & 34. \\
\hline 4. & Birth trauma & 1 & 0.2 & 0 & 0.0 & 1 & 0 \\
\hline \multirow[t]{7}{*}{5.} & Intrapartum asphyxia & & & & & & \\
\hline & Vaginal delivery & 0 & 0.0 & 3 & 2.0 & 3 & \\
\hline & Cord complications & 2 & 0.4 & 1 & 0.7 & 3 & \\
\hline & Caesarean section & 0 & 0.0 & 3 & 2.0 & 3 & \\
\hline & Forceps delivery & 0 & 0.0 & 2 & 1.4 & 2 & 0 \\
\hline & Ventouse delivery & 0 & 0.0 & 2 & 1.4 & 2 & 0 \\
\hline & Total & 2 & 0.4 & 11 & 7.4 & 13 & \\
\hline
\end{tabular}

\section{Hypertension}

Unspecified

Pre-existing hypertension

Pre-eclamptic toxemia

Pre-existing + PET

Total

$\begin{array}{rl}2 & 0.4 \\ 5 & 1.1 \\ 18 & 4.0 \\ 2 & 0.4 \\ 27 & 6.0\end{array}$

\section{Maternal disease}

Other

Maternal injury

Diabetes/gestational diabetes

Maternal death

Total

\section{Antepartum haemorrhage}

Placental abruption

Vasa praevia

APH undetermined origin

Total

$\%$

\section{Fetal abnormality}

Central nervous system

Cardiovascular system

Urinary tract

Gastrointestinal system

Chromosomal

Metabolic

Multiple

Other

Total

2.7
0.0
3.4
0.0
6.1

4.0

10. Haemolytic disease

Rhesus incompatibility

Total

$\begin{array}{lrl}1.4 & 9 & 1.5 \\ 0.7 & 5 & 0.8 \\ 2.0 & 4 & 0.7 \\ 0.0 & 1 & 0.2 \\ 4.1 & 19 & 3.2\end{array}$

1.5
0.8
0.7
0.2
3.2

11. Infection

Unspecified

Streptococcus Group B

E Coli

Other bacterial

Total

Source: NSW Maternal and Perinatal Committee, NSW Department of Health. 
TABLE 113 (continued)

PERINATAL DEATHS BY OBSTETRIC CAUSE AND GESTATIONAL AGE, NSW 2000

\begin{tabular}{|c|c|c|c|c|c|c|}
\hline \multirow[t]{3}{*}{ Obstetric cause } & \multicolumn{6}{|c|}{ Gestational age (weeks) } \\
\hline & \multicolumn{2}{|c|}{ Less than 37} & \multicolumn{2}{|c|}{$37+$} & \multicolumn{2}{|c|}{ TOTAL } \\
\hline & No. & $\%$ & No. & $\%$ & No. & $\%$ \\
\hline \multicolumn{7}{|l|}{ 12. Other } \\
\hline Non-immune hydrops & 6 & 1.3 & 0 & 0.0 & 6 & 1.0 \\
\hline Feto-maternal haemorrhage & 0 & 0.0 & 1 & 0.7 & 1 & 0.2 \\
\hline Twin to twin transfusion & 12 & 2.6 & 0 & 0.0 & 12 & 2.0 \\
\hline Maternal drug dependence/abuse & 2 & 0.4 & 0 & 0.0 & 2 & 0.3 \\
\hline Unknown/unexplained & 1 & 0.2 & 0 & 0.0 & 1 & 0.2 \\
\hline Other & 4 & 0.9 & 2 & 1.4 & 6 & 1.0 \\
\hline Total & 25 & 5.5 & 3 & 2.0 & 28 & 4.7 \\
\hline TOTAL & 453 & 100.0 & 148 & 100.0 & 601 & 100.0 \\
\hline
\end{tabular}

Source: NSW Maternal and Perinatal Committee, NSW Department of Health.

\section{Birth trauma}

This group comprises normally formed babies of at least 1,500 grams birthweight with evidence of lethal trauma at autopsy. There was only one death attributed to birth trauma. This 36 weeks gestation baby had a forceps delivery and died in the neonatal period following a subdural haemorrhage.

\section{Intrapartum asphyxia}

There were 13 deaths of normally formed babies of at least 1,500 grams birthweight with evidence of intrapartum hypoxia. Eight babies were stillborn following death during labour and five deaths occurred in the neonatal period.

There were two cases of shoulder dystocia, two cases of cord prolapse one of which was a first twin, and two cases of meconium aspiration. One case of a true knot in the cord resulted in death during labour. Another intrapartum death followed uterine rupture during labour. One baby, a second twin, died during labour following a pregnancy complicated by pre-eclampsia and placental abruption. There was one death associated with chorioamnionitis and one with maternal diabetes. Two intrapartum deaths were unexplained.

\section{Hypertension}

Thirty-six deaths were associated with hypertension: preeclampsia (23), chronic hypertension (five), combined chronic hypertension and pre-eclampsia (two) and unspecified hypertension (six).

Twenty-two deaths were among stillbirths and 11 were neonatal deaths. Three deaths were among babies of twin pregnancies.

Six deaths in this group were associated with placental abruption. One case of pre-eclampsia was complicated by eclampsia and placental abruption during labour. One mother had antiphospholipid antibodies and chronic hypertension complicated by pre-eclamspia. Another had both hypertension and diabetes.
Placental histopathology was reported for 28 (77.8 per cent), of which eight were normal. Infarction was reported in 14 cases, with four of these showing infarction of 25 per cent or more of the placenta. Other abnormalities reported were: haemorrhage (four), chorioamnionitis (one), and ischaemic changes (one).

\section{Maternal disease}

Nineteen deaths were attributed to other maternal diseases including: maternal injury following motor vehicle accident (four); diabetes (four); presence of antiphospholipid antibodies (six), in one case associated with systemic lupus erythematosis and diabetes; renal failure (one); presence of anti-cardiolipin antibodies (one); the presence of a large uterine fibroid which had undergone hyaline necrosis (one), maternal assault (one), and maternal death from a cause unrelated to pregnancy (one).

\section{Antepartum haemorrhage}

Forty-six deaths were due to antepartum haemorrhage, of which 37 were due to placental abruption, one due to vasa praevia and eight were of undetermined origin.

Thirty-three deaths were stillborn babies who died before the onset of labour, and 13 were neonatal deaths.

Two antepartum haemorrhages were associated with maternal diabetes, one mother was positive for antinuclear antibody (1:60) and one had an abnormal protein c profile.

\section{Fetal abnormality}

Eighty-two deaths were found to be due to fetal abnormalities. Chromosal defects were most common $(n=29,35.8$ per cent), of which 11 were trisomy 21 , nine were trisomy 18 , four were trisomy 13 , and five were other chromosommal abnormalities. The next most common group of abnormalities were of the central nervous system ( $n=15,18.5$ per cent), of which seven were neural tube defects, and four were congenital hydrocephalus. Nine babies had multiple abnormalities not associated with a chromosomal abnormality. 


\section{Haematological disease}

One death due to rhesus incompatibility was reported.

\section{Infection}

Fifty-four deaths were found to be due to infection, of which 30 were stillbirths and 24 were neonatal deaths. In all 30 stillbirths and in 17 neonatal deaths an associated chorioamnionitis was reported.

Most commonly an organism was not specified $(n=36$, 66.7 per cent). Organisms specified included Streptococcus group B (12), E. Coli (four), multi-resistant Staphylococcus Aureus (one), and an unspecified spirochaete (one).

\section{Other}

Twenty-eight deaths were due to other causes including: twin-to-twin transfusion (12); non-immune fetal hydrops (six); sacrococcygeal teratoma (two); maternal drug dependence/abuse (two); feto-maternal haemorrhage (one); accident in the post-natal period (one); termination of pregnancy for oligohydramnios (one); ovarian pregnancy (one), and fetal demise in utero due to true knot in cord (one). The placenta of one case, which was classified as unexplained, showed haemorrhagic endovasculitis on histopathology.

\subsection{OBSTETRIC CAUSE OF PERINATAL DEATH BY HOSPITAL SIZE}

The majority of perinatal deaths occurred in hospitals with more than 2000 births in 2000 (Table 115). The proportion of unexplained intrauterine deaths was substantially higher in hospitals with less than 1500 births per year compared with larger hospitals, possibly due to difficulties with access to perinatal postmortem services. Conversely, the proportion of deaths associated with congenital abnormalities was highest in hospitals with greater than 2000 births per year, reflecting patterns of referral for diagnosis and treatment.

\subsection{NEONATAL CAUSE OF DEATH}

Of the 180 neonatal deaths, 157 (87.2 per cent) were less than 37 weeks gestation (Table 116). The most common neonatal cause of death, affecting 43.9 per cent of infants, was extreme prematurity. All these infants were less than 26 weeks gestation. Thirty-two infants died from a congenital abnormality. The other most common causes of death included infection (11 deaths), neurological complications including asphyxia (11) and haemorrhage (nine), and hyaline membrane disease (nine).

\subsection{PERINATAL DEATHS ASSOCIATED WITH MATERNAL DRUG DEPENDENCY/ABUSE}

Two perinatal deaths were attributed to maternal drug dependency or drug abuse. One was attributed to the use of 'speed' and the other of heroin.

A further 13 perinatal deaths occurred among babies of mothers reported to have a history of drug dependency or abuse, but drug use was not considered to be the main cause of death.

\subsection{POSTMORTEM EXAMINATION}

Postmortem examination is valuable in ascertaining or confirming the cause of death, and identifying additional factors which may have contributed to the death.

In 2000, postmortem examinations were carried out in 196 (32.6 per cent) cases of perinatal death. There were 156 postmortems carried out among stillborn infants (37.1 per cent of all stillbirths), and 40 postmortems among babies who died in the neonatal period (20.4 per cent of all neonatal deaths). Placental histopathology was carried out in 429 (71.4 per cent) perinatal deaths .

\section{TABLE 115}

PERINATAL DEATHS BY OBSTETRIC CAUSE AND HOSPITAL SIZE, NSW 2000

\begin{tabular}{|c|c|c|c|c|c|c|c|c|c|c|c|c|}
\hline \multirow[t]{3}{*}{ Obstetric cause } & \multicolumn{12}{|c|}{ Hospital size (No. births per year) } \\
\hline & \multicolumn{2}{|c|}{$0-499$} & \multicolumn{2}{|c|}{ 500-999 } & \multicolumn{2}{|c|}{ 1000-1499 } & \multicolumn{2}{|c|}{ 1500-1999 } & \multicolumn{2}{|c|}{$2000+$} & \multicolumn{2}{|c|}{ TOTAL } \\
\hline & No. & $\%$ & No. & $\%$ & No. & $\%$ & No. & $\%$ & No. & $\%$ & No. & $\%$ \\
\hline 1. Spontaneous preterm & 9 & 18.4 & 14 & 16.5 & 5 & 8.9 & 16 & 31.4 & 62 & 17.2 & 106 & 17.6 \\
\hline 2. Intrauterine growth retardation (IUGR) & 1 & 2.0 & 1 & 1.2 & 0 & 0.0 & 1 & 2.0 & 4 & 1.1 & 7 & 1.2 \\
\hline 3. Unexplained intrauterine death & 21 & 42.9 & 38 & 44.7 & 26 & 46.4 & 14 & 27.5 & 109 & 30.3 & 208 & 34.6 \\
\hline 4. Birth trauma & 0 & 0.0 & 0 & 0.0 & 0 & 0.0 & 0 & 0.0 & 1 & 0.3 & 1 & 0.2 \\
\hline 5. Intrapartum asphyxia & 3 & 6.1 & 2 & 2.4 & 1 & 1.8 & 1 & 2.0 & 6 & 1.7 & 13 & 2.2 \\
\hline 6. Hypertension & 3 & 6.1 & 6 & 7.1 & 1 & 1.8 & 6 & 11.8 & 20 & 5.6 & 36 & 6.0 \\
\hline 7. Maternal disease & 1 & 2.0 & 1 & 1.2 & 2 & 3.6 & 1 & 2.0 & 14 & 3.9 & 19 & 3.2 \\
\hline 8. Antepartum haemorrhage & 6 & 12.2 & 8 & 9.4 & 5 & 8.9 & 0 & 0.0 & 27 & 7.5 & 46 & 7.7 \\
\hline 9. Fetal abnormality & 1 & 2.0 & 9 & 10.6 & 7 & 12.5 & 4 & 7.8 & 61 & 16.9 & 82 & 13.6 \\
\hline 10. Haemolytic disease & 0 & 0.0 & 0 & 0.0 & 0 & 0.0 & 0 & 0.0 & 1 & 0.3 & 1 & 0.2 \\
\hline 11. Infection & 3 & 6.1 & 4 & 4.7 & 6 & 10.7 & 7 & 13.7 & 34 & 9.4 & 54 & 9.0 \\
\hline 12. Other & 1 & 2.0 & 2 & 2.4 & 3 & 5.4 & 1 & 2.0 & 21 & 5.8 & 28 & 4.7 \\
\hline TOTAL & 49 & 100.0 & 85 & 100.0 & 56 & 100.0 & 51 & 100.0 & 360 & 100.0 & 601 & 100.0 \\
\hline
\end{tabular}

Source: NSW Maternal and Perinatal Committee, NSW Department of Health. 


\section{TABLE 116}

NEONATAL DEATHS BY CAUSE AND GESTATIONAL AGE, NSW 2000

\begin{tabular}{|c|c|c|c|c|c|c|}
\hline \multirow{3}{*}{ Neonatal cause } & \multicolumn{6}{|c|}{ Gestational age (weeks) } \\
\hline & \multicolumn{2}{|c|}{ Less than 37} & \multicolumn{2}{|c|}{$37+$} & \multicolumn{2}{|c|}{ TOTAL } \\
\hline & No. & $\%$ & No. & $\%$ & No. & $\%$ \\
\hline \multicolumn{7}{|l|}{ Extreme prematurity } \\
\hline Life support initiated & 19 & 12.1 & 0 & 0.0 & 19 & 10.6 \\
\hline Life support not initiated & 35 & 22.3 & 0 & 0.0 & 35 & 19.4 \\
\hline Life support not stated & 15 & 9.6 & 0 & 0.0 & 15 & 8.3 \\
\hline Total & 69 & 43.9 & 0 & 0.0 & 69 & 43.9 \\
\hline Congenital abnormality & 22 & 14.0 & 10 & 43.5 & 32 & 17.8 \\
\hline \multicolumn{7}{|l|}{ Neurological } \\
\hline Asphyxia & 5 & 3.2 & 6 & 26.1 & 11 & 6.1 \\
\hline Haemorrhage & 9 & 5.7 & 0 & 0.0 & 9 & 5.0 \\
\hline Other & 1 & 0.6 & 0 & 0.0 & 1 & 0.6 \\
\hline Total & 15 & 9.6 & 6 & 26.1 & 21 & 11.7 \\
\hline \multicolumn{7}{|l|}{ Cardio-respiratory } \\
\hline Hyaline membrane disease & 9 & 5.7 & 0 & 0.0 & 9 & 5.0 \\
\hline Persistent pulmonary hypertension & 2 & 1.3 & 0 & 0.0 & 2 & 1.1 \\
\hline Necrotising enterocolitis & 1 & 0.6 & 0 & 0.0 & 1 & 0.6 \\
\hline Other & 9 & 5.7 & 2 & 8.7 & 11 & 6.1 \\
\hline Total & 21 & 13.4 & 2 & 8.7 & 23 & 12.8 \\
\hline Metabolic/ endocrine & 0 & 0.0 & 1 & 4.3 & 1 & 0.6 \\
\hline \multicolumn{7}{|l|}{ Infection } \\
\hline Congenital bacterial & 6 & 3.8 & 1 & 4.3 & 7 & 3.9 \\
\hline Acquired bacterial & 3 & 1.9 & 0 & 0.0 & 3 & 1.7 \\
\hline Unspecified & 1 & 0.6 & 0 & 0.0 & 1 & 0.6 \\
\hline Total & 10 & 6.4 & 1 & 4.3 & 11 & 6.1 \\
\hline Other & 15 & 9.6 & 1 & 4.3 & 16 & 8.9 \\
\hline Not stated & 5 & 3.2 & 2 & 8.7 & 7 & 3.9 \\
\hline TOTAL & 157 & 100.0 & 23 & 100.0 & 180 & 100.0 \\
\hline
\end{tabular}

Source: NSW Maternal and Perinatal Committee, NSW Department of Health. 


\subsection{SURVEY OF PERINATAL DEATH REVIEW PROCEDURES IN NSW HOSPITALS}

\section{Introduction}

A Department of Health policy on Hospital Procedures for Review and Reporting of Perinatal Deaths (Circular 99/101) was issued in December 1999. The policy provides for the establishment of Perinatal Death Review Committees at hospital or health area level as a quality assurance activity.

A survey was carried out to determine the extent to which perinatal death review procedures were in place and to identify any problems.

\section{Method}

In early 2001, a questionnaire concerning perinatal death review procedures was sent to all hospitals that reported births to the MDC in 1999. The questionnaire asked about the existence of a perinatal death review committee at the hospital or elswhere, it's composition, meeting frequency, privilege under the NSW Health Administration Act 1982, and any problems.

Returned questionnaires were entered into a MS Access database and analysed using SAS version 8.1. Information on types of perinatal death review practices were grouped and compared with the numbers of births and perinatal deaths reported to the MDC for 2000. Information on births and perinatal deaths for hospitals that opened or closed in 2000 were excluded from the analysis.

\section{Results}

Of the 143 hospitals in NSW that were open for the full year and reported at least one birth in 2000, 115 responded to the survey, giving a response rate of 80.4 per cent.

A variety of processes were reported for perinatal death reviews (Table 117). Reviews were carried out by a perinatal death review committee in 53 hospitals, as part of a wider clinical review meeting in 15 hospitals, on an informal basis in six hospitals, by a quality assurance commitee in one hospital, and a medical review panel in one hospital. Twenty-seven hospitals reported not having any review process in place, of which 25 (92.6 per cent) were rural hospitals and 22 (81.5 per cent) had less than 200 births per year. Eleven hospitals reported that they either do not or no longer offer an obstetric service.

The type of perinatal death review process was compared with the numbers of births and perinatal deaths reported from hospitals in 2000 (Table 118). Seventy-five per cent of births and 83.3 per cent of perinatal deaths occurred in hospitals with a designated perinatal death review committee, and 90.5 per cent of births and 92.7 per cent of perinatal deaths occurred in hospitals with some sort of perinatal death process in place.

Of the 76 hospitals that carried out perinatal death reviews, 66 (86.8 per cent) carried out their own reviews, six contributed to reviews carried out at another hospital, for three hospitals reviews were carried out at area health service level, and one hospital reported having a joint committee with another hospital.

In the 70 hospitals with committee-based reviews, 15 met at least monthly, 33 quarterly, three met 5-6 times per year, nine met twice a year and eight met as necessary. The meeting frequency for two hospitals was not stated.

Eight hospitals had applied for and gained privilege under the Health Administration Act 1982 for their committee's reviews of confidential medical information. A further three hospitals had submitted an application for privilege.

Hospitals with perinatal death review procedures in place did not report any major problems. A small number of hospitals commented on difficulties, including:

- difficulties in getting busy clinicians together for meetings;

- concern about deliberations of committee being subpoenaed, and need for committee's to have privilege to carry out reviews;

- difficulties in obtaining information from referral hospitals where death occurs following transfer;

- completing the paperwork associated with submitting the Confidential Report Forms on Perinatal Death to the Department of Health;

- obtaining files from medical records departments for purpose not directly related to clinical care.

Of the 28 hospitals that did not have perinatal death review procedures in place, 13 considered that a local perinatal death committee was not justified due to the low number of births and/or perinatal deaths at the hospital. Three hospitals reported that review procedures were being developed. A further two hospitals were waiting for a decision on whether they would be part of a health area-based review process.

\section{Discussion}

Review of perinatal deaths is an important part of quality assurance in obstetric and neonatal care. This survey found that processes exist in NSW hospitals for the review of over 90 per cent of perinatal deaths in the State. In over 80 per cent of perinatal deaths, reviews would be carried out by a designated perinatal death review committee.

For those hospitals with established review processes, there were no major problems reported. Some hospitals had difficulties with administrative arrangements associated with convening the meeting, obtaining relevant papers and providing Confidential Reports to the Department of Health for review by the NSW Maternal and Perinatal Committee. 


\section{TABLE 117}

TYPE OF PERINATAL DEATH REVIEW BY HOSPITAL SIZE, NSW 2000

\begin{tabular}{|c|c|c|c|c|c|c|c|c|c|c|c|c|}
\hline \multirow[t]{3}{*}{ Hospital perinatal death reviews } & \multicolumn{12}{|c|}{ Hospital size (No. births per year) } \\
\hline & \multicolumn{2}{|c|}{$0-499$} & \multicolumn{2}{|c|}{ 500-999 } & \multicolumn{2}{|c|}{$1000-1499$} & \multicolumn{2}{|c|}{$1500-1999$} & \multicolumn{2}{|c|}{$2000+$} & \multicolumn{2}{|c|}{ TOTAL } \\
\hline & No. & $\%$ & No. & $\%$ & No. & $\%$ & No. & $\%$ & No. & $\%$ & No. & $\%$ \\
\hline Perinatal death review committee & 18 & 18.8 & 14 & 70.0 & 4 & 50.0 & 5 & 83.3 & 12 & 92.3 & 53 & 37.1 \\
\hline Clinical review committee/meeting & 8 & 8.3 & 1 & 5.0 & 4 & 50.0 & 1 & 16.7 & 1 & 7.7 & 15 & 10.5 \\
\hline Informal review & 6 & 6.3 & 0 & 0.0 & 0 & 0.0 & 0 & 0.0 & 0 & 0.0 & 6 & 4.2 \\
\hline Quality assurance committee & 1 & 1.0 & 0 & 0.0 & 0 & 0.0 & 0 & 0.0 & 0 & 0.0 & 1 & 0.7 \\
\hline Medical review panel & 1 & 1.0 & 0 & 0.0 & 0 & 0.0 & 0 & 0.0 & 0 & 0.0 & 1 & 0.7 \\
\hline No review process & 26 & 27.1 & 1 & 5.0 & 0 & 0.0 & 0 & 0.0 & 0 & 00 & 27 & 18.9 \\
\hline No obstetric service & 11 & 11.5 & 0 & 0.0 & 0 & 0.0 & 0 & 0.0 & 0 & 0.0 & 11 & 7.7 \\
\hline Not stated & 25 & 26.0 & 4 & 20.0 & 0 & 0.0 & 0 & 0.0 & 0 & 0.0 & 29 & 20.3 \\
\hline TOTAL & 96 & 100.0 & 20 & 100.0 & 8 & 100.0 & 6 & 100.0 & 13 & 100.0 & 143 & 100.0 \\
\hline
\end{tabular}

Source: Survey of perinatal death review practices in NSW hospitals, 2001.

\# $\quad$ Excludes hospitals that opened or closed in 2000.

\section{TABLE 118}

COVERAGE OF BIRTHS AND PERINATAL DEATHS BY TYPE OF PERINATAL DEATH REVIEW, NSW 2000*

\begin{tabular}{|c|c|c|c|c|}
\hline \multirow[t]{2}{*}{ Type of perinatal death review } & \multicolumn{4}{|c|}{ Coverage } \\
\hline & No. & $\%$ & No. & $\%$ \\
\hline Perinatal death review committee & 64797 & 74.7 & 709 & 83.3 \\
\hline Clinical review committee/meeting & 12395 & 14.3 & 73 & 8.6 \\
\hline Informal review & 563 & 0.6 & 2 & 0.2 \\
\hline Quality assurance committee & 474 & 0.5 & 4 & 0.5 \\
\hline Medical review panel & 319 & 0.4 & 1 & 0.1 \\
\hline No review process & 3634 & 4.2 & 24 & 2.8 \\
\hline No obstetric service & 57 & 0.1 & 1 & 0.1 \\
\hline Not stated & 4541 & 5.2 & 37 & 4.3 \\
\hline TOTAL & 86780 & 100.0 & 851 & 100.0 \\
\hline
\end{tabular}

Source: Survey of perinatal death review practices in NSW hospitals, 2001.

\# Excludes hospitals that opened or closed in 2000.

Concerns about confidential information being subpoenaed was reported by one hospital. Hospitals may address this by seeking privilege under the Health Administration Act 1982. The process for seeking privilege is described in Department of Health Circular 99/101.

Twenty five rural hospitals reported that they offer an obstetric service but currently have no perinatal death review processes in place. Most of these hospitals offer low risk birthing services and have either none or very small numbers of perinatal deaths per year. For these hospitals, an association with a referral hospital or health area-based review process would be necessary to support a meaningful perinatal death review process. 


\section{PART 9: RISK-ADJUSTED CAESAREAN SECTION RATES IN NSW HOSPITALS, 2000}

\subsection{INTRODUCTION}

This chapter presents the results of a study that produced risk-adjusted rates of caesarean section for NSW hospitals, using information on clinical risk factors currently collected through the NSW Midwives Data Collection (MDC). The objectives of this study were to:

- describe the relationship between caesarean section and the known risk factors using the MDC;

- validate the logistic regression model developed for prediction;

- facilitate comparison of caesarean section rates by standardisation based on logistic regression models;

- provide information to hospitals about their crude and adjusted caesarean section rates in 2000 .

\subsection{METHODS}

Data were obtained from the MDC for the year 2000. The data set was divided into two parts based on baby's date of birth. Logistic regression models were developed on records of babies born between 1 January and 30 June. The model was then validated using the data on births occurring in the second half of the year.

Predicted probabilities from the final logistic model were applied to individual records for hospitals with 100 or more births during July-December 2000, resulting in each confinement being allocated a probability of caesarean section. The probabilities were summed for each hospital to give the expected number of caesarean sections by hospital, adjusted for the risk factors in the model. Standardised caesarean ratios were calculated for hospitals by calculating the ratio of observed to expected number of caesarean sections. The risk-adjusted caesarean section rate for each hospital was calculated by multiplying its standardised caesarean ratio with the caesarean section rate in the standard population of births (20.8 per cent for the first six months of 2000).

A detailed description of the method used to develop and validate the model is included in the Explanatory Notes of this report (page 16).

\subsection{RESULTS}

The crude caesarean section rate was 20.8 per cent in the first six months of 2000 and 21.8 per cent in the second six months. The mean age of mothers was 29 years in both the first and second half of 2000.

The risk of caesarean section for mothers with gestational diabetes was not significantly different to mothers without gestational diabetes. Table 118 presents crude and adjusted odds ratios for each statistically significant variable and shows:

- the risk of caesarean section rose steadily with increasing age, with the odds of caesarean section increasing by six per cent for each year of maternal age;

- the presence of breech and other malpresentation greatly increased the risk of caesarean section. The risks increased substantially when adjusted for other factors;

- primiparous mothers have higher risk of caesarean section than multiparous mothers;

- multiple births have higher risk of caesarean section;

- the presence of diabetes mellitus, essential hypertension and pregnancy induced hypertension increased the risk of caesarean section;

- mothers who had a previous caesarean section faced a higher risk of caesarean section in the current pregnancy;

- when unadjusted for other factors, gestational age groups 20-28 weeks and 29-39 weeks had increased risk of caesarean section. The risk decreased for 2028 weeks gestation after adjusting for other factors.

Risk-adjusted caesarean section rates varied less than crude rates. Crude rates ranged from 4.4 to 35.6 per cent and adjusted rates ranging from 11.9 to 31.2 per cent across hospitals (Table 119).

The highest crude caesarean section rates were among some tertiary referral hospitals and some private hospitals. After adjusting for clinical risk factors, estimated caesarean section rates in tertiary referral hospitals were no more than three per cent higher than the NSW rate of 21.7 per cent, and in four cases were lower than the NSW rate. The findings were similar for many private hospitals, with adjusted caesarean section rates generally lower than crude rates, and in some cases dramatically lower. Conversely, risk-adjusted caesarean section rates for smaller non-tertiary public hospitals tended to be higher than their crude rates.

\subsection{DISCUSSION}

This study examined clinical risk factors as predictors of caesarean section. We found that a combination of well-known clinical risk factors can predict caesarean section with a high level of certainty: These factors include: maternal age, type of presentation, parity, plurality, maternal diabetes mellitus, essential (chronic) hypertension, pregnancy-induced hypertension, previous caesarean section and gestational age. Of these clinical risk factors, by far the highest odds ratios were found to be associated with malpresentation (adjusted OR: 29.0 for breech and 48.2 for other malpresentation) and previous caesarean section (adjusted $O R=45.1$ ). A moderately high Odds Ratio was also found for primiparous mothers (adjusted $\mathrm{OR}=4.4$ ). 


\section{TABLE 119}

CRUDE AND ADJUSTED ODDS RATIOS FOR CAESAREAN SECTION BY CLINICAL RISK FACTORS FOUND TO BE SIGNIFICANT ON LOGISTIC MODELLING\#

\begin{tabular}{|c|c|c|c|c|c|}
\hline \multirow{2}{*}{$\begin{array}{l}\text { Risk factor } \\
\text { Age (continuous) }\end{array}$} & \multirow{2}{*}{$\begin{array}{c}\begin{array}{c}\text { Births } \\
\%\end{array} \\
\text { n.a }\end{array}$} & \multicolumn{2}{|c|}{$\begin{array}{r}\text { Crude Odds Ratio } \\
95 \% \mathrm{Cl}\end{array}$} & \multicolumn{2}{|c|}{$\begin{array}{c}\text { Adjusted Odds Ratio } \\
95 \% \mathrm{Cl}\end{array}$} \\
\hline & & 1.07 & $1.06-1.07$ & 1.07 & $1.07-1.08$ \\
\hline \multicolumn{6}{|l|}{ Presentation } \\
\hline Breech & 4.2 & 19.43 & $17.24-21.91$ & 29.04 & $25.66-32.86$ \\
\hline Other malpresentation & 0.8 & 23.61 & $17.88-31.18$ & 48.16 & $36.66-63.26$ \\
\hline Vertex & 95.0 & 1.00 & & 1.00 & \\
\hline Primiparous mothers & 41.5 & 1.20 & $1.14-1.25$ & 4.44 & $4.17-4.73$ \\
\hline Multiparous mothers & 58.5 & 1.00 & & 1.00 & \\
\hline Multiple births & 1.7 & 3.53 & $3.05-4.10$ & 2.25 & $1.88-2.68$ \\
\hline Singleton births & 98.3 & 1.00 & & 1.00 & \\
\hline \multicolumn{6}{|l|}{ Diabetes mellitus } \\
\hline Yes & 0.5 & 3.01 & $2.30-3.94$ & 2.11 & $1.54-2.90$ \\
\hline No & 99.5 & 1.00 & & 1.00 & \\
\hline \multicolumn{6}{|l|}{ Essential hypertension } \\
\hline Yes & 1.0 & 2.24 & $1.85-2.72$ & 1.83 & $1.46-2.28$ \\
\hline No & 99.0 & 1.00 & & 1.00 & \\
\hline \multicolumn{6}{|c|}{ Pregnancy induced hypertension } \\
\hline Yes & 7.1 & 1.79 & $1.65-1.94$ & 1.78 & $1.63-1.94$ \\
\hline No & 92.9 & 1.00 & & 1.00 & \\
\hline \multicolumn{6}{|c|}{ Previous caesarean section } \\
\hline Yes & 9.6 & 19.01 & $17.60-20.54$ & 45.14 & $41.46-49.14$ \\
\hline No & 90.4 & 1.00 & & 1.00 & \\
\hline \multicolumn{6}{|l|}{ Gestational age } \\
\hline $20-28$ weeks & 0.7 & 2.21 & $1.71-2.84$ & 0.32 & $0.24-0.44$ \\
\hline 29-39 weeks & 47.6 & 2.29 & $2.18-2.40$ & 1.52 & $1.44-1.60$ \\
\hline $40+$ weeks & 51.7 & 1.00 & & 1.00 & \\
\hline
\end{tabular}

Source: NSW Midwives Data Collection, Epidemiology and Surveillance Branch, NSW Department of Health.

\# Interaction terms not shown.

In the second half of 2000, crude caesarean section rates in NSW hospitals varied from 4.4 to 35.6 per cent. After clinical risk-factor adjustment, the rates varied from 11.9 to 31.2 per cent. As expected, risk-adjustment tended to result in a lower estimate of the caesarean section rate for tertiary referral centres, where the majority of mothers with very high-risk pregnancies give birth. The caesarean section rates for some private hospitals also fell after risk-adjustment and in some cases quite dramatically.

The clinical risk adjustment model developed for this study did not include other factors that may influence a decision for caesarean section, such as private-public insurance status or induction of labour. This was deliberate, as it was intended to examine how well caesarean section could be predicted based on the presence of risk factors whose existence are beyond the control of mother or clinician.

While adjustment for clinical risk factors accounts for much of the variation in caesarean section rates in NSW hospitals, variation persisted after adjustment for a wide range of clinical risk factors. Other factors that could account for the residual variation include: other medical conditions and obstetric complications for which information is not collected by the MDC; quality of care issues in relation to the management of medical conditions and obstetric complications; induction of labour and public-private insurance status, as mentioned above; and maternal or clinician preference.

It is inappropriate to use these risk-adjusted rates to make inferences about quality of care. The risk-adjustment process used in this study is based on the associations between risk factors and caesarean section on average in NSW. Average care in NSW may not, and probably does not, represent clinical best practice. Bearing this in mind, risk-adjusted caesarean section rates do provide more reliable information for comparing hospitals than crude rates, and the modelling of clinical risk factors carried out in this study provides useful information on the strength of various clinical factors to predict caesarean section.

\section{Acknowledgments}

We wish to acknowledge Mr Gaston Arnolda and Professor Geoffrey Berry, whose work on a similar project for the NSW Department of Health in 1994 provided much of the theoretical basis for this study. We also would like to thank Dr Tim Churches for methodogical advice. 


\section{TABLE 120}

CRUDE AND ADJUSTED CAESAREAN SECTION RATES BY HOSPITAL, NSW JULY-DECEMBER 2000\#

\begin{tabular}{|c|c|c|c|c|c|}
\hline $\begin{array}{l}\text { Health Area and } \\
\text { hospital }\end{array}$ & $\begin{array}{c}\text { Caesarean } \\
\text { sections } \\
\text { No. }\end{array}$ & $\begin{array}{c}\text { Births } \\
\text { No. }\end{array}$ & $\begin{array}{c}\text { Crude } \\
\text { rate } \\
\%\end{array}$ & $\begin{array}{c}\text { Adjusted } \\
\text { rate } \\
\%\end{array}$ & $\begin{array}{l}99 \% \text { confidence interval } \\
\text { of adjusted rate }\end{array}$ \\
\hline \multicolumn{6}{|l|}{ Central Sydney } \\
\hline Canterbury & 147 & 775 & 19.0 & 21.3 & $17.4-25.7$ \\
\hline Royal Prince Alfred & 435 & 1881 & 23.1 & 19.2 & $17.1-21.3$ \\
\hline Sydney Private & 28 & 117 & 23.9 & 21.6 & $13.2-32.0$ \\
\hline \multicolumn{6}{|l|}{ Northern Sydney } \\
\hline Hornsby & 114 & 597 & 19.1 & 20.4 & $16.1-25.1$ \\
\hline Manly & 92 & 429 & 21.4 & 19.7 & $15.3-24.8$ \\
\hline Mona Vale & 68 & 312 & 21.8 & 21.5 & $15.9-28.1$ \\
\hline Royal North Shore & 246 & 843 & 29.2 & 22.0 & $19.0-25.2$ \\
\hline Ryde & 77 & 358 & 21.5 & 22.3 & $16.8-28.6$ \\
\hline Mater, North Sydney & 328 & 1031 & 31.8 & 24.0 & $21.2-26.9$ \\
\hline North Shore Private & 329 & 925 & 35.6 & 25.5 & $22.6-28.5$ \\
\hline Sydney Adventist & 288 & 1126 & 25.6 & 21.5 & $18.8-24.5$ \\
\hline \multicolumn{6}{|l|}{ Western Sydney } \\
\hline Auburn & 93 & 668 & 13.9 & 18.4 & $14.1-23.4$ \\
\hline Blacktown & 223 & 1358 & 16.4 & 18.5 & $15.7-21.6$ \\
\hline Westmead & 415 & 1967 & 21.1 & 19.8 & $17.7-22.2$ \\
\hline The Hills Private & 166 & 700 & 23.7 & 21.0 & $17.4-24.8$ \\
\hline Westmead Private & 28 & 123 & 22.8 & 18.0 & $11.0-26.8$ \\
\hline \multicolumn{6}{|l|}{ Wentworth } \\
\hline Blue Mountains & 42 & 203 & 20.7 & 25.7 & $17.2-35.9$ \\
\hline Nepean & 393 & 1760 & 22.3 & 24.4 & $21.6-27.3$ \\
\hline Hawkesbury & 97 & 510 & 19.0 & 21.0 & $16.3-26.3$ \\
\hline Nepean Private & 57 & 228 & 25.0 & 22.4 & $16.1-29.6$ \\
\hline \multicolumn{6}{|l|}{ South Western Sydney } \\
\hline Fairfield & 131 & 1087 & 12.1 & 14.3 & $11.4-17.6$ \\
\hline Liverpool & 243 & 1557 & 15.6 & 18.7 & $15.9-21.7$ \\
\hline Campbelltown & 202 & 1409 & 14.3 & 17.8 & $15.0-21.0$ \\
\hline Bankstown-Lidcombe & 163 & 969 & 16.8 & 19.2 & $15.8-23.0$ \\
\hline Bankstown Private & 27 & 113 & 23.9 & 27.5 & $16.6-41.0$ \\
\hline Sydney Southwest Private & 59 & 285 & 20.7 & 20.3 & $14.6-27.0$ \\
\hline Bowral & 44 & 325 & 13.5 & 15.6 & $10.5-22.1$ \\
\hline \multicolumn{6}{|l|}{ Central Coast } \\
\hline Gosford District & 270 & 1196 & 22.6 & 23.0 & $19.9-26.3$ \\
\hline Wyong & 9 & 206 & 4.4 & 11.9 & $4.2-25.8$ \\
\hline North Gosford Private & 106 & 353 & 30.0 & 24.5 & $19.5-30.0$ \\
\hline \multicolumn{6}{|l|}{ Hunter } \\
\hline Maitland & 157 & 652 & 24.1 & 25.9 & $21.4-30.8$ \\
\hline Muswellbrook District & 12 & 103 & 11.7 & 15.5 & $6.6-29.4$ \\
\hline Belmont & 48 & 292 & 16.4 & 19.8 & $13.5-27.3$ \\
\hline John Hunter & 363 & 1770 & 20.5 & 21.5 & $19.0-24.2$ \\
\hline Christo Road Private & 126 & 476 & 26.5 & 23.1 & $18.7-28.0$ \\
\hline \multicolumn{6}{|l|}{ Illawarra } \\
\hline Shoalhaven & 97 & 412 & 23.5 & 24.9 & $19.4-31.0$ \\
\hline Wollongong & 227 & 1264 & 18.0 & 21.0 & $17.9-24.5$ \\
\hline Illawarra Private & 93 & 363 & 25.6 & 21.8 & $17.0-27.2$ \\
\hline \multicolumn{6}{|l|}{ South Eastern Sydney } \\
\hline Royal Hospital for Women & 501 & 1890 & 26.5 & 23.5 & $21.2-25.9$ \\
\hline St. George & 259 & 1269 & 20.4 & 20.5 & $17.7-23.6$ \\
\hline Sutherland & 96 & 456 & 21.1 & 21.5 & $16.7-27.0$ \\
\hline Hurstville Community & 149 & 494 & 30.2 & 23.2 & $19.2-27.5$ \\
\hline Kareena Private & 97 & 302 & 32.1 & 23.9 & $18.9-29.4$ \\
\hline St George Private & 233 & 684 & 34.1 & 26.6 & $23.0-30.3$ \\
\hline Prince of Wales Private & 279 & 846 & 33.0 & 23.5 & $20.5-26.5$ \\
\hline \multicolumn{6}{|l|}{ Northern Rivers } \\
\hline Grafton Base & 80 & 247 & 32.4 & 31.2 & $24.0-39.0$ \\
\hline Lismore Base & 128 & 573 & 22.3 & 23.6 & $19.1-28.7$ \\
\hline Murwillumbah & 55 & 243 & 22.6 & 22.4 & $16.0-30.0$ \\
\hline Tweed Heads & 70 & 372 & 18.8 & 22.7 & $16.7-29.6$ \\
\hline \multicolumn{6}{|l|}{ Mid North Coast } \\
\hline Coffs Harbour & 102 & 322 & 31.7 & 29.3 & $23.3-35.8$ \\
\hline Kempsey & 26 & 154 & 16.9 & 20.1 & $11.8-30.9$ \\
\hline Port Macquarie Base & 76 & 338 & 22.5 & 23.1 & $17.4-29.6$ \\
\hline Manning Base & 52 & 349 & 14.9 & 17.7 & $12.3-24.3$ \\
\hline New England & & & & & \\
\hline Armidale & 25 & 201 & 12.4 & 14.6 & $8.4-23.1$ \\
\hline Inverell District & 19 & 110 & 17.3 & 24.7 & $13.1-40.6$ \\
\hline Moree District & 22 & 122 & 18.0 & 24.5 & $13.7-38.9$ \\
\hline Tamworth Base & 78 & 327 & 23.9 & 22.2 & $16.8-28.3$ \\
\hline
\end{tabular}

Source: NSW Midwives Data Collection, Epidemiology and Surveillance Branch, NSW Department of Health.

\# Hospitals with more than 100 deliveries in the period 1 July-31 December 2000. 


\section{TABLE 118 (continued)}

CRUDE AND ADJUSTED CAESAREAN SECTION RATES BY HOSPITAL, NSW JULY-DECEMBER 2000\#

\begin{tabular}{|c|c|c|c|c|c|}
\hline $\begin{array}{l}\text { Health Area and } \\
\text { hospital }\end{array}$ & $\begin{array}{c}\text { Caesarean } \\
\text { sections } \\
\text { No. }\end{array}$ & $\begin{array}{c}\text { Births } \\
\text { No. }\end{array}$ & $\begin{array}{c}\text { Crude } \\
\text { rate } \\
\%\end{array}$ & $\begin{array}{c}\text { Adjusted } \\
\text { rate } \\
\%\end{array}$ & $\begin{array}{l}99 \% \text { confidence interval } \\
\text { of adjusted rate }\end{array}$ \\
\hline \multicolumn{6}{|l|}{ Macquarie } \\
\hline $\begin{array}{l}\text { Dubbo Base } \\
\text { Mudgee }\end{array}$ & $\begin{array}{r}105 \\
25\end{array}$ & $\begin{array}{l}608 \\
108\end{array}$ & $\begin{array}{l}17.3 \\
23.1\end{array}$ & $\begin{array}{l}21.8 \\
24.8\end{array}$ & $\begin{array}{l}17.0-27.2 \\
14.6-37.6\end{array}$ \\
\hline \multicolumn{6}{|l|}{ Mid Western } \\
\hline $\begin{array}{l}\text { Bathurst Base } \\
\text { Orange Base }\end{array}$ & $\begin{array}{l}91 \\
86\end{array}$ & $\begin{array}{l}278 \\
377\end{array}$ & $\begin{array}{l}32.7 \\
22.8\end{array}$ & $\begin{array}{l}31.1 \\
22.5\end{array}$ & $\begin{array}{l}24.4-38.4 \\
17.2-28.4\end{array}$ \\
\hline \multicolumn{6}{|l|}{ Far West } \\
\hline Broken Hill Base & 17 & 133 & 12.8 & 18.2 & $9.1-31.3$ \\
\hline Griffith Base & 51 & 234 & 21.8 & 22.9 & $16.0-31.0$ \\
\hline Wagga Wagga Base & 92 & 416 & 22.1 & 21.5 & $16.6-27.0$ \\
\hline Calvary, Wagga Wagga & 48 & 210 & 22.9 & 20.9 & $14.5-28.5$ \\
\hline \multicolumn{6}{|l|}{ Southern } \\
\hline Goulburn Base & 36 & 161 & 22.4 & 27.5 & $17.9-39.3$ \\
\hline Queanbeyan & 20 & 160 & 12.5 & 16.5 & $8.8-27.3$ \\
\hline Young & 23 & 107 & 21.5 & 23.1 & $13.2-35.9$ \\
\hline TOTAL NSW & 9434 & 43179 & 21.8 & 21.7 & $21.2-22.2$ \\
\hline
\end{tabular}

Source: NSW Midwives Data Collection, Epidemiology and Surveillance Branch, NSW Department of Health.

\# Hospitals with more than 100 deliveries in the period 1 July-31 December 2000. 


\section{APPENDIX 1}

\section{DESCRIPTION OF SELECTED BIRTH DEFECTS}

The following include descriptions of some of the birth defects included in this report :

Anencephaly

Absence of the cranial vault, with the brain tissue completely missing or markedly reduced.

Spina bifida

Defective closure of the bony encasement of the spinal cord, through which the spinal cord may protrude.

Encephalocele

Protrusion of brain through a congenital opening in the skull

Hydrocephalus

Dilatation of the cerebral ventricles accompanied by an accumulation of cerebral fluid within the skull.

Buphthalmos

Enlargement and distension of the fibrous coats of the eye.

Hypospadias

The opening of the urethra lies on the underside of the penis or on the perineum.

Epispadias

Absence of the upper wall of the urethra. The opening of the urethra lies on the dorsum of the penis in males, and anterior to or onto the clitoris in females.

Chordee

Downward bowing of the penis.

Talipes equinovarus

A deformity of the foot in which the heel is elevated and turned outward.

Polydactyly Presence of additional fingers or toes on hands or feet.

Syndactyly Attachment of adjacent fingers or toes on hands or feet

Craniosynostosis Premature closure of the sutures of the skull.

Exomphalos Herniation of the abdominal contents into the umbilical cord.

Gastroschisis

A defect in the abdominal wall not involving the umbilicus and through which the abdominal contents herniate.

Cystic hygroma

A sac, cyst or bursa distended with fluid.

\section{APPENDIX 2}

BIRTH DEFECT EXCLUSION LIST

The following is a general list of minor defects and non-structural disorders which are excluded from the NSW Birth Defects Register. For further details, please contact the NSW Birth Defects Register (see Further Information, p.17).

Abnormal palmar creases

Accessory nipples

Balanced chromosomal translocation (unless occurring with structural defects)

Birthmarks (single, $<4 \mathrm{cms}$ diameter)

Bronchopulmonary dysplasia

Cerebral palsy

Clicky hips

Congenital infections (unless occurring with structural defects)

Congenital neoplasms/tumours (exception: cystic hygroma)

Developmental disability

Deviated nasal septum

Fetal alcohol syndrome

Glucose-6-phosphate dehydrogenase (G6PD) deficiency

Haemophilia

Heart murmurs (functional)

Hernia (epigastric, hiatus, inguinal, umbilical)

Hydrocele (testis)

Hypoplastic lung (less than 37 weeks gestation)

Imperforate hymen

Inborn errors of metabolism other than phenylketonuria, galactosemia and congential hypothyroidism.
Intrauterine growth retardation

Low birthweight

Meconium ileus

Minor ear anomalies

Minor finger/hand anomalies

Minor toe/foot anomalies

Muscular dystrophies \& myopathies

Oesophageal reflux

Patent ductus arteriosus (less than 37 weeks gestation)

Pilonidal sinus

Sacral dimples

Single umbilical artery (unless occurring with structural defects)

Skin tag

Strabismus

Talipes (exception: those requiring surgery)

Tongue tie

Undescended testes (exception: those requiring surgery)

Webbing of 2 nd \& 3rd toes

Wide sutures 


\section{APPENDIX 3}

MATERNAL COUNTRIES OF BIRTH AND COUNTRY OF BIRTH GROUPS

\section{English speaking}

Australia

Christmas Island

Cocos (Keeling) Islands

Norfolk Island

New Zealand

United Kingdon

Channel Islands

Isle of Man

Ireland

Bermuda

Canada

United States of America

South Africa

\section{Central and South America}

Argentina

Bolivia

Brazil

Chile

Colombia

Ecuador

Falkland Islands

French Guiana

Guyana

Paraguay

Peru

Surinam

Uruguay

Venezuela

Belize

Costa Rica

El Salvador

Guatamala

Honduras

Mexico

Nicaragua

Panama

Antigua and Barbuda

Bahamas

Barbados

Cayman Islands

Cuba

Grenada

Guadeloupe

Jamaica

Netherlands Antilles

Puerto Rico

St Kitts-Nevis

St Lucia

St Vincent and the Grenadines

Trinidad and Tobago

Turks and Caicos Islands

Eastern Europe, Russia,

Central Asian and Baltic States

Bulgaria

Czechoslovakia

Hungary

Poland

Romania

Armenia

Azerbaijan

Belarus (formerly Byelorussia)

Estonia

Georgia

Kazakhstan

Kyrgyzstan (formerly Kirghizia)

Latvia

Lithuania

Moldova (formerly Moldavia)

Russian Federation

Ukraine

Uzbekistan
Melanesia, Micronesia and

\section{Polynesia}

New Caledonia

Papua New Guinea

Solomon Islands

Vanuatu

Guam

Kiribati

Nauru

Cook Islands

Fiji

French Polynesia (including

Tahiti)

Niue

American Samoa

Western Samoa

Tokelau

Tonga

Tuvalu

Wallis and Fortuna

Middle East and Africa

Bahrain

Gaza Strip

Iran

Iraq

Israel

Jordan

Kuwait

Lebanon

Qatar

Saudi Arabia

Syria

Turkey

United Arab Emirates

West Bank

Yemen

Algeria

Egypt

Libya

Mauritania

Morocco

Sudan

Tunisia

Cameroon

Central African Republic

Congo

Cote d'Ivoire

Gambia

Ghana

Guinea-Bissau

Liberia

Mali

Nigeria

Senegal

Sierra Leone

Zaire

Angola

Botswana

Djibouti

Ethiopia

Kenya

Malawi

Mauritius

Mozambique

Namibia

Reunion

Rwanda

Seychelles

Somalia

Swaziland

Tanzania

Uganda

Zambia

Zimbabwe

\section{North East Asia}

China (excluding Taiwan)

Hong Kong

Japan

North Korea

South Korea

Macau

Mongolia

Taiwan

\section{South East Asia}

Brunei

Cambodia

Indonesia

Laos

Malaysia

Burma (Myanmar)

Philippines

Singapore

Thailand

Vietnam

\section{Southern Asia}

Afganistan

Bangladesh

Bhutan

India

Maldives

Nepal

Pakistan

Sri Lanka

\section{Southern Europe}

Albania

Andorra

Cyprus

Gibraltar

Greece

Italy

Malta

Portugal

Spain

Former Yugoslavia (not

otherwise defined)

Croatia

Slovenia

\section{Western and Northern Europe}

Austria

Belgium

France

Germany (United)

Luxembourg

Netherlands

Switzerland

Denmark

Faeroe Islands

Finland

Iceland

Norway

Sweden 


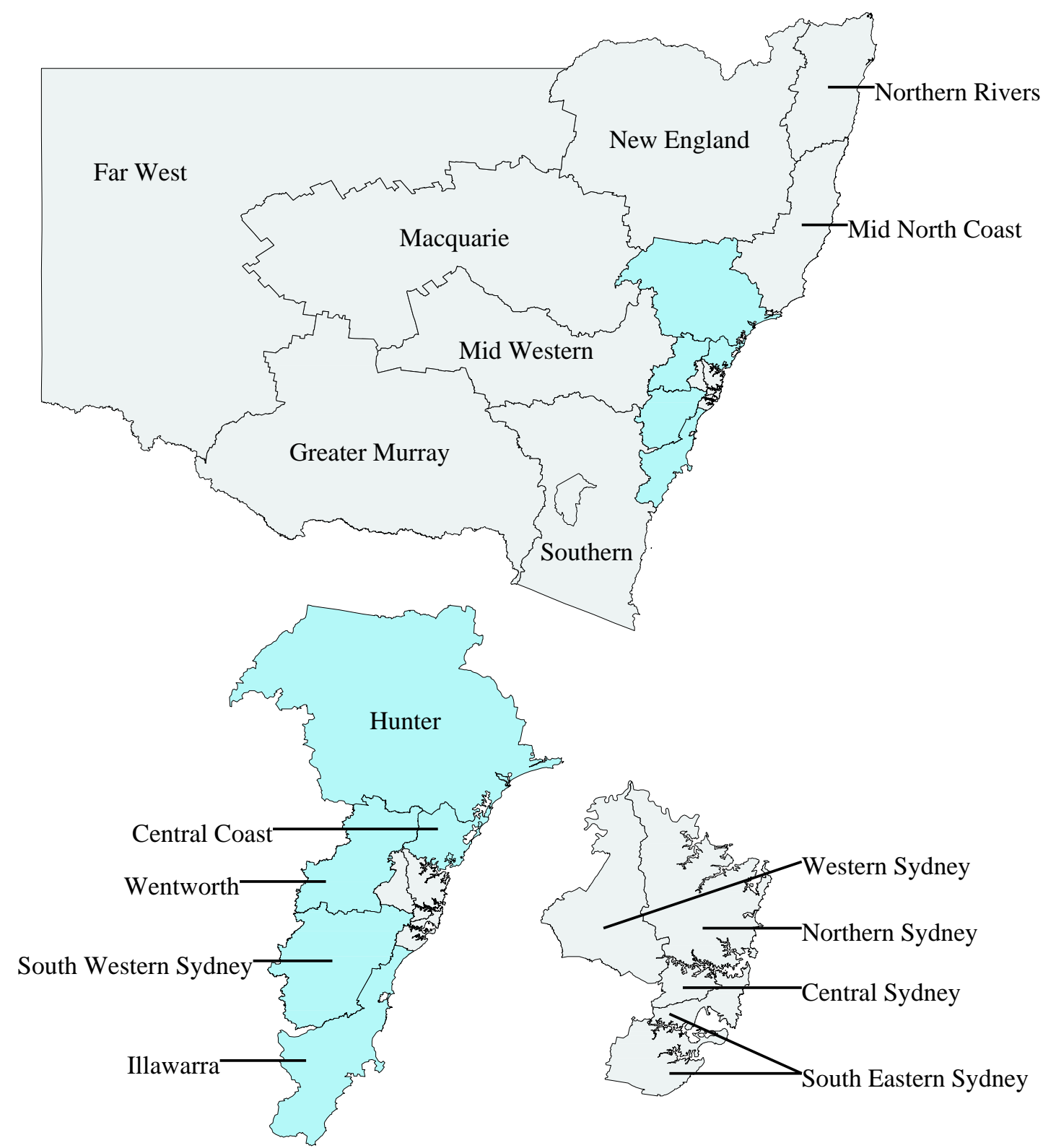

